\title{
Methods for Preferential Sampling in Geostatistics
}

\author{
by \\ Daniel Dinsdale
}

MMath, The University of York, 2013

A THESIS SUBMITTED IN PARTIAL FULFILLMENT OF

THE REQUIREMENTS FOR THE DEGREE OF

DOCTOR OF PHILOSOPHY

in

The Faculty of Graduate and Postdoctoral Studies

(Statistics)

THE UNIVERSITY OF BRITISH COLUMBIA

(Vancouver)

October 2018

(C) Daniel Dinsdale 2018 
The following individuals certify that they have read, and recommend to the Faculty of Graduate and Postdoctoral Studies for acceptance, the dissertation entitled:

\section{Methods for Preferential Sampling in Geostatistics}

submitted by Daniel Dinsdale in partial fulfillment of the requirements for the degree of Doctor of Philosophy in Statistics.

\section{Examining Committee:}

Matías Salibián-Barrera, Statistics

Supervisor

William J. Welch, Statistics

Supervisory Committee Member

Alexandre Bouchard-Côté, Statistics

University Examiner

Douw Steyn, Earth, Ocean and Atmospheric Sciences

University Examiner

\section{Additional Supervisory Committee Members:}

James V. Zidek, Statistics

Supervisory Committee Member 


\section{Abstract}

Preferential sampling in geostatistics occurs when the locations at which observations are made may depend on the spatial process that underlines the correlation structure of the measurements. If ignored, this may affect the parameter estimates of the model and the resulting spatial predictions. In this thesis we first show that previously proposed Monte Carlo estimates for the likelihood function may not be approximating the desired function. Furthermore, we argue that for preferential sampling of moderate complexity, alternative and widely available numerical methods to approximate the likelihood function produce better results than Monte Carlo methods. We illustrate our findings on various data sets, include the biomonitoring Galicia dataset analysed previously in the literature.

Research on preferential sampling has so far been restricted to stationary sampling locations such as monitoring sites. In this thesis we also expand the methodology for applicability in cases where the sensors are moving through the domain of interest. More specifically, we propose a flexible framework for inference on preferentially sampled fields, where the process that generates the sampling locations is stochastic and moving through a 2-dimensional space. The main application of these methods is the sampling of ocean temperature fields by marine mammal mounted sensors. This is an area of research which has grown drastically over the past 25 years and is providing scientists with a wealth of new oceanographic information in areas of our oceans previously not well understood. We show that standard geostatistical models may not be reliable for this type of data, due to the possibility that the regions visited by the animals may depend on the ocean temperatures, hence resulting in a type of preferential sampling. Our simulation studies confirm that predictions obtained from the preferential sampling model are more reliable when this phenomenon is present, and they compare very well to the standard ones when there is no preferential sampling. We apply our methods to sea surface temperature data collected by Southern elephant seals in the Southern Indian ocean and show how predictions of sea surface temperature fields using this data may vary when accounting for the preferential movement. 


\section{Lay Summary}

This research provides new methods to correct for the issue of preferential sampling. This is the phenomenon where the locations at which we observe some geographic measure, such as temperature or pollutant levels, have been chosen in a way that may depend on the process we are measuring. For example, measuring pollutant levels in Canada only near cities may mislead our understanding of pollutant levels across the country in general, since our measures will be larger than those obtained by randomly placing monitoring sites across the country.

We extend research surrounding preferential sampling from stationary monitoring sites to the collection of oceanographic measurements, such as water temperature, collected by tags attached to marine mammals. This data can be used to help us understand changing ocean dynamics in sparsely sampled areas, such as the Antarctica. However, movement of these animals might be influenced by these temperatures resulting in misleading analyses if ignored. 


\section{Preface}

This thesis is the original work of the author Daniel Dinsdale under the supervision of Prof. Matías Salibián-Barrera.

A version of Chapters 445 has been published [Dinsdale DR, SalibianBarrera M. Methods for preferential sampling in geostatistics. Journal of the Royal Statistical Society Series C. 2018. https://doi.org/10.1111/ rsSC.12286] and a version of Chapter 8 has been submitted for peer review [Dinsdale DR, Salibian-Barrera M. Modelling ocean temperatures from bioprobes under preferential sampling]. The ideas for both these works have

been jointly developed by Daniel Dinsdale and Prof. Matías Salibián-Barrera with the majority of computational work and manuscript writing conducted by Daniel Dinsdale. 


\section{Table of Contents}

Abstract $\ldots \ldots \ldots \ldots \ldots \ldots \ldots \ldots$ iii

Lay Summary $\ldots \ldots \ldots \ldots \ldots \ldots \ldots \ldots$ iv

Preface $\ldots \ldots \ldots \ldots \ldots \ldots \ldots \ldots \ldots$

Table of Contents . . . . . . . . . . . . . . . . vi vi

List of Tables $\ldots \ldots \ldots \ldots \ldots \ldots \ldots \ldots \ldots$

List of Figures . . . . . . . . . . . . . $\mathrm{xi}$

Glossary . . . . . . . . . . . . . . . . . . . Xviii

Notation $\ldots \ldots \ldots \ldots \ldots \ldots \ldots \ldots \ldots \ldots \ldots$

Acknowledgements ................. xxii

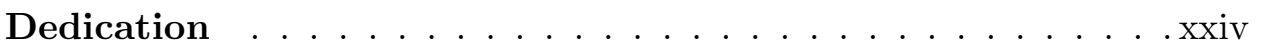

1 Introduction $\ldots \ldots \ldots \ldots \ldots \ldots \ldots \ldots \ldots \ldots$

1.1 Thesis Outline . . . . . . . . . . . . . 3

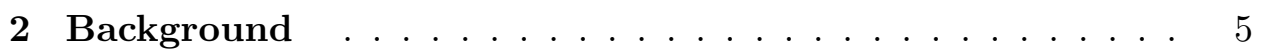

2.1 Geostatistics . . . . . . . . . . . . . . . 5

2.2 Stochastic Processes . . . . . . . . . . . . . . 6

2.2.1 Spatial Gaussian Processes and Fields . . . . . . . 6 6

2.2 .2 Stationarity . . . . . . . . . . . . 7

2.2.3 Continuity and Differentiability $\ldots \ldots \ldots \ldots$

2.2.4 Covariance Families . . . . . . . . . . . . . . 8

2.3 Maximum Likelihood Estimation and Spatial Prediction . . . 9

2.3.1 Maximum Likelihood Estimation _. . . . . . . . . 9 9

2.3.2 Spatial Prediction . . . . . . . . . . . . . 12 
2.4 Spatial Point Processes . . . . . . . . . . . . . 14

2.5 Stochastic Differential Equations . . . . . . . . . . 15

3 Preferential Sampling . . . . . . . . . . . . . . . . . . 18

3.1 Preferential Sampling Overview . . . . . . . . . . 18

3.2 Sampling from Point Processes . . . . . . . . . . . . . 21 21

3.2.1 Example of Preferential Point Processes . . . . . . . . 22

3.2.2 Drawbacks of the Point Process Approach . . . . . . 26

3.3 Movement Processes . . . . . . . . . . . . . . . . 27

4 Maximum Likelihood Estimation Under Preferential Sam-

pling . . . . . . . . . . . . . . . . . . . . . . . . . 29

4.1 Specifying the Preferential Likelihood . . . . . . . . . . 29

4.2 Monte Carlo Approximation to the Preferential Likelihood . 30

4.3 Monte Carlo Solution Problems and Correction . . . . . . . . 32

4.3.1 Implicit Conditioning of $\mathbf{X} \ldots \ldots \ldots . . \ldots 32$

4.3.2 Specification of $[\mathbf{S} \mid \mathbf{X}, \mathbf{Y}] \quad \ldots \ldots \ldots \ldots$

4.3.3 An Alternative Monte Carlo Solution . . . . . . . . 34

4.3.4 Other Modelling Approaches . . . . . . . . . . 35

4.4 A Laplace Approximation to the Likelihood . . . . . . . . . 36

4.4.1 The Laplace Approximation . . . . . . . . . . 36

4.4.2 Automatic Differentiation . . . . . . . . . . . . 38

4.4.3 Implementation in Template Model Builder . . . . . . 42

4.5 Simulation Comparison . . . . . . . . . . . . 44

4.5.1 Data Description . . . . . . . . . . . . 44

4.5.2 Parameter Estimation . . . . . . . . . . . . . 44 4

4.6 Application to the Galicia Moss Data . . . . . . . . . . . 48

5 Spatial Prediction Under Preferential Sampling . . . . . 51

5.1 Naive Prediction . . . . . . . . . . . . . . . . . . 51

5.2 Preferential Predictive Distribution . . . . . . . . 53

5.3 Simulation Comparison . . . . . . . . . . . . 54 54

5.4 Comparison Between Predictive Distributions . . . . . . 56

6 Preferential Sampling Analysis _ . . . . . . . . . 6 60

6.1 Ozone Data Analysis . . . . . . . . . . . . . . . . 60

6.2 Comparison Between Various Degrees of Preferential Sam-

pling ..................... 66 
7 Modelling Marine Mammal Movement . . . . . . . . . . . 71

7.1 Background on Marine Tagging . . . . . . . . . . 71

7.2 Modelling of Marine Mammal Movement . . . . . . . . . . 72

7.2.1 State Space Movement Models . . . . . . . . . . . 75

7.2.2 Correlated Random Walk Models . . . . . . . . . 78

7.2.3 Modelling Movement Using Template Model Builder

$(\mathrm{TMB}) \ldots \ldots \ldots \ldots$

8 Preferential Sampling of Ocean Temperatures . . . . . . 87

8.1 Marine Bio-logging . . . . . . . . . . . . 87

8.2 Preferential Sampling of Ocean Temperatures . . . . . . 88

8.2.1 Data Description . . . . . . . . . . . 90

8.2 .2 Assumptions . . . . . . . . . . . . . . . 92

8.3 A Preferential Model for Marine Mammal Movement . . . . 92

8.3.1 A Preferential Correlated Random Walk Model ... . 93

8.3.2 Evaluating the Preferential Correlated Random Walk

Model . . . . . . . . . . . . . . . . 95

8.3.3 Advantage in Comparison to Point Process Model . . 97

8.4 Simulation Experiments . . . . . . . . . . . . . . . . . 98

8.4 .1 Simulation $1 \ldots \ldots \ldots$

8.4 .2 Simulation $2 \ldots \ldots \ldots$

8.5 Real Data Example . . . . . . . . . . . . . . . . . 112

9 Preliminary Extensions . . . . . . . . . . . . . . . . . . . 121

9.1 Extending Ocean Temperatures to New Depths . . . . . . . 121

9.2 Non-Constant Mean Trend . . . . . . . . . . . . . . . . . . . 125

9.2.1 Comparison Between Constant and Linear Trend Preferential Performance . . . . . . . . . . . . . 128

9.2.2 Comparison Between Preferential and Non-Preferential

Trend Performance . . . . . . . . . . . 129

9.2.3 Concluding Thoughts . . . . . . . . . . . . . 132

10 Concluding Remarks and Future Work . . . . . . . . . . 134

10.1 Future Work . . . . . . . . . . . . . . . . . 136

10.1.1 Including Location Uncertainty . . . . . . . . . 136

10.1.2 Combining with Deterministic Model Outputs . . . . 137

10.1.3 Introducing Time Varying Latent Fields . . . . . . . 138

10.1.4 Improving Computational Efficiency . . . . . . . . . 139

Bibliography . . . . . . . . . . . . . . . . . . . . . 142 


\section{Appendices}

A Efficient Evaluation of High Dimensional Gaussian Fields 156

A.1 Stochastic Partial Differential Equation Approximation . . . 156

A.2 Implementation of SPDE's in TMB . . . . . . . . . . . . 158

B Extended Kalman Filter . . . . . . . . . . . . . . . 163

C Template Model Builder Example Code . . . . . . . . . . 164

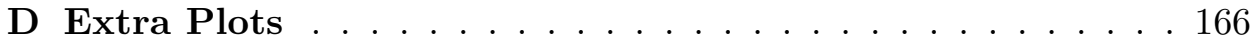




\section{List of Tables}

$3.195 \%$ Confidence intervals for prediction error $(\mathrm{PE})$ and bias at prediction location $\mathrm{x}_{0}=(0.06,0.06) \ldots \ldots \ldots$

4.1 Parameter estimates, standard errors and correlations for the joint 1997 and 2000 data model using TMB. . . . . . . . 49

5.1 Comparison of approximate 95\% confidence intervals for the prediction errors (PE) and bias between standard MLE and TMB over 500 independent simulations for preferential $(\beta=$ 1.5) and non-preferential $(\beta=0)$ models. . . . . . . . 55 


\section{List of Figures}

2.1 Left Panel: Example of the potential function $H(\mathbf{X}(t), \boldsymbol{\theta})=$ $X_{1}(t)^{2}+X_{2}(t)^{2}$. Right Panel: The respective drift function $\boldsymbol{\mu}(\mathbf{X}(t), \boldsymbol{\theta})=-2\left(X_{1}(t), X_{2}(t)\right)^{\top} \ldots \ldots \ldots \ldots$

3.1 Example of sampling locations using the inhomogenous point process with intensity (3.4) with varying $\beta$ values on the same field $\mathbf{S}$. The $\bar{y}$ value is sample mean for that data set. . . . . 24

3.2 Parameter estimates over 500 simulated data sets with varying $\beta$ parameters. True parameter values are marked as black horizontal lines. . . . . . . . . . . . . . . .

4.1 Directed graph of the function in $(4.22)$.

4.2 Example of a single realisation of $S$ and corresponding 100 sampling locations generated using the preferential $(\beta=1.5)$ and non-preferential $(\beta=0)$ approach described in Section 4.5.1. 45

4.3 Parameter estimates over 500 preferentially simulated data sets with true parameter values marked as black lines. The abbreviations DMS 10 and NonPref stand for the Monte Carlo method of Diggle et al. (2010) and standard MLE (non-preferential) estimation respectively. . . . . . . . . . . . . . 46

4.4 Parameter estimates over 500 non-preferentially simulated data sets with true parameter values marked as black lines. The abbreviations DMS10 and NonPref stand for the Monte Carlo method of Diggle et al. (2010) and standard MLE (nonpreferential) estimation respectively. . . . . . . . . . .

4.5 Survey sampling locations for 1997 and 2000 lead concentration data sets. Map tiles by Stamen Design, under CC BY 3.0. Data by OpenStreetMap, under ODbL. . . . . . . . . 49

5.1 Comparison between mean ignorance scores across all 500 data sets in the preferentially sampled case (left panel) and non-preferentially sampled (right panel). . . . . . . . 5 57 
5.2 Comparison of root-mean-square prediction error and mean ignorance scores using corrected parameter estimates and different formulations for $[\mathbf{S} \mid \mathbf{Y}, \mathbf{X}] \ldots \ldots \ldots \ldots \ldots$

5.3 Comparison between true field (a) and both preferential (b) and non-preferential (c) predictions. Note that both prediction methods use the same corrected parameter estimates from TMB, but the preferential model uses the mode of $[\mathbf{S} \mid \mathbf{Y}, \mathbf{X}]$ and the non-preferential model uses kriging. . . . . . . . . .

6.1 Example data set from the Ozone analysis. Black circles are monitoring sites in the example data set and red triangles reserved for prediction. Map tiles by Stamen Design, under CC BY 3.0. Data by OpenStreetMap, under ODbL. . . . . . 61

6.2 Comparison of ozone prediction on the example data set between kriging using standard MLE plug-in parameters and the mode of $[\mathbf{S} \mid \mathbf{X}, \mathbf{Y}]$ obtained from TMB. Circles are the sampling locations and triangles the test sites with the size of each triangle the relative root-mean-square-prediction-error. . . . . 63

6.3 Comparison between scoring functions of kriging prediction using standard MLE plug-in parameters and the mode of $[\mathbf{S} \mid \mathbf{X}, \mathbf{Y}]$ obtained from TMB across 30 prediction locations. Smaller root-mean-square prediction errors and mean ignorance scores are considered better. . . . . . . . . . . 65

6.4 Comparison between bias of the mean parameter estimate $\hat{\mu}$ using Laplace approximation in TMB and a non-preferential likelihood. . . . . . . . . . . . . .

6.5 Comparison between prediction bias using mode of $[\mathbf{S} \mid \mathbf{Y}, \mathbf{X}]$ and ordinary kriging with plug-in parameters obtained from a non-preferential likelihood. . . . . . . . . . . . 68

6.6 Comparison between mean ignornace score differences using mode of $[\mathbf{S} \mid \mathbf{Y}, \mathbf{X}]$ and ordinary kriging with plug-in parameters obtained from a non-preferential likelihood. . . . . . . .

7.1 Left Panel: Example of 6 simulated tracks each consisting of 150 observations using an Ornstein-Uhlenbec process, with arrow direction and tail length showing direction and relative strength of drift function (expected movement) at that location. Right Panel: Potential function for the drift function used to generate the data in the left panel. . . . . . . . . 74 
7.2 Example of 3 simulated tracks each consisting of 150 observations using first-difference correlated random walk from (7.6). All tracks are initalised with the first two observations of $(0,0)$ and $(1,-5)$ with the purple, green and red tracks corresponding to $\gamma=0,0.5$ and 0.8 respectively. . . . . . . . . 80

7.3 Example of 3 simulated tracks each consisting of 100 observations using the continuous-time correlated random walk model from (7.8)-(7.9). The purple, green and red tracks correspond to $\alpha=0.2,0.5$ and 1 respectively. The black diagonal line shows the mean velocity direction $(\boldsymbol{\beta})$, which is heading in a North-Easterly direction. . . . . . . . . . . .

7.4 Panels (a)-(b) True latent longitudinal and latitudinal velocities for the $\alpha=0.2$ (purple) track between times 25-75 given in red. The black line and shaded region correspond to state estimates and 95\% confidence region. Panel (c) Section of the track between times 25-75 corresponding to the latent states.

7.5 Left Panel: Simulated data consisting of 300 sampling location observations taken at equidistant intervals, where pink lines show movement in the travelling state $(\gamma(t) \geq 0.7)$ and blue lines in the foraging state $(\gamma(t)<0.7)$ following AugerMéthé et al. (2017). Right Panel: True latent behavioural state $\gamma(t)$ used to generate the track as shown by the red line. The black line and shaded region are the $\hat{\gamma}$ estimates and $95 \%$ confidence intervals obtained using TMB. . . . . 86

8.1 Plot of all 66,519 sea surface temperature (SST) observations from Southern elephant seal platforms taken between 2nd February 2008 and 10th March 2013. SST's were approximated as the temperature at the maximum depth of 6 meters. Map tiles by Stamen Design, under CC BY 3.0. Data by OpenStreetMap, under ODbL. . . . . . . . . . . . 91

8.2 Example of a simulated data set of 3 tracks generated using the field and movement parameters defined in simulation 1 , resulting in a moderate preferential sampling effect. . . . . . 999

8.3 Field parameter estimates over 50 preferentially simulated data sets with true parameter values marked as black lines. The abbreviations NonPref and Pref stand for the standard MLE (non-preferential) estimation and those using the preferential PCRW model of Section 8.3 respectively. . . . . . 101 
8.4 Movement parameter estimates over 50 preferentially simulated data sets with true parameter values marked as black lines. . . . . . . . . . . . . . . . . . . . . 102

8.5 Field parameter estimates over 50 non-preferentially simulated data sets with true parameter values marked as black lines. The abbreviations NonPref and Pref stand for the standard MLE (non-preferential) estimation and those using the PCRW model of Section 8.3 respectively. . . . . . . . . . 103

8.6 Comparison of root-mean-square prediction error (RMSPE) difference, mean ignorance score (MIGN) difference and location ignorance score (LIGN) difference respectively across 50 preferentially simulated data sets. . . . . . . . . . . . 106

8.7 Comparison of root-mean-square prediction error (RMSPE) difference, mean ignorance score (MIGN) difference and location ignorance score (LIGN) difference respectively across 50 non-preferentially simulated data sets. . . . . . . . . . . 107

8.8 Example of a simulated data set of 3 tracks generated using the field and movement parameters defined in simulation 2, resulting in a small preferential sampling effect. . . . . . . . . 108

8.9 Field parameter estimates over 50 data sets with true parameter values marked as black lines for simulation 2 . The abbreviations NonPref and Pref stand for the standard MLE (nonpreferential) estimation and those using the PCRW model of Section 8.3 respectively. . . . . . . . . . . . . . . . . 110

8.10 Comparison of root-mean-square prediction error (RMSPE) difference, mean ignorance score (MIGN) difference and raw values of the location ignorance score (LIGN) difference respectively across 50 data sets from simulation 2 . . . . . . . 111

8.11 Plot all 3369 observations from the subset of Southern elephant seal data analysed in Section 8.5. The first observation was taken on July 1st 2012 and the final observation on 30th September 2012. Panel (a) shows raw latitude and longitude plots with corresponding temperatures whilst Panel (b) shows the corresponding transformed locations using a scaled version of the Universal Transverse Mercator (UTM) projection (zone 43). Map tiles by Stamen Design, under CC BY 3.0. Data by OpenStreetMap, under ODbL. . . . . . . . . . . 114

8.12 Comparison between preferential and standard MLEs for the field parameters over 50 data sets consisting of a sub-sample of 13 tracks with 30 observations each. . . . . . . . . . . 115 
8.13 Movement parameter estimates using the preferential model over 50 data sets consisting of a sub-sample of 13 tracks with 30 observations each. . . . . . . . . . . . . . 116

8.14 Panel (a): Monthly average field $\left({ }^{\circ} \mathrm{C}\right)$ obtained via the Simple Ocean Data Assimilation ocean/sea ice reanalysis version 3 (SODA3) analysis (Carton et al., 2018). Panel (b): Median of prediction difference between preferential and nonpreferential models $\left({ }^{\circ} \mathrm{C}\right)$. Positive values imply that the preferential model tends to increases temperature prediction at that location and negative the opposite. Panels (c)-(d): Comparison of predicted fields using preferential and non-preferential models $\left({ }^{\circ} \mathrm{C}\right) . \ldots \ldots \ldots \ldots$. . . . . . . . . . . . . . 118

8.15 Comparison of root-mean-square prediction error (RMSPE) difference, mean ignorance score (MIGN) difference and location ignorance score (LIGN) difference respectively across 50 simulated data sets between preferential and non-preferential temperature predictions. . . . . . . . . . . . . . . 119

9.1 Plot all 2264 observations from the underwater data set taken at 50 meters below sea level. The first observation was taken on July 1st 2012 and the final observation on 30th September 2012. Panel (a) shows raw latitude and longitude plots with corresponding temperatures whilst Panel (b) shows the corresponding transformed locations using a scaled version of the Universal Transverse Mercator (UTM) projection (zone 43). Map tiles by Stamen Design, under CC BY 3.0. Data by OpenStreetMap, under ODbL. . . . . . . . . . . . . . 123

9.2 Panels (a)-(c): Parameter estimate comparisons for $\mu, \phi$ and $\sigma^{2}$ using the non-preferential and preferential model over 50 data sets consisting of a sub-sample of 9 tracks of 30 observations each. Panel (d): Parameter estimates for the preferential parameter $\alpha$ in the preferential model. . . . . . . . 124 
9.3 Panel (a): Simple Ocean Data Assimilation ocean/sea ice version 3 (SODA3) reanalysis (Carton et al., 2018) model output $\left({ }^{\circ} \mathrm{C}\right)$. Panel (b): The mean preferential model prediction $\left({ }^{\circ} \mathrm{C}\right)$. Panel (c): Median of prediction difference between preferential and non-preferential models $\left({ }^{\circ} \mathrm{C}\right)$. Positive values imply that the preferential model tends to increases temperature prediction at that location and negative the opposite. Panels (d)-(f): Comparison of root-mean-square prediction error (RMSPE) difference, mean ignorance score (MIGN) difference and location ignorance score (LIGN) difference respectively across 50 simulated data sets. . . . . . . . . . . 126

9.4 Panels (a)-(c) Comparison between SODA3 data set model output with average preferential predictions (linear and constant mean trend) over 50 subsamples of the seas surface temperature data set described in Section $8.5\left({ }^{\circ} \mathrm{C}\right)$. Panel (d): The median difference between the two preferential models $\left({ }^{\circ} \mathrm{C}\right)$ with negative values of this difference meaning lower predicted values using the linear trend model and positive values greater predictions using this model in comparison to the constant trend equivalent. Panel (e): Comparison between $\hat{\mu}_{2}$ and $\hat{\mu}_{3}$ from $(9.1) \ldots \ldots \ldots \ldots$. . . . . . . . . . . . .

9.5 Comparison of predictive performance compared to SODA3 model output between preferential models with constant and linear trends using scoring functions from $(9.3) \ldots . . . . .131$

9.6 Comparison of predictive performance compared to SODA3 model output between preferential and non-preferential models with linear trends using scoring functions from $(9.5) \quad \ldots 133$

A.1 Comparison of various spatial meshes using inla.mesh.create function in R-INLA. Each label is the type of refinement used in the specification of the mesh. . . . . . . . . . . 160

A.2 Example of lattice and sampling location combined mesh. Here the red points are sampling locations and blue points the lattice points used to approximate the integral of the intensity function and for prediction comparison. . . . . . . 161

D.1 Comparison between preferential and standard MLEs for the field parameters over 50 data sets described in Section 9.1 consisting of a sub-sample of 9 tracks of 30 observations each. 167 


\section{List of Figures}

D.2 Movement parameter estimates using the preferential model over 50 data sets described in Section 9.1 consisting of a subsample of 9 tracks of 30 observations each. . . . . . . . 168 


\section{Glossary}

Please refer to this list of acronyms used throughout the thesis. All acronyms are defined during their initial usage in the text.

$\begin{array}{ll}\text { AD } & \text { Automatic Differentiation } \\ \text { CRDT } & \text { Constrained Refined Delaunay Triangulation } \\ \text { CRW } & \text { Correlated Random Walk } \\ \text { CTCRW } & \text { Continuous Time Correlated Random Walk } \\ \text { CTD } & \text { Conductivity-Temperature-Depth tag } \\ \text { DCRW } & \text { Discrete time Correlated Random Walk } \\ \text { EKF } & \text { Extended Kalman Filter } \\ \text { GF } & \text { Gaussian Field } \\ \text { GFDL } & \text { Geophysical Fluid Dynamics Laboratory } \\ \text { GHRSST } & \text { Group for High Resolution Sea Surface Temperature } \\ \text { GMRF } & \text { Gaussian Markov Random Field } \\ \text { HMM } & \text { Hierarchical Movement Model } \\ \text { IGN } & \text { Ignorance score } \\ \text { INLA } & \text { Integrated Nested Laplace Approximations } \\ \text { KF } & \text { Kalman Filter } \\ \text { LHS } & \text { Left Hand Side } \\ \text { LIGN } & \text { Location Ignorance score } \\ \text { MC } & \text { Monte Carlo }\end{array}$




\section{Glossary}

MCMC Markov Chain Monte Carlo

MEOP Marine Mammals Exploring the Oceans Pole to Pole

MIGN Mean Ignorance score

MLE Maximum Likelihood Estimation

MUR Multi-scale Ultra-high Resolution (MUR) data set

NOAA National Oceanic and Atmospheric Administration

OU Ornstein-Uhlenbec

PCRW Preferential Correlated Random Walk

RHS Right Hand Side

RMSPE Root-Mean-Square-Prediction-Error

SDE Stochastic Differential Equation

SEaOS Southern Elephant Seals as Oceanographic Samplers

SODA Simple Ocean Data Assimilation ocean/sea ice reanalysis

SODA3 Simple Ocean Data Assimilation ocean/sea ice reanalysis version 3

SPDE Stochastic Partial Differential Equation

SSM $\quad$ State Space Model

SST Sea Surface Temperature

STE Stratosphere-Troposphere Exchange of ozone

UTM Universal Transverse Mercator coordinate system

TMB Template Model Builder

WOD World Ocean Database 


\section{Notation}

We define some common notation properties maintained throughout this thesis. Chapter specific notation are omitted here and defined when required.

Capitalised English alphabetic characters are used to denote random variables with their observed values denoted in lower case. Boldface characters are used to define vectors and matrices with scalars in non-bold face. For example $\mathbf{A}=\left(A_{1}, \ldots, A_{n}\right)^{\top}$ denotes an $n$-dimensional random variable and $\mathbf{a}=\left(a_{1}, \ldots, a_{n}\right)^{\top}$ a corresponding observed value. Commonly used examples in this thesis include:

$S \quad$ The continuous latent random field.

S A discretised version of the latent random field.

$S(\mathbf{u}) \quad$ The value of the continuous latent field at some location $\mathbf{u}$.

$\mathbf{S}(\mathbf{u}) \quad$ The value of the discretised latent field at some location $\mathbf{u}$.

$\mathbf{X} \quad$ An $n \times 2$ matrix of corresponding sampling locations.

Y An $n$-dimensional vector of response variables.

In general we will denote by $n$ the number of observations in the data set and $N$ the size of our discretised field vector $\mathbf{S}$.

We use Greek alphabetic characters to denote parameters, where again boldface characters are used to define vectors and matrices with scalars in non-bold face. Commonly used parameters are:

$\mu \quad$ Parameter for the constant mean of a latent field $S$.

$\phi \quad$ Parameter for the scale (range) of the Matérn covariance function.

$\sigma^{2} \quad$ Parameter for marginal variance of a latent field $S$.

$\tau^{2} \quad$ Parameter for the measurement error variance (partial sill). 


\section{Notation}

$\boldsymbol{\theta} \quad$ Full parameter vector consisting of all field and sampling process parameters.

Finally we include some other common notations:

$[W] \quad$ The density or probability mass function of the random object $W$.

$\|\cdot\| \quad$ The Euclidean distance.

$\mathcal{D} \quad$ The spatial domain of interest to the model.

$\mathbb{E}_{W} \quad$ The expectation with respect to a random object $W$. The subscript is omitted if only 1 random variable is involved.

$I_{n} \quad$ The $n \times n$ identity matrix consisting of 1's on the diagonal and 0 's everywhere else.

$N_{i}(\mathbf{A}, \mathbf{B})$ The $i$-dimensional multivariate normal distribution for some mean vector $\mathbf{A}$ and covariance matrix $\mathbf{B}$. When $i$ we have the univariate normal distribution and the subscript 1 is ignored.

$\nabla_{\mathbf{A}} \quad$ The gradient of a scalar function with respect to some vector $\mathbf{A}$.

$\boldsymbol{\mu}(\cdot) \quad$ The drift function (expected movement velocity) in a stochastic differential equation.

$\mathbb{R} \quad$ The 1-dimensional real number line.

$\mathbb{R}^{2} \quad$ The 2-dimensional real number space $\mathbb{R} \times \mathbb{R}$. 


\section{Acknowledgements}

I have been incredibly lucky to be surrounded, both in Canada and at home, by people who have offered me incredible amounts of support. Without them, I could never have embarked on my adventure to Vancouver and written this thesis.

First and foremost I would like to thank my supervisor Dr. Matías Salibián-Barrera for his continued support throughout my studies. Our discussions and his careful explanations have been extremely beneficial to my development during my time at UBC. Furthermore, on a personal level I am very thankful to have worked with someone of such a welcoming nature. Working together for 5 years has been incredibly enjoyable, especially thanks to our discussions on football (even when I am lamenting my choice of teams to support). I could not have asked for a better supervisor.

Thanks to Dr. Jim Zidek for his valuable discussions, particularly on the topic of preferential sampling, which were extremely valuable to my work. I also appreciate the valuable support of Dr. Nancy Heckman and Dr. Will Welch for their input on my work and to all the other professors in the department who helped me throughout the years.

When I first arrived at UBC with nothing to my name except what I could drag with me on my travels throughout the USA, I was immediately made to feel welcome by Andrea Sollberger. She has been a constant source of help and friendship to me and I will dearly miss our chats in the office (or maybe just the free chocolate). I will always fondly remember Andrea turning our department into long distance runners for a semester, we were never so fit! I would also like to thank Ali Hauschildt and Peggy Ng for their help, both of whom have been extremely kind in supporting me throughout my studies.

I would never have even made it to UBC without my friends and family back home. I will be eternally grateful to my parents, Joy and Barry, and sister Lucy for being so supportive of my move to Vancouver. Although I am sure they would have preferred me to be closer to home, they have been a constant source of support and I have been so thrilled to have them visit. I am incredibly lucky to have such a supporting family and I will be forever 
grateful. A lack of Easter chocolate has been their only lapse.

I have been so fortunate to meet many wonderful people in my time at UBC. Foremost I would like to thank Camila Casquilho, who has never failed to support me or pick me up during the hard times. Without her, my time in Canada would never have been so enjoyable. I also want to thank all the friends I have made at UBC who have supported me. When I first arrived I was very fortunate to meet many wonderful people who made me feel welcome right away. Destroying my insides with cheap Chinese food or using coupons for cheaper burgers alongside Andres Sanchez, Neil Spencer and Sean Jewell was inadvisable but highly enjoyable. Dinners hosted by Vincenzo Coia were some of my culinary highlights, in particular thanksgiving in my first term at UBC! Thanks to Chiara Di Gravio, Jack $\mathrm{Ni}$ and others for company on some fantastic trips, such as camping and Seattle visits in my first couple of years. Also thanks to Seong-Hwan Jun and Tingting $\mathrm{Yu}$ for great board-game evenings and chats.

Throughout my years as I have transitioned from eager first year to a battle-hardened veteran, I have met many more wonderful people. I always enjoyed football chats and matches with Jeff Bone, along with FIFA sessions with Derek Cho and Jonathan Agyeman. I never thought I would meet a Cricket fan from Ghana who appreciates James Vince, but Jonathan proved this assumption incorrect! Thanks to Elena Schurenkova for dragging me into the office when I had been hiding away with my sums for too long and to Joe Watson for the INLA chats that broadened my mind to areas that once seemed too overwhelming. It has also been a joy to embrace others cultures and share my own, or lack of. Credit to Creagh Briercliffe for trying to learn Geordie insults and Alexi Rodríguez-Arelis for his fish and chips discoveries! Thanks to numerous others who I have been so priviledged to meet such as David Kepplinger, Eric Fu, Qiong Zhang and Tanja Högg among many others.

Finally, thanks to everyone who played on the Mean Squared Terrors soccer (football) team. This small band of ragtag statisticians managed to progress from propping up the UBC departmental division to eventual winners after 4 years. I have never been so proud of a team since Sunderland broke the record for least number of points in a Premier League season. In particular, I greatly appreciate the dedication of long time members Camila Casquilho, Chiara Di Gravio, David Kepplinger, Derek Cho, Guohai Zhou, Jeff Bone, Jonathan Agyeman and Seong-Hwan Jun. 


\section{Dedication}

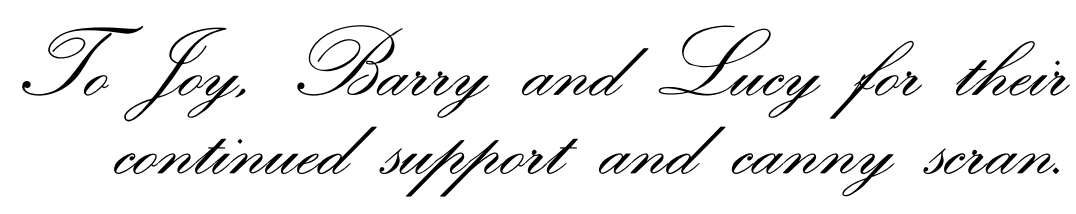




\section{Chapter 1}

\section{Introduction}

Geostatistics is an area of spatial statistics concerned with data consisting of finite samples of a spatially continuous phenomenon, with the aim of describing spatial patterns and performing prediction at unobserved locations. Born out of the need for miners to understand the distribution of ore grades and thickness, Matheron (1963) formally introduced the notion of a variable taking a definite value at every point in some 2-dimensional space, related through a spatial correlation. Examples of these variables include ore grades in a deposit and atmospheric measures such as temperature, pressure and pollutant levels.

Core to most geostatistical models is the assumption that sampling locations are independent of the phenomenon being measured. Take for example, the measuring of pollutant levels across the United Kingdom from stationary monitoring sites. In this case, standard geostatistical methods to infer properties of the pollutant field assume that the site locations provide no information on the process being measured and therefore our understanding of this phenomenon is not affected by considering the sampling locations non-random in the subsequent analysis. However, this might not always be the case. In this instance, as discussed by Zidek et al. (2014), the air pollution monitoring sites may be located in areas which the designer believed pollutant levels to be highest. This could be due to a multitude of reasons including practicality of recording important measures near areas of high population density (Gelfand et al., 2012), or even pursuit of political leverage. If this is the case, neglecting the impact of the site location bias in the subsequent analysis may result in inaccurate predictions which may be used for other purposes such as detecting noncompliance with air quality standards or to inform epidemiological studies estimating risks to health.

Preferential sampling refers to this situation, namely where the process that determines the data locations and the spatial field of interest may depend on each other (Diggle et al., 2010). If ignored, the effect of preferential sampling on subsequent inference can be two-fold. On the one hand, data collected in this way may tend to include a reduced range of the response variable. For example, if air pollution sites are located in higher pollutant 
regions, their measurements will tend to not include observations from lower pollutant regions. On the other hand, the locations at which data are collected might carry useful information about the variable of interest. In the pollutant example, one may expect that regions of sparse sampling may have lower pollutant levels than the more densely sampled regions. The first issue refers to the fact that the sample of responses was not obtained uniformly at random over the area of interest, while the second one implies that it may be advantageous for the analysis to take into account the observed locations rather than conditioning on them, as it is done in standard geostatistical methods.

The first contribution of this thesis is to show that previously proposed Monte Carlo (MC) estimates for the likelihood function under preferential sampling found in literature, may not be approximating the desired function. Furthermore, we argue that for preferential sampling of moderate complexity, alternative numerical methods to approximate the likelihood function produce better results than MC methods. We propose the use of Laplace's method to approximate the complex integrals required for the correct likelihood function evaluations in this case.

Research on preferential sampling has so far been restricted to stationary sampling locations such as the monitoring sites mentioned previously, modelled via spatial point processes. One area which has not been considered is the ever growing field of aquatic biomonitoring, in which tagged marine mammals measure ocean properties such as water temperature and salinity. Although the use of animal mounted sensors to analyse population patterns has become ever more common in the last 25 years (Ungar et al., 2005) (Evans et al., 2013), with tags attached to both ocean (Brillinger et al., 2008) and land based (Brillinger et al., 2001) animals, there has also been a move towards the use of these tags to understand the environment in which the animals live. One such example is the use of marine mammal tags to measure oceanographic data. Fedak (2013) discusses the usefulness of such tags in profiling oceanographic data in polar regions, areas which are typical hard to extract data from.

One issue with this type of data however, is that of preferential sampling. With marine mammal mounted tags, one example of this might be the collection of ocean temperature data from an animal whose movement is either directly or indirectly influenced by the water temperature itself. This dependence may follow the common assumption that seals hunt prey in cooler waters and therefore may be inclined to sample lower than average ocean temperatures (Guinet et al., 2014; McIntyre et al., 2011).

The second main contribution of this thesis is to expand preferential sam- 


\subsection{Thesis Outline}

pling methodology for applicability in cases where the sensors are moving through the domain of interest, as is the case with the tagged seals. More specifically, we propose a flexible framework for inference on preferentially sampled fields, where the process that generates the sampling locations is stochastic and moving through a 2-dimensional space. We investigate the effects induced by preferential sampling on parameter estimates and subsequent spatial prediction, with emphasis on estimating ocean temperature fields. Our goal is to reduce the bias in parameter estimates and consequent predictions that preferential sampling may cause in this instance.

\subsection{Thesis Outline}

We begin by providing a background on the topics in this thesis in Chapter 2. This includes an introduction to geostatistics, stochastic process and typical approaches to parameter estimation and spatial prediction. We also introduce spatial point processes and stochastic differential equations which are both used to model the sampling locations in later chapters.

In Chapter 3 we develop the ideas behind preferential sampling in more detail, providing a simulated example of a preferentially sampled point process, which results in parameter estimation and prediction bias when using standard methods.

The first main topic in this thesis is the discussion of previously proposed MC methods for preferential sampling correction and comparison of these with alternative numerical methods. In Chapter 4 we detail the importance of likelihood specification under preferential sampling and how one must account for the dependency between the sampling locations and respective measurements of the spatial phenomenon. We discuss how MC methodology detailed in Diggle et al. (2010) to account for preferential sampling might not be approximating the desired likelihood function and propose that alternative methods such as Laplace approximations implemented using the R package Template Model Builder (TMB) (Kristensen et al., 2016) provide a strong alternative.

In Chapter 5 we discuss the importance of correct specification of the predictive distribution in addition to parameter correction. Naive prediction methods such as kriging do not provide adequate performance even when parameters are corrected for the preferential nature of the sampling. This is because kriging does not utilise information provided by the sampling locations, which may be useful in determining properties of the unknown field in both highly and sparsely covered regions. For example, a sampling design 
that only samples from high temperature regions may provide information as to where the low temperature regions are, due to the lack of sampling in these areas. We study this further in Chapter 6, where we assess the predictive capability of our preferential model in comparison to standard methods for historical ozone data. We also conduct a simulated experiment to compare the effect of preferential sampling and corrective performance across various degrees of sampling preferentiability and sample sizes.

Before expanding our preferential sampling methodology to ocean temperature data collected by marine mammals, we first give a summary of marine mammal movement models in Chapter 7, including examples of various model characteristics. We also show how one may fit certain movement models using TMB and warn against fitting models with non-differentiable likelihood functions which occur with discrete behavioural states. These models are developed further in Chapter 8 with our novel preferential correlated random walk (PCRW) model derived to capture the possible influence of oceanographic factors such as water temperature on marine mammal velocities. In this chapter we also show the possible biases caused by preferential movement of marine mammals and show how our preferential framework developed in Chapters 45 can be used in conjunction with the PCRW to reduce this. We apply our methods to real ocean temperature data collected by Southern elephant seals in the Southern Indian ocean.

Valuable extensions to the work in this thesis are investigated in Chapter 9, such as underwater temperature modelling and introducing varying mean trends in the latent field specification. We then provide concluding remarks and areas of future work which are yet to be fully investigated in Chapter 10 .

Discussions on the computational challenges of simulating high dimensional Gaussian fields required throughout our work can be found in Appendix A, where we offer solutions to the problem applied in our models. This includes using the stochastic partial differential equation (SPDE) approach to obtain a Gaussian Markov random field (GMRF) approximation for our Gaussian field using R-INLA (Integrated Nested Laplace Approximations) (Rue et al., 2009). We also show how we can use output from R-INLA to construct our own sparse precision matrices at higher smoothness levels than those currently supported by the package to efficiently simulate differentiable random surfaces. 


\section{Chapter 2}

\section{Background}

In this chapter we provide a background on the terminology and methods used throughout this thesis. We begin by outlining our geostatistical framework in Section 2.1 before detailing the properties and assumptions of the stochastic processes used throughout our work in Section 2.2. In Section 2.3 we review maximum likelihood and spatial prediction methods used in geostatistics. We discuss various models for sampling locations, highlighting stationary cases using point processes and moving locations via stochastic differential equations in Sections 2.4 and 2.5 respectively.

\section{$2.1 \quad$ Geostatistics}

Geostatistics is the framework used to provide "quantitative descriptions of natural variables distributed in space or in time and space" (Chiles and Delfiner, 2009). In general, the aim of the statistician is to both understand the properties and predict at unobserved locations a spatial phenomenon $\left\{S(\mathbf{X}): \mathbf{X} \in \mathcal{D} \subseteq \mathbb{R}^{2}\right\}$ measured at a discrete set of locations in some domain $\mathcal{D}$, where in general the process $S$ cannot be measured without error (Diggle et al., 1998). Applications include climatology such as temperature (Góis et al., 2010) and pollutant modelling (Shaddick and Zidek, 2014), and spatial epidemiology which studies the spatial properties of disease and mortality (Goovaerts, 2008).

A flexible and widely used model postulates that the observations $Y_{i}$ are given by

$$
Y_{i}=S\left(\mathbf{X}_{i}\right)+\epsilon_{i}, \quad \mathbf{X}_{i} \in \mathcal{D}, \quad i=1, \ldots, n,
$$

where $S$ has some mean function $\mu: \mathcal{D} \rightarrow \mathbb{R}, \mathbf{X}_{i}$ are the measurement locations and $\epsilon_{i}$ are mutually independent random errors with mean 0 and so called "nugget variance" $\tau^{2}$. 


\subsection{Stochastic Processes}

We begin by defining the terminology that will enable us to build a realistic model framework of the form in (2.1). This starts with a stochastic process which is defined as collection of random variables $\left\{\mathbf{X}_{j}: j \in \mathcal{T}\right\}$ for some index set $\mathcal{T}$.

\subsubsection{Spatial Gaussian Processes and Fields}

If $S(\mathbf{X})$ is a random variable for any $\mathbf{X} \in \mathcal{D} \subseteq \mathbb{R}^{2}$, then we say the collection of random variables $\{S(\mathbf{X}): \mathbf{X} \in \mathcal{D}\}$ is a spatial stochastic process on some spatial domain $\mathcal{D}$. We say a spatial stochastic process $S: \mathcal{D} \rightarrow \mathbb{R}$ on a 2-dimensional spatial domain $\mathcal{D}$ is a spatial Gaussian process with mean function $\mu: \mathcal{D} \rightarrow \mathbb{R}$ and covariance function $\sigma: \mathcal{D} \times \mathcal{D} \rightarrow \mathbb{R}_{\geq 0}$, if for any vector $\mathbf{X}=\left(\mathbf{X}_{1}, \ldots, \mathbf{X}_{n}\right)^{T}$ such that all $\mathbf{X}_{i} \in \mathcal{D}$, the random vector $S=$ $\left(S\left(\mathbf{X}_{1}\right), \ldots, S\left(\mathbf{X}_{n}\right)\right)^{T}$ has an $n$-dimensional multivariate normal distribution $S \sim N_{n}\left(\boldsymbol{\mu}_{G P}, \boldsymbol{\Sigma}_{G P}\right)$ where:

- The $n$-dimensional mean vector is defined $\boldsymbol{\mu}_{G P}=\left(\mu\left(\mathbf{X}_{1}\right), \ldots, \mu\left(\mathbf{X}_{n}\right)\right)^{T}$.

- The $n \times n$ covariance matrix is defined

$$
\mathbf{\Sigma}_{G P}=\left[\begin{array}{cccc}
\sigma\left(\mathbf{X}_{1}, \mathbf{X}_{1}\right) & \sigma\left(\mathbf{X}_{1}, \mathbf{X}_{2}\right) & \cdots & \sigma\left(\mathbf{X}_{1}, \mathbf{X}_{n}\right) \\
\sigma\left(\mathbf{X}_{2}, \mathbf{X}_{1}\right) & \sigma\left(\mathbf{X}_{2}, \mathbf{X}_{2}\right) & \cdots & \sigma\left(\mathbf{X}_{2}, \mathbf{X}_{n}\right) \\
\vdots & \vdots & & \vdots \\
\sigma\left(\mathbf{X}_{n}, \mathbf{X}_{1}\right) & \sigma\left(\mathbf{X}_{n}, \mathbf{X}_{2}\right) & \cdots & \sigma\left(\mathbf{X}_{n}, \mathbf{X}_{n}\right)
\end{array}\right]
$$

where the covariance function is such that $\sigma\left(\mathbf{X}_{i}, \mathbf{X}_{j}\right)$ is the covariance between $S\left(\mathbf{X}_{i}\right)$ and $S\left(\mathbf{X}_{j}\right)$ for any $\mathbf{X}_{i}, \mathbf{X}_{j} \in \mathcal{D}$. The covariance matrix $\Sigma_{G P}$ must be positive-semidefinite, ie: $\sum_{i, j}^{n} \alpha_{i} \alpha_{j} \sigma\left(\mathbf{X}_{i}, \mathbf{X}_{j}\right) \geq 0$ for any $\alpha_{1}, \ldots, \alpha_{n} \in \mathbb{R}$.

The attractive nature of Gaussian processes in specifying random surfaces is largely due to the well-known marginal and conditional multivariate normal distributions which are entirely specified through the mean and covariance functions (Gelfand and Schliep, 2016). Spatial Gaussian processes are often referred to as Gaussian fields when taken across an entire spatial region in Euclidean space. In the context of this thesis will interchange their usage. 


\subsubsection{Stationarity}

We say a Gaussian process $S$ is second-order stationary if and only if for all $\mathbf{X} \in \mathcal{D}$

$$
\begin{aligned}
\mathbb{E}[S(\mathbf{X})] & =\mu(\mathbf{X})=c, \\
\operatorname{Cov}(S(\mathbf{X}), S(\mathbf{X}+\mathbf{h})) & =\sigma(\mathbf{h}),
\end{aligned}
$$

where $\mu: \mathcal{D} \rightarrow \mathbb{R}$ and $\sigma: \mathcal{D} \times \mathcal{D} \rightarrow \mathbb{R}_{\geq 0}$ are the mean and covariance functions respectively and $c \in \mathbb{R}, \mathbf{h} \in \mathbb{R}^{2}$. A Gaussian process that is secondorder stationary is also strictly stationary, which is the property of having joint distributions which do not change when shifted in space. However, this property is dependent on the Gaussian assumption (Gelfand et al., 2010).

A more specific type of second-order stationarity is the case in which

$$
\operatorname{Cov}(S(\mathbf{X}), S(\mathbf{X}+\mathbf{h}))=\sigma(\|\mathbf{h}\|),
$$

where $\|\cdot\|$ is the Euclidean distance. In this case the process $S$ is called isotropic. We follow the language of Diggle and Ribeiro (2007) and refer to any process $S$ that is both second-order stationary and isotropic as simply a stationary process. In this case, the mean of the stochastic process is constant across the entire domain (stationary in the mean) and the covariance between two locations depends only on the Euclidean distance between them (isotropic).

In what follows we will be assuming that our spatial fields are stationary, but the methods proposed can likely be extended to non-stationary equivalents. Sampson (2010) provides a useful summary of techniques used to model spatial processes with non-stationary covariance structures that may vary over time and space. These include but are not limited to process convolution models which use a moving average Gaussian process in which the dependence structure may change over space (Higdon, 1998) and the spatial deformation approach in which coordinates in a non-stationary geographical space are mapped via a smooth thin-plate spline to stationary deformed space (Sampson and Guttorp, 1992). Stationary in the mean is discussed in further detail in Section 9.2 .

\subsubsection{Continuity and Differentiability}

The continuity and differentiability of stochastic processes are entirely dependent on their covariance structure. There are two definitions of continuity and differentiability, namely path and mean-square. We say a process 
$S(\mathbf{X})$ is path continuous or path differentiable if realisations of the process are continuous or differentiable functions respectively (Diggle and Ribeiro, 2007). We say a process $S(\mathbf{X})$ is mean-square continuous if

$$
\mathbb{E}\left[\{S(\mathbf{X}+\mathbf{h})-S(\mathbf{X})\}^{2}\right] \rightarrow 0,
$$

as $\mathbf{h} \rightarrow 0$. Similarly, we say a process $S(\mathbf{X})$ is mean-square differentiable with derivative $S^{\prime}(\mathbf{X})$ if

$$
\mathbb{E}\left[\left\{\frac{S(\mathbf{X}+\mathbf{h})-S(\mathbf{X})}{\mathbf{h}}-S^{\prime}(\mathbf{X})\right\}^{2}\right] \rightarrow 0,
$$

as $\mathbf{h} \rightarrow 0$. Although path properties are the stronger of the two forms (Kent et al., 1989), it is common to work with the mean-square definition due to its direct relationship with the existence of derivatives of the covariance function (Adler, 1981).

\subsubsection{Covariance Families}

It is difficult to find parametric functions that satisfy the properties of a covariance function, namely positive definitiveness, whilst also maintaining flexibility in their structure to account for various continuity and differentiability properties (Diggle and Ribeiro, 2007). In general, statisticians choose from a set of regularly used stationary covariance families including non-monotone, Exponential and Matérn. Each family contains their own distinct set of correlation properties such as the oscillatory nature of the non-monotone covariance function and the possibility of infinite meansquare differentiability in the Exponential family.

The most commonly used covariance family is the Matérn family (Matérn, 1960), largely due to the flexibility in adopting various levels of smoothness and consequent levels of mean-square differentiability and continuity. We define the Matérn covariance function between $S(\mathbf{X})$ and $S\left(\mathbf{X}^{\prime}\right)$ where $u=\left\|\mathbf{X}-\mathbf{X}^{\prime}\right\|$ as

$$
\rho(u)=\sigma^{2} \frac{2^{1-\kappa}}{\Gamma(\kappa)}\left(\frac{u}{\phi}\right)^{\kappa} K_{\kappa}\left(\frac{u}{\phi}\right),
$$

where $\Gamma$ is the gamma function, $K_{\kappa}$ is the modified Bessel function of the second kind, $\sigma^{2}$ is the marginal variance (partial sill) of the process and $\kappa, \phi \in \mathbb{R}^{>0}$ are the smoothness and scale parameters. 
Defining $\lceil x\rceil$ as the smallest integer greater than or equal to $x$, we have that a Gaussian process with Matérn covariance is $\lceil\kappa-1\rceil$ mean-square differentiable, hence by varying $\kappa$ we can directly alter the smoothness properties of the process. Setting $\kappa \in(0,1]$ we have a mean-square continuous process that is not mean-square differentiable, however any $\kappa>1$ will result in a process that is at least once mean-square differentiable (Banerjee and Gelfand, 2003; Banerjee et al., 2003). Meanwhile the scale parameter $\phi$ controls the rate of correlation decay as $u$ increases.

One drawback of the Matérn function is the non-orthogonality between $\kappa$ and $\phi$. In other words, for a true Matérn covariance structure with parameters $\kappa$ and $\phi$, the closest approximation with an alternative smoothness parameter $\kappa^{\prime} \neq \kappa$, is to use a scale $\phi^{\prime} \neq \phi$ (Diggle and Ribeiro, 2007). Hence it is not possible to directly compare scale $\phi$ across different values of $\kappa$. This makes it difficult to optimise over the Matérn parameter space and in applications we usually assume the smoothness $\kappa$ to be known, or only allowed to vary over a discrete domain.

\subsection{Maximum Likelihood Estimation and Spatial Prediction}

\subsubsection{Maximum Likelihood Estimation}

In most geostatistical literature, models such as those described in (2.1) treat the sampling locations, $\mathbf{X}_{i}$ 's, as deterministic or stochastically independent from the process being measured and therefore analysis on the spatial field is based on the unconditional distribution of $\mathbf{Y}=\left(Y_{1}, \ldots, Y_{n}\right)$ (Diggle et al., 2010). When the data is also assumed Gaussian, maximum likelihood estimation is fairly simple method to obtain parameter estimates of the mean and spatial covariance structure. Assuming we have a spatial trend surface $\mu(\mathbf{X})=\mathbf{D}_{n} \boldsymbol{\zeta}$ where $\mathbf{D}_{n}$ is an $n \times p$ matrix of covariates and $\boldsymbol{\zeta}$ an $p$-dimensional vector of regression coefficients, then a Gaussian model for data is

$$
\mathbf{Y}=N_{n}\left(\mathbf{D}_{n} \boldsymbol{\zeta}, \Sigma(\boldsymbol{\eta})+\tau^{2} I_{n}\right)
$$

where $\Sigma$ is an $n \times n$ covariance matrix of the field $S$ at the sampled locations and $I_{n}$ an $n$-dimensional identity matrix. The vector of covariance parameters $\boldsymbol{\eta}$ depend on the choice of covariance function and nugget parameter $\tau^{2}$ accounts for measurement error in the observations of the response $\mathbf{Y}$. We can then estimate the full set of field parameters, denoted $\boldsymbol{\theta}=\left(\boldsymbol{\zeta}, \boldsymbol{\eta}, \tau^{2}\right)$, 
using the log-likelihood function

$$
\begin{array}{r}
L(\boldsymbol{\theta})=[\mathbf{Y}]=-\frac{1}{2}\left(\log \left|\Sigma(\boldsymbol{\eta})+\tau^{2} I_{n}\right|+n \log (2 \pi)+\right. \\
\left.\log \left\{\left(\mathbf{y}-\mathbf{D}_{n} \boldsymbol{\zeta}\right)^{T}\left(\Sigma(\boldsymbol{\eta})+\tau^{2} I_{n}\right)^{-1}\left(\mathbf{y}-\mathbf{D}_{n} \boldsymbol{\zeta}\right)\right\}\right),
\end{array}
$$

for some realisations $\mathbf{y}=\left(y_{1}, \ldots, y_{n}\right)$.

In the case for which we cannot use the model in 2.5, such as if we retract the Gaussian assumption, then estimating the parameter vector $\boldsymbol{\theta}$ becomes a more complicated problem. This requires us to explicitly state the distributions involved in evaluating the likelihood function

$$
L(\boldsymbol{\theta})=\int[\mathbf{Y} \mid S][S] \mathrm{d} S,
$$

where we use the notation of $[W]$ to denote the density or probability mass function of the random object $W$. Note that in this case we are still assuming the sampling locations, $\mathbf{X}$, as deterministic and hence retracted from the model definition.

The integral in (2.7) can be broken down into two parts. First we can treat the conditional density $[\mathbf{Y} \mid S]$ as a product of univariate densities $[\mathbf{Y} \mid S]=\prod_{i=1}^{n}\left[\mathbf{Y}_{i} \mid S\right]$ due to the conditional independence assumption of the measurements $\mathbf{Y}$ given the latent field $S$. Hence we can re-write (2.7) as

$$
L(\boldsymbol{\theta})=\int \prod_{i=1}^{n}\left[\mathbf{Y}_{i} \mid S\right][S] \mathrm{d} S .
$$

The marginal density $[S]$ is tricky to manage in a spatial setting. Since the $S\left(\mathbf{X}_{i}\right)$ 's are not mutually independent due to their spatial correlation, we are forced to evaluate the high-dimensional density $[\mathbf{S}]$ of the variable $\mathbf{S}=$ $\left(S\left(\mathbf{X}_{1}\right), \ldots, S\left(\mathbf{X}_{n}\right)\right)$. In this case we may use Monte Carlo (MC) simulations to approximate the likelihood by

$$
\begin{aligned}
L(\boldsymbol{\theta}) & \approx \int \prod_{i=1}^{n}\left[\mathbf{Y}_{i} \mid \mathbf{S}\right][\mathbf{S}] \mathrm{d} \mathbf{S} \\
& =\mathbb{E}_{\mathbf{S}}\left[\prod_{i=1}^{n}\left[\mathbf{Y}_{i} \mid \mathbf{S}\right]\right] \\
& \approx \frac{1}{m} \sum_{j=1}^{m}\left[\prod_{i=1}^{n}\left[\mathbf{Y}_{i} \mid \mathbf{S}_{j}\right]\right]
\end{aligned}
$$


where the $n$-dimensional vectors $\mathbf{S}_{j}$ are realisations of $\mathbf{S}$. The issues here are two fold. Firstly, simulating the $\mathbf{S}_{j}$ 's can be computationally inefficient, particularly in large data sets, since the dimension of $\mathbf{S}$ is also forced to be big. Secondly, simulating independent realisations of $\mathbf{S}_{j}$ for each MC simulation is extremely inefficient. This is because of the large variation possible between each $\mathbf{S}_{j}$ exacerbated by the spatial correlation within each vector. Therefore, it is extremely rare for a vector $\mathbf{S}_{j}$ to be compatible with the data $\mathbf{Y}$. These two issues combined make naive MC simulations unrealistic in a practical setting and variance reduction techniques a necessity (Diggle and Ribeiro, 2007).

One option is the use of antithetic pairs. If we sample a pair of variables that follow the same distribution, say $\mathbf{S}$ and $\mathbf{S}^{*}$ in $(2.9)$, then

$$
\mathbb{E}\left[\frac{1}{m} \sum_{j=1}^{m / 2}\left(\left[\mathbf{Y} \mid \mathbf{S}_{j}\right]+\left[\mathbf{Y} \mid \mathbf{S}_{j}^{*}\right]\right)\right]=\mathbb{E}\left[\frac{1}{m} \sum_{j=1}^{m}\left[\mathbf{Y} \mid \mathbf{S}_{j}\right]\right],
$$

and

$$
\begin{aligned}
\operatorname{Var}\left(\frac{1}{m} \sum_{j=1}^{m / 2}\left(\left[\mathbf{Y} \mid \mathbf{S}_{j}\right]+\left[\mathbf{Y} \mid \mathbf{S}_{j}^{*}\right]\right)\right) & =\frac{1}{2 m} \operatorname{Var}\left([\mathbf{Y} \mid \mathbf{S}]+\left[\mathbf{Y} \mid \mathbf{S}^{*}\right]\right) \\
& =\frac{\operatorname{Var}([\mathbf{Y} \mid \mathbf{S}])}{m}\left(1+\operatorname{Cor}\left([\mathbf{Y} \mid \mathbf{S}],\left[\mathbf{Y} \mid \mathbf{S}^{*}\right]\right)\right) \\
& =\frac{\sigma^{2}}{m}(1+\rho) .
\end{aligned}
$$

Hence if we can generate variables such that $\rho<0$ we have a reduction in the variance of the MC simulation process. Ripley (2009) discusses methods to obtain effective antithetic pairs that minimise $\rho$, which depends on both the symmetry and complexity of the distributions at hand.

Another option is importance sampling. In this case we change the distribution with which we take the expectation. Using $(2.8)$ as an example, we can write

$$
L(\boldsymbol{\theta})=\int[\mathbf{Y} \mid S][S] \mathrm{d} S=\int \frac{[\mathbf{Y} \mid S][S] p(S)}{p(S)} \mathrm{d} S=\mathbb{E}_{p}\left[\frac{[\mathbf{Y} \mid S][S]}{p(S)}\right],
$$

where $p$ is a probability density function such that $p(S)=0 \Longrightarrow[\mathbf{Y} \mid S][S]=$ 0 . The aim is to choose an importance sampler $p$ such that draws of $S$ from this distribution have a reduced MC variability in the estimates of the likelihood, hereby improving computational efficiency. Robert and Casella (2004) 
and Rubinstein and Kroese (2017) among many others provide further information on importance sampling techniques, including details on how to choose effective importance distributions for a given experiment. In the case of (2.9) we may wish to use an importance sampler to generate $\mathbf{S}_{j}$ 's which are more compatible with $\mathbf{Y} \mid \mathbf{S}$ than the naive samples from $\mathbf{S}$. One naive choice could be to choose $p(\mathbf{S})=[\mathbf{S} \mid \mathbf{Y}]$ and hence use the alternative likelihood form

$$
L(\boldsymbol{\theta})=\mathbb{E}_{\mathbf{S} \mid \mathbf{Y}}\left[\frac{[\mathbf{Y} \mid \mathbf{S}][\mathbf{S}]}{[\mathbf{S} \mid \mathbf{Y}]}\right] .
$$

In this case we would expect samples from $\mathbf{S} \mid \mathbf{Y}$ to be more compatible with $\mathbf{Y} \mid \mathbf{S}$ and reduce the MC variability across simulations, improving computational efficiency. However using $[\mathbf{S} \mid \mathbf{Y}]$ as our importance sampler in this case simply reduces the inside of the expectation to a constant since

$$
\frac{[\mathbf{Y} \mid \mathbf{S}][\mathbf{S}]}{[\mathbf{S} \mid \mathbf{Y}]}=\frac{[\mathbf{Y}, \mathbf{S}]}{[\mathbf{S} \mid \mathbf{Y}]}=[\mathbf{Y}]
$$

This is an issue we refer to again when discussing preferential sampling MC methodology in Section 4.3.3.

\subsubsection{Spatial Prediction}

One of the main aims of a spatial statistician is to predict the latent, unobservable field $S$ at unsampled locations. Say that, as previously, we have our data consisting of sampling locations $\mathbf{X}=\left(\mathbf{X}_{1}, \ldots, \mathbf{X}_{n}\right)$ and observations of the latent field $\mathbf{Y}=\left(Y_{1}, \ldots, Y_{n}\right)$ according to the model in (2.1), measured with or without error. Using this data we want to predict the field at a location $\mathbf{u} \in \mathcal{D}$, denoted $T=S(\mathbf{u})$. Following Diggle and Ribeiro (2007) and assuming the sampling locations are non-random, a point predictor for $T$ is a function of the observations $\hat{T}=f(\mathbf{Y})$. Then the minimum mean square error predictor is

$$
\hat{T}=\mathbb{E}[T \mid \mathbf{Y}] .
$$

Assuming the field $S$ is a stationary Gaussian process we can derive the distribution $T \mid \mathbf{Y}$ via the properties of the multivariate conditional Gaussian. Assuming $\mathbf{Y} \sim N_{n}\left(\mu \mathbf{1}, \Sigma+\tau^{2} I_{n}\right)$ then $(T, \mathbf{Y})$ is multivariate Gaussian with mean vector $(\mu, \mu \mathbf{1})^{\top}$ and covariance matrix

$$
\Sigma=\left[\begin{array}{cc}
\sigma^{2} & \sigma^{2} \mathbf{r}^{\top} \\
\sigma^{2} \mathbf{r} & \Sigma+\tau^{2} I_{n}
\end{array}\right]
$$

where $r_{i}=\rho\left(\left\|\mathbf{u}-\mathbf{X}_{i}\right\|\right)$ is the $i$ th element of the vector $\mathbf{r}$. 
Hence we have that the minimum mean square estimator is

$$
\hat{T}=\mathbb{E}[T \mid \mathbf{Y}]=\mu+\sigma^{2} \mathbf{r}^{\top}\left(\Sigma+\tau^{2} I_{n}\right)^{-1}(\mathbf{Y}-\mu \mathbf{1}),
$$

with prediction variance

$$
\operatorname{Var}(T \mid \mathbf{Y})=\sigma^{2}\left(1-\sigma^{2} \mathbf{r}^{\top}\left(\Sigma+\tau^{2} I_{n}\right)^{-1} \mathbf{r}\right)
$$

derived using properties of the conditional normal distribution. This prediction technique is called kriging, a name coined by Matheron (1963) named after the work of Krige (1951) in statistical analyses of gold core-samples in South Africa (Cressie, 1990).

We can rewrite the kriging estimator as

$$
\begin{aligned}
\hat{T} & =\mu+\sum_{i=1}^{n} w_{i}(\mathbf{u})\left(Y_{i}-\mu\right), \\
& =\mu\left(1-\sum_{i=1}^{n} w_{i}(\mathbf{u})\right)+\sum_{i=1}^{n} w_{i}(\mathbf{u}) Y_{i},
\end{aligned}
$$

where $w_{i}$ is the $i$ th element of an $n$-dimensional vector $\mathbf{w}(\mathbf{u})=\sigma^{2} \mathbf{r}^{\top}(\Sigma+$ $\left.\tau^{2} I_{n}\right)^{-1}$. Therefore we can view the kriging predictor as taking a weighted balance between the mean parameter $\mu$ and the observed data $\mathbf{Y}$. The predictions of $T=S(\mathbf{u})$ depend on both the location of $\mathbf{u}$ with respect to the sampling locations $\mathbf{X}$, but also the relationship between the sampling locations. For example, two sampling locations close to each other rarely provide twice the information of a single observation in the same region, although this depends on the covariance parameters. This effect is accounted for via the inclusion of $\Sigma$ in the prediction weights, w, which adjusts weights of samples from highly correlated sampling locations. Meanwhile, the prediction variance does not depend on $\mathbf{Y}$, only the sampling locations and covariance parameters of the model.

Using these kriging predictors requires the use of so-called "plug-in" parameters. If the mean parameter $\mu$ is assumed known or estimated as the sample mean of the data, then we refer to this as simple kriging. If the mean parameter is assumed unknown and replaced by its generalised least squares estimator

$$
\hat{\mu}=\left(\mathbf{1}^{\top}\left(\Sigma+\tau^{2} I_{n}\right)^{-1} \mathbf{1}\right)^{-1} \mathbf{1}^{\top}\left(\Sigma+\tau^{2} I_{n}\right)^{-1} \mathbf{Y},
$$

then we call this ordinary kriging. Ordinary kriging is equivalent to estimating the mean and covariance parameters together by maximising $(2.6)$ in the classical maximum likelihood estimation method outlined in Section 2.3.1. 
The spatially varying mean alternative, where $\mu(\mathbf{u})=\mathbf{D}_{u} \boldsymbol{\zeta}$ with $\mathbf{D}_{u}$ a $1 \times p$ matrix of covariates and $\boldsymbol{\zeta}$ a $p$-dimensional vector of regression coefficients similar to $(2.5)$, is called univerisal kriging. In this case

$$
\hat{T}=\mathbf{D}_{u} \boldsymbol{\zeta}+\sum_{i=1}^{n} w_{i}(\mathbf{u})\left(Y_{i}-\mathbf{D}_{i} \boldsymbol{\zeta}\right)
$$

where $\mathbf{D}_{i}$ is the $i$ th row of the $n \times p$ covariate matrix $\mathbf{D}_{n}$ from $(2.5)$ and as previously $w_{i}$ is the $i$ th element of an $n$-dimensional vector $\mathbf{w}(\mathbf{u})=\sigma^{2} \mathbf{r}^{\top}(\Sigma+$ $\left.\tau^{2} I_{n}\right)^{-1}$.

Deriving $\mathbb{E}[T \mid \mathbf{Y}]$ and $\operatorname{Var}(T \mid \mathbf{Y})$ can be more complex if we retract the Gaussianity assumption, or more importantly for the work in this thesis, the assumption of independence between the measurements $\mathbf{Y}$ and sampling locations $\mathbf{X}$. This is the issue of preferential sampling which we discuss in Chapter 3 .

\subsection{Spatial Point Processes}

Spatial point processes are statistical models for random point patterns in some $d$-dimensional space, where most often we are interested in the case $d=2$ or $d=3$ (Baddeley et al., 2006). In most cases we are interested in modelling the locations of these point patterns to try and understand the process that is driving their spatial distribution. Examples of point pattern usage in 2-dimensions include assessing the interactions between ant nest locations for different species (Högmander and Särkkä, 1999), relating patterns of tree species location to environmental variables (Møller and Waagepetersen, 2007) and even the modelling of fixation locations of the eye on photographs (Barthelmé et al., 2013). In other cases we might wish to use point patterns to model the locations at which measurements of some spatial phenomenon are made. In most instances this factor is ignored when analysing spatial data by considering the sampling locations fixed (Diggle and Ribeiro, 2007). However, as we introduce in Section 3 this assumption may be misleading in cases which the sampling locations are dependent on the process we are measuring.

Mathematically we define a spatial point process $\mathbf{X}$ in 2 dimensions as a finite random subset of some region $\mathcal{D} \subseteq \mathbb{R}^{2}$, such that any realisation of this process $\mathbf{x}=\left(\mathbf{x}_{1}, \ldots \mathbf{x}_{n}\right)^{T}$ where $n \geq 0$ is contained in $\mathcal{D}$. In this case we call $\mathbf{x}$ a spatial point pattern and $\mathbf{X}$ a spatial point process on $\mathcal{D}(\mathrm{M} \varnothing$ ller and Waagepetersen, 2007). 
Spatial point processes are specified by an intensity function which defines the spatial properties of the system. Some models may require intensity functions that induce an interaction between points such as clustering and are commonly modelled via Log Gaussian Cox processes (Møller et al., 1998). These are called aggregated point patterns. In other cases interactions might even be required to repel points in close proximity, modelled via methods such as determinental point processes (Lavancier et al., 2015).

Intensity functions which do not include interactions are commonly modelled via Poisson point processes which can be further broken down into homogeneous and non-homogeneous processes depending on whether or not the intensity is a function of spatial location. A Poisson point process $X$ on $\mathcal{D} \subseteq \mathbb{R}^{2}$ with intensity function $\lambda: \mathcal{D} \rightarrow \mathbb{R}^{>0}$ is a point process such that:

1. The number of points in any $\mathcal{A} \subseteq \mathcal{D}$, denoted $N(\mathcal{A})$, follows a Poisson distribution with mean $\lambda(\mathcal{A})=\int_{\mathcal{A}} \lambda(x) \mathrm{d} x$,

2. For any finite collection of pairwise disjoint $\mathcal{A}_{1} \ldots, \mathcal{A}_{n} \subseteq \mathcal{D}$, the number of points in each region $N\left(\mathcal{A}_{1}\right) \ldots, N\left(\mathcal{A}_{n}\right)$ are independent random variables.

When $\lambda(x)=c$ for all $x \in \mathcal{D}$ for some constant $c \in \mathbb{R}^{>0}$, we have a homogeneous Poisson process, otherwise we have an inhomogeneous Poisson process.

\subsection{Stochastic Differential Equations}

An alternative model for the sampling locations is that of stochastic differential equations (SDEs). In this case the sampling locations are not point patterns but discretely observed locations from a continuously moving process which we will restrict to 2-dimensions. In this instance, sampling locations are not independent conditional on the random field since the observed location at time $t+1$ will depend on the location at time $t$.

The modelling of dynamic movement using SDEs is covered in great detail in the works of David Brillinger, with some examples of note including Brillinger et al. (2001); Brillinger and Stewart (1998); Brillinger et al. (2008). These papers discuss various methods and applications for implementing a stochastic framework to analyse the movement of an animal. That is, we model the change in location of the animal at time $t$ using the following SDE

$$
\mathrm{d} \mathbf{X}(t)=\boldsymbol{\mu}(\mathbf{X}(t), \boldsymbol{\theta}) \mathrm{dt}+\boldsymbol{\Sigma}(\boldsymbol{\theta}) \mathrm{d} \boldsymbol{B}(t),
$$


where $\mathbf{X}(t) \in \mathbb{R}^{2}$ is the location of the animal at time $t$. The 2 by 2 matrix $\boldsymbol{\Sigma}$ is known as the diffusion term and accounts for uncertainty in the movement of the animal by incremental Brownian motion $\boldsymbol{B}(t)$, which can be thought of as Gaussian white noise. We denote the vector of model parameters as $\boldsymbol{\theta}$.

The function $\boldsymbol{\mu}: \mathbb{R}^{2} \rightarrow \mathbb{R}^{2}$ is called the drift function and has the mathematical interpretation

$$
\mathbb{E}\left[\mathrm{d} \mathbf{X}(t) \mid M_{t}\right] \approx \boldsymbol{\mu}(\mathbf{X}(t), \boldsymbol{\theta}) \mathrm{d} t,
$$

such that $M_{t}=\{\mathbf{X}(u): u \leq t\}$ denotes the history of the process up to and including time $t$ (Brillinger, 2003). This is interpreted as the expected instantaneous movement velocity at time $t$ given all previously known locations.

Although Brillinger uses various parametric and non-parametric structures for $\boldsymbol{\mu}(\cdot)$ to model the expected movement of animals at particular locations in time and space, an alternative method is to model a 3-dimensional surface known as the potential field. The potential field is the surface obtained when we evaluate some function $H: \mathcal{D} \rightarrow \mathbb{R}$, called the potential function, across some 2-dimensional domain $\mathcal{D} \subseteq \mathbb{R}^{2}$. The potential field is another means to visualise the expected spatial movement of an animal, via a 3-dimensional surface. One can imagine the expected movement of an animal at a particular location in the $x y$-plane to be in the direction that a ball would roll in the $x y$-plane when placed on the surface of the potential field at that point. In other words, observing the movement of the ball on a 3 -dimensional surface from a birds-eye view. Mathematically the potential function $H$ at location $\mathbf{X}(t)$ is defined:

$$
\boldsymbol{\mu}(\mathbf{X}(t), \boldsymbol{\theta})=-\left.\nabla_{\mathbf{X}} H(\mathbf{X}, \boldsymbol{\theta})\right|_{\mathbf{X}=\mathbf{X}(t)} .
$$

An example of the potential function $H(\mathbf{X}(t), \boldsymbol{\theta})=X_{1}(t)^{2}+X_{2}(t)^{2}$ and respective drift function $\boldsymbol{\mu}(\mathbf{X}(t), \boldsymbol{\theta})=-2\left(X_{1}(t), X_{2}(t)\right)^{\top}$ can be seen in Figure 2.1. In this example the potential function shows that the expected movement of an animal in this domain is always towards the center point $(0,0)$, since a ball placed anywhere on the surface would head in that direction. The respective drift function on the right hand side of this plot also shows this. The directions of the arrows represent the direction of the drift function at that point, whilst the length of the arrow tails represent the speed of the movement, which decreases the closer to the center you get. In this example, the expected movement of the animal does not depend on time, an assumption which is maintained throughout the work in this paper. 


\subsection{Stochastic Differential Equations}
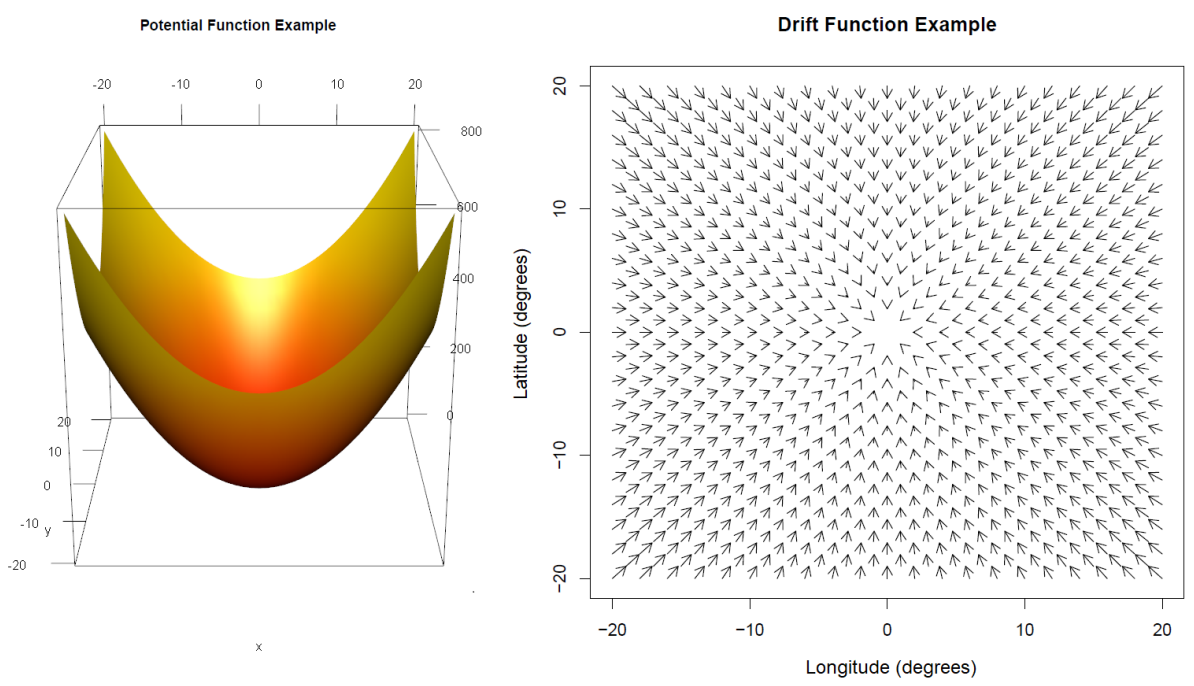

Figure 2.1: Left Panel: Example of the potential function $H(\mathbf{X}(t), \boldsymbol{\theta})=$ $X_{1}(t)^{2}+X_{2}(t)^{2}$. Right Panel: The respective drift function $\boldsymbol{\mu}(\mathbf{X}(t), \boldsymbol{\theta})=$ $-2\left(X_{1}(t), X_{2}(t)\right)^{\top}$. 


\section{Chapter 3}

\section{Preferential Sampling}

In this chapter we outline the phenomenon know as preferential sampling, giving an introduction in Section 3.1, which includes a literature review and a discussion of various instances in which it may occur. We introduce preferential sampling of point processes in Section 3.2, providing simulated examples of the phenomenon and also discussing the drawbacks of our particular point process model. Lastly, in Section 3.3 we introduce the notion of preferential sampling of data obtained from a process moving in some 2-dimensional space.

\subsection{Preferential Sampling Overview}

In most literature, geostatistical models such as those described in (2.1) treat the sampling locations, $\mathbf{X}_{i}$ 's, as fixed and therefore analysis on the spatial field is based on the unconditional distribution of $\mathbf{Y}=\left(Y_{1}, \ldots, Y_{n}\right)$. In the instance that the locations are considered stochastic, $\mathbf{X}=\left(\mathbf{X}_{1}, \ldots, \mathbf{X}_{n}\right)$, then they are generally assumed independent of $S$. In these geostatistical models, since the process that selects the locations $\mathbf{X}$ at which observations are made is assumed to be independent from the response process $S$, we have $[\mathbf{X} \mid S]=[\mathbf{X}]$ and thus $[\mathbf{X}, \mathbf{Y}, S]=[\mathbf{Y} \mid S, \mathbf{X}][\mathbf{X}][S]$. In this case, denoting $\boldsymbol{\theta}$ as the full set of field parameters, it follows that

$$
L(\boldsymbol{\theta})=[\mathbf{X}][\mathbf{Y} \mid \mathbf{X}],
$$

and inference about $\boldsymbol{\theta}$ can be carried out conditionally on the observed locations $\mathbf{X}$. Implicit in this argument is that $[\mathbf{X}]$ does not depend on the parameters of interest $\boldsymbol{\theta}$. 
We derive the likelihood in (3.1) by first observing that in this case

$$
\begin{aligned}
{[\mathbf{Y} \mid \mathbf{X}] } & =\int[\mathbf{Y}, S \mid \mathbf{X}] \mathrm{d} S, \\
& =\int[\mathbf{Y} \mid S, \mathbf{X}][S \mid \mathbf{X}] \mathrm{d} S, \\
& =\int[\mathbf{Y} \mid S, \mathbf{X}][S] \mathrm{d} S,
\end{aligned}
$$

and therefore

$$
\begin{aligned}
L(\boldsymbol{\theta}) & =\int[\mathbf{X}, \mathbf{Y}, S] \mathrm{d} S, \\
& =\int[\mathbf{Y} \mid S, \mathbf{X}][\mathbf{X}][S] \mathrm{d} S, \\
& =[\mathbf{X}] \int[\mathbf{Y} \mid S, \mathbf{X}][S] \mathrm{d} S, \\
& =[\mathbf{X}][\mathbf{Y} \mid \mathbf{X}] .
\end{aligned}
$$

The final line uses the fact that $[\mathbf{Y} \mid \mathbf{X}]=\int[\mathbf{Y} \mid S, \mathbf{X}][S] \mathrm{d} S$ from (3.2).

Preferential sampling refers to the situation where the observed locations $\mathbf{X}$ may depend on the unobserved process $S$. In symbols: we are concerned with the situation where $[\mathbf{X} \mid S] \neq[\mathbf{X}]$. In this case, the arguments above do not hold and to perform inference on $\boldsymbol{\theta}$ one should use the full likelihood function:

$$
L(\boldsymbol{\theta})=\int[\mathbf{X}, \mathbf{Y}, S] \mathrm{d} S=\int[\mathbf{Y} \mid S, \mathbf{X}][\mathbf{X} \mid S][S] \mathrm{d} S .
$$

If ignored, preferential sampling may lead to biased parameter estimates and spatial prediction. For example, consider data in which the random process $S$ is only sampled in regions which contain the larger-values of $S$. If we ignore this in the subsequent analysis then one might expect overestimation in the mean parameter and subsequent spatial prediction. Furthermore, the sampling locations provide information for the spatial prediction which is ignored if we use the kriging method from Section 2.3.2. Kriging naively extrapolates predictions based on the estimates of spatial correlation and other field parameters, whereas if we identified the relationship between sampling locations and the field we might be able to predict that sparsely sampled regions are likely to have lower values of $S$ in comparison to the highly sampled regions. 


\subsection{Preferential Sampling Overview}

The impact of preferential sampling on estimation and prediction has been discussed in various recent papers. Shaddick and Zidek (2014) highlighted the preferential nature by which air pollution monitoring sites were added and removed from a UK monitoring network from the 1960s until 2006. Particularly in the early years of the study, there is evidence that removed sites had a lower annual mean pollution reading than those which were added. Gelfand et al. (2012) showed the prediction effect of preferentially chosen ozone monitoring sites in California, whilst Diggle et al. (2010) and Dinsdale and Salibian-Barrera (2018) illustrated the effect that preferential sampling of lead concentration in Galicia may have on the resulting predictions. In this case, the investigation was whether or not stationary sampling locations were chosen preferentially for heavy metal biomonitoring in Galicia, or in other words, whether or not the locations at which lead concentration samples were obtained were dependent on speculation or knowledge of the lead concentrations at these locations. Pati et al. (2011) studied the effect of preferential selection of monitoring sites measuring ozone levels in Eastern U.S.A and more recently Conn et al. (2017) showed that preferential sampling in animal population surveys may cause large biases in the animal density estimates, giving an example using aerial survey counts of bearded seals in the Eastern Bering sea. A Bayesian design-based approach by da Silva Ferreira et al. (2015) assessed the impact of preferential sampling when choosing new sampling locations.

Evaluating the integral in (3.3) can be challenging, which makes its optimization a highly costly computational problem. Assuming the observed responses $\mathbf{Y}$ depend on the spatial process $S$ only through the values of the process at the locations $\mathbf{X}$ (in symbols: when $[\mathbf{Y} \mid S, \mathbf{X}]=[\mathbf{Y} \mid S(\mathbf{X})]$ ) the celebrated paper of Diggle et al. (2010) proposed an ingenious method using Monte Carlo (MC) samples from a carefully chosen conditional distribution of $S$, in order to improve the efficiency of each MC likelihood approximation.

However there are various issues in the MC approach of Diggle et al. (2010) which may impact both the parameter estimates of the model and the resulting spatial predictions. In this thesis we first discuss a possible way to address these problems in Chapter 4, but show that it still inherits similar issues as the one in Diggle et al. (2010). Furthermore, the efficiency of such an MC approach can still be very low when the model for $[\mathbf{X} \mid S]$ is moderately complex. We show that modern numerical methods such as those implemented in Template Model Builder (Kristensen et al., 2016) to optimize this type of likelihood function provide very good alternatives to MC approximations, particularly in a subtly complex problem such as preferential sampling. We show how these methods may be used for likelihood 
estimation and correct specification of the spatial prediction distribution in Sections 4.4 and 5.2 respectively.

Before this however, we must establish possible relationships between the sampling locations $\mathbf{X}$ and the random field $S$. For the purpose of this thesis we are essentially interested in four different systems by which the sampling locations $\mathbf{X}$ in Section 2.1 are modelled. These are

1. Non-preferentially sampled point processes.

2. Non-preferentially sampled movement processes.

3. Preferentially sampled point processes.

4. Preferentially sampled movement processes.

The point process cases are the instances that the locations at which we sample from are static in the spatial domain. An example of this would be a grid of monitoring stations as highlighted in Section 2.4. Movement process sampling is the case in which the locations at which we sample from are non-static, or in other words the locations are moving through the domain over time. For example, this could be the case in which sampling locations are obtained in discrete time from SDEs outlined in Section 2.5.

\subsection{Sampling from Point Processes}

We begin by discussing point process sampling processes. As mentioned previously, non-preferentially sampled locations are the cases in which $[\mathbf{X}]$ is independent of the random field of interest $S$, as opposed to preferentially sampled locations which are dependent on the field. The case of non-preferentially sampled and static locations (1. in our list) is a typical geostatistical problem which was described in Section 2.3. Standard maximum likelihood estimation (MLE) which assumes independence between sampling locations and the underlying surface under an assumed Gaussian model may be used to estimate model parameters and then methods such as kriging can be applied for spatial prediction.

In the case of point processes, defined in Section 2.4, all homogeneous and the most commonly used inhomogeneous processes are non-preferentially sampled. Homogeneous point processes are an obvious case, since the intensity function which determines the sampling probability is constant across all regions of the space, namely $\lambda(x)=c$ for all $x \in \mathcal{D}$ for some domain $\mathcal{D}$. Inhomogeneous point processes are where the intensity function depends 
on location. In this case, the process is still non-preferential so long as the intensity function is not a function of the field of interest $S$. Mathematically this is the case when $\lambda(x, S)=\lambda(x)$ for all $x \in \mathcal{D}$. In these instances we can assume independence between the field $S$ and the sampling locations and hence condition on the sampling locations in our analyses.

The most common example of preferential sampling found in literature is the preferential sampling of point processes (3. in our list). Examples of papers using this type model include Diggle et al. (2010); Gelfand et al. (2012); Pati et al. (2011). In all of these papers, inhomogeneous process models with intensity functions that depend on the field $S$ are used to model the sampling process. A slightly different technique was proposed by Zidek et al. (2014) for the case in which the number of preferentially located stations may change over time. Their work utilises the Horvitz-Thompson approach to compensate for bias due to preferential sampling and applies these methods to a case study assessing the levels of air pollution due to black smoke in the UK between 1970-1996. Arguably the work by Zidek et al. (2014) is as close to our preferentially sampled movement models as we have found, since although the sites are stationary, locations may change over time as new stations appear and others are closed.

\subsubsection{Example of Preferential Point Processes}

To give an example of a preferential sampling situation with static measurement locations we generate data following the model (2.1) with constant mean $\mu=4$ and nugget variance $\tau^{2}=0.10$. The process $S$ is Gaussian with a Matérn correlation structure as defined in (2.4) with scale (range) $\phi=0.05$, known shape parameter $\kappa=1$ and variance $\sigma^{2}=1.5$. We generate 500 fields which are simulated over a discrete $250 \times 250$ grid of equally-spaced points on the unit square.

On each field $\mathbf{S}_{j}$ for $j=1, \ldots, 500$ we simulate 4 data sets consisting of $n=100$ measurements each, $\mathbf{Y}=\left(Y_{1}, \ldots, Y_{n}\right)$, and corresponding sampling locations $\mathbf{X}=\left(\mathbf{X}_{1}, \ldots, \mathbf{X}_{n}\right)$ generated using the following location intensity function:

$$
\frac{\exp \{\beta \mathbf{S}(\mathbf{x})\}}{\int_{\mathcal{D}} \exp \{\beta \mathbf{S}(\mathbf{u})\} \mathrm{du}},
$$

where $\mathcal{D}$ is the spatial domain of the analyses, which in this case is the unit square. Each data set samples locations using different values of $\beta=$ $-1,0,1,2$. When $\beta=0$ we have uniform random sampling of the field, which is not a case of preferential sampling. When $\beta=-1$ the intensity function decreases at higher values of $\mathbf{S}$ and therefore sampling locations are 
more likely to be found in the lower valued regions, whilst the opposite is true when $\beta>0$.

In Figure 3.1 we show how the sampling locations change with varying $\beta$ values in the intensity function from $(3.4)$ on 1 of the 500 fields used in this simulation. The average sampled value of the field for various $\beta$ 's, denoted $\bar{y}$, reflects these sampling location patterns. The non-preferential sampling has the closest $\bar{y}$ in comparison to the true mean parameter $\mu=4$, whilst preferential sampling increased the absolute difference $|\bar{y}-\mu|$ as $|\beta|$ increased.

To assess the impact of this type of preferential sampling when using a naive inference approach, we compare parameter estimation and prediction across the varying $\beta$ data sets using standard MLE where the sampling locations are assumed independent of the field $S$. In other words we maximise the log-likelihood function from (2.6) and use these parameter estimates as plug-in values for ordinary kriging as defined in (2.11).

The parameter estimates are shown for all field parameters in Figure 3.2 , with true values used to generate the fields $\mathbf{S}_{j}$ marked in black. In the first panel the bias of the mean parameter estimates $\hat{\mu}$ induced by preferential sampling is clear to see. Under uniformly random sampling $(\beta=0)$ the estimates show no bias. However for the cases $\beta=-1,1$ there are a clear under and overestimation of the mean respectively. This is increased further in the case $\beta=2$ and is a direct consequence of the bias in the measurements $\mathbf{Y}$ used in the likelihood estimation.

The second and third panels in Figure 3.2 also show an increase in bias of the scale $(\hat{\phi})$ and marginal variance $\left(\hat{\sigma}^{2}\right)$ estimates under preferential sampling. In these cases it appears that as $|\beta|$ increases, the accuracy in the estimation decreases. This is due to the reduced coverage of the fields, since the preferential effect reduces the variability of the measurements and also clusters points closer to each other in areas of high preference for the corresponding $\beta$ value. Consequently the standard likelihood function does not use a representative sample of the field in terms of spatial correlation decay and variance. The nugget variance estimates $\left(\hat{\tau}^{2}\right)$ in the final panel do not appear to be affected by this preferential effect.

The corresponding prediction accuracy is assessed for ordinary kriging with plug-in parameters from Figure 3.2. The prediction error $(\mathrm{PE})$ and bias at location $\mathbf{X}_{0}$ are defined for the $j$ th field as

$$
\begin{aligned}
\operatorname{PE}_{j}\left(\mathbf{x}_{0}\right) & =\sqrt{\left(\hat{\mathbf{S}}_{j}\left(\mathbf{x}_{0}\right)-\mathbf{S}_{j}\left(\mathbf{x}_{0}\right)\right)^{2}}, \\
\operatorname{Bias}_{j}\left(\mathbf{x}_{0}\right) & =\hat{\mathbf{S}}_{j}\left(\mathbf{x}_{0}\right)-\mathbf{S}_{j}\left(\mathbf{x}_{0}\right),
\end{aligned}
$$




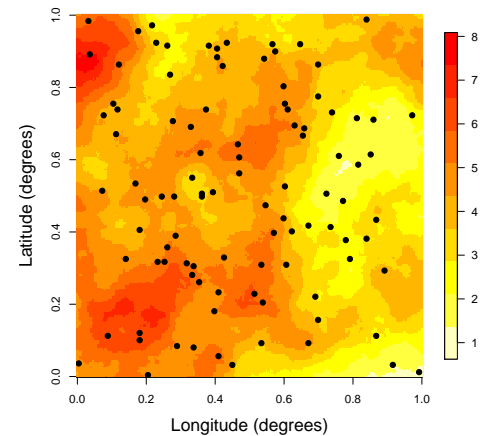

(a) $\beta=0, \bar{y}=4.09$

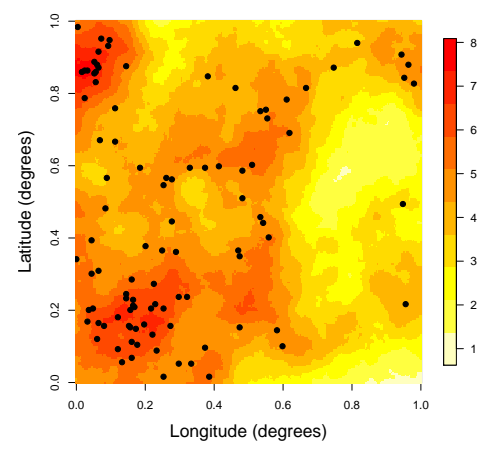

(c) $\beta=1, \bar{y}=5.42$

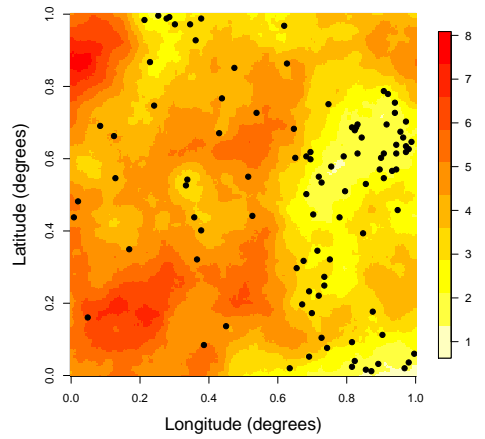

(b) $\beta=-1, \bar{y}=2.88$

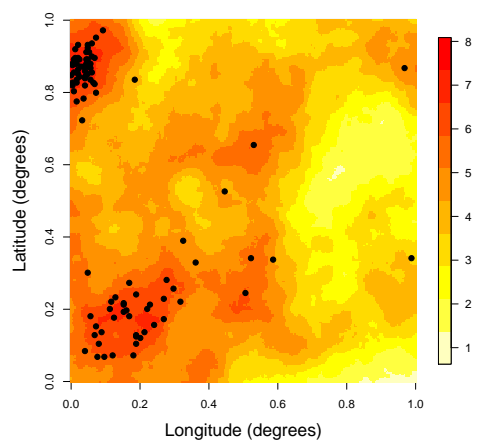

(d) $\beta=2, \bar{y}=6.51$

Figure 3.1: Example of sampling locations using the inhomogenous point process with intensity (3.4) with varying $\beta$ values on the same field $\mathbf{S}$. The $\bar{y}$ value is sample mean for that data set. 


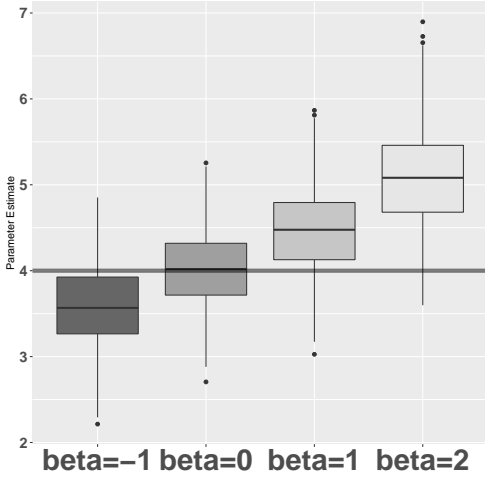

(a) Mean $(\mu)$

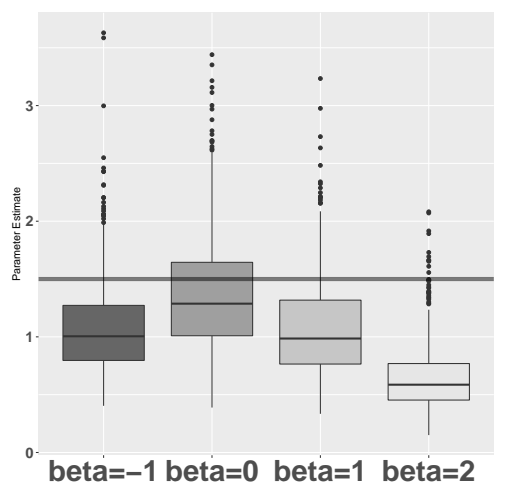

(c) Variance $\left(\sigma^{2}\right)$

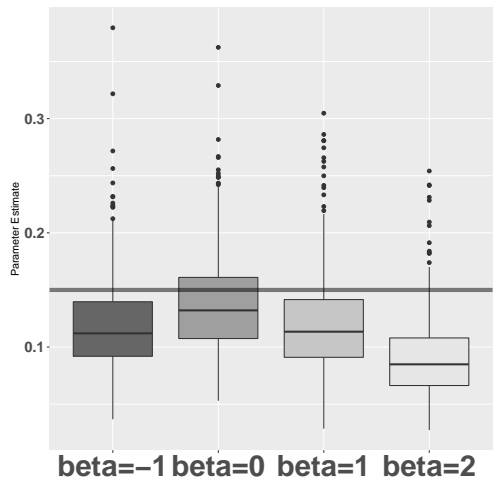

(b) Scale $(\phi)$

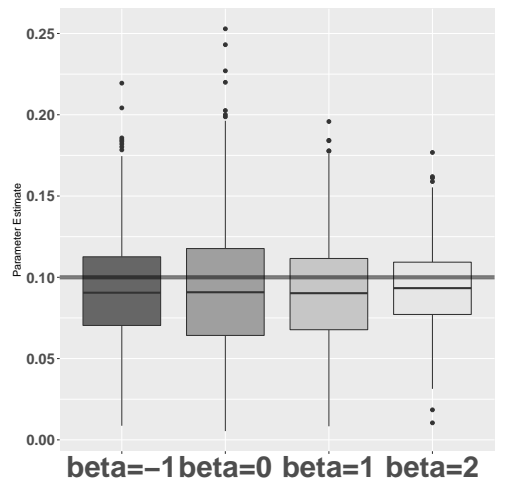

(d) Nugget variance $\left(\tau^{2}\right)$

Figure 3.2: Parameter estimates over 500 simulated data sets with varying $\beta$ parameters. True parameter values are marked as black horizontal lines. 
Table 3.1: $95 \%$ Confidence intervals for prediction error $(\mathrm{PE})$ and bias at prediction location $\mathbf{x}_{0}=(0.06,0.06)$.

\begin{tabular}{|c|c|c|c|c|}
\hline & $\beta=0$ & $\beta=-1$ & $\beta=1$ & $\beta=2$ \\
\hline $\mathrm{PE}$ & $(0.32,0.37)$ & $(0.47,0.56)$ & $(0.46,0.55)$ & $(0.98,1.14)$ \\
Bias & $(-0.04,0.04)$ & $(-0.40,-0.29)$ & $(0.29,0.40)$ & $(0.91,1.08)$ \\
\hline
\end{tabular}

where $\hat{\mathbf{S}}_{j}\left(\mathbf{x}_{0}\right)$ and $\mathbf{S}_{j}\left(\mathbf{x}_{0}\right)$ are the ordinary kriging prediction and true value of the field $\mathbf{S}_{j}$ at $\mathbf{x}_{0}$ respectively. The $95 \%$ confidence intervals for PE and Bias, taken as the mean plus and minus 2 standard errors over the 500 samples, are shown for $\mathbf{x}_{0}=(0.06,0.06)$ in Table 3.1. As $|\beta|$ increases, so does the prediction error showing that the kriging weights cannot fully compensate for the bias in the measurements $\mathbf{Y}$ and parameter estimates. The $95 \%$ confidence interval for the bias only includes 0 for $\beta=0$ and clearly the standard prediction methods cannot correct for the preferential effect.

This is a result of inaccurate parameter estimates, but also due to the kriging model ignoring the effect that sampling locations $\mathbf{X}$ have on the predictions. Since kriging assumes $\mathbf{S}$ and $\mathbf{X}$ are independent, the predictions do not account for the fact that the sampling locations might inform prediction. For example, understanding the relationship between $\mathbf{X}$ and $\mathbf{S}$ tells us not just information about areas of high sampling density, but also areas of sparse sampling density. In the case of $\beta=1$ this would be that sparsely sampled areas are likely to have low temperatures and hence prediction using extrapolation from sampled values will not capture the true change of the field. Even though kriging can slightly reduce the effect of preferential sampling by down weighing points sampled in close vicinity to each other, it does not fully eradicate the problem. To improve parameter estimation and prediction under preferential sampling we need to construct models that account for dependency between the measurements and the sampling locations. This can be used to reduce the bias caused by preferential sampling in the parameter estimates, but also to better inform the prediction model. We cover the predictive distribution in more detail in Section 5.2 .

\subsubsection{Drawbacks of the Point Process Approach}

For stationary sampling locations this thesis considers the use of a point processes with an intensity of the form in $(3.4)$, as is done regularly in literature. However, this approach may run into difficulties in some cases, which also brings into question the complication of identifying preferential sampling in certain scenarios. 
This issue is namely extremities in the preferential sampling structure that may result in $\beta$ being unidentifiable in (3.4). Take for example, a data set in which the preferential sampling is so extreme that only the very largest values of the field are sampled. Without any reference data in other, lower valued regions, identifying that the data has been preferentially sampled may be impossible. In this case, with no lower data values to identify the extent of the preferential sampling, $\beta$ would be unidentifiable. An extreme example of this would be to consider data only sampled at locations where the field $S$ is equal to some constant, say $c$. In this instance, we might expect the model to estimate $\hat{\sigma}^{2} \approx 0$ to predict an inaccurately flat surface, in which we may not be able to estimate $\beta$ consistently.

In summary, we will be assuming that there is at least a significant preferential effect throughout the stationary preferential sampling analysis in this thesis. However, extreme preferential sampling, as discussed above, may be an issue in possibly unidentified areas. Such data would need an alternative approach to first identify possible preferentiability and then to understand it accurately. This falls outside the scope of this thesis, but could be a valuable contribution to preferential sampling literature.

\subsection{Movement Processes}

For non-preferentially sampled locations obtained from a movement process (2. in our list) we consider the sampler to be a continuously moving process throughout the spatial domain, but the movement direction and speed is independent of $S$. This would be the case for discrete observations from a SDE with drift function $\boldsymbol{\mu}(\cdot)$ in (2.15) that does not depend on $S$. Although sampling locations depend on each other, which is not the case with point processes, the field $S$ still does not provide any information on the sampling locations and hence assuming them known is common practice. Preliminary investigations into this sampling scheme in the simulation from Section 8.4 suggest that standard maximum likelihood estimation on $Y$ and then ordinary kriging do an adequate job of predicting the partially observed field $S$.

Preferentially sampled movement models (4. in our list) is a major focus of this thesis and is introduced in Chapters $7 \sqrt{8}$. We only briefly discuss them here, since formulation of these models is rather more complex than the point process alternatives. This is the case in which sampling locations are obtained from continuously moving processes which are themselves moving in a preferential manner. This would occur for observations from an SDE 


\subsection{Movement Processes}

when $\boldsymbol{\mu}(\cdot)$ is a function of $S$, which would result in sampler movement depending on the field. There are multiple ways in which this might happen, many of which are discussed in Chapter 8. One example is the sampling of ocean temperatures by tagged marine mammals, where movement may depend on ocean temperature (Guinet et al., 2014; McIntyre et al., 2011). Models using preferential SDEs are developed in Section 8.3, with the aim of assessing the potential impact that preferential sampling may incur on ocean temperature prediction in the Southern Indian ocean and how we can correct for this. 


\section{Chapter 4}

\section{Maximum Likelihood Estimation Under Preferential Sampling}

This chapter introduces parameter estimation using maximum likelihood under preferential sampling. In Section 4.1 we show how to account for preferential sampling in the likelihood function, then review how this function may be approximated using the Monte Carlo approach of Diggle et al. (2010) in Section 4.2. We demonstrate how this approximation has derivation issues and recommend corrections in Section 4.3. We propose the use of Laplace approximations to the preferential likelihood function in Section 4.4, showing how we can utilise the R package Template Model Builder (TMB) (Kristensen et al., 2016) to create a flexible and efficient framework for preferential likelihood function evaluations. Finally, we assess the performance of TMB compared to other methods in simulated examples and a previously analysed data set in Sections 4.5 and 4.6 respectively.

\subsection{Specifying the Preferential Likelihood}

When using maximum likelihood estimation (MLE) under preferential sampling, one must take care in specification of the likelihood function, which in general does not follow standard forms discussed in Section 2.3. As introduced in Chapter 3, under preferential sampling we can no longer condition on the sampling locations due to their interaction with the latent field $S$ and must consider the preferential (full) likelihood

$$
L(\boldsymbol{\theta})=[\mathbf{X}, \mathbf{Y}]=\int[\mathbf{X}, \mathbf{Y}, S] \mathrm{d} S .
$$

Since we do not know the full joint density in the integrand from (4.1) we should consider the factorisation

$$
[\mathbf{X}, \mathbf{Y}, S]=[\mathbf{Y} \mid S, \mathbf{X}][\mathbf{X} \mid S][S] .
$$


We will assume that our data is of the form described in Section 2.1, namely that the $n$-observations $\mathbf{Y}=\left(Y_{1}, \ldots, Y_{n}\right)$ are sampled at $\mathbf{X}=$ $\left(\mathbf{X}_{1}, \ldots, \mathbf{X}_{n}\right)$ following from $(2.1)$ and that $S$ is a Gaussian Process with some spatial covariance structure. It is also necessary to discretise $S$ into a vector $\mathbf{S}$, consisting of $S$ at $N$ locations, where preferably $N$ is large to closely approximate the continuous equivalent. Once discretised, if we assume that $\mathbf{S}$ has some mean trend $\mathbf{D}_{N} \boldsymbol{\zeta}$ where $\mathbf{D}_{N}$ is an $N \times p$ matrix of covariates and $\boldsymbol{\zeta}$ a $p$-dimensional vector of regression coefficients, we have that

$$
\mathbf{S} \sim N_{N}\left(\mathbf{D}_{N} \boldsymbol{\zeta}, \Sigma\right)
$$

for some covariance matrix $\Sigma$. Furthermore we can specify

$$
\begin{aligned}
\mathbf{Y} \mid \mathbf{S}, \mathbf{X} & \sim N_{n}\left(\mathbf{S}_{0}, \tau^{2} I_{n}\right), \\
{[\mathbf{X} \mid \mathbf{S}] } & =f(\mathbf{S}),
\end{aligned}
$$

where $f(\cdot)$ is some probability density function depending on $\mathbf{S}$ that defines the sampling location structure and $\mathbf{S}_{0}$ the value of $\mathbf{S}$ at the $n$ sampling locations $\mathbf{X}$. Here we are assuming the locations of our discretised form of $S$ contains all the sampling locations, so $N \geq n$.

\subsection{Monte Carlo Approximation to the Preferential Likelihood}

A simple Monte Carlo approximation to the integral in (4.1) is therefore

$$
\hat{L}(\boldsymbol{\theta})=\frac{1}{m} \sum_{j=1}^{m}\left[\mathbf{Y} \mid \mathbf{S}_{j}, \mathbf{X}\right]\left[\mathbf{X} \mid \mathbf{S}_{j}\right]\left[\mathbf{S}_{j}\right],
$$

where $\mathbf{S}_{j}$ are randomly drawn from the distribution of $\mathbf{S}$ with parameters $\boldsymbol{\theta}$. Generally, this method will require an extremely large number of samples, $m$, because many of the realizations $\mathbf{S}_{j}$ may not be compatible with the observed measurements in $\mathbf{Y}$ and hence a large number of terms from $\left[\mathbf{Y} \mid \mathbf{S}_{j}, \mathbf{X}\right]$ in (4.4) will be equal to 0 .

To avoid this problem Diggle et al. (2010) proposed an alternative representation of the likelihood function that relies on importance sampling to reduce the $\mathrm{MC}$ variability

$$
L(\boldsymbol{\theta})=\int[\mathbf{X}, \mathbf{Y}, S] \mathrm{d} S=\int[\mathbf{Y} \mid S, \mathbf{X}][\mathbf{X} \mid S] \frac{[S \mid \mathbf{Y}]}{[S \mid \mathbf{Y}]}[S] \mathrm{d} S
$$


Diggle et al. (2010) rewrites a discretised version of this integral as

$$
\begin{aligned}
L(\boldsymbol{\theta}) & \approx \int[\mathbf{Y} \mid \mathbf{S}, \mathbf{X}][\mathbf{X} \mid \mathbf{S}] \frac{[\mathbf{S} \mid \mathbf{Y}]}{[\mathbf{S} \mid \mathbf{Y}]}[\mathbf{S}] \mathrm{d} \mathbf{S} \\
& =\int\left[\mathbf{Y} \mid \mathbf{S}_{0}\right][\mathbf{X} \mid \mathbf{S}] \frac{[\mathbf{S} \mid \mathbf{Y}]}{[\mathbf{S} \mid \mathbf{Y}]}\left[\mathbf{S}_{0}\right]\left[\mathbf{S}_{1} \mid \mathbf{S}_{0}\right] \mathrm{d} \mathbf{S} \\
& =\int\left[\mathbf{Y} \mid \mathbf{S}_{0}\right][\mathbf{X} \mid \mathbf{S}] \frac{[\mathbf{S} \mid \mathbf{Y}]}{\left[\mathbf{S}_{0} \mid \mathbf{Y}\right]\left[\mathbf{S}_{1} \mid \mathbf{S}_{0}, \mathbf{Y}\right]}\left[\mathbf{S}_{0}\right]\left[\mathbf{S}_{1} \mid \mathbf{S}_{0}\right] \mathrm{d} \mathbf{S}, \\
& =\int\left[\mathbf{Y} \mid \mathbf{S}_{0}\right][\mathbf{X} \mid \mathbf{S}] \frac{[\mathbf{S} \mid \mathbf{Y}]}{\left[\mathbf{S}_{0} \mid \mathbf{Y}\right]\left[\mathbf{S}_{1} \mid \mathbf{S}_{0}\right]}\left[\mathbf{S}_{0}\right]\left[\mathbf{S}_{1} \mid \mathbf{S}_{0}\right] \mathrm{d} \mathbf{S}, \\
& =\int[\mathbf{X} \mid \mathbf{S}] \frac{\left[\mathbf{Y} \mid \mathbf{S}_{0}\right]}{\left[\mathbf{S}_{0} \mid \mathbf{Y}\right]}\left[\mathbf{S}_{0}\right][\mathbf{S} \mid \mathbf{Y}] \mathrm{d} \mathbf{S} \\
& \left.=\mathbb{E}_{\mathbf{S} \mid \mathbf{Y}}[\mathbf{X} \mid \mathbf{S}] \frac{\left[\mathbf{Y} \mid \mathbf{S}_{0}\right]}{\left[\mathbf{S}_{0} \mid \mathbf{Y}\right]}\left[\mathbf{S}_{0}\right]\right]
\end{aligned}
$$

where $\mathbf{S}=\left\{\mathbf{S}_{0}, \mathbf{S}_{1}\right\}$ denotes a discretised version of the continuous process $S$ on a 2-dimensional lattice, $\mathbf{S}_{0}$ contains the values of $\mathbf{S}$ at the observed locations $\mathbf{X}$, and the remaining lattice points are in $\mathbf{S}_{1}$. This representation suggests the following Monte Carlo estimate

$$
L(\boldsymbol{\theta}) \approx \frac{1}{m} \sum_{j=1}^{m}\left[\mathbf{X} \mid \mathbf{S}_{j}\right] \frac{\left[\mathbf{Y} \mid \mathbf{S}_{0 j}\right]}{\left[\mathbf{S}_{0 j} \mid \mathbf{Y}\right]}\left[\mathbf{S}_{0 j}\right],
$$

where $\mathbf{S}_{j}=\left\{\mathbf{S}_{0 j}, \mathbf{S}_{1 j}\right\}$ are simulated independent realizations of $\mathbf{S}$ conditional on $\mathbf{Y}$ on the discrete 2-dimensional lattice, with $\mathbf{S}_{0 j}$ containing the values of $\mathbf{S}_{j}$ at the data locations and $\mathbf{S}_{1 j}$ those at the remaining lattice points.

The goal of the derivation in (4.6) is to obtain a more efficient Monte Carlo likelihood estimator by simulating realisations of $\mathbf{S} \mid \mathbf{Y}$ that are expected to be more compatible with the observations $\mathbf{Y}$ than realisations of $\mathbf{S}$ from (4.4).

Note that the form of the likelihood approximation in $(4.6)$ was derived by assuming that $[\mathbf{Y} \mid \mathbf{S}, \mathbf{X}]=\left[\mathbf{Y} \mid \mathbf{S}_{0}, \mathbf{X}\right]$. Therefore, for this model the dependency of the observed responses $\mathbf{Y}$ on the random field $\mathbf{S}$ happens only at the sampling location of each measurement. In other words there are no "neighbour" or "upstream" effects of $\mathbf{S}$ on the response variable. In this case we have $[\mathbf{Y} \mid \mathbf{S}, \mathbf{X}]=[\mathbf{Y} \mid \mathbf{S}(\mathbf{X})]$. This holds for most models discussed in the geostatistical literature.

However, there are two key issues surrounding the Monte Carlo likelihood estimate in (4.6) that will be discussed in more detail. We outline them below in what we believe are levels of increasing importance. 


\subsection{Monte Carlo Solution Problems and Correction}

\subsubsection{Implicit Conditioning of $\mathrm{X}$}

The first issue is the implicit conditioning on $\mathbf{X}$ in (4.6) above, which if appropriately accounted for reveals issues within the calculation of the integrand in that equation and with the specification of the MC sampling distribution $\mathbf{S} \mid \mathbf{Y}$.

First consider $\mathbf{S}_{0}$ from $(4.6)$, which is defined as the values of the discretised field $\mathbf{S}$ at the known sampling locations. If we explicitly include in the notation the necessary conditioning on $\mathbf{X}$ we can more clearly write the last terms of the expectation in 4.6$)$ as

$$
\left[\mathbf{Y} \mid \mathbf{S}_{0}\right] \frac{\left[\mathbf{S}_{0}\right]}{\left[\mathbf{S}_{0} \mid \mathbf{Y}\right]}=\left[\mathbf{Y} \mid \mathbf{S}_{0}, \mathbf{X}\right] \frac{\left[\mathbf{S}_{0} \mid \mathbf{X}\right]}{\left[\mathbf{S}_{0} \mid \mathbf{Y}, \mathbf{X}\right]}
$$

Then it becomes clear that

$$
\left[\mathbf{Y} \mid \mathbf{S}_{0}\right] \frac{\left[\mathbf{S}_{0}\right]}{\left[\mathbf{S}_{0} \mid \mathbf{Y}\right]}=\frac{\left[\mathbf{S}_{0}, \mathbf{Y} \mid \mathbf{X}\right]}{\left[\mathbf{S}_{0} \mid \mathbf{Y}, \mathbf{X}\right]}=[\mathbf{Y} \mid \mathbf{X}]
$$

Therefore the integrand in $(4.6)$ equals $[\mathbf{X} \mid \mathbf{S}][\mathbf{Y} \mid \mathbf{X}]$, and its corresponding $\mathrm{MC}$ estimator may not be approximating the desired likelihood function.

This error occurs at the heart of the derivation of $(4.6)$ which uses the factorisations $[\mathbf{S}]=\left[\mathbf{S}_{0}\right]\left[\mathbf{S}_{1} \mid \mathbf{S}_{0}\right]$ and $[\mathbf{S} \mid \mathbf{Y}]=\left[\mathbf{S}_{0} \mid \mathbf{Y}\right]\left[\mathbf{S}_{1} \mid \mathbf{S}_{0}\right]$. When they hold, we can simplify some terms in the importance sampler $[\mathbf{S} \mid \mathbf{Y}]$ :

$$
\begin{aligned}
L(\boldsymbol{\theta}) & \approx \int[\mathbf{Y} \mid \mathbf{S}, \mathbf{X}][\mathbf{X} \mid \mathbf{S}] \frac{[\mathbf{S} \mid \mathbf{Y}]}{[\mathbf{S} \mid \mathbf{Y}]}[\mathbf{S}] \mathrm{d} \mathbf{S}, \\
& =\int[\mathbf{Y} \mid \mathbf{S}, \mathbf{X}][\mathbf{X} \mid \mathbf{S}] \frac{[\mathbf{S} \mid \mathbf{Y}]}{\left[\mathbf{S}_{0} \mid \mathbf{Y}\right]\left[\mathbf{S}_{1} \mid \mathbf{S}_{0}\right]}\left[\mathbf{S}_{0}\right]\left[\mathbf{S}_{1} \mid \mathbf{S}_{0}\right] \mathrm{d} \mathbf{S}, \\
& =\int[\mathbf{Y} \mid \mathbf{S}, \mathbf{X}][\mathbf{X} \mid \mathbf{S}] \frac{[\mathbf{S} \mid \mathbf{Y}]}{\left[\mathbf{S}_{0} \mid \mathbf{Y}\right]}\left[\mathbf{S}_{0}\right] \mathrm{d} \mathbf{S} .
\end{aligned}
$$

If we explicitly include the conditioning on $\mathbf{X}$ for the importance sampling distribution above, and use $[\mathbf{S} \mid \mathbf{Y}, \mathbf{X}]$, we can still factorise it in terms of $\mathbf{S}_{0}$ and $\mathbf{S}_{1}:[\mathbf{S} \mid \mathbf{Y}, \mathbf{X}]=\left[\mathbf{S}_{0} \mid \mathbf{Y}, \mathbf{X}\right]\left[\mathbf{S}_{1} \mid \mathbf{S}_{0}, \mathbf{X}\right]$. However we cannot factorise the term $[\mathbf{S}]$ in a way that allows the cancellation of $\left[\mathbf{S}_{1} \mid \mathbf{S}_{0}, \mathbf{X}\right]$. Although $[\mathbf{S} \mid \mathbf{X}]=\left[\mathbf{S}_{0} \mid \mathbf{X}\right]\left[\mathbf{S}_{1} \mid \mathbf{S}_{0}, \mathbf{X}\right]$ this does not help to simplify the term $[\mathbf{S}]$ in the integrand above. Hence, one cannot use the same arguments as in Diggle et al. $(2010)$ since $\left[\mathbf{S}_{1} \mid \mathbf{S}_{0}, \mathbf{X}\right] \neq\left[\mathbf{S}_{1} \mid \mathbf{S}_{0}\right]$ and in fact both $\left[\mathbf{S}_{0}\right]$ and $\left[\mathbf{S}_{1} \mid \mathbf{S}_{0}\right]$ appear intractable under preferential sampling. 


\subsection{Monte Carlo Solution Problems and Correction}

Another issue that arises when ignoring the conditioning on $\mathbf{X}$ is the specification of the Monte Carlo sampling distribution $\mathbf{S} \mid \mathbf{Y}$. Diggle et al. (2010) used a constant mean equivalent to

$$
\begin{aligned}
N_{N}\left(\mathbf{D}_{N} \boldsymbol{\zeta}+\Sigma_{N n}\left(\Sigma_{n}+\tau^{2} I_{n}\right)^{-1}\right. & \left(\mathbf{y}-\mathbf{D}_{n} \boldsymbol{\zeta}\right) \\
& \left.\Sigma_{N}-\Sigma_{N n}\left(\Sigma_{n}+\tau^{2} I_{n}\right)^{-1} \Sigma_{n N}\right)
\end{aligned}
$$

where $\Sigma_{N}=\operatorname{Cov}(\mathbf{S})$ is the $N \times N$ covariance matrix of $\mathbf{S}, \mathbf{D}_{n}=\mathbf{C D}_{N}$, $\Sigma_{n}=\mathbf{C} \Sigma_{N} \mathbf{C}^{\top}, \Sigma_{N n}=\Sigma_{N} \mathbf{C}^{T}=\Sigma_{n N}^{\top}$, and $\boldsymbol{C}$ is an $n \times N$ matrix whose $i$ th row contains $(N-1) 0$ 's and a single 1 indicating the position of the $i$ th observed location within the lattice. If we were to assume a constant mean trend for the field $\mathbf{S}$, then we would replace $\mathbf{D}_{N} \boldsymbol{\zeta}$ and $\mathbf{D}_{n} \boldsymbol{\zeta}$ with $\mu \mathbf{1}_{N}$ and $\mu \mathbf{1}_{n}$ respectively, where $\mu$ is our trend parameter and $\mathbf{1}_{p}$ a $p$-dimensional vector of 1's.

However, the distribution in (4.8) is in fact implicitly conditioning on the measurement locations $\mathbf{X}$, something that was overlooked in Diggle et al. (2010). The true distribution $\mathbf{S} \mid \mathbf{Y}$ is given by

$$
\begin{aligned}
{[\mathbf{S} \mid \mathbf{Y}] } & =\frac{[\mathbf{S}, \mathbf{Y}]}{[\mathbf{Y}]} \\
& =\int \frac{[\mathbf{S}, \mathbf{X}, \mathbf{Y}]}{[\mathbf{Y}]} \mathrm{d} \mathbf{X} \\
& =\int[\mathbf{S} \mid \mathbf{X}, \mathbf{Y}][\mathbf{X} \mid \mathbf{Y}] \mathrm{d} \mathbf{X} \\
& =\mathbb{E}_{\mathbf{X} \mid \mathbf{Y}}[[\mathbf{S} \mid \mathbf{X}, \mathbf{Y}]]
\end{aligned}
$$

This last equation suggests that that the method in Diggle et al. (2010) was simply approximating $[\mathbf{S} \mid \mathbf{Y}]$ by plugging in the actual realisation of the measurement locations $\mathbf{X}$ rather than taking the expectation with respect to $[\mathbf{X} \mid \mathbf{Y}]$, which appears to be intractable. However, in the next paragraph we show that the distribution in $(4.8)$ may provide such an approximation only under a non-preferential model.

\subsection{2 $\quad$ Specification of $[\mathrm{S} \mid \mathrm{X}, \mathrm{Y}]$}

Although the proposed distribution in (4.8) may be considered to be an approximation to the correct one in (4.9), it is important to note that, in fact, it does not take into account the dependency between $\mathbf{S}$ and $\mathbf{X}$, and 


\subsection{Monte Carlo Solution Problems and Correction}

instead it is that of $[\mathbf{S} \mid \mathbf{X}, \mathbf{Y}]$ under a non-preferential model. Indeed, when no preferential sampling is present we have

$$
[\mathbf{Y} \mid \mathbf{X}]=\mathbb{E}_{\mathbf{S} \mid \mathbf{X}}[[\mathbf{Y} \mid \mathbf{S}, \mathbf{X}]]=\mathbb{E}_{\mathbf{S}}[[\mathbf{Y} \mid \mathbf{S}, \mathbf{X}]] \sim N_{n}\left(\mathbf{D}_{n} \boldsymbol{\zeta}, \Sigma_{n}+\tau^{2} I_{n}\right),
$$

since $[\mathbf{S} \mid \mathbf{X}]=[\mathbf{S}] \sim N_{N}\left(\mathbf{D}_{N} \boldsymbol{\zeta}, \Sigma_{N}\right)$. This result is then used to derive the form of $[\mathbf{S} \mid \mathbf{Y}, \mathbf{X}]$ found in $(4.8)$ using properties of the conditional multivariate normal distribution since we know both $[\mathbf{S} \mid \mathbf{X}]$ and $[\mathbf{Y} \mid \mathbf{X}]$. Further details on this derivation are given in (5.4). However, this argument cannot be used under preferential sampling when $[\mathbf{S} \mid \mathbf{X}] \neq[\mathbf{S}]$. In this case the distribution of $\mathbf{S} \mid \mathbf{Y}, \mathbf{X}$ is deceptively complex, and we believe it to be intractable, together with that of $\mathbf{S} \mid \mathbf{X}$ and $\mathbf{Y} \mid \mathbf{X}$.

This misspecification has implications in both the MC likelihood methods and also the corresponding spatial predictions. Aside from causing Monte Carlo simulations to sample from an incorrect distribution, ignoring the preferential effect when deriving $[\mathbf{S} \mid \mathbf{Y}, \mathbf{X}]$ also results in naive spatial predictions that do not take into account useful information provided by the preferential model, even with corrected field parameter estimates. This is discussed in more detail in Section 5.4.

\subsubsection{An Alternative Monte Carlo Solution}

Notwithstanding the issues highlighted in Sections 4.3.1 and 4.3.2, the idea of finding an expression for the likelihood that is amenable to MC approximation can be used to obtain:

$$
\begin{aligned}
{[\mathbf{X}, \mathbf{Y}] } & =\int[\mathbf{X}, \mathbf{Y}, \mathbf{S}] \mathrm{d} \mathbf{S}, \\
& =\int[\mathbf{Y} \mid \mathbf{S}, \mathbf{X}][\mathbf{X} \mid \mathbf{S}][\mathbf{S}] \frac{[\mathbf{S} \mid \mathbf{Y}, \mathbf{X}]}{[\mathbf{S} \mid \mathbf{Y}, \mathbf{X}]} \mathrm{d} \mathbf{S}, \\
& =\mathbb{E}_{\mathbf{S} \mid \mathbf{Y}, \mathbf{X}}\left([\mathbf{Y} \mid \mathbf{S}, \mathbf{X}] \frac{[\mathbf{X} \mid \mathbf{S}][\mathbf{S}]}{[\mathbf{S} \mid \mathbf{Y}, \mathbf{X}]}\right)
\end{aligned}
$$

Utilising a Monte Carlo estimator for (4.11) above amounts to using an "importance sampler" to approximate (4.10). Since in a preferential sampling context the distribution of $\mathbf{S} \mid \mathbf{Y}, \mathbf{X}$ is generally intractable, we cannot use this approach exactly as prescribed above. However, this argument suggests that if we could sample from a distribution close to it, then the above MC approximation might yield a relatively efficient estimator. In fact, if one were to sample from $[\mathbf{S} \mid \mathbf{Y}, \mathbf{X}]$ and use (4.11), then the integrand would be 
constant (resulting in no Monte Carlo variability):

$$
[\mathbf{Y} \mid \mathbf{S}, \mathbf{X}] \frac{[\mathbf{X} \mid \mathbf{S}][\mathbf{S}]}{[\mathbf{S} \mid \mathbf{Y}, \mathbf{X}]}=[\mathbf{Y} \mid \mathbf{S}, \mathbf{X}] \frac{[\mathbf{X}, \mathbf{S}]}{[\mathbf{S} \mid \mathbf{Y}, \mathbf{X}]}=\frac{[\mathbf{Y}, \mathbf{S}, \mathbf{X}]}{[\mathbf{S} \mid \mathbf{Y}, \mathbf{X}]}=[\mathbf{X}, \mathbf{Y}]
$$

In light of these observations, one could re-visit the approach in Diggle et al. (2010) using (4.8) instead of $[\mathbf{S} \mid \mathbf{Y}, \mathbf{X}]$ in (4.11), and without simplifying the integrand. However, as noted before, this approximation can only be expected to work well when there is no preferential sampling. Our numerical experiments in Section 4.5 suggest that when preferential sampling is present such an approximation may not be very good.

\subsubsection{Other Modelling Approaches}

There are also some alternative approaches utilising MC simulation that may be considered. One example could be the use of the multilevel MC approach by Giles (2013). This method is similar to the control variate approach (Glasserman, 2013) which constructs an unbiased estimator for the expectation of a variable by utilising a highly correlated variable to the one we wish to evaluate, which has a known expectation. The multilevel approach expands this further by using a sequence of approximations to the variable we wish to find the expectation of. This sequence approximates our target with increasing accuracy, but at a greater computational cost. However, one can utilise a greater number of MC simulations from the more computationally efficient but less accurate variables in comparison to the less efficient but more accurate ones. There may be potential to use this approach by constructing the sequence of variables via an increasing spatial grid resolution, similar to the multilevel approach for SPDEs mentioned by Giles (2015).

One may also consider a Bayesian paradigm, for example by utilising Markov Chain Monte Carlo (MCMC) (Metropolis et al., 1953). This approach has many attractive features, such as accounting for non-Gaussianity of parameter uncertainty (Minasny et al., 2011) and combining the practices of estimation and prediction into a single procedure (Diggle and Ribeiro, 2007). This tends to result in more conservative predictions of $S$, since these approaches account for the uncertainty in the model parameter estimates $(\boldsymbol{\theta})$ (Diggle et al., 1998). In the likelihood approach we have considered so far, described in Section 2.3, the model estimates are simply used as "plugged-in" parameters for the predictive distribution with their uncertainty ignored. An example of how to construct an MCMC algorithm for preferential sampling correction is given by da Silva Ferreira et al. (2015). 
The biggest drawback of using a MCMC approach is the computational challenge associated with the high dimensional simulation over $\mathbf{S}$. This is similar to the issues mentioned in the MC approach described so far in this chapter, but is exacerbated by highly dependent samples, which often requires thinning of the MC chain output (Diggle et al., 2010). For the application of this thesis, which aims to apply preferential sampling correction to more complex sampling structures, we have decided not to use this approach. Utilising MCMC is certainly a viable option, but would need some development to account for preferential sampling of moving objects, which is the final aim of our thesis and introduced in Chapter 8 .

\subsection{A Laplace Approximation to the Likelihood}

An alternative method to the $\mathrm{MC}$ simulation technique discussed above is to use the R package TMB, short for Template Model Builder (Kristensen et al., 2016). This package utilises automatic differentiation (AD) (Griewank and Walther, 2008) of a Laplace Approximation to the marginal likelihood to efficiently maximise the preferential likelihood with respect to the parameters of interest. This is another method of evaluating the large dimensional integral approximated by MC simulation in Section 4.2, We first describe the Laplace approximation to the likelihood function in Section 4.4.1 and follow in Section 4.4 .2 with details on the AD approach used to obtain a derivative function of the likelihood.

\subsubsection{The Laplace Approximation}

We begin by writing the likelihood function for $\boldsymbol{\theta}$ as

$$
\begin{aligned}
L(\boldsymbol{\theta}) & =[\mathbf{X}, \mathbf{Y}], \\
& =\int[\mathbf{X}, \mathbf{Y}, \mathbf{S}] \mathrm{d} \mathbf{S}, \\
& =\int \exp (-f(\mathbf{S}, \boldsymbol{\theta})) \mathrm{d} \mathbf{S},
\end{aligned}
$$

for some function $f$, which in our context would be

$$
f(\mathbf{S}, \boldsymbol{\theta})=-\log ([\mathbf{X}, \mathbf{Y}, \mathbf{S}]) .
$$

If we define

$$
\hat{\mathbf{S}}(\boldsymbol{\theta})=\underset{\mathbf{S}}{\arg \min } f(\mathbf{S}, \boldsymbol{\theta}),
$$


as the minimiser of $f(\mathbf{S}, \boldsymbol{\theta})$ with respect to the random field $\mathbf{S}$, then the Laplace approximation for the marginal likelihood $L(\boldsymbol{\theta})$ utilises the Taylor series expansion of $f(\mathbf{S}, \boldsymbol{\theta})$ about $\hat{\mathbf{S}}(\boldsymbol{\theta})$,

$$
\begin{aligned}
& f(\mathbf{S}, \boldsymbol{\theta})=f(\hat{\mathbf{S}}, \boldsymbol{\theta})+\frac{\partial}{\partial \mathbf{S}} f(\hat{\mathbf{S}}, \boldsymbol{\theta})(\mathbf{S}-\hat{\mathbf{S}})+ \\
& \frac{1}{2}(\mathbf{S}-\hat{\mathbf{S}})^{T} \frac{\partial^{2}}{\partial \mathbf{S}^{2}} f(\hat{\mathbf{S}}, \boldsymbol{\theta})(\mathbf{S}-\hat{\mathbf{S}})+\cdots
\end{aligned}
$$

where $\hat{\mathbf{S}}=\hat{\mathbf{S}}(\boldsymbol{\theta})$ is used here for simpler notation.

Since

$$
\left.\frac{\partial}{\partial \mathbf{S}} f(\mathbf{S}, \boldsymbol{\theta})\right|_{\mathbf{S}=\hat{\mathbf{S}}(\boldsymbol{\theta})}=0,
$$

we can ignore the first order term in $(4.15)$ and approximate $f(\mathbf{S}, \boldsymbol{\theta})$ via the second order element as

$$
f(\mathbf{S}, \boldsymbol{\theta}) \approx f(\hat{\mathbf{S}}, \boldsymbol{\theta})+\frac{1}{2}(\mathbf{S}-\hat{\mathbf{S}})^{T} \frac{\partial^{2}}{\partial \mathbf{S}^{2}} f(\hat{\mathbf{S}}, \boldsymbol{\theta})(\mathbf{S}-\hat{\mathbf{S}}) .
$$

Combining (4.12) and (4.17) we get the approximation

$$
\begin{aligned}
L(\boldsymbol{\theta}) & \approx \int \exp \left\{-f(\hat{\mathbf{S}}, \boldsymbol{\theta})-\frac{1}{2}(\mathbf{S}-\hat{\mathbf{S}})^{T} H(\boldsymbol{\theta})(\mathbf{S}-\hat{\mathbf{S}})\right\} \mathrm{d} \mathbf{S}, \\
& =\exp \{-f(\hat{\mathbf{S}}, \boldsymbol{\theta})\} \int \exp \left\{-\frac{1}{2}(\mathbf{S}-\hat{\mathbf{S}})^{T} H(\boldsymbol{\theta})(\mathbf{S}-\hat{\mathbf{S}})\right\} \mathrm{d} \mathbf{S}, \\
& =\exp \{-f(\hat{\mathbf{S}}, \boldsymbol{\theta})\}(2 \pi)^{\frac{N}{2}} \operatorname{det}(H(\boldsymbol{\theta}))^{-\frac{1}{2}}
\end{aligned}
$$

where $N$ is the dimension of $\mathbf{S}$ and $H(\boldsymbol{\theta})$ is the Hessian with respect to $\mathbf{S}$ evaluated at $\hat{\mathbf{S}}(\boldsymbol{\theta})$

$$
H(\boldsymbol{\theta})=\left.\frac{\partial^{2}}{\partial \mathbf{S}^{2}} f(\mathbf{S}, \boldsymbol{\theta})\right|_{\mathbf{S}=\hat{\mathbf{S}}(\boldsymbol{\theta})} .
$$

The final equality in (4.18) uses the definition of the multivariate normal density function and completes the derivation for our Laplace approximation to the likelihood $L(\boldsymbol{\theta})$, formally defined

$$
L^{1}(\boldsymbol{\theta})=(2 \pi)^{\frac{N}{2}} \operatorname{det}(H(\boldsymbol{\theta}))^{-\frac{1}{2}} \exp \{-f(\hat{\mathbf{S}}(\boldsymbol{\theta}), \boldsymbol{\theta})\} .
$$

TMB minimises $-l^{1}(\boldsymbol{\theta})=-\log \left(L^{1}(\boldsymbol{\theta})\right)$ efficiently by using automatic differentiation (introduced in Section 4.4.2) to acquire derivatives of $-l^{1}(\boldsymbol{\theta})$ with respect to $\boldsymbol{\theta}$. 
This technique overcomes many of the limitations of the MC methods described previously, namely: computational inefficiency for complex preferential sampling distributions and challenges introduced by the presence of MC variability in each function evaluation. We also found TMB to be very flexible, especially when defining relatively complex model structures. This is because in TMB the user has total control over the form of their function $f(\mathbf{S}, \boldsymbol{\theta})$, which is written in a $\mathrm{C}++$ script. The package integrates out the random effects and also computes the likelihood derivatives. In the case of preferential sampling, we simply have to define the joint negative log-likelihood

$$
f(\mathbf{S}, \boldsymbol{\theta})=-\log ([\mathbf{Y} \mid \mathbf{S}, \mathbf{X}][\mathbf{X} \mid \mathbf{S}][\mathbf{S}]),
$$

and allow TMB to integrate out the latent field $\mathbf{S}$ to evaluate the preferential likelihood function approximately.

\subsubsection{Automatic Differentiation}

For reference we give a brief overview of automatic differentiation (AD), the method utilised by TMB for likelihood gradient calculation. AD refers to a group of techniques that use the chain rule to compute numerical evaluation of function derivatives in the form of a computer algorithm (Fournier et al., 2012). These methods offer an alternative to derivative calculation based on finite differences which may be unstable, or the use analytical expressions for the derivative which are oftentimes hard to derive for complex functions.

AD comes in two flavours, forward and reverse accumulation, which work their way through the chain rule in different directions. Say we can write our scalar objective function $g(\boldsymbol{\theta})$ where $\boldsymbol{\theta}=\left(\theta_{1}, \ldots \theta_{p}\right)$ as

$$
g(\boldsymbol{\theta})=t_{n}\left(t_{n-1}\left(\cdots\left(t_{1}(\boldsymbol{\theta})\right) \cdots\right)\right),
$$

then to calculate the derivative with respect to $\theta_{i}$ for $i=1, \ldots, p$, forward accumulation uses the chain rule

$$
\frac{\partial g}{\partial \theta_{i}}=\frac{\partial g}{\partial t_{n}} \frac{\partial t_{n}}{\partial \theta_{i}}=\frac{\partial g}{\partial t_{n}}\left(\frac{\partial t_{n}}{\partial t_{n-1}} \frac{\partial t_{n-1}}{\partial \theta_{i}}\right)=\cdots=\frac{\partial g}{\partial t_{n}}\left(\frac{\partial t_{n}}{\partial t_{n-1}} \cdots \frac{\partial t_{2}}{\partial t_{1}} \frac{\partial t_{1}}{\partial \theta_{i}}\right) .
$$

In forward accumulation, the computational cost of evaluating the derivative with respect to a single parameter $\theta_{i}$ is less than four times that of evaluating the objective function itself (Skaug and Fournier, 2006). Unfortunately as we increase the number of parameters $p$, the cost of the full derivative with respect to $\boldsymbol{\theta}$ also grows due to the need of repeatedly calculating each derivative. 
This is not the case with the reverse accumulation method. In this case the derivation goes backwards through the chain rule

$$
\frac{\partial g}{\partial \theta_{i}}=\frac{\partial g}{\partial t_{1}} \frac{\partial t_{1}}{\partial \theta_{i}}=\left(\frac{\partial g}{\partial t_{2}} \frac{\partial t_{2}}{\partial t_{1}}\right) \frac{\partial t_{1}}{\partial \theta_{i}}=\cdots=\left(\frac{\partial g}{\partial t_{n}} \frac{\partial t_{n}}{\partial t_{n-1}} \cdots \frac{\partial t_{2}}{\partial t_{1}}\right) \frac{\partial t_{1}}{\partial \theta_{i}},
$$

to calculate the 'sensitivities' $\partial g / \partial t_{n}, \ldots, \partial g / \partial t_{1}$. The advantage in this case is that a full evaluation with respect to all parameters costs less than four times that of evaluating the objective function and this does not depend on the number of parameters $p$ which in the case of integrating out $\mathbf{S}$ is extremely large (Skaug and Fournier, 2006). The downside to this method is the increased memory usage required to store the $t_{1} \ldots, t_{n}$ which must be written to file in an initial evaluation of the objective function.

To observe a comparison let us calculate the partial derivatives of an example function

$$
y=g\left(x_{1}, x_{2}, x_{3}\right)=\exp \left(x_{1}^{2} x_{2}\right)+\left(x_{2} \cos x_{3}\right)^{2},
$$

which we break down into raw components

$$
\begin{aligned}
y & =\exp \left(x_{1}^{2} x_{2}\right)+\left(x_{2} \cos x_{3}\right)^{2}, \\
& =\exp \left(t_{1}^{2} t_{2}\right)+\left(t_{2} \cos t_{3}\right)^{2}, \\
& =\exp \left(t_{4} t_{2}\right)+\left(t_{2} t_{5}\right)^{2}, \\
& =\exp \left(t_{6}\right)+t_{7}^{2}, \\
& =t_{8}+t_{9}, \\
& =t_{10} .
\end{aligned}
$$

These values $\left(t_{1} \ldots, t_{10}\right)$ and the instructions as to how they were produced are saved as a data structure we call a directed graph which we can move through in a particular order. We draw this graph in Figure 4.1. The forward and reverse algorithms will go through from bottom to top and top to bottom respectively.

First we consider the forward algorithm to calculate the partial derivative $\frac{\partial y}{\partial x_{1}}=\frac{\partial t_{10}}{\partial x_{1}}$ in Algorithm 4.1. Note that to calculate all of the partial derivatives we need to rerun the algorithm two more times with different initial values (first 3 rows). This is not the case if we use the reverse algorithm as shown in Algorithm 4.2, where a single run calculates all required derivatives.

TMB uses the $\mathrm{C}++$ package $C p p A D($ Bell, 2005$)$ for derivative calculation due to its effectiveness in calculating higher order derivatives and the 


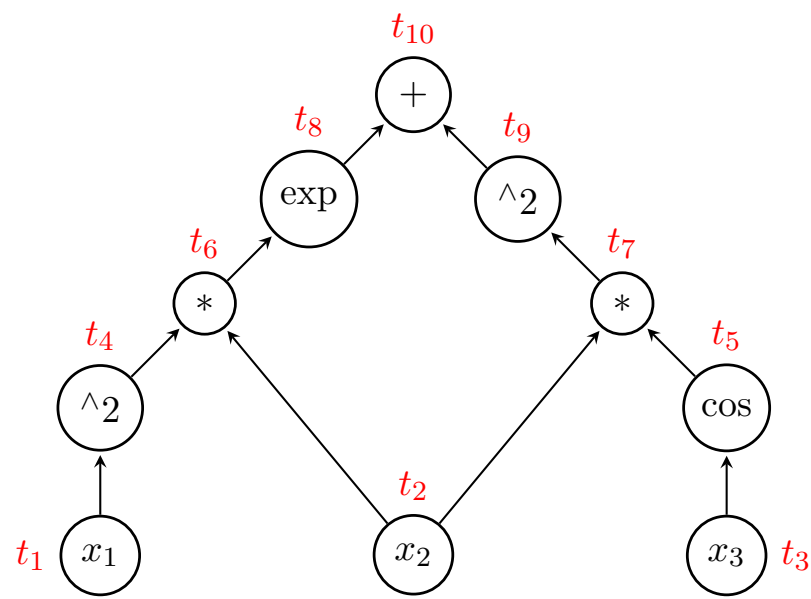

Figure 4.1: Directed graph of the function in (4.22).

Algorithm 4.1 Example of forward run to calculate partial derivative of (4.22).

$$
\begin{aligned}
\frac{\partial t_{1}}{\partial x_{1}} & =1 \\
\frac{\partial t_{2}}{\partial x_{1}} & =0 \\
\frac{\partial t_{3}}{\partial x_{1}} & =0 \\
\frac{\partial t_{4}}{\partial x_{1}} & =\frac{\partial t_{4}}{\partial t_{1}} \frac{\partial t_{1}}{\partial x_{1}}=2 t_{1} \\
\frac{\partial t_{5}}{\partial x_{1}} & =\frac{\partial t_{5}}{\partial t_{3}} \frac{\partial t_{3}}{\partial x_{1}}=0 \\
\frac{\partial t_{6}}{\partial x_{1}} & =\frac{\partial t_{6}}{\partial t_{4}} \frac{\partial t_{4}}{\partial x_{1}}+\frac{\partial t_{6}}{\partial t_{2}} \frac{\partial t_{2}}{\partial x_{1}}=2 t_{1} t_{2} \\
\frac{\partial t_{7}}{\partial x_{1}} & =\frac{\partial t_{7}}{\partial t_{2}} \frac{\partial t_{2}}{\partial x_{1}}+\frac{\partial t_{7}}{\partial t_{5}} \frac{\partial t_{5}}{\partial x_{1}}=0 \\
\frac{\partial t_{8}}{\partial x_{1}} & =\frac{\partial t_{8}}{\partial t_{6}} \frac{\partial t_{6}}{\partial x_{1}}=2 t_{1} t_{2} \exp \left(t_{6}\right), \\
\frac{\partial t_{9}}{\partial x_{1}} & =\frac{\partial t_{9}}{\partial t_{7}} \frac{\partial t_{7}}{\partial x_{1}}=0, \\
\frac{\partial t_{10}}{\partial x_{1}} & =\frac{\partial t_{10}}{\partial t_{8}} \frac{\partial t_{8}}{\partial x_{1}}+\frac{\partial t_{10}}{\partial t_{9}} \frac{\partial t_{9}}{\partial x_{1}}=2 t_{1} t_{2} \exp \left(t_{6}\right)=2 t_{1} t_{2} \exp \left(t_{1}^{2} t_{2}\right)
\end{aligned}
$$




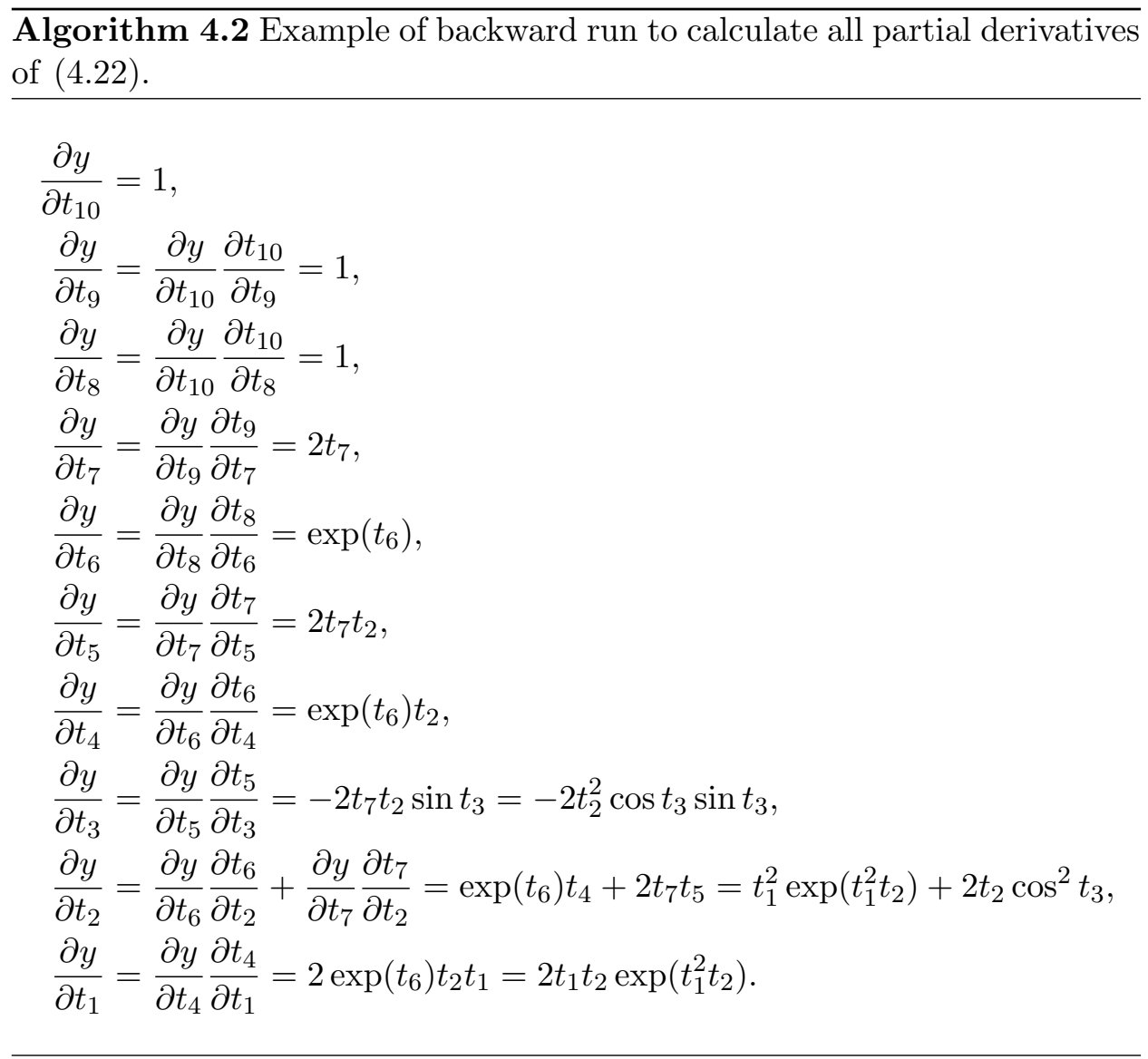


Eigen (Guennebaud and Jacob, 2010) library for C++ vector and matrix operations (Kristensen et al., 2016). The drawback to this structure is that implementation of parameter estimation within the TMB package requires writing the negative log-likelihood function in a . cpp file. Once written, the likelihood function can be compiled in $\mathrm{R}$ via built-in TMB functions and maximisation of the likelihood conducted using standard $\mathrm{R}$ functions such as optim.

The aim in TMB is to calculate both $l^{1}(\boldsymbol{\theta})$ and $\frac{\partial}{\partial \boldsymbol{\theta}} l^{1}(\boldsymbol{\theta})$ which requires calculation of the directed graphs for

$$
f(\mathbf{S}, \boldsymbol{\theta}), \quad \frac{\partial}{\partial \mathbf{S}} f(\mathbf{S}, \boldsymbol{\theta}), \quad \frac{\partial^{2}}{\partial \mathbf{S}^{2}} f(\mathbf{S}, \boldsymbol{\theta}) .
$$

These graphs are stored in memory and calculated only once. Evaluation of $l^{1}(\boldsymbol{\theta})$ requires a forward and reverse $\mathrm{AD}$ routine to calculate the first and second order derivatives of $f(\mathbf{S}, \boldsymbol{\theta})$ respectively. To calculate $\frac{\partial}{\partial \boldsymbol{\theta}} l^{1}(\boldsymbol{\theta})$, Kristensen et al. (2016) utilise the inverse subset algorithm which requires a reverse order sweep through the directed graph of $\frac{\partial^{2}}{\partial \mathbf{S}^{2}} f(\mathbf{S}, \boldsymbol{\theta})$. Further information on the method of $\mathrm{AD}$ for Laplace approximations to the likelihood function are available in Skaug and Fournier (2006), whilst TMB specific details outlined in Kristensen et al. (2016). More comprehensive discussions are provided by Griewank and Walther (2008).

\subsubsection{Implementation in Template Model Builder}

To implement our preferential model in TMB we write a so-called "User Template" which defines the negative joint log-likelihood. As mentioned in the previous section, this file must be written in a . cpp file using the $\mathrm{C}++$ library provided by TMB. We must first determine the distributions in our factorisation of the preferential joint likelihood, which is defined in (4.21) as $-\log ([\mathbf{X}, \mathbf{Y}, \mathbf{S}])=-\log ([\mathbf{Y} \mid \mathbf{S}, \mathbf{X}][\mathbf{X} \mid \mathbf{S}][\mathbf{S}])$. Under an assumed Gaussian model with sampling structure defined in (2.1) and Matérn covariance we have that

$$
\begin{aligned}
\mathbf{Y} \mid \mathbf{S}, \mathbf{X} & \sim N_{n}\left(\mathbf{S}_{0}, \tau^{2} I_{n}\right), \\
{[\mathbf{X} \mid \mathbf{S}] } & =f(\mathbf{S}), \\
\mathbf{S} & \sim N_{N}\left(\mathbf{D}_{N} \boldsymbol{\zeta}, \Sigma\right),
\end{aligned}
$$

where $\mathbf{S}$ is an $N$-dimensional discretisation of the continuous random field $S$, $\mathbf{S}_{0}$ are the values $\mathbf{S}$ at the $n$ sampling locations $\mathbf{X}$ and $f(\cdot)$ is some probability 
density function depending on $\mathbf{S}$ that defines the sampling structure. In the case of a point process model with intensity from (3.4) we would have

$$
[\mathbf{X} \mid \mathbf{S}]=\prod_{i=1}^{n} \frac{\exp \left\{\beta \mathbf{S}\left(\mathbf{X}_{i}\right)\right\}}{\int_{\mathcal{D}} \exp \{\beta \mathbf{S}(\mathbf{u})\} \mathrm{d} \mathbf{u}}
$$

The joint log-likelihood based on these densities can then be constructed as a function of the full set of field and preferential model parameters denoted $\boldsymbol{\theta}$. We also specify the latent variable to be integrated out of the model, which in our case is the discretised $\mathbf{S}$. We provide an example of a preferential . cpp file in Appendix C, with a multitude of nonpreferential model examples available at https://github.com/kaskr/ adcomp/tree/master/tmb_examples.

Once the joint log-likelihood has been specified we can conduct the remaining analysis in $R$ (R Core Team, 2013) by first compiling the . cpp file, which we call example.cpp here, and then loading it into the work space.

compile ("example.cpp")

dyn.load (dynlib ("example"))

Next we load in the data and give initial values to each of the parameters in the model. For sake of a visual example here we assume there are only two parameters called param1 and param2 whilst our latent field random effect $(\mathbf{S})$ is initialised as an $N$-dimensional vector of 0's.

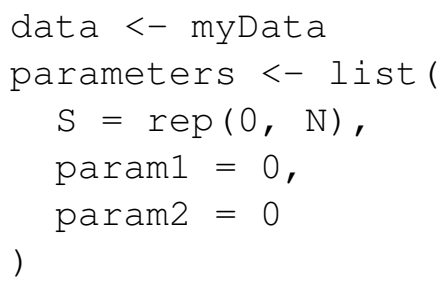

Finally we call MakeADFun which constructs both the Laplace approximation to the negative $\log$-likelihood function $-l^{1}(\boldsymbol{\theta})=-\log \left(L^{1}(\boldsymbol{\theta})\right)$ from (4.20) and the respective gradient function, for the chosen random effect. This new object denoted obj below can then be minimised using an $R$ optimisation method.

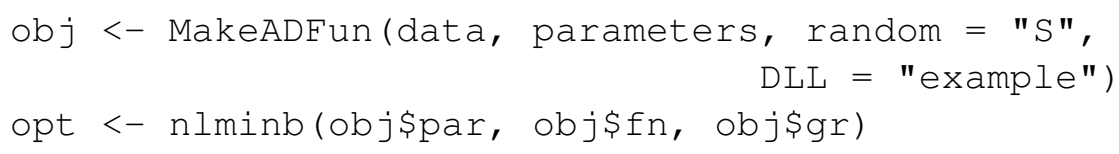




\subsection{Simulation Comparison}

To illustrate the performance of TMB purely in terms of parameter estimation and facilitate comparison with other results in the literature, we performed a simulation study following similar settings from Section 3 of Diggle et al. (2010).

\subsubsection{Data Description}

Our data were generated following model (2.1) with nugget variance $\tau^{2}=$ 0.10 . The process $S$ had a Matérn correlation structure with scale (range) $\phi=0.15$, known shape parameter $\kappa=1$ and variance $\sigma^{2}=1.5$. The field was simulated over a $91 \times 91$ discrete grid of equally-spaced points on the unit square. Conditional on each realization of $S$ we obtained $n=100$

sampling locations $\mathbf{X}$ generated using the location intensity function from (3.4).

We conducted two separate simulations using the same realisations of $S$. A preferential simulation in which the sampling locations were determined with a value of $\beta=1.5$ in the location intensity function (3.4), and a nonpreferential sampling simulation where $\beta=0$. Note that in the the nonpreferential case we have uniform random sampling. Examples of a single realisation of $S$ in each case can be seen in Figure 4.2 .

In total we generated 500 samples of $S$ and applied the standard maximum likelihood method (ignoring any possible preferential nature in the sampling), and also TMB, which allows for the inclusion of a potential preferential effect, to both preferentially and non-preferentially sampled data sets. We applied the TMB model using a grid size of $31 \times 31$ to approximate the integral in the denominator of $(3.4)$, where this size was used to balance computational efficiency and model performance. For this particular simulation analysis, increasing the grid density for the model showed little to no change in the parameter estimates.

\subsubsection{Parameter Estimation}

The parameter estimate comparisons are shown in Figures 4.3 .4 .4 for the preferential and non-preferentially simulated data sets, respectively. To compare the performance of TMB to other alternatives we consider parameter estimates using the same preferential model but implemented via Integrated Nested Laplace Approximations (INLA) (Rue et al., 2009) in the R-INLA package (www.r-inla.org), and also those obtained using the original MC 


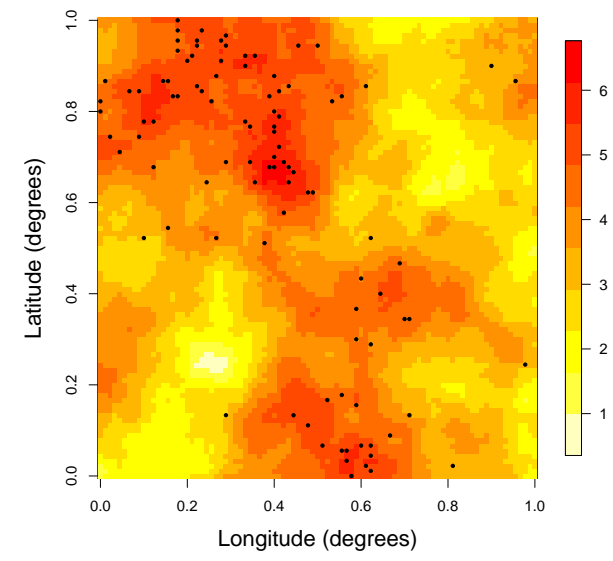

(a) Preferentially sampled $(\beta=1.5)$

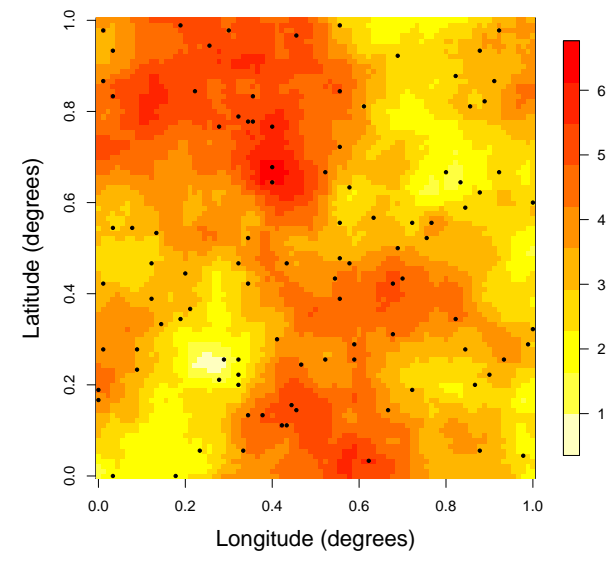

(b) Non-preferentially sampled $(\beta=0)$

Figure 4.2: Example of a single realisation of $S$ and corresponding 100 sampling locations generated using the preferential $(\beta=1.5)$ and nonpreferential $(\beta=0)$ approach described in Section 4.5.1.

method from Diggle et al. (2010). When using R-INLA we adapted the code available on-line at http: / / www.r-inla.org/examples/case-studies / diggle09/simulation1. The true value of each parameter is indicated with a black horizontal line and initial parameter values for optimisation were taken to be the standard MLE field parameters and true preferential parameter $\beta$.

As expected, we see that when the location measurements were preferentially sampled the standard MLE approach showed a significant systematic bias for estimating all parameters in the model (with the notable exception of the nugget variance $\tau^{2}$ ). We also note that the performance of both R-INLA and TMB showed considerable success, particularly in estimating the mean parameter $\mu$. The results obtained with the MC likelihood approximation of Diggle et al. (2010) confirm our concerns in Section 4.3 and although they showed some correction, this was not to the level of TMB or $\mathrm{R}$-INLA. The results for the case of non-preferentially sampled locations are also reassuring-all estimation methods are essentially equivalent and not over correcting for any possible preferentiability.

We also assess coverage of $95 \%$ confidence intervals for $\mu$ and $\beta$ across the 500 preferentially and non-preferentially simulated data sets, where we 


\subsection{Simulation Comparison}

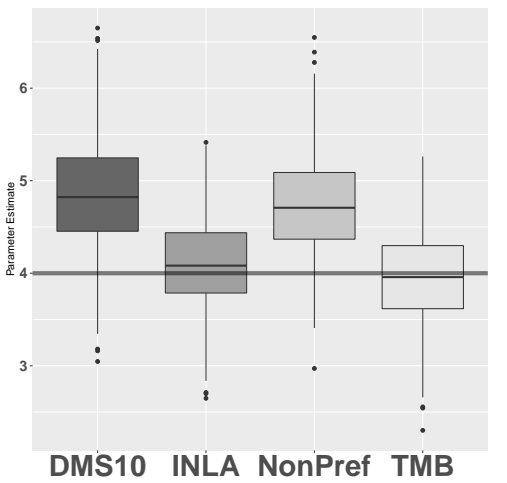

(a) Mean $(\mu)$

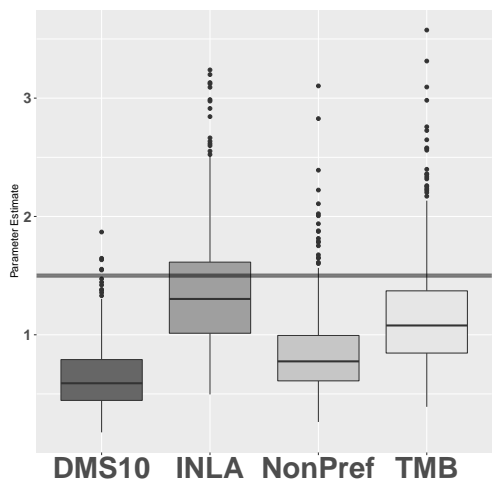

(c) Variance $\left(\sigma^{2}\right)$

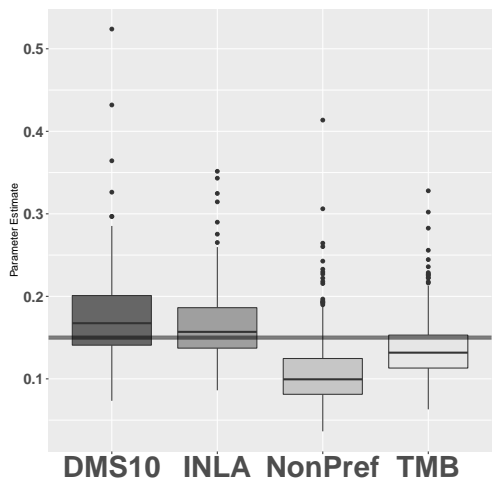

(b) Scale $(\phi)$

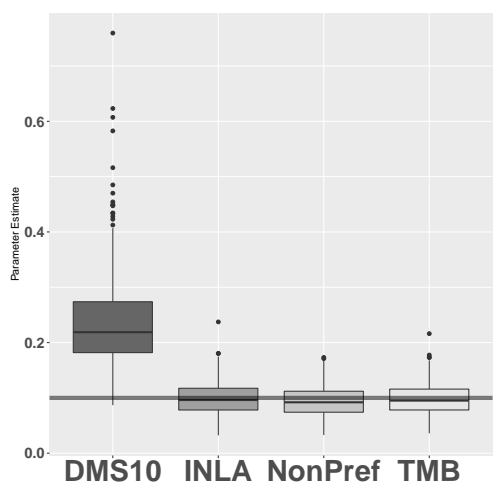

(d) Nugget variance $\left(\tau^{2}\right)$

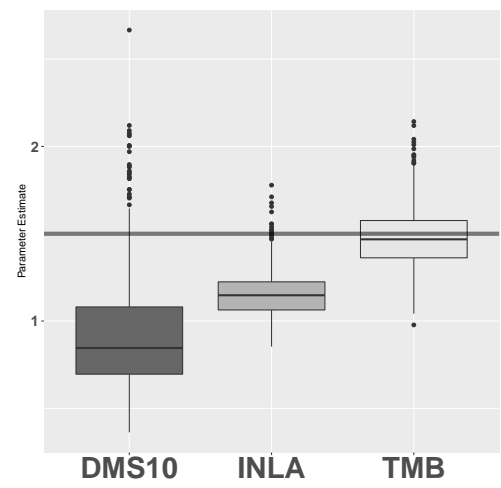

(e) Preferential sampling $(\beta)$

Figure 4.3: Parameter estimates over 500 preferentially simulated data sets with true parameter values marked as black lines. The abbreviations DMS 10 and NonPref stand for the Monte Carlo method of Diggle et al. (2010) and standard MLE (non-preferential) estimation respectively. 


\subsection{Simulation Comparison}

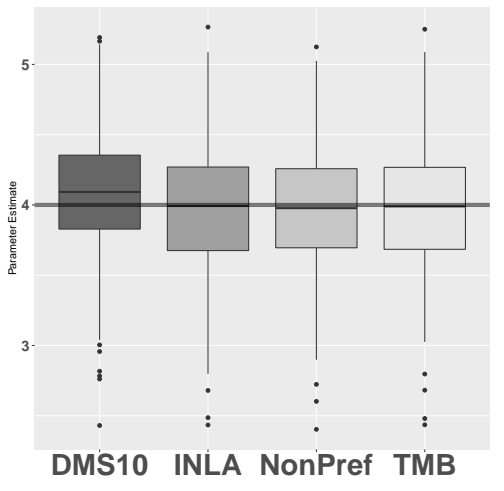

(a) Mean $(\mu)$

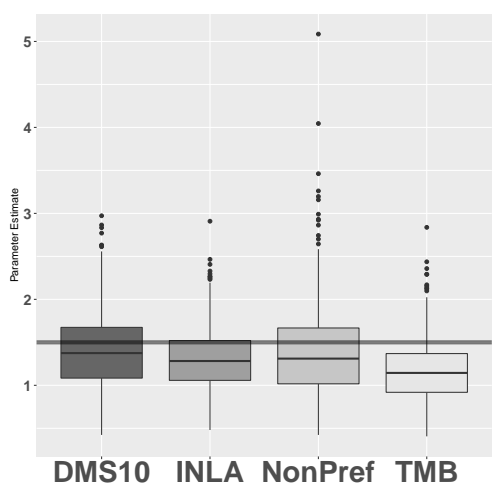

(c) Variance $\left(\sigma^{2}\right)$

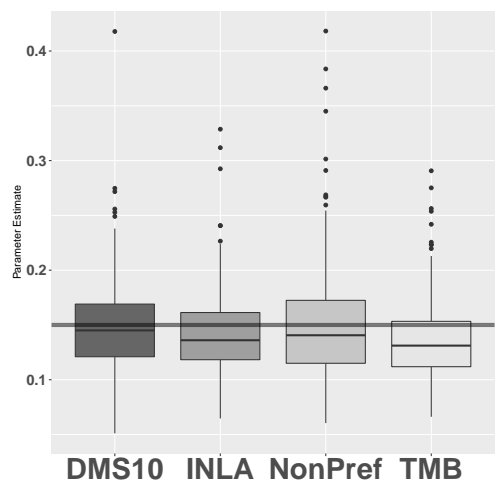

(b) Scale $(\phi)$

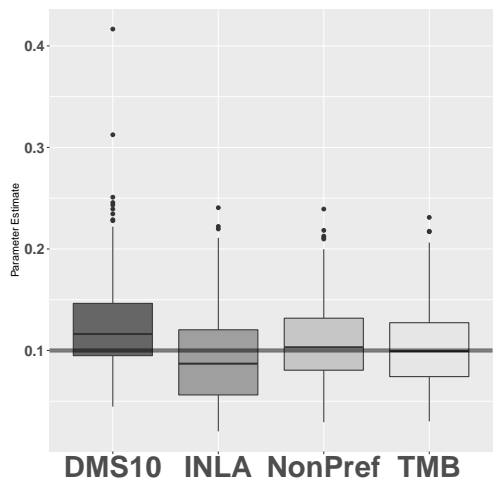

(d) Nugget variance $\left(\tau^{2}\right)$

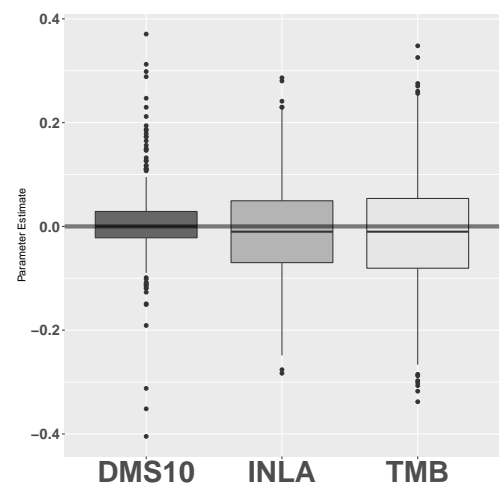

(e) Preferential sampling $(\beta)$

Figure 4.4: Parameter estimates over 500 non-preferentially simulated data sets with true parameter values marked as black lines. The abbreviations DMS10 and NonPref stand for the Monte Carlo method of Diggle et al. (2010) and standard MLE (non-preferential) estimation respectively. 
compute approximate intervals on each data set as the parameter estimate plus/minus two standard errors. We compare intervals from TMB with the standard MLE's. In the non-preferential data set the coverage of $\mu=4$ was $88 \%$ for both models, whilst the coverage of $\beta=0$ using TMB was $95 \%$. In the preferential simulation the coverage of $\mu=4$ was $88 \%$ and $42 \%$ for the preferential model in TMB and standard MLE's respectively, which makes sense when observing Figure 4.3. The coverage of $\beta=1.5$ was $92 \%$. Note the identical coverage of $\mu$ using TMB across the two data sets, which further highlights the consistently accurate estimation of $\mu$ across preferentially and non-preferentially sampled data. An alternative approach to construct confidence intervals could be via using likelihood ratios on the profile likelihood function.

Computationally, although R-INLA runs faster and is easily upscalable to finer grids, the flexibility of defining the sampling model structure directly in TMB is not found elsewhere and it enables the use of more complex preferential sampling models. The computational time to estimate parameters for a single simulation in this section took approximately 17 seconds in R-INLA and 38 seconds in TMB on our desktop Windows 10 computer running an Intel i7-4790 CPU at 3.60GHz with 16 GB of RAM. The Monte Carlo method of Diggle et al. (2010) failed to converge to an appropriate level within our iterations using $m=10,000$ Monte Carlo simulations per iteration. However capping the iterations to a maximum of 200 took approximately 1945 seconds.

\subsection{Application to the Galicia Moss Data}

In this section we illustrate our approach on a data set analysed previously in Diggle et al. (2010). The observations consist of lead concentrations (micrograms per gram dry weight) in samples of moss in Galicia, Northern Spain. These measurements were taken in two separate surveys conducted in October 1997 and July 2000 and Figure 4.5 shows the sampling locations of each year. Since the 1997 sample data was collected more intensively in regions of expected high lead concentrations, it was flagged as potentially being preferentially sampled. The second survey used an approximately regular lattice design so was not considered preferential.

We used TMB to analyse the combined data using the same model assumptions as in Diggle et al. (2010). More specifically, we use (2.1), where the underlying signal $S$ is a zero-mean stationary Gaussian process with variance $\sigma^{2}$ and Matérn correlation function with scale (range) $\phi$ and known 


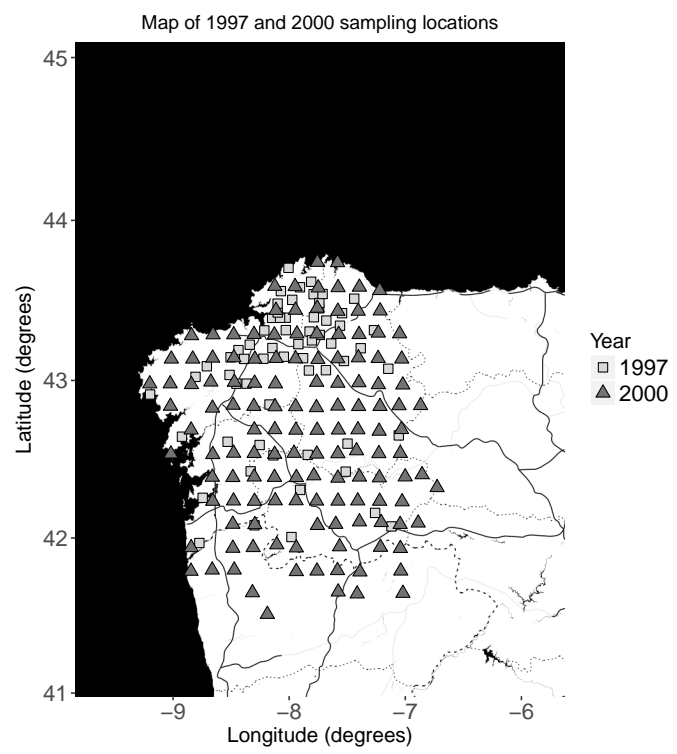

Figure 4.5: Survey sampling locations for 1997 and 2000 lead concentration data sets. Map tiles by Stamen Design, under CC BY 3.0. Data by OpenStreetMap, under ODbL.

shape parameter $\kappa=0.5$. The measurement errors were assumed to be Gaussian with mean 0 and nugget variance $\tau^{2}$. Following the original analysis, we treated the 1997 and 2000 data sets as preferentially and nonpreferentially sampled respectively and assumed that the two data sets share the parameters $\sigma, \phi$ and $\tau$, but that they may have different means $\mu_{97}, \mu_{00}$. Conditional on $S$ we assumed that the 1997 sampling locations had a location intensity defined in (3.4) for some $\beta \in \mathbb{R}$.

The results in Table 4.1 show the parameter estimates, standard errors

Table 4.1: Parameter estimates, standard errors and correlations for the joint 1997 and 2000 data model using TMB.

\begin{tabular}{|c|c|c|cccccc|}
\hline Parameter & Estimate & Standard Error & \multicolumn{5}{|c|}{ Correlation Matrix } \\
\hline$\mu_{97}$ & 1.761 & 0.159 & 1.000 & -0.016 & 0.252 & -0.177 & -0.193 & 0.245 \\
$\mu_{00}$ & 0.728 & 0.098 & & 1.000 & 0.051 & 0.180 & 0.045 & -0.056 \\
$\log \sigma$ & -1.281 & 0.178 & & & 1.000 & -0.091 & -0.513 & 0.501 \\
$\log \phi$ & -1.113 & 0.367 & & & & 1.000 & 0.504 & -0.488 \\
$\log \tau$ & -1.066 & 0.367 & & & & & 1.000 & -0.502 \\
$\beta$ & -3.818 & 0.962 & & & & & & 1.000 \\
\hline
\end{tabular}


and correlation matrix using TMB. Here, the standard errors and correlations are computed using the inverse of the observed information matrix calculated by TMB. These results are similar to those obtained by the original MC method in Diggle et al. (2010). Taking a measure of the degree of preferentiability as $|\beta \sigma|$, we obtain values of 0.815 and 1.060 using ours and Diggle et al. (2010)'s results respectively. There is a significant, albeit small difference between these two values.

We believe that the TMB estimates in Table 4.1 are more reliable and provide a more accurate representation of the uncertainty in our estimates. This is due to the ease at which TMB can maximise over the smooth surface of the Laplace Approximation, compared with the difficulty of optimising the likelihood with $\mathrm{MC}$ variability. We are also able to efficiently maximise the likelihood using TMB from initial parameters obtained via standard MLE methods for the field parameters. 


\section{Chapter 5}

\section{Spatial Prediction Under Preferential Sampling}

Parameter estimation is only one element of spatial data analysis, with prediction of the process $S$ at unobserved locations often being the primary goal of inference. We must take care when predicting processes which have been sampled in a preferential manner, since in this case the predictive distribution $[S \mid \mathbf{X}, \mathbf{Y}]$ must take into account the dependency between the process and sampling locations. In Section 5.1 we cover in detail the derivation of $[S \mid \mathbf{X}, \mathbf{Y}]$ under standard (non-preferential) assumptions. However, in Section 5.2 we show that one cannot assume the predictive distribution $[S \mid \mathbf{X}, \mathbf{Y}]$ derived in Section 5.1 will provide an accurate representation of a preferetially sampled field, even when we have corrected for bias in the parameter estimates. In fact, we show how $[S \mid \mathbf{X}, \mathbf{Y}]$ under preferential sampling is intractable and propose prediction from this distribution using the mode estimated as part of the Laplace approximation detailed in Section 4.4. Lastly, we assess prediction from both our preferential and non-preferential prediction models across various simulated data sets in Section 5.3 and analyse the differences between the predictive distributions in Section 5.4 .

\subsection{Naive Prediction}

Section 4.3 .2 touched upon the predictive distribution of $[S \mid \mathbf{X}, \mathbf{Y}]$ in Diggle et al. (2010) which took the multivariate normal form defined in (4.8). Taking $\mathbb{E}[S \mid \mathbf{X}, \mathbf{Y}]$ and $\operatorname{Var}(S \mid \mathbf{X}, \mathbf{Y})$ from $(4.8)$ as the point prediction and prediction variance of the field $S$ given the data $\mathbf{X}$ and $\mathbf{Y}$, is the same as the using the minimum mean square kriging estimator defined in (2.11) and (2.12) for the constant mean trend case under no preferential effect. That is, the prediction at a location $\mathbf{u} \in \mathcal{D} \subseteq \mathbb{R}^{2}$ can be written as a linear combination

$$
\hat{S}(\mathbf{u})=\mu+\sum_{i=1}^{n} w_{i}(\mathbf{u})\left(Y_{i}-\mu\right),
$$


where $w_{i}$ is the $i$ th element of an $n$-dimensional vector $\mathbf{w}(\mathbf{u})=\sigma^{2} \mathbf{r}^{\top}\left(\Sigma_{n}+\right.$ $\left.\tau^{2} I_{n}\right)^{-1}$ for the $n \times n$ covariance matrix $\Sigma_{n}$ and $r_{i}=\rho\left(\left\|\mathbf{u}-\mathbf{x}_{i}\right\|\right)$. If we have a spatial trend surface $\mu(\mathbf{X})=\mathbf{D} \boldsymbol{\zeta}$ we may also include a varying trend equivalent which was defined in (2.14) such that prediction is

$$
\hat{S}(\mathbf{u})=\mathbf{D}_{u} \boldsymbol{\zeta}+\sum_{i=1}^{n} w_{i}(\mathbf{u})\left(Y_{i}-\mathbf{D}_{i} \boldsymbol{\zeta}\right),
$$

where $\mathbf{D}_{u}^{\top}$ and $\mathbf{D}_{i}^{\top}$ are $p$-dimensional vectors of covariates at location $\mathbf{u}$ and the $i$ th sampling location respectively, $\zeta$ a $p$-dimensional vector of coefficients.

These distributions are derived by first noting that since $\mathbf{S}$ and $\mathbf{X}$ are independent we can write $\mathbf{S} \mid \mathbf{X}=\mathbf{S} \sim N_{N}\left(\mathbf{D}_{N} \boldsymbol{\zeta}, \Sigma_{N}\right)$, where $\Sigma_{N}$ is the $N \times N$ covariance matrix for the discretised grid of $\mathbf{S}$. Secondly $\mathbf{Y} \mid \mathbf{S}, \mathbf{X} \sim$ $N_{n}\left(S_{0}, \tau^{2} I_{n}\right)$ therefore

$$
[\mathbf{Y} \mid \mathbf{X}]=\mathbb{E}_{\mathbf{S} \mid \mathbf{X}}[[\mathbf{Y} \mid \mathbf{S}, \mathbf{X}]]=\mathbb{E}_{\mathbf{S}}[[\mathbf{Y} \mid \mathbf{S}, \mathbf{X}]] \sim N_{n}\left(\mathbf{D}_{n} \boldsymbol{\zeta}, \Sigma_{n}+\tau^{2} I_{n}\right) .
$$

Note that $[\mathbf{Y} \mid \mathbf{S}, \mathbf{X}]$ does not depend on whether or not preferential sampling exists under our current model but $[\mathbf{Y} \mid \mathbf{X}]$ does.

Next, let us define $\boldsymbol{C}$ as an $n \times N$ matrix whose $i$ th row contains $(N-1)$ 0 's and a single 1 indicating the position of the $i$ th observed location within the lattice. Then by denoting $\mathbf{C} \Sigma_{N}=\Sigma_{n N}$ and $\Sigma_{N} \mathbf{C}^{T}=\Sigma_{N n}$ to be $n \times N$ and $N \times n$ matrices respectively, then we can write the joint distribution

$$
\left(\begin{array}{c}
\mathbf{Y} \mid \mathbf{X} \\
\mathbf{S} \mid \mathbf{X}
\end{array}\right)=\left(\begin{array}{c}
\mathbf{Y} \mid \mathbf{X} \\
\mathbf{S}
\end{array}\right) \sim N_{N+n}\left(\left(\begin{array}{c}
\mathbf{D}_{n} \boldsymbol{\zeta} \\
\mathbf{D}_{N} \boldsymbol{\zeta}
\end{array}\right),\left(\begin{array}{cc}
\Sigma_{n}+\tau^{2} I_{n} & \Sigma_{n N} \\
\Sigma_{N n} & \Sigma_{N}
\end{array}\right)\right)
$$

Finally by using the properties of the conditional multivariate normal density we can derive the distribution of $[\mathbf{S} \mid \mathbf{X}, \mathbf{Y}]$ shown in (4.8) and obtain the kriging prediction in $(5.2)$ by taking the expected value.

To use these predictive distributions one may simply plug-in the latent field parameter estimates to obtain the prediction $\hat{S}(\mathbf{u})$. The mean parameter $(\hat{\mu}$ or $\hat{\boldsymbol{\zeta}})$ and nugget estimates $\hat{\tau}$ can be seen in the linear predictive definitions of (5.1)-(5.2), whilst if we assume a Matérn model the covariance parameter estimates $\phi, \hat{\sigma}$ and $\hat{\kappa}$ are used to obtain the form of $\Sigma_{N}$.

Under preferential sampling one might be inclined to simply correct the parameter estimates using a model such as the one described in Section 4.4 , then plug these "corrected" parameter estimates into (5.1) or (5.2) depending on your mean trend specification. Since kriging assumes that the sampling locations do not provide information in the predictive distribution of 
the field it can simply be viewed as an extrapolation of the data to the prediction location based on the knowledge of the properties of the underlying field we want to predict. Doing this however still ignores the relationship between the process and the sampling locations, which provides valuable predictive information. Predictive models that utilise the relationship between the latent field and sampling locations have more information to make the predictions with. For example, a model that identifies sampling intensity in regions of higher field values now has information that the sparsely sampled regions likely have lower field values. This may reduce the predicted values in this region compared to simply extrapolating nearby measurements, even with correct parameter values. We show in Section 5.2 that the predictive distribution under preferential sampling is more complex than the kriging approach shown in this section and propose a method to obtain predictions in this case.

\subsection{Preferential Predictive Distribution}

Under preferential sampling we cannot follow the same method to derive $[\mathbf{S} \mid \mathbf{Y}, \mathbf{X}]$ as shown in (5.4). As mentioned in Section 4.3 .2 one issue is that $[\mathbf{Y} \mid \mathbf{X}]$ does not follow $(5.3)$ since $[\mathbf{S} \mid \mathbf{X}] \neq[\mathbf{S}]$, which is key to the calculation of the expectation in this case. Under preferential sampling we need to calculate $[\mathbf{Y} \mid \mathbf{X}]=\mathbb{E}_{\mathbf{S} \mid \mathbf{X}}[[\mathbf{Y} \mid \mathbf{S}, \mathbf{X}]]$ which is difficult, since $[\mathbf{S} \mid \mathbf{X}]$ is itself intractable. Consequently, we cannot assume that $[\mathbf{S} \mid \mathbf{X}, \mathbf{Y}]$ is the same as the non-preferential case in (4.8) and this distribution appears to be intractable.

Alternatively we could take the mode of the intractable predictive distribution $[\mathbf{S} \mid \mathbf{X}, \mathbf{Y}]$ as our point predictions. This option ties in with the Laplace approximation method used to evaluate the preferential likelihood function described in Section 4.4. Note that in the Laplace approximation to the likelihood function for a given parameter $\boldsymbol{\theta}$, we calculate $\hat{\mathbf{S}}(\boldsymbol{\theta})$ defined in (4.14). If we explicitly write out this function in the case of the preferential 
likelihood evaluation we get

$$
\begin{aligned}
\hat{\mathbf{S}}(\boldsymbol{\theta}) & =\underset{\mathbf{S}}{\arg \min } f(\mathbf{S}, \boldsymbol{\theta}), \\
& =\underset{\mathbf{S}}{\arg \min }-\log ([\mathbf{S}, \mathbf{X}, \mathbf{Y}]), \\
& =\underset{\mathbf{S}}{\arg \min }-\log ([\mathbf{S} \mid \mathbf{X}, \mathbf{Y}][\mathbf{X}, \mathbf{Y}]), \\
& =\underset{\mathbf{S}}{\arg \min }-\log ([\mathbf{S} \mid \mathbf{X}, \mathbf{Y}])-\log ([\mathbf{X}, \mathbf{Y}]), \\
& =\underset{\mathbf{S}}{\arg \min }-\log ([\mathbf{S} \mid \mathbf{X}, \mathbf{Y}]), \\
& =\underset{\mathbf{S}}{\arg \max } \log ([\mathbf{S} \mid \mathbf{X}, \mathbf{Y}]), \\
& =\underset{\mathbf{S}}{\arg \max }[\mathbf{S} \mid \mathbf{X}, \mathbf{Y}] .
\end{aligned}
$$

Here we can see that $\hat{\mathbf{S}}(\boldsymbol{\theta})$ is in fact the mode of $[\mathbf{S} \mid \mathbf{X}, \mathbf{Y}]$ for the parameter $\boldsymbol{\theta}$.

If we define $\boldsymbol{\theta}_{\text {opt }}$ to be the parameters that optimise the preferential likelihood function we note that $\hat{\mathbf{S}}\left(\boldsymbol{\theta}_{\text {opt }}\right)$ is the mode of $[\mathbf{S} \mid \mathbf{Y}, \mathbf{X}]$ for the parameters that maximise the fully specified likelihood, hence $\hat{\mathbf{S}}\left(\boldsymbol{\theta}_{\text {opt }}\right)$ yields a predictor for the random field $S$ that takes into account the preferential sampling effect. This is similar to kriging in that our predictions depend on the field parameter estimates that maximise a likelihood function, except in this case the predictive distribution also utilises the preferential parameter. In Section 5.3 we compare the predictive performance using this method with kriging and follow in Section 5.4 with a direct comparison between the two predictive distributions when using the same parameters.

\subsection{Simulation Comparison}

In this section we run the same simulation as detailed in Section 4.5.1, but this time compare predictions from preferential and non-preferential models, both with a constant mean trend.

\section{Simulation Prediction results}

For the non-preferential model we obtained predictions using ordinary kriging with a full set of plug-in parameters for the mean and covariance structure. These parameters were obtained using the standard maximum likelihood procedure and are the non-preferential parameter estimates from Sec- 


\subsection{Simulation Comparison}

Table 5.1: Comparison of approximate 95\% confidence intervals for the prediction errors (PE) and bias between standard MLE and TMB over 500 independent simulations for preferential $(\beta=1.5)$ and non-preferential $(\beta=0)$ models.

\begin{tabular}{|c|c|c|c|}
\hline Sampling System & Parameter & Standard MLE & TMB \\
\hline Preferential & Bias & $(0.64,0.79)$ & $(0.11,0.21)$ \\
Preferential & PE & $(0.75,0.88)$ & $(0.40,0.47)$ \\
\hline Non-Preferential & Bias & $(-0.03,0.05)$ & $(-0.03,0.06)$ \\
Non-Preferential & PE & $(0.32,0.37)$ & $(0.32,0.38)$ \\
\hline
\end{tabular}

tion 4.5.2. For our preferential model using TMB our predictions were taken as $\hat{\mathbf{S}}\left(\boldsymbol{\theta}_{\text {opt }}\right)$, where $\hat{\mathbf{S}}$ is defined in (4.14) and $\boldsymbol{\theta}_{\text {opt }}$ are the parameters that maximised the TMB likelihood function for that particular data set. These parameters are the preferential estimates in Section 4.5.2. As noted in Section $5.2, \hat{\mathbf{S}}\left(\boldsymbol{\theta}_{\text {opt }}\right)$ is the mode of $[\mathbf{S} \mid \mathbf{Y}, \mathbf{X}]$ for $\boldsymbol{\theta}=\boldsymbol{\theta}_{\text {opt }}$.

To compare point predictions from each of the two methods we compare the bias and the prediction error (PE) defined for the $j$ th field as

$$
\begin{aligned}
\operatorname{PE}_{j}\left(\mathbf{x}_{0}\right) & =\sqrt{\left(\hat{\mathbf{S}}_{j}\left(\mathbf{x}_{0}\right)-\mathbf{S}_{j}\left(\mathbf{x}_{0}\right)\right)^{2}}, \\
\operatorname{Bias}_{j}\left(\mathbf{x}_{0}\right) & =\hat{\mathbf{S}}_{j}\left(\mathbf{x}_{0}\right)-\mathbf{S}_{j}\left(\mathbf{x}_{0}\right),
\end{aligned}
$$

at location $x_{0}=(0.50,0.50)$. Approximate $95 \%$ confidence intervals for these measures are shown in Table 5.1 for both preferentially and nonpreferentially sampled processes. Following Diggle et al. (2010), these intervals were calculated as the mean value over the 500 simulations, plus or minus 2 standard errors.

The results in Table 5.1 reveal a considerable improvement in prediction accuracy, both in terms of bias and prediction errors when using TMB for the preferentially sampled example. In addition, when there was no preferential sampling present, TMB adjusted accordingly and performed almost identically to standard methods.

Although the point predictions in Table 5.1 show the benefit in terms of bias correction, we are not considering the uncertainty in these predictions. To assess the performance of the prediction methods above whilst accounting for their associated uncertainty, we use ignorance scores (Roulston and Smith, 2002). This measure, sometimes called the logarithmic score, is defined as:

$$
\operatorname{IGN}(x)=-\log (p(x))
$$


where $p$ is the predictive density and $x$ is the target forecast (Gneiting and Raftery, 2007; Siegert et al., 2014). An alternative approach could be to consider coverage of prediction intervals. However, we consider ignorance scores to reward models for smaller prediction variances in accurately estimated regions. We believe that finding the balance between informative and inflated prediction variances, which would result in high coverage but be of little value, to be clearer using the ignorance score approach.

For each simulation we calculate the mean ignorance score (MIGN) of our latent field predictions as

$$
\operatorname{MIGN}_{j}=\frac{1}{N} \sum_{i=1}^{N}\left\{\frac{\left(S_{j, i}-\hat{S}_{j, i}\right)^{2}}{\sigma_{j, i}^{2}}+2 \log \sigma_{j, i}\right\},
$$

where $S_{j, i}$ is the true value of field $S_{j}$ at the $i$ th lattice location, $\hat{S}_{j, i}$ is the corresponding predicted value and $\sigma_{j, i}^{2}$ is the prediction variance of $\hat{S}_{j, i}$. Figure 5.1 compares the MIGN scores for both models across the 500 preferential and non-preferential data sets, where lower MIGN's are better. We see that the preferential TMB model performed significantly better in the preferentially sampled data sets, supporting the observed improvement in point predictions from Table 5.1. In addition, performance on the nonpreferentially sampled data sets was almost identical between the two methods, showing little drawback in using the preferential model for cases where no preferential sampling was present.

\subsection{Comparison Between Predictive Distributions}

The objective of this section is to illustrate the impact of naively using the non-preferential distribution in (4.8) as an approximation to the correct $[\mathbf{S} \mid \mathbf{Y}, \mathbf{X}]$, when computing predictions at unobserved locations. As discussed in Section 5.2, such a choice impacts negatively the parameter estimates due to the incorrect specification of the likelihood. Below we will show that it also provides inadequate results when used to predict the field even when the parameters are estimated correctly.

Using the same simulation as in the previous section, we compare spatial prediction using the non-preferential conditional distribution from (4.8) but with a constant mean trend $\mu$, equivalent to ordinary kriging, with the mode of the estimated $[\mathbf{S} \mid \mathbf{Y}, \mathbf{X}]$ from TMB, which accounts for the preferential effect. The latter is the prediction method described in Section 5.2. In 


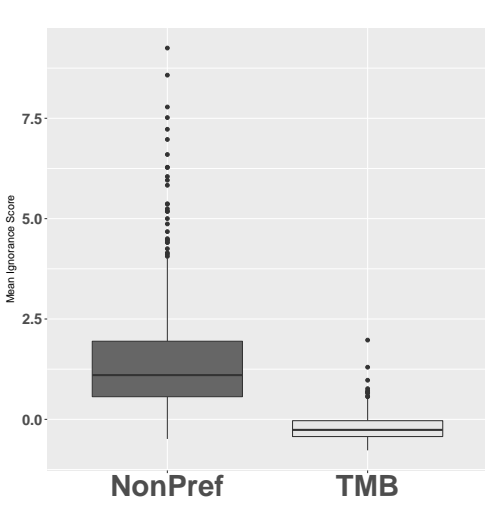

(a) Preferentially Sampled

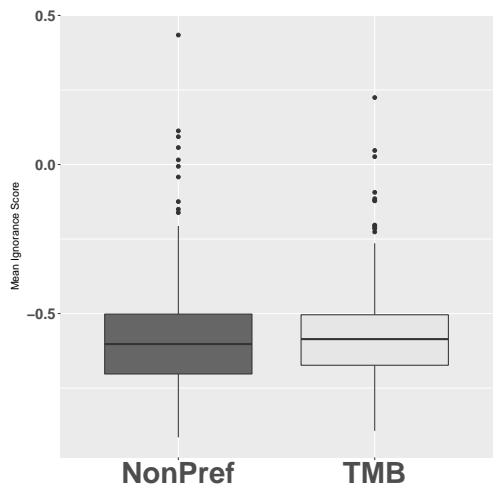

(b) Non-Preferentially Sampled

Figure 5.1: Comparison between mean ignorance scores across all 500 data sets in the preferentially sampled case (left panel) and non-preferentially sampled (right panel).

this case however, identical field parameter estimates were used for the two prediction methods, with the preferential parameter $\beta$ not utilised in the non-preferential model. These parameter estimates were obtained using the correct preferential model in TMB and are therefore corrected to account for the sampling bias.

For each sample we first compute the root-mean-squared prediction error

$$
\operatorname{RMSPE}_{j}=\frac{1}{N} \sum_{i=1}^{N} \sqrt{\left(S_{j, i}-\hat{S}_{j, i}\right)^{2}},
$$

where $S_{j, i}$ and $\hat{S}_{j, i}$ are as in $(5.5)$. The 500 values of $\mathrm{RMSPE}_{j}$ for each of the two prediction methods are show in Panel (a) of Figure 5.2. To also take into account the prediction uncertainty, Panel (b) of the same Figure includes the boxplot of the corresponding values of MIGN as defined in (5.5). We see that utilising a correct specification of the distribution of $\mathbf{S} \mid \mathbf{Y}, \mathbf{X}$ results in large gains in prediction accuracy.

To illustrate the nature of the difference between the two sets of predictions we show one instance of our simulation study in Figure 5.3. Panel (a) contains the values $\mathbf{S}$ of the true field on the grid, Panel (b) contains the predictions using the mode of the conditional distribution of $\mathbf{S} \mid \mathbf{Y}, \mathbf{X}$, while Panel (c) contains the results using the non-preferential kriging distribution from (4.8) but with the correct TMB parameters. Note, for example, the low 


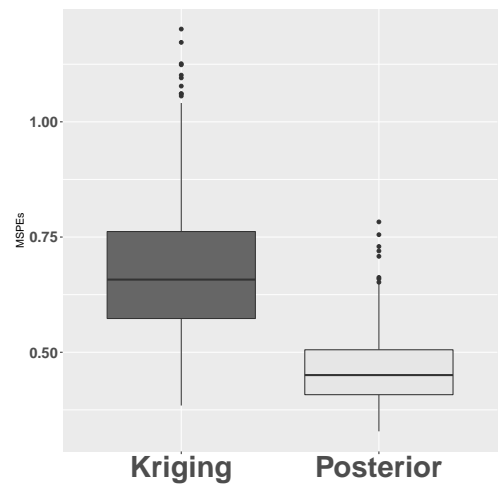

(a) RMSPEs

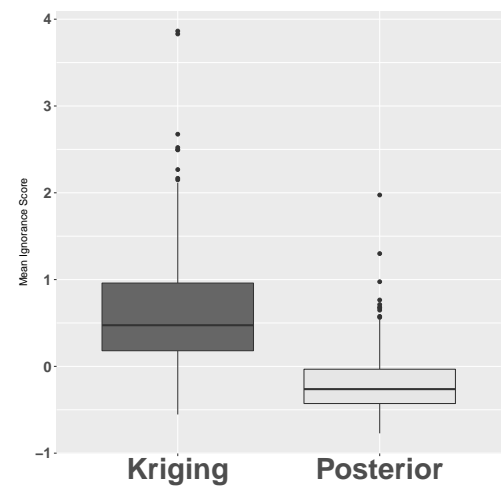

(b) MIGN

Figure 5.2: Comparison of root-mean-square prediction error and mean ignorance scores using corrected parameter estimates and different formulations for $[\mathbf{S} \mid \mathbf{Y}, \mathbf{X}]$.

temperature region in the South-West corner of the domain which has few sampled locations. The naive prediction simply extrapolated the temperature between the nearby sampled points resulting in overestimation of the temperature in this region, even with the corrected field parameters. In contrast, predictions using the preferential posterior from TMB adjusted better to this extremity using information from the location of sampled points and their dependency on the field. For this sample, the mean ignorance score (MIGN) was -0.44 when using the predictions from TMB and 0.31 for the non-preferential equivalent.

These differences can be explained intuitively by noting that in a preferential sampling setting the observed locations $\mathbf{X}$ carry information on the values of the field $S$. For example, in our simulation setting, we expect $S$ to take higher values near $\mathbf{X}$, and also lower ones away from $\mathbf{X}$. The predictions based on (4.8) do not reflect the latter. 


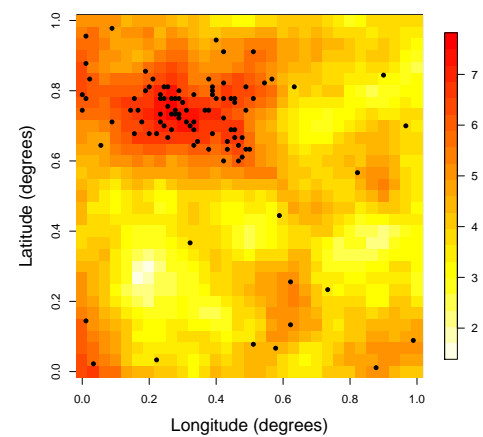

(a) True Field

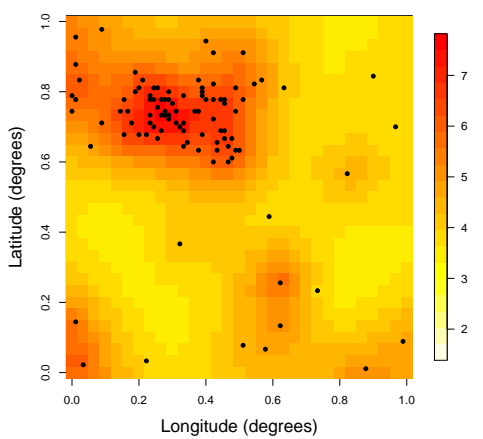

(b) Preferential Posterior Prediction

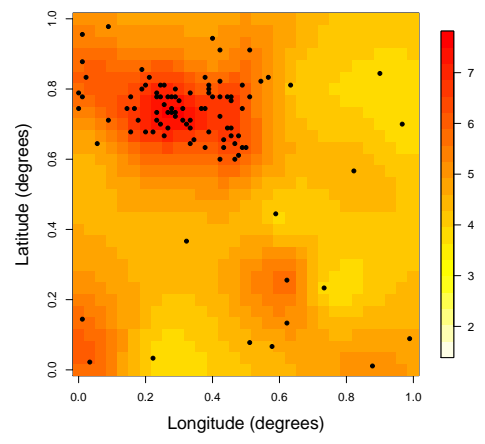

(c) Non-Preferential Prediction (Kriging)

Figure 5.3: Comparison between true field (a) and both preferential (b) and non-preferential (c) predictions. Note that both prediction methods use the same corrected parameter estimates from TMB, but the preferential model uses the mode of $[\mathbf{S} \mid \mathbf{Y}, \mathbf{X}]$ and the non-preferential model uses kriging. 


\section{Chapter 6}

\section{Preferential Sampling Analysis}

In this chapter we first conduct a real data example of preferential sampling in Section 6.1, in which we assess the predictive capability of the preferential model using TMB in comparison to standard methods for historical ozone data. We follow this with an assessment of TMB model performance across varying degrees of preferential sampling and sample sizes in Section 6.2.

\subsection{Ozone Data Analysis}

We conduct an analysis on ozone data obtained from the Air Quality System Data Mart internet database (AQS, 2018) available at http://www .epa. gov/ttn/airs/aqsdatamart. This data consists of air quality measurements obtained by the United States Environmental Protection Agency (EPA) at monitoring stations across the USA, where sampled values are 1hour averages. We consider the daily summary data from 2017 and analysed the mean daily ozone values for measuring stations in the domain $-120^{\circ}$ to $-90^{\circ}$ longitude and $35^{\circ}$ to $45^{\circ}$ latitude on March 31st 2017 . We wish to use this data to predict the ozone field on this date across the domain.

Before analysing the data, one should be aware of the stratospheretroposphere exchange of ozone (STE) phenomenon, which is a facet of the atmospheric circulation between the stratosphere and troposphere, which are two connected regions of the Earth's atmosphere (Mohanakumar, 2008). Typically, there is a maximum of STE during spring in the Northern Hemisphere, resulting potentially large ozone levels (Yang et al., 2016). Hence, the data we analyse in this chapter may not be representative of other months.

The full data set consists of 230 stations from which we artificially created both non-preferential (random) and preferential subsamples. To generate these data sets we randomly sampled 30 sites to be withheld for prediction assessment and then sampled 40 from the remaining 200 stations either randomly $(\beta=0)$ or preferentially $(\beta=100)$ according to the sampling inten- 


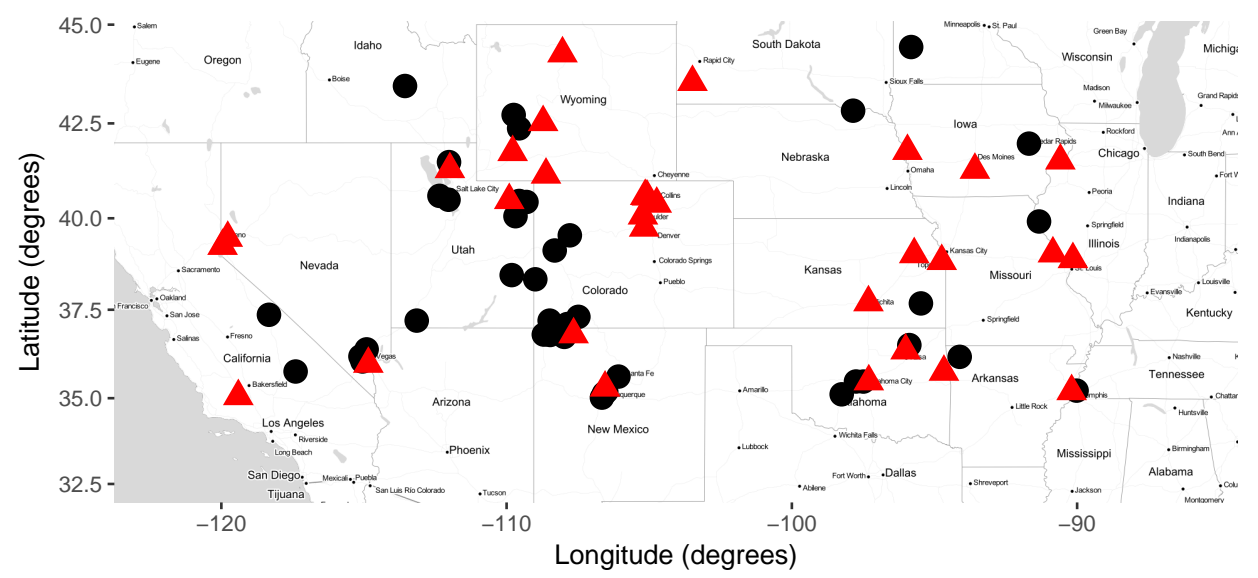

Figure 6.1: Example data set from the Ozone analysis. Black circles are monitoring sites in the example data set and red triangles reserved for prediction. Map tiles by Stamen Design, under CC BY 3.0. Data by OpenStreetMap, under ODbL.

sity (3.4), which implies that the preferentially chosen sites should typically contain larger ozone values than you would expect from a random sample. We assess random samples to quantify any possible inherent bias or preferential nature in the original data set. If there appears to be prediction bias even in our randomly sampled stations, then this might mean that there is a preferential effect already present in the data we are analysing. We repeated this 100 times (for both sampling systems) and fit both the non-preferential and TMB models. Figure 6.1 shows one example of a preferentially sampled data set, where the black circular points are the preferential subsample and the red triangles the sites used for prediction comparison. This procedure to illustrate the effect of preferential sampling on real data is similar to that used by Gelfand et al. (2012).

To analyse the non-preferential and preferential subsamples we assumed that the ozone field is a stationary Gaussian process with Matèrn covariance structure and constant mean, where we assumed the smoothness parameter is known as $\kappa=1$. For the standard prediction method we used the nonpreferential model to obtain parameter estimates and then used these as plug-in values for the ordinary kriging prediction across a $59 \times 19$ lattice. The standard predictions for the preferential data set shown in Figure 6.1 can seen in first panel of Figure 6.2. 
Next we fit the full preferential model in TMB to the same data. As in Chapter 5 we used the mode of $[\mathbf{S} \mid \mathbf{X}, \mathbf{Y}]$ obtained from TMB as our predictive density. The TMB prediction from the example data set shown in Figure 6.1 can be seen in the second panel of Figure 6.2. We represent the RMSPE at prediction sites in Figure 6.2 in terms of triangle size (larger triangle means larger RMSPE) and there was a clear reduction in error when using the preferential model in central test sites that were not located next to measurement sites. In this particular example the two predictive distributions were similar between $-110^{\circ}$ and $-105^{\circ}$ longitude which makes sense since this was the most densely sampled region. However, the preferential model detected the oversampling of high ozone areas and reduced the estimate for the constant mean of the field from $\hat{\mu}=0.037$ for the standard model to $\hat{\mu}=0.030$ in the preferential model; consequently the predictions tended towards different values. However there was a noticeable increase in the rate at which the ozone prediction decreased from the central high valued region in the preferential model compared to the standard method. This is in part due to the reduced range parameter estimate $(\hat{\phi}=1.61$ for the preferential model and $\hat{\phi}=1.89$ in the standard model), but also due to the preferential model identifying regions of sparse sampling being unlikely to have large ozone values. This corresponds to the estimate $\hat{\beta}=127.69$ which correctly identified that sites with larger ozone values were more likely to have been sampled. This was most evident in the region between longitudes $-105^{\circ}$ and $-100^{\circ}$ and the North-West where the preferential model clearly reduced the ozone prediction in comparison to the standard method. The prediction variances in the third and fourth panels of Figure 6.2 followed similar patterns across methods, but noticeably the preferential model had larger prediction variances in areas of sparse sampling.

We repeat the analysis previously discussed on the single example data set across 100 preferentially and 100 non-preferentially sampled data sets generated in the same manner. To assess model performance we compare various scoring functions for both models. We compare the bias, root-meansquare-prediction error (RMSPE) and mean ignorance score (MIGN) across all 30 test stations in each data set. The bias, RMSPE and MIGN for the 


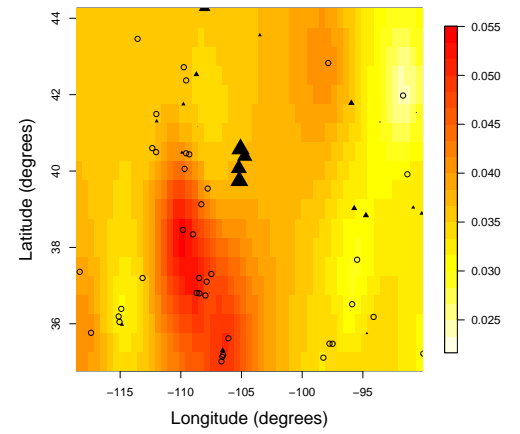

(a) Kriging Prediction

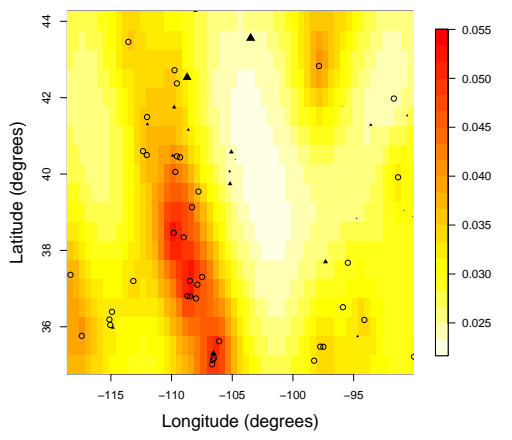

(b) Posterior Prediction

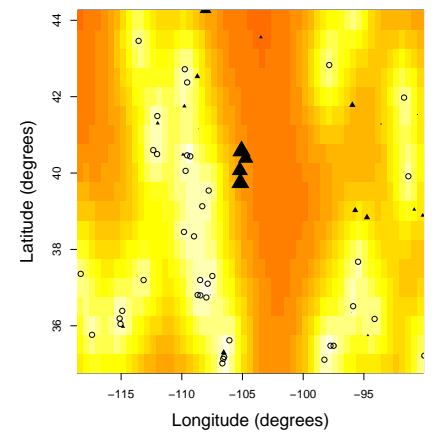

(c) Kriging Variance

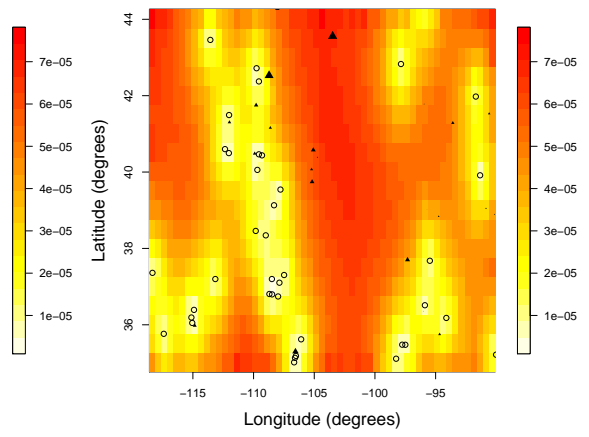

(d) Posterior Variance

Figure 6.2: Comparison of ozone prediction on the example data set between kriging using standard MLE plug-in parameters and the mode of $[\mathbf{S} \mid \mathbf{X}, \mathbf{Y}]$ obtained from TMB. Circles are the sampling locations and triangles the test sites with the size of each triangle the relative root-mean-square-predictionerror. 
$j$ th $(j=1, \ldots, 100)$ data set are defined as

$$
\begin{aligned}
\operatorname{RMSPE}_{j} & =\frac{1}{30} \sum_{k=1}^{30} \sqrt{\left(\hat{\mathbf{S}}_{j, i}-\mathbf{S}_{j, i}\right)^{2}} \\
\operatorname{Bias}_{j} & =\frac{1}{30} \sum_{i=1}^{30}\left(\hat{\mathbf{S}}_{j, i}-\mathbf{S}_{j, i}\right), \\
\operatorname{MIGN}_{j} & =\frac{1}{30} \sum_{i=1}^{30}\left(\frac{\left(\hat{\mathbf{S}}_{j, i}-\mathbf{S}_{j, i}\right)^{2}}{\sigma_{j, i}^{2}}+2 \log \left(\sigma_{j, i}^{2}\right)\right)
\end{aligned}
$$

where $\hat{\mathbf{S}}_{j, i}$ and $\mathbf{S}_{j, i}$ are the predicted and actual station value of the ozone field $\mathbf{S}$ at site $i$ in the $j$ th data set respectively and $\sigma_{j, i}^{2}$ the prediction variance at site $i$ in the $j$ th data set.

Figure 6.3 plots these scoring functions across both models and data sampling methods. The $x$-axis consists of the TMB and standard models with each panel plotting a different scoring function. Within the panels each plot compares a different data set type (non-preferentially and preferentially sampled) which is labeled as the title of the plot.

First we analyse the non-preferentially (randomly) sampled data set, which was included to assess whether there was a preferential effect inherent in the full data set. There was no evidence of prediction bias in this case, with the mean of $\operatorname{Bias}_{j}$ across all randomly sampled fields -0.00003 using the standard model and -0.0001 using TMB. For the preferential data sets there was a clear reduction in bias when using predictions from the preferential model in comparison to the standard model, which also translates to a decrease in RMSPE for the preferential model. In addition the final panel shows that the ignorance scores were generally superior for the preferential model, showing suitable prediction variance estimation.

Having said this, there is downside to the preferential model revealed when we compare the bias and RMSPE from the randomly sampled data sets. In this case the point predictions appeared slightly worse when using TMB, with greater variability in the bias and slightly larger RMSPE's. However, the ignorance scores appeared very similar, if not slightly improved when using the preferential model in TMB. The gains when using the preferential model in the preferential data set, particularly in bias and ignorance scores, appears greater than the drawbacks for using it on the non-preferential data sets. 


\subsection{Ozone Data Analysis}
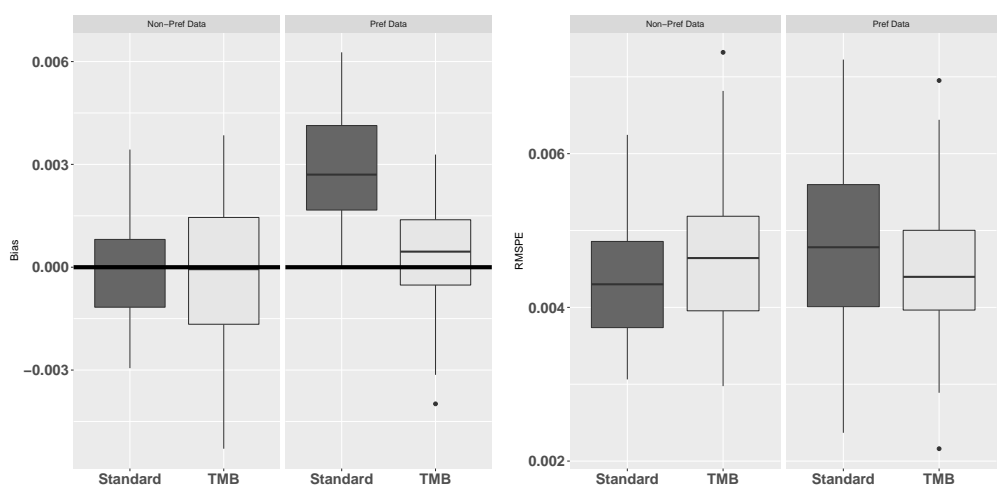

(a) Bias Comparison

(b) RMSPE Comparison

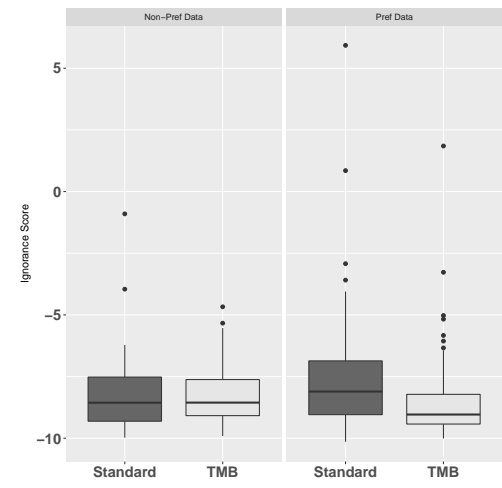

(c) Mean Ignorance Score Comparison

Figure 6.3: Comparison between scoring functions of kriging prediction using standard MLE plug-in parameters and the mode of $[\mathbf{S} \mid \mathbf{X}, \mathbf{Y}]$ obtained from TMB across 30 prediction locations. Smaller root-mean-square prediction errors and mean ignorance scores are considered better. 


\subsection{Comparison Between Various Degrees of Preferential Sampling}

In this section we compare parameter estimates and spatial prediction between the preferential TMB model and standard methods across varying degrees of preferential sampling and sample sizes. We compare how the effect of preferential sampling and attempted correction alters across 100 latent fields, generated using the same parameters as those in Section 4.5. We assess how the bias in parameter estimates and prediction change with varying values $\beta=0,0.5,1,1.5$ from the intensity function (3.4) across sampling sizes of $n=50,100,150,200$ for both the standard and TMB models. The aim here is to determine how various levels of preferential sampling impact spatial inference and to find out which sampling systems are most at risk. We would expect smaller values of $|\beta|$ and larger sample sizes to have smaller preferential effects, however to what degree this changes and which is more important will be analysed. As in previous sections, we compare the prediction bias between the mode of $[\mathbf{S} \mid \mathbf{Y}, \mathbf{X}]$ obtained from TMB and ordinary kriging using plug-in parameters from the standard model across a $31 \times 31$ $(N=961)$ dimensional lattice.

We first compare estimates of the mean parameter $\hat{\mu}$ obtained using the Laplace approximation of the full likelihood in TMB and a non-preferential likelihood. For each combination of sample size and preferential parameter we define the mean bias as

$$
\frac{1}{100} \sum_{j=1}^{100} \hat{\mu}_{j}-\mu
$$

where $\hat{\mu}_{j}$ is the mean parameter estimate in data set $j=1, \ldots, 100$ and $\mu$ is the true parameter, in our case $\mu=4$.

Figure 6.4 shows how biases of the mean parameter estimate vary across combinations of likelihood methods, preferential parameters $(\beta)$, and sample sizes. Each panel consists of results from a particular sample size (listed at the top of each plot) with $\beta$ varying across the $x$-axis and the colour of each boxplot the likelihood method used to obtain the estimate. Clearly, there was an increase in bias as $\beta$ got larger using the standard method across all sample sizes. This bias was reduced slightly in the larger sample sizes, which makes sense since larger sample sizes increase the likelihood of samples being taken across a wider spectrum of field values. Correction of this bias using the full likelihood in TMB was successful across all $\beta$ values. Interestingly, sample size had little effect on the bias variability across both 


\subsection{Comparison Between Various Degrees of Preferential Sampling}

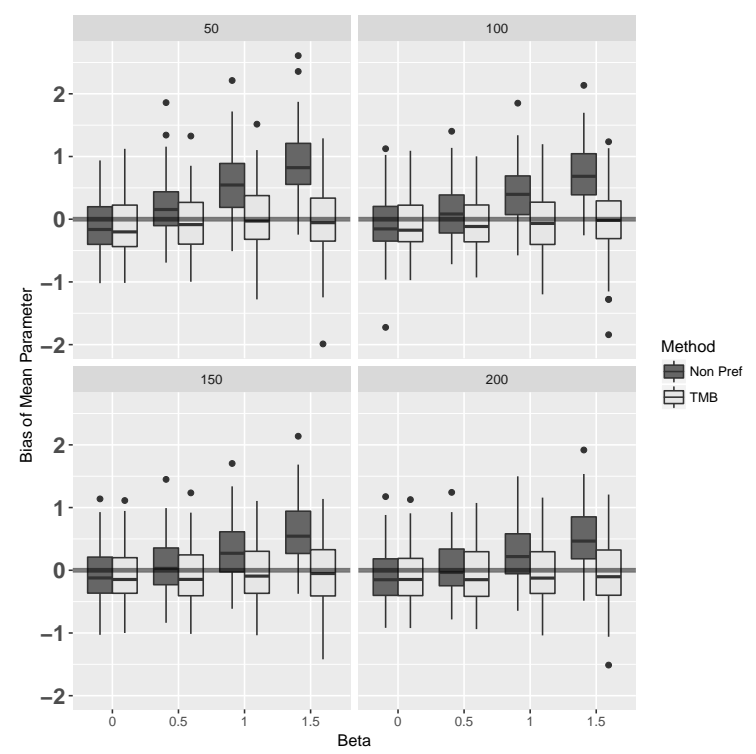

Figure 6.4: Comparison between bias of the mean parameter estimate $\hat{\mu}$ using Laplace approximation in TMB and a non-preferential likelihood.

methods, with the only noticeable effect being the reduction in standard MLE bias as $n$ increased for positive $\beta$ values.

Next we compare mean prediction bias for each combination of $\beta$ and sample size between the two methods. Assuming $N=961$ is the number of prediction points on the lattice, we define the mean bias for the $j$ th data set $(j=1, \ldots, 100)$ as

$$
\frac{1}{961} \sum_{i=1}^{961} \hat{\mathbf{S}}_{j, i}-\mathbf{S}_{j, i},
$$

where $\mathbf{S}_{j, i}$ and $\hat{\mathbf{S}}_{j, i}$ are the true and predicted values respectively at $\mathbf{x}_{i} \in$ $[0,1] \times[0,1]$ for the $j$ th field. We plot the mean prediction biases in Figure 6.5 using the same presentation as in Figure 6.4. In the case of ordinary kriging using non-corrected parameter plug-in values there was an increase in mean bias as $\beta$ increased, as we would expect. Here the impact of sample size appeared greater than in the parameter estimation case, with biases for $\beta=1$ and $n=50$ comparable to $\beta=1.5$ and $n=150$. This is likely due to the combination of increased sample sizes reducing parameter bias but also providing more prediction information as a consequence of simply having more sampled locations. When using the mode of $[\mathbf{S} \mid \mathbf{Y}, \mathbf{X}]$ there was a clear 


\subsection{Comparison Between Various Degrees of Preferential Sampling}

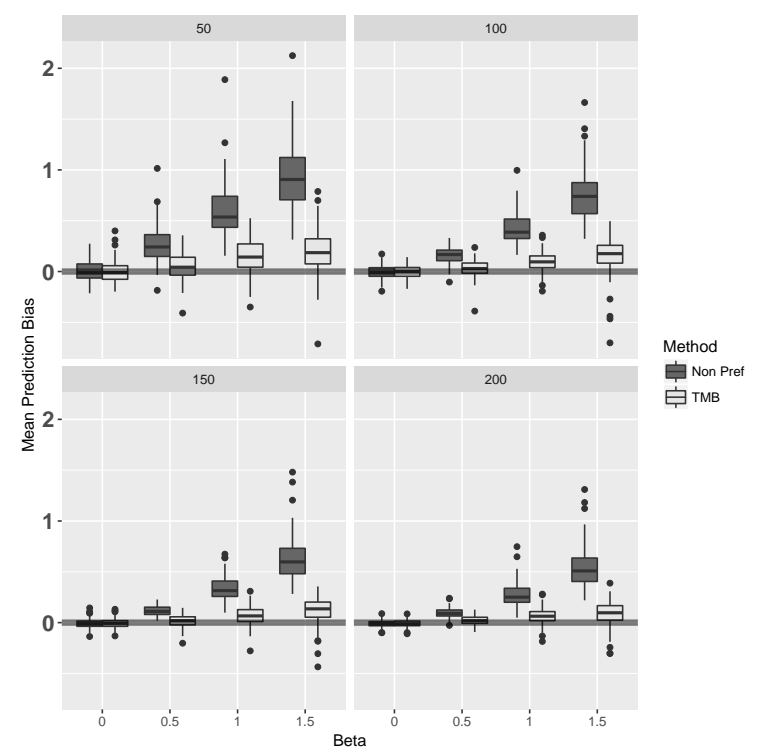

Figure 6.5: Comparison between prediction bias using mode of $[\mathbf{S} \mid \mathbf{Y}, \mathbf{X}]$ and ordinary kriging with plug-in parameters obtained from a non-preferential likelihood.

reduction in bias, but unlike the case of parameter correction, this bias was not fully eliminated. Interestingly the median bias did not appear to change across $n$ much, with the biggest change being the reduction in the variability of the mean bias as $n$ increased.

Finally we assess the ignorance scores. Ignorance scores were discussed in Section 5.3 and can be used to compare performance of the prediction methods whilst accounting for their uncertainty. We use the mean ignorance score (MIGN) defined in (5.5) to compare prediction on each data set between methods. This scoring function gives an average ignorance score across all prediction locations for each of the 100 data sets in each sample size and preferential parameter combination. We define the difference in MIGN for the $j$ th data set as,

$$
\mathrm{MIGN}_{j}^{\text {Diff }}=\mathrm{MIGN}_{j}^{\mathrm{P}}-\mathrm{MIGN}_{j}^{\mathrm{NP}},
$$

where $\mathrm{MIGN}_{j}^{\mathrm{P}}$ and $\mathrm{MIGN}_{j}^{\mathrm{NP}}$ are the MIGN for the preferential prediction model using the mode of $[\mathbf{S} \mid \mathbf{Y}, \mathbf{X}]$ and the non-preferential kriging model respectively. Therefore for a particular data set $j$, a negative value of $\mathrm{MIGN}_{j}^{\text {Diff }}$ means that the preferential model had a lower ignorance score (which means 


\subsection{Comparison Between Various Degrees of Preferential Sampling}

it performed better than the standard model) and a positive score that the preferential model had a larger ignorance score (which means that it performed worse than the standard model).

We plot MIGN ${ }^{\text {Diff }}$ for various combinations of sample size and preferentiability in Figure 6.6. The preferentiability parameter $\beta$ increases along the $x$-axis and boxplot colour represents sample size. As one would expect, as $\beta$ increased the values of MIGN ${ }^{\text {Diff }}$ tended to decrease. This means that as we increased the effect of preferential sampling, the difference between the ignorance scores across the two methods also increased. This makes sense when observing the difference in prediction bias from Figure 6.5 and confirms that prediction variances are also satisfactory. It also appears that as sample size increased the variability in MIGN ${ }^{\text {Diff }}$ decreased for all $\beta$. On the one hand this suggests that the difference in ignorance score is more consistent, however the variability is mostly contained to negative values of the score showing that the sample size was having much more of an impact on the ignorance score of the non-preferential model in comparison to the preferential model. Small sample sizes more regularly produce very large ignorance scores for the non-preferential model, with this impact much less noticeable for the preferential equivalent. Finally we note that as sample size increased, MIGN ${ }^{\text {Diff }}$ tended closer to 0 for positive values of $\beta$. This means that the difference in ignorance score between the two prediction methods decreased as sample size increased, which makes sense since we showed in Figures 6.4- 6.5 that bias was reduced at larger sample sizes. 


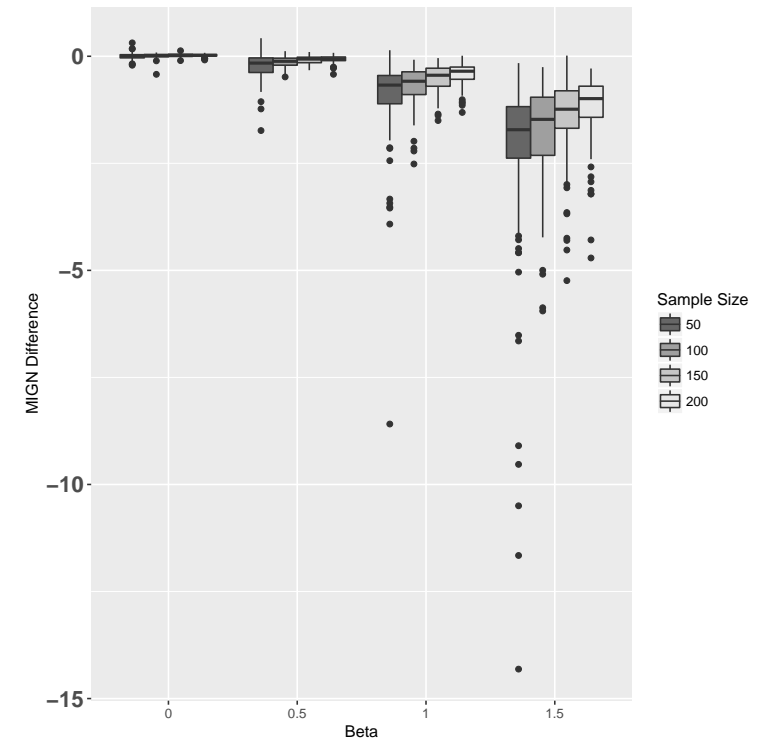

Figure 6.6: Comparison between mean ignornace score differences using mode of $[\mathbf{S} \mid \mathbf{Y}, \mathbf{X}]$ and ordinary kriging with plug-in parameters obtained from a non-preferential likelihood. 


\section{Chapter 7}

\section{Modelling Marine Mammal Movement}

In this chapter we first review marine mammal tagging in Section 7.1 and give a background on movement models in Section 7.2. We discuss extensions of these models such as introducing location measurement error using state space modelling in Section 7.2.1 and correlated random walks in Section 7.2.2. Finally in Section 7.2 .3 we discuss how to implement these models in TMB.

\subsection{Background on Marine Tagging}

Marine mammal tagging first began in the early 20th Century to record seal populations in the Bering Sea with hot iron branding of seals in 1923 and soon the replacement of this method with small aluminum tag attachment around St Paul island in 1927 (Scheffer et al., 1984). By the 1930s the National Institute of Oceanography (United Kingdom) began rudimentary tagging of blue, fin and humpback whales in the Antarctic (Mackintosh, 1952; Rayner, 1939). These initial forays into understanding the movement and migrations of whales used tubes of stainless steel marked with serial numbers and a notice of reward for their return, attached to the creatures using shotguns.

Soon techniques were developed for telemetry tagging via radio transmissions, with harnesses attached to California Gray Whales off the coast of Mexico (Norris and Gentry, 1974). Relative to whales, small marine mammals such as seals have proved easier to track, with tags glued to the upper back (Stewart et al., 1989). The use of satellites to track animals was first tested by Craighead et al. (1972) who successfully tracked elk movement for a one month period. By the 1980's the Argos System (http://www.argos-system.org/) was set up which could acquire location and environmental data from any location on earth (Fancy et al., 1988). This system has grown in scope ever since, with an increase from 
1200 to 8000 concurrent land and ocean based animals being tracked by the system between 1997 and 2011 (Douglas et al., 2012). In both these cases however, location data of the tagged mammals is not available underwater. Therefore tracking deals purely in surface locations, which depending on the species, may be infrequent.

Although Argos is an extremely valuable utility, it does have drawbacks. The main issue with the data is the variable accuracy of location measurements. Argos location measurements are assigned a score $(3,2,1, \mathrm{~A}, \mathrm{~B}, \mathrm{Z})$ where 3 is the highest quality measure (least measurement error), to $\mathrm{Z}$ being the worst quality. A,B and $\mathrm{Z}$ are not usually assigned error whilst Johnson et al. (2008) suggest removing class $\mathrm{Z}$ data altogether due to the high probability of outliers. Therefore, Argos data usually involves a substantial amount of pre-processing, with research into filtering techniques such as the speed-distance-angle-filter (Freitas et al., 2008) and Douglas Argosfilter (Douglas et al., 2012) developed to assess the credibility of movement speed and turning angles.

A more accurate alternative is to use the Global Positioning System (GPS) which provides more accurate location measurements and is common in land based tracking such as polar bears (Auger-Méthé et al., 2016) and various birds such as albatrosses (Weimerskirch et al., 2002) and gannets (Votier et al., 2010). However such systems are less appropriate for marine systems since GPS requires several seconds of exposure to obtain a location estimate (Dujon et al., 2014). Recently Fastloc-GPS tags (http: //www.wildtracker.com) have become more popular due to their improved accuracy compared to Argos and that these systems only require a fraction of a second to obtain a location estimate (Thomson et al., 2017). The downside to these tags however is their cost, which is much higher than Argos (Auger-Méthé et al., 2017).

\subsection{Modelling of Marine Mammal Movement}

Let us model the true location of a marine mammal at time $t$ with a stochastic process $\mathbf{X}(t)$, note that this does not depend on the depth of the animal, only the 2-dimensional spatial representation. This continuous process is observed in discrete time since the locations are only obtained when the animal is at the surface of the water. The time between observations can vary based on the species and current behavioral state of the animal.

Therefore marine mammal tracking data consists of location data $\mathbf{X}=$ $\left(\mathbf{X}\left(t_{1}\right), \ldots, \mathbf{X}\left(t_{n}\right)\right)$ where $t_{1}, \ldots, t_{n}$ are the sampling times which may or 
may not be equally spaced. Since the data is correlated, in that the location at $\mathbf{X}\left(t_{k}\right)$ depends on the location at $\mathbf{X}\left(t_{k-1}\right)$, stochastic differential equations (SDEs) have been commonly used to model the movement patterns of tagged marine mammals. Section 2.5 provides a brief background on SDEs. Since the data is obtained in discrete time, in general modelling the continuous SDE form in $(2.15)$ is conducted via the discrete Euler-Maruyama approximation (see, e.g. Brillinger et al. (2001)). In this case one can write

$$
\mathbf{X}\left(t_{k+1}\right)=\mathbf{X}\left(t_{k}\right)+\boldsymbol{\mu}\left(\mathbf{X}\left(t_{k}\right), \boldsymbol{\theta}\right)\left(t_{k+1}-t_{k}\right)+\boldsymbol{\Sigma}(\boldsymbol{\theta}) \sqrt{t_{k+1}-t_{k}} \mathbf{A}\left(t_{k+1}\right),
$$

where $\boldsymbol{\mu}: \mathbb{R}^{2} \rightarrow \mathbb{R}^{2}$ is the drift function and $\mathbf{A}\left(t_{k+1}\right)$ independent multivariate standard normal variables. Note that the drift function determines the expected movement direction and velocity at a particular location.

An early example of the SDE approach to animal movement modelling was by Dunn and Gipson (1977), who used multivariate Ornstein-Uhlenbec (OU) processes to model radio tag data from dear. The OU approach simplifies movement by specifying a home-range to which the animal is expected to return, which can be represented using a drift function resulting in attraction towards this home-range area. For example, using the OU model one could model attraction towards a home located at $\boldsymbol{\beta}=\left(\beta_{1}, \beta_{2}\right) \in \mathbb{R}^{2}$ using

$$
\mathrm{d} \mathbf{X}(t)=\alpha(\boldsymbol{\beta}-\mathbf{X}(t)) \mathrm{dt}+\sigma I_{2} \mathrm{~d} \boldsymbol{B}(t),
$$

where $\alpha \in \mathbb{R}, \sigma \in \mathbb{R}^{>0}, \boldsymbol{B}(t)$ is a Brownian process and $I_{2}$ is the $2 \times 2$ diagonal matrix. The coloured lines in the left panel of Figure 7.1 show examples of 6 tracks of 150 observations each simulated from the SDE in $(7.2)$ with $\alpha=$ 0.05 , home range location $\boldsymbol{\beta}=(100,-100)$ and $\sigma=2$. The time between each observation follows an exponential distribution with rate parameter $\lambda=10$. The black arrows direction and tail length represent direction and relative velocity of the expected movement $\boldsymbol{\mu}(\mathbf{X}(t), \boldsymbol{\theta})=\alpha(\boldsymbol{\beta}-\mathbf{X}(t))$ at that location, which can be viewed as the negative gradient of the potential function

$$
H(\mathbf{X}(t))=-\alpha\left(\beta_{1} X_{1}(t)+\beta_{2} X_{2}(t)\right)+\frac{\alpha}{2}\left(X_{1}(t)^{2}+X_{2}(t)^{2}\right),
$$

where $\mathbf{X}(t)=\left(X_{1}(t), X_{2}(t)\right)$. Note that we defined potential functions in Section 2.5. We plot this potential function in the right panel of Figure 7.1 and clearly movement down the gradient is towards the home zone $\boldsymbol{\beta}$.

Blackwell (1997) expanded these methods when modelling wood mouse movement to include binary behavioural states, which changed the diffusion process parameters upon switching. One example they discuss is an OU 
process when foraging with attraction towards areas of prey, whilst when traveling the model switches to a random walk. Various other early SDE approaches in ecology are summarised in Hallam and Levin (1986), however in these cases the models were restricted to terrestrial animals and birds, which are observable at more frequent and regular time intervals.
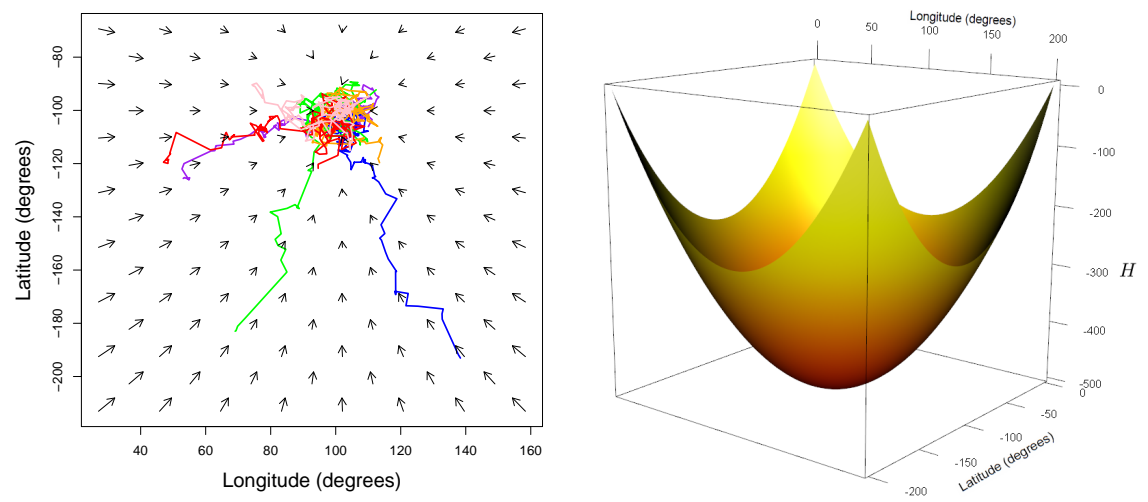

Figure 7.1: Left Panel: Example of 6 simulated tracks each consisting of 150 observations using an Ornstein-Uhlenbec process, with arrow direction and tail length showing direction and relative strength of drift function (expected movement) at that location. Right Panel: Potential function for the drift function used to generate the data in the left panel.

Much of the pioneering in animal movement SDE models was by David Brillinger, particularly with his modelling of marine mammal movement. Some notable examples include work on elephant seals (Brillinger et al., 2002 Brillinger and Stewart, 1998) and Hawaiian monk seals (Brillinger et al., 2006, 2008). Brillinger was particular interested in visualising movement through the potential field, such as the one in the right panel of Figure 7.1; a variety of models and discussions on this topic are available in Brillinger (2012). Brillinger et al. (2008) also considers various points of attraction in OU models to represent outbound movement from shore to forage locations, and then return movement back to shore. This work was similar to the behavioural state ideas of Blackwell (1997) but with a known switching time between states, where the states change the point of attraction. 


\subsubsection{State Space Movement Models}

So far in this chapter we have been assuming that the movement models contain no latent (unobserved) states. However to build models which reflect both the complex nature of movement persistence and the uncertainty in sampling locations, we need to consider estimation of latent states such as "true" sampling locations, behavioral states and underlying velocities in the animal movement. In this case state-space models (SSMs) have become increasingly popular in movement ecology with some notable literature including Auger-Méthé et al. (2016); Buckland et al. (2004); Patterson et al. (2008). SSMs account for multiple sources of variation, the variation of the process (for example the movement process $\mathbf{X}$ ) and observation error (Newman et al., 2014). We define a SSM as a set of coupled equations

$$
\begin{aligned}
& \mathbf{Z}\left(t_{k+1}\right)=h\left(\mathbf{X}\left(t_{k+1}\right), \boldsymbol{\epsilon}\left(t_{k+1}\right), \boldsymbol{\theta}\right), \\
& \mathbf{X}\left(t_{t+1}\right)=f\left(\mathbf{X}\left(t_{k}\right), \boldsymbol{\xi}\left(t_{k+1}\right), \boldsymbol{\theta}\right),
\end{aligned}
$$

where $\mathbf{Z}=\left(\mathbf{Z}\left(t_{1}\right), \ldots, \mathbf{Z}\left(t_{n}\right)\right)$ are discretely observed data at times $t_{1}, \ldots, t_{n}$ which themselves are a function of some latent process $\mathbf{X}(t)$. Sometimes SSMs are referred to as hidden Markov models (HMMs) (Cappé et al., 2005), although we follow Patterson et al. (2017) in labeling cases in which there are a finite number of possible latent states as HMMs, and when the underlying latent process is continuous and can therefore involve an infinite number of states as SSMs. We can also view HMMs as a special subset SSMs which are less flexible but also less computationally challenging (Langrock et al., 2012).

Consider the case in which we no longer assume that we know true sampling locations, since the coordinates provided by a tag have some level of observation error. The magnitude of this error depends on multiple factors. One is the type of satellite system used by the tag. Argos based tags are cheaper and more convenient to implement that GPS but also have larger measurement errors (see Section 7.1). In this case we might assume that the data we obtain consists of observed locations $\mathbf{Z}=\left(\mathbf{Z}\left(t_{1}\right), \ldots, \mathbf{Z}\left(t_{n}\right)\right)$ which are the unobservable true locations, denoted $\mathbf{X}=\left(\mathbf{X}\left(t_{1}\right), \ldots, \mathbf{X}\left(t_{n}\right)\right)$, measured with some error. If we model the true locations as a continuous latent process with observations in discrete time using the discretised SDE from $(7.1)$, then we could write the SSM of the form from $(7.3)$ as

$$
\begin{aligned}
\mathbf{Z}\left(t_{k+1}\right) & =\mathbf{X}\left(t_{k+1}\right)+\boldsymbol{\epsilon}\left(t_{k+1}, \boldsymbol{\theta}\right), \\
\mathbf{X}\left(t_{k+1}\right) & =\mathbf{X}\left(t_{k}\right)+\boldsymbol{\mu}\left(\mathbf{X}\left(t_{k}\right), \boldsymbol{\theta}\right)\left(t_{k+1}-t_{k}\right)+\boldsymbol{\Sigma}(\boldsymbol{\theta}) \sqrt{t_{k+1}-t_{k}} \mathbf{A}\left(t_{k+1}\right),
\end{aligned}
$$


where $\boldsymbol{\epsilon}\left(t_{k+1}, \boldsymbol{\theta}\right)$ is some error distribution depending on the vector of model parameters $\boldsymbol{\theta}, \mathbf{X}\left(t_{k+1}\right)$ is the true location at time $t_{k+1}$ and $\mathbf{Z}\left(t_{k+1}\right)$ the observed location.

\section{Kalman Filtering}

There are various options for fitting SSMs and evaluating the subsequent likelihood function. A classic approach is to use the Kalman filter (KF) (Kalman, 1960) which relies on both processes being linear (ie/ $h(\cdot)$ and $f(\cdot)$ from $(7.3)$ are linear functions) and the error processes being Gaussian. The linearity constraints are lifted in the extended Kalman filter (EKF) (Jørgensen, 2007; Kristensen et al., 2004; Ribeiro, 2004) which allows for non-linear, but differentiable functions.

The KF methods use the fact that we can write the likelihood as the product of conditional densities. Denoting $\mathbf{Z}\left(t_{1: k}\right)=\left(\mathbf{Z}\left(t_{1}\right), \ldots, \mathbf{Z}\left(t_{k}\right)\right)$, we can write the likelihood function as

$$
L(\boldsymbol{\theta})=\left(\prod_{k=1}^{n-1}\left[\mathbf{Z}\left(t_{k+1}\right) \mid \mathbf{Z}\left(t_{1: k}\right)\right]\right)\left[\mathbf{Z}\left(t_{1}\right)\right]
$$

where if the error terms do not depend on the state and the observational noise is additive, then the conditional densities $\left[\mathbf{Z}\left(t_{k+1}\right) \mid \mathbf{Z}\left(t_{1: k}\right), \boldsymbol{\theta}\right]$ can be assumed Gaussian (Møller et al., 2011).

Therefore one may rewrite this likelihood as,

$$
L(\boldsymbol{\theta})=\left(\prod_{k=1}^{n-1} \frac{\exp \left\{-\frac{1}{2} \boldsymbol{\psi}_{k+1}^{T}(\boldsymbol{\theta}) \boldsymbol{R}_{k+1 \mid k}^{-1}(\boldsymbol{\theta}) \boldsymbol{\psi}_{k+1}(\boldsymbol{\theta})\right\}}{\sqrt{\operatorname{det}\left(\boldsymbol{R}_{k+1 \mid k}(\boldsymbol{\theta})\right)} 2 \pi}\right)\left[\mathbf{Z}\left(t_{1}\right)\right],
$$

where $\boldsymbol{R}_{k+1 \mid k}=\operatorname{Cov}\left(\mathbf{Z}\left(t_{k+1}\right) \mid \mathbf{Z}\left(t_{1: k}\right), \boldsymbol{\theta}\right)$ is the prediction covariance and $\boldsymbol{\psi}_{k+1}=\mathbf{Z}\left(t_{k+1}\right)-\mathbb{E}\left[\mathbf{Z}\left(t_{k+1}\right) \mid \mathbf{Z}\left(t_{1: k}\right), \boldsymbol{\theta}\right]$ the prediction error.

The likelihood can then be evaluated by conditioning on $\mathbf{Z}\left(t_{1}\right)$ and using the KF to recursively estimate both $\boldsymbol{R}_{k+1 \mid k}$ and $\boldsymbol{\psi}_{k+1}$ for $k=1, \ldots, n-1$. Details on the EKF estimation approach can be found in Appendix B.

\section{Laplace Approximation}

An alternative approach becoming ever more popular in animal movement problems is using Laplace approximations for SSMs (Albertsen et al., 2015; Patterson et al., 2017), implemented via AD Model Builder (ADMB) (Fournier et al., 2012) or Template Model Builder (TMB) (Kristensen et al., 2016) - see Section 4.4 for previous introduction on Laplace approximations and TMB. 
The advantage of using the Laplace approximation method for SSMs is similar to the advantage in the case of the preferential sampling, in that we can use a hierarchical framework to specify densities of our states conditional on the latent process. In addition, various assumptions required for the $\mathrm{KF}$ methods are not required for Laplace approximations, such as Gaussian errors. This is a particularly useful feature since, for example, Jonsen et al. (2005) claim that the $t$-distribution is more appropriate than Gaussian for modelling Argos tag location errors.

If we consider the SSM from (7.3) then the likelihood can be written in terms of the joint density of the observations $(\mathbf{Z})$ and latent states $(\mathbf{X})$ as

$$
L(\boldsymbol{\theta})=[\mathbf{Z}]=\int[\mathbf{Z}, \mathbf{X}] \mathrm{d} \mathbf{X}=\int[\mathbf{Z} \mid \mathbf{X}][\mathbf{X}] \mathrm{d} \mathbf{X} .
$$

The final factorisation uses densities of distributions which are usually much easier to specify than that of the conditional densities used in KFs, although it should be noted that the dimension of the integral grows with the number of observations $n$. In the case of the SSM example in (7.4) we would have

$$
\begin{aligned}
{[\mathbf{Z} \mid \mathbf{X}] } & =\left(\prod_{k=1}^{n-1}\left[\mathbf{Z}\left(t_{k+1}\right) \mid \mathbf{Z}\left(t_{1: k}\right), \mathbf{X}\right]\right)\left[\mathbf{Z}\left(t_{1}\right) \mid \mathbf{X}\right], \\
{[\mathbf{X}] } & =\left(\prod_{k=1}^{n-1}\left[\mathbf{X}\left(t_{k+1}\right) \mid \mathbf{X}\left(t_{1: k}\right)\right]\right)\left[\mathbf{X}\left(t_{1}\right)\right],
\end{aligned}
$$

where $\left[\mathbf{Z}\left(t_{k+1}\right) \mid \mathbf{Z}\left(t_{1: k}\right), \mathbf{X}\right]$ and $\left[\mathbf{X}\left(t_{k+1}\right) \mid \mathbf{X}\left(t_{1: k}\right)\right]$ follow the coupled equations (7.4) directly. Using the Laplace approximation methods implemented via TMB described in Section 4.4, we would then simply need to specify $-\log ([\mathbf{Z} \mid \mathbf{X}][\mathbf{X}])$ to evaluate the likelihood function. Furthermore, this method provides latent state estimates in the form of the mode of $[\mathbf{X} \mid \mathbf{Z}]$, a side effect of the Laplace method.

Although we do not extensively investigate the state-space framework for location measurement error in this thesis, it is important to be aware of these models and the various techniques used to fit them. We will be utilising the state-space framework for other latent processes obtained from tagged marine mammals in future sections using TMB, but incorporating some type of location error would be valuable future research. Throughout the remainder of this thesis we will be assuming that there is no measurement error in the models we propose. Notation of $\mathbf{X}$ will refer to the sampling locations which are assumed to be measured without error. 


\subsubsection{Correlated Random Walk Models}

The models from (7.1) and (7.2) describe movement directly through the tag locations, with the movement processes modelling the location $\mathbf{X}\left(t_{k+1}\right)$ as some function of $\mathbf{X}\left(t_{k}\right)$. However, Jonsen et al. (2005) proposed a more natural approach to describe animal movement via a correlated random walk (CRW) which models the differences in consecutive locations, rather than the locations themselves. This incorporates an inertia retaining similar movement tendency across successive times. Jonsen et al. (2005) defined a first-difference correlated random walk model (DCRW) for observations taken at equal time spacing to be

$$
\mathbf{X}(t+1)=\mathbf{X}(t)+\gamma \mathbf{T}(\theta)(\mathbf{X}(t)-\mathbf{X}(t-1))+\boldsymbol{\Sigma}_{\mathbf{X}} \mathbf{A}_{t+1}
$$

where $0 \leq \gamma \leq 1$ is a correlation parameter, $\boldsymbol{\Sigma}_{\mathbf{X}}$ a covariance matrix, $\mathbf{A}_{t}$ 's standard multivariate normal variables and $\mathbf{T}$ is a transition matrix to describe rotation of the correlated movement

$$
\mathbf{T}(\theta)=\left(\begin{array}{cc}
\cos (\theta) & -\sin (\theta) \\
\sin (\theta) & \cos (\theta)
\end{array}\right) .
$$

In this case $\theta$ is the mean turning angle. When $\gamma=0$ in $(7.6)$ we have a random walk, similar to the models in the previous section, whilst $0<\gamma<1$ varies the correlation in velocity and direction. If the data was observed at unequal time steps then this model can be adjusted to

$$
\begin{array}{r}
\mathbf{X}\left(t_{k+1}\right)=\mathbf{X}\left(t_{k}\right)+\gamma \mathbf{T}(\theta) \frac{\mathbf{X}\left(t_{k}\right)-\mathbf{X}\left(t_{k-1}\right)}{t_{k}-t_{k-1}}\left(t_{k+1}-t_{k}\right)+ \\
\boldsymbol{\Sigma}_{\mathbf{X}} \sqrt{t_{k+1}-t_{k}} \mathbf{A}_{t_{k+1}} .
\end{array}
$$

Notice that unlike the discrete approximation to a SDE defined in (7.1), the DCRW is not Markovian. A stochastic process $\left\{\mathbf{X}_{1}, \ldots, \mathbf{X}_{n}\right\}$ has the Markov property if for all $k \leq n$

$$
\left[\mathbf{X}_{k}=\mathbf{x}_{k} \mid \mathbf{X}_{k-1}=\mathbf{x}_{k-1}, \ldots, \mathbf{X}_{1}=\mathbf{x}_{1}\right]=\left[\mathbf{X}_{k}=\mathbf{x}_{k} \mid \mathbf{X}_{k-1}=\mathbf{x}_{k-1}\right] .
$$

In other words, the process is memoryless. However, in (7.7) notice that $\mathbf{X}\left(t_{k+1}\right)$ depends on both $\mathbf{X}\left(t_{k}\right)$ and $\mathbf{X}\left(t_{k-1}\right)$, hence we lose this Markov property. This is the reason why by using this model we can induce correlated movement, but with the side effect that the location at a given time is not just dependent on the last location but all previous locations.

To give an example of how the DCRW in (7.6) models movement, we simulate 3 tracks of 150 observations each using this model in Figure 7.2 . 
All tracks are initialised with the first two observations of $(0,0)$ and $(1$, 5) with the purple, green and red tracks corresponding to $\gamma=0,0.5$ and 0.8 respectively. We set the covariance matrix $\boldsymbol{\Sigma}_{\mathbf{X}}=I_{2}$ and mean turning parameter $\theta=0$ so that there is no expected turning angle in the movement, with the correlation entirely depending on the previous two locations. These simulations show the variety of movement that may be captured with these simple models. When $\gamma=0.5$ and 0.8 the movement includes periods of continuous travel in certain directions, but also areas similar to the random walk. When $\gamma=0$ movement is always a random walk which can be seen in the lack of directional persistence.

This model can be developed further by considering behavioural states. One example is to use the CRW or DCRW models but for which parameters are indexed by the behavioural state (Breed et al., 2009; Jonsen et al., 2005). This could be used to switch between periods of travel and foraging say, which in the example from Figure 7.2 might be to alter $\gamma$ to a smaller value when foraging in comparison to the travelling state.

An alternative to the DCRW was developed by Johnson et al. (2008), with the introduction of the continuous-time correlated random walk model (CTCRW), where instead of modelling the difference in locations we model the velocity in movement. Consider the continuous path $\mathbf{X}(t)$, then we can write the SDE

$$
\mathrm{d} \mathbf{X}(t)=\mathbf{v}(t) \mathrm{d} t+\sigma_{\mathbf{X}} I_{2} \mathrm{~d} \boldsymbol{A}(t),
$$

where $\sigma_{\mathbf{x}} \in \mathbb{R}^{>0}, \boldsymbol{A}(t)$ are bivariate standard normals and $\mathbf{v}(t)$ is the velocity, or in other words the instantaneous rate of change.

Johnson et al. (2008) suggest modelling the velocity as an OU process from (7.2). Take for example

$$
\mathrm{d} \mathbf{v}(t)=\alpha(\boldsymbol{\beta}-\mathbf{v}(t)) \mathrm{d} t+\sigma_{\mathbf{v}} I_{2} \mathrm{~d} \boldsymbol{B}(t)
$$

then the mean velocity is given by $\boldsymbol{\beta}=\left(\beta_{1}, \beta_{2}\right) \in \mathbb{R}^{2}$ where $\alpha \in \mathbb{R}$ controls the rate of change towards this mean velocity and $\sigma_{\mathbf{v}} \in \mathbb{R}^{>0}$ controls the variability in the velocity.

If we consider a discrete vector of our sampling observations $\mathbf{X}=$ $\left(\mathbf{X}\left(t_{1}\right), \ldots, \mathbf{X}\left(t_{n}\right)\right)$ and velocities $\mathbf{v}=\left(\mathbf{v}\left(t_{1}\right), \ldots, \mathbf{v}\left(t_{n}\right)\right)$ taken at not necessarily equal time points $\mathbf{t}=\left(t_{1}, \ldots, t_{n}\right)$, then we can write our likelihood

$$
L(\boldsymbol{\theta})=[\mathbf{X}]=\int[\mathbf{X}, \mathbf{v}] \mathrm{d} \mathbf{v}=\int[\mathbf{X} \mid \mathbf{v}][\mathbf{v}] \mathrm{d} \mathbf{v} .
$$




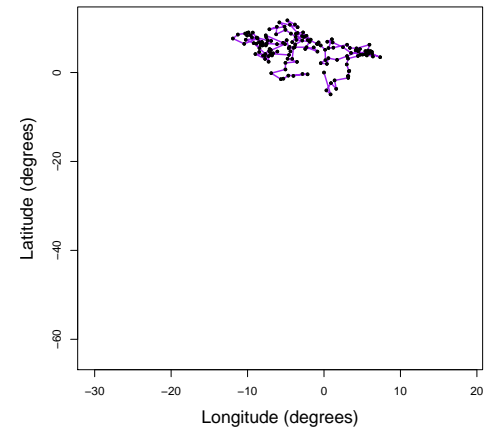

(a) $\gamma=0$

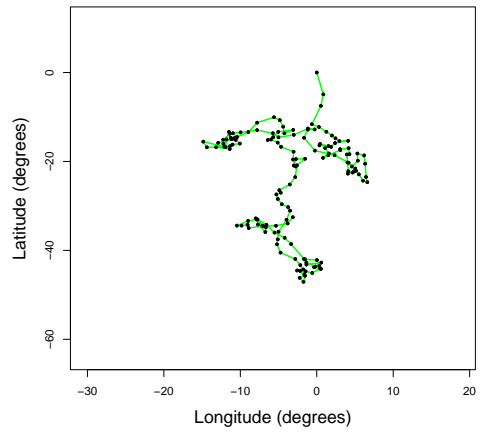

(b) $\gamma=0.5$

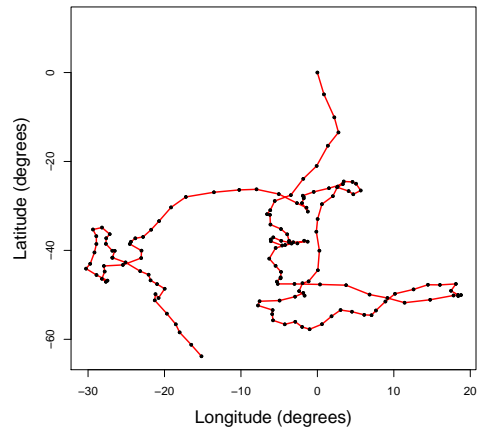

(c) $\gamma=0.8$

Figure 7.2: Example of 3 simulated tracks each consisting of 150 observations using first-difference correlated random walk from $(7.6)$. All tracks are initalised with the first two observations of $(0,0)$ and $(1,-5)$ with the purple, green and red tracks corresponding to $\gamma=0,0.5$ and 0.8 respectively. 
We can factorise both $[\mathbf{X} \mid \mathbf{v}]$ and $[\mathbf{v}]$ as

$$
\begin{aligned}
{[\mathbf{X} \mid \mathbf{v}] } & =\left[\mathbf{X}\left(t_{n}\right) \mid \mathbf{v}, \mathbf{X}\left(t_{(n-1)}\right)\right] \cdots\left[\mathbf{X}\left(t_{3}\right) \mid \mathbf{v}, \mathbf{X}\left(t_{2}\right)\right]\left[\mathbf{X}\left(t_{1}\right) \mid \mathbf{v}\right] \\
{[\mathbf{v}] } & =\left[\mathbf{v}\left(t_{n}\right) \mid \mathbf{v}\left(t_{(n-1)}\right)\right] \cdots\left[\mathbf{v}\left(t_{2}\right) \mid \mathbf{v}\left(t_{1}\right)\right]\left[\mathbf{v}\left(t_{1}\right)\right]
\end{aligned}
$$

where

$$
\begin{aligned}
\mathbf{X}\left(t_{k+1}\right) \mid \mathbf{v}, \mathbf{X}\left(t_{k}\right) & \sim N_{2}\left(\mathbf{X}\left(t_{k}\right)+\mathbf{v}\left(t_{k}\right)\left(t_{k+1}-t_{k}\right), \Sigma_{\mathbf{X}} \sqrt{t_{k+1}-t_{k}}\right) \\
\mathbf{v}\left(t_{k+1}\right) \mid \mathbf{v}\left(t_{k}\right) & \sim N_{2}\left(\mathbf{v}\left(t_{k}\right)+\alpha\left(\boldsymbol{\beta}-\mathbf{v}\left(t_{k}\right)\right)\left(t_{k+1}-t_{k}\right), \sigma_{\mathbf{v}} I_{2} \sqrt{t_{k+1}-t_{k}}\right) .
\end{aligned}
$$

Since the velocity is a continuous latent state we can therefore use the Laplace approximation implementation in TMB to estimate these states, which again provides estimates of the latent velocity states in the form of the mode of $[\mathbf{v} \mid \mathbf{X}]$.

To give an example of this, we simulate 3 tracks of 100 observations each using the CTCRW model from (7.8)-(7.9), where the time taken between observations is always 1 (ie/ $\left.t_{1}=1, \ldots, t_{100}=100\right)$. Figure 7.3 shows the 3 tracks which are all initialised with the first two observations of $(0,0)$ and $(1,1)$. The mean velocity is North-Easterly $\boldsymbol{\beta}=c(1,1)$ with the purple, green and red tracks corresponding to $\alpha=0.2,0.5$ and 1 respectively. We set the diffusion parameters $\sigma_{\mathbf{X}}=0.1$ and $\sigma_{\mathbf{v}}=2$ for all tracks. The black diagonal line shows the expected mean velocity direction towards the NorthEast. Here the tracks maintain correlation through the velocity which tends towards $\boldsymbol{\beta}=c(1,1)$ to varying extents. For both $\alpha=0.5$ (Panel (b)) and $\alpha=1$ (Panel (c)) it clear that the velocities are roughly North-Easterly in general, which induces correlated movement towards the North-East along the black diagonal line. However when $\alpha=0.2$ (Panel (a)) the tendency towards the mean velocity is greatly reduced. Over time one would expect the movement in this case to be North-Easterly, but with far more variation in the direction than the other 2 cases.

We use TMB to fit the CTCRW model to the first track only (purple track in Panel (a) of Figure 7.3 with $\alpha=0.2$ ), with Panels (a) and (b) in Figure 7.4 showing the latent velocity states in longitude and latitudinal directions respectively for 50 time points $t=25, \ldots, 75$. Here the black line is the true latent velocity and the red line the estimates along with $95 \%$ confidence intervals given by the shaded region. These confidence regions were calculated as the latent state estimates \pm 2 standard errors, which are calculated in TMB using the Hessian of the log-likelihood. The part of the track which these velocities represent is shown in Panel (c) of Figure 7.4. 


\subsection{Modelling of Marine Mammal Movement}

Clearly the latent states are estimated well, with the true states always contained within the confidence region.

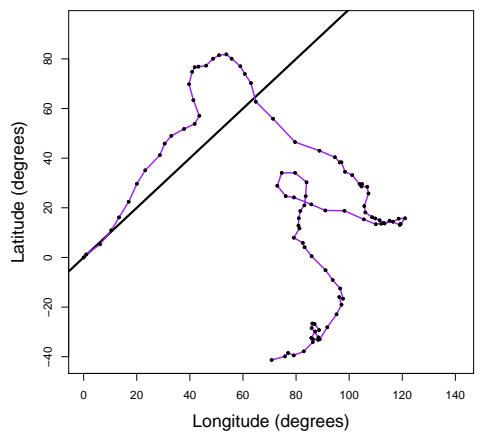

(a) $\alpha=0.2$

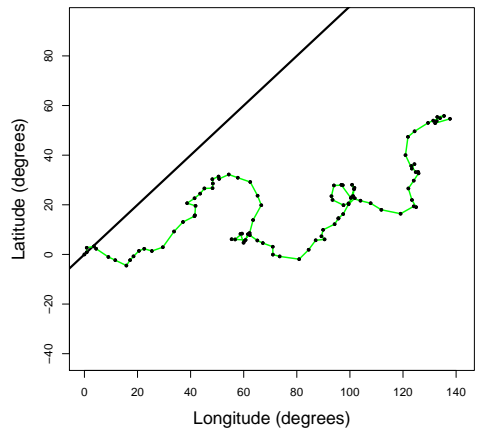

(b) $\alpha=0.5$

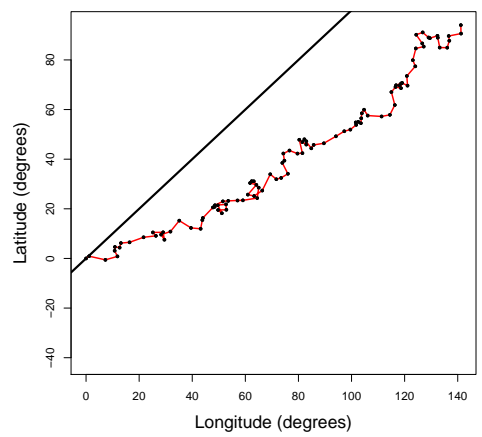

(c) $\alpha=1$

Figure 7.3: Example of 3 simulated tracks each consisting of 100 observations using the continuous-time correlated random walk model from $(7.8)-(7.9)$. The purple, green and red tracks correspond to $\alpha=0.2,0.5$ and 1 respectively. The black diagonal line shows the mean velocity direction $(\boldsymbol{\beta})$, which is heading in a North-Easterly direction. 


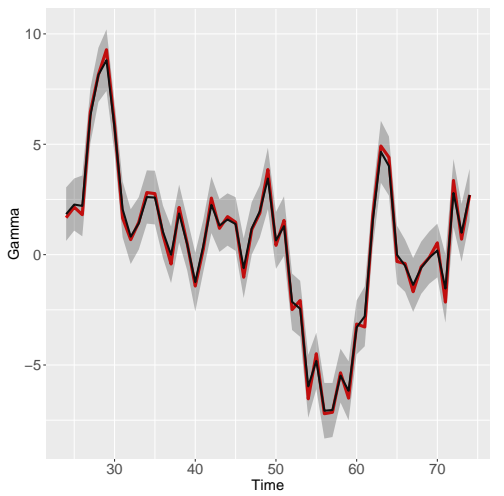

(a) Longitude Velocity

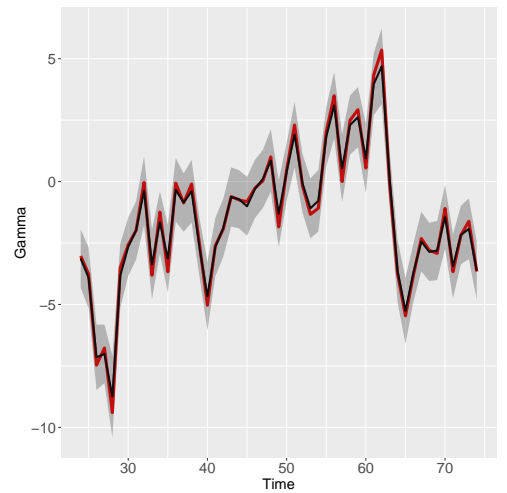

(b) Latitude Velocity

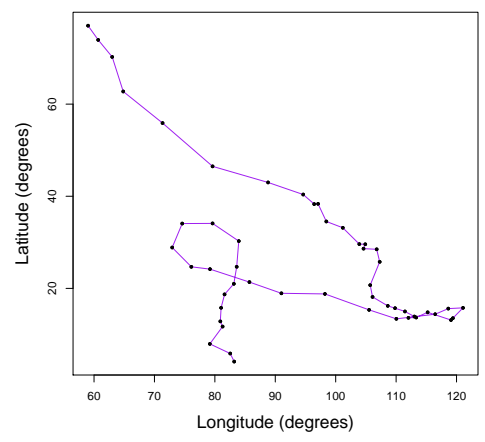

(c) Velocity Track Snippet

Figure 7.4: Panels (a)-(b) True latent longitudinal and latitudinal velocities for the $\alpha=0.2$ (purple) track between times 25-75 given in red. The black line and shaded region correspond to state estimates and $95 \%$ confidence region. Panel (c) Section of the track between times 25-75 corresponding to the latent states.

\subsubsection{Modelling Movement Using Template Model Builder (TMB)}

Since we will be implementing our movement models using TMB, we must make sure the likelihood is differentiable for the Laplace approximation (AugerMéthé et al., 2017; Bolker et al., 2013 Fournier et al., 2012). In the case of a CTCRW latent velocity state as described in the previous section, the differentiability property holds due to the continuity of the velocity in both latitudinal and longitudinal directions. However, in the case of behavioural 
states which might determine correlation parameters such as $\gamma$ in $(7.6)$ and $\alpha$ in (7.9), we must be careful. A discrete set of latent states will invalidate the differentiability of the likelihood and therefore not be implementable in TMB. One option is to estimate the behavioural states outside of TMB once the movement parameters have been estimated (Whoriskey et al., 2017), but we follow the approach of Auger-Méthé et al. (2017) who propose using a continuous behavioural state DCRW model similar to that in (7.7), which we can fully implement within TMB. This model is defined

$$
\begin{aligned}
\mathbf{X}\left(t_{k+1}\right) & =\mathbf{X}\left(t_{k}\right)+\gamma\left(t_{k}\right) \frac{\mathbf{X}\left(t_{k}\right)-\mathbf{X}\left(t_{k-1}\right)}{t_{k}-t_{k-1}}\left(t_{k+1}-t_{k}\right)+\Sigma_{\mathbf{X}}(\boldsymbol{\theta}) \sqrt{t_{k+1}-t_{k}} \mathbf{A}_{t_{k+1}}, \\
\gamma\left(t_{k+1}\right) & =\gamma\left(t_{k}\right)+\sigma_{\gamma}(\boldsymbol{\theta}) \sqrt{t_{k+1}-t_{k}} B_{t_{k+1}}
\end{aligned}
$$

where $\mathbf{A}_{t_{k+1}}$ and $B_{t_{k+1}}$ are 2 and 1-dimensional independent standard multivariate normal variables respectively, $\Sigma_{\mathbf{X}}(\boldsymbol{\theta})$ and $\sigma_{\gamma}(\boldsymbol{\theta})$ are $2 \times 2$ covariance matrices and 1-dimensional variance functions respectively and $\boldsymbol{\theta}$ is the full set of model parameters.

The model in (7.11) retains the DCRW structure of modelling first differences but includes both behavioural states and irregular sampling times. Considering the discretised SDE in (7.1) we can view (7.11) as having a drift function

$$
\boldsymbol{\mu}\left(\mathbf{X}\left(t_{1: k}\right), \mathbf{t}_{1: k}, \gamma\left(t_{k}\right), \boldsymbol{\theta}\right)=\gamma\left(t_{k}\right) \frac{\mathbf{X}\left(t_{k}\right)-\mathbf{X}\left(t_{k-1}\right)}{t_{k}-t_{k-1}},
$$

where $\mathbf{X}\left(t_{1: k}\right)=\left(\mathbf{X}\left(t_{1}\right), \ldots, \mathbf{X}\left(t_{k}\right)\right)$ and $\mathbf{t}_{1: k}=\left(t_{1}, \ldots, t_{k}\right)$. In this case, expected movement is towards a standardised difference in movement between the previous 2 observations, which can be thought of as a non-latent version of the CTCRW model where $\left(\mathbf{X}\left(t_{k}\right)-\mathbf{X}\left(t_{k-1}\right)\right) /\left(t_{k}-t_{k-1}\right)$ is an approximate velocity. The random walk $\gamma(t)$ determines the rate of correlation in the movement, where $\gamma(t)=0$ results in a random walk for $\mathbf{X}(t)$ and $0<\gamma(t) \leq 1$ a correlated movement model.

To fit a model of the form in (7.11) using TMB we can specify the likelihood of the sampling locations $\mathbf{X}=\left(\mathbf{X}\left(t_{1}\right), \ldots, \mathbf{X}\left(t_{n}\right)\right)$ as

$$
L(\boldsymbol{\theta})=[\mathbf{X}]=\int[\mathbf{X}, \boldsymbol{\gamma}] \mathrm{d} \boldsymbol{\gamma}=\int[\mathbf{X} \mid \boldsymbol{\gamma}][\boldsymbol{\gamma}] \mathrm{d} \boldsymbol{\gamma},
$$

where $\gamma=\left(\gamma\left(t_{1}\right), \ldots, \gamma\left(t_{n}\right)\right)$ are the latent behavioural states. We can then apply the Laplace approximation as described in Section 4.4.1 to obtain behavioural state estimates, since we can factorise both $[\mathbf{X} \mid \gamma]$ and $[\gamma]$ as

$$
\begin{aligned}
{[\mathbf{X} \mid \gamma] } & =\left[\mathbf{X}\left(t_{n}\right) \mid \boldsymbol{\gamma}, \mathbf{X}\left(t_{1:(n-1)}\right)\right] \cdots\left[\mathbf{X}\left(t_{3}\right) \mid \boldsymbol{\gamma}, \mathbf{X}\left(t_{1: 2}\right)\right]\left[\mathbf{X}\left(t_{1: 2}\right) \mid \gamma\right] \\
{[\gamma] } & =\left[\gamma\left(t_{n}\right) \mid \gamma\left(t_{1:(n-1)}\right)\right] \cdots\left[\gamma\left(t_{2}\right) \mid \gamma\left(t_{1}\right)\right]\left[\gamma\left(t_{1}\right)\right],
\end{aligned}
$$


where

$$
\begin{aligned}
\mathbf{X}\left(t_{k+1}\right) \mid \gamma, \mathbf{X}\left(t_{1: k}\right) & \sim N_{2}\left(\mathbf{X}\left(t_{k}\right)+\gamma\left(t_{k}\right) \frac{\mathbf{X}\left(t_{k}\right)-\mathbf{X}\left(t_{k-1}\right)}{t_{k}-t_{k-1}}\left(t_{k+1}-t_{k}\right),\right. \\
\left.\Sigma_{\mathbf{X}}(\boldsymbol{\theta}) \sqrt{t_{k+1}-t_{k}}\right) & \\
\gamma\left(t_{k+1}\right) \mid \gamma\left(t_{1: k}\right) & \sim N_{1}\left(\gamma\left(t_{k}\right), \sigma_{\gamma}(\boldsymbol{\theta}) \sqrt{t_{k+1}-t_{k}}\right) .
\end{aligned}
$$

The distributions in $(7.12)$ are obtained directly from the model formulation in (7.11).

To give an example of latent state estimation using this model, we simulated data using the movement process in $(7.11)$ but where the latent behavioural states are simulated using a sine-wave rather than a random walk, following Auger-Méthé et al. (2017); Breed et al. (2012). We generated 1 track consisting of 300 observations taken at equidistant time intervals, which were assumed to be the true locations using the covariance matrix $\Sigma_{X}(\boldsymbol{\theta})=I_{2}$. This track is shown in the left panel of Figure 7.5 with each location divided into arbitrary groups of directed travel $(\gamma(t) \geq 0.7)$ in pink and foraging $(\gamma(t)<0.3)$ in blue. The right panel of Figure 7.5 plots the true behavioral states in red, with the estimates obtained via the mode of $[\gamma \mid \mathbf{X}]$ shown in black. The shaded region is the $95 \%$ confidence bound on these estimates calculated as \pm 2 standard errors. This confidence bound contains the true values at all times. 


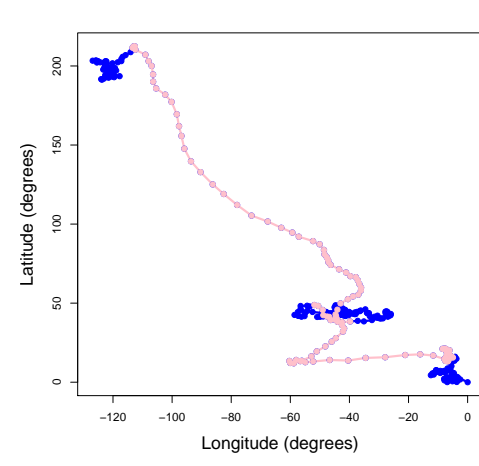

(a) Simulated track

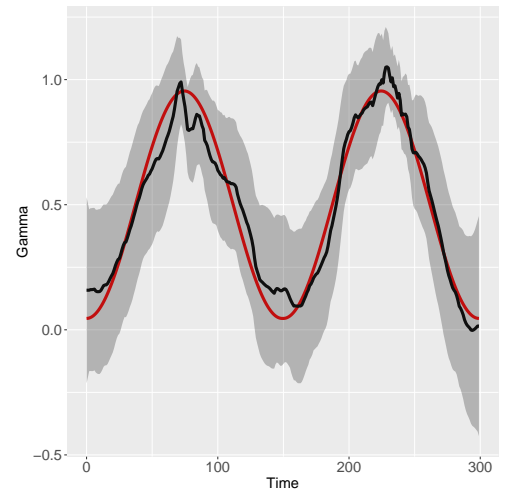

(b) $\gamma(t)$ 's

Figure 7.5: Left Panel: Simulated data consisting of 300 sampling location observations taken at equidistant intervals, where pink lines show movement in the travelling state $(\gamma(t) \geq 0.7)$ and blue lines in the foraging state $(\gamma(t)<0.7)$ following Auger-Méthé et al. (2017). Right Panel: True latent behavioural state $\gamma(t)$ used to generate the track as shown by the red line. The black line and shaded region are the $\hat{\gamma}$ estimates and $95 \%$ confidence intervals obtained using TMB. 


\section{Chapter 8}

\section{Preferential Sampling of Ocean Temperatures}

In this chapter we propose a flexible framework for inference on preferentially sampled fields, where the process that generates the sampling locations is stochastic and is moving over time through a 2-dimensional space. This space may be some transformed version of the coordinate system, an idea which we introduce in Section 8.5. Our simulation studies confirm that predictions obtained from the preferential sampling model are more reliable when this phenomenon is present and comparable to the standard ones when it is not. The application in this thesis is the preferential sampling of ocean temperatures by tagged marine mammals, but these methods can be easily adapted to other settings where samples are taken from moving objects.

We first review the use of marine mammals and ocean temperature samplers (bio-loggers) in Section 8.1 and introduce preferential sampling from bio-loggers in Section 8.2. The data which is to be modelled along with the modelling assumptions we will make are introduced in Sections 8.2.1 and 8.2 .2 respectively. This is followed by our proposition of a novel movement model that captures possible preferential velocities in Section 8.3. Finally we report the results of our simulation studies in Section 8.4 and discuss the results of a real data set analysis in Section 8.5.

\subsection{Marine Bio-logging}

The rise of global ocean temperatures over recent years was first quantified by Levitus et al. (2000) using 50 years of historical data between 1948-1998, who concluded that during this period global ocean temperature change was most likely a combination of human activity such as pollution and natural variability. Jacobs $(2006)$ supports this view, highlighting the change in Southern Indian ocean temperatures, which appear to be rapidly warming in areas up to 3000 meters below sea level. However, one issue with polar areas such as the Antarctic and Southern Indian ocean is the lack of data 
in comparison with other, easier to sample ocean regions. Sea ice is a major obstacle for research vessels and Argo floats which are commonly used to profile ocean temperatures, hence data collection is biased towards summer months and even then still sparsely available. Furthermore, the Argo float system is designed for broad-scale sampling of the oceans, where floats are separated by hundreds of kilometers and therefore does not capture fine scale change such as eddies and boundary currents (Boehme et al., 2009; Roemmich et al., 2004).

Fedak (2004) highlighted the potential of marine mammals to perform oceanographic sampling, through the deployment of tags originally used to provide information on their immediate environment and habitats. As we covered in Section 7.1, marine mammal mounted tags were originally used to track animal movement but advances in technology meant that by the 21st Century these tags could measure environmental variables such as salinity and temperature. This CTD (Conductivity-Temperature-Depth) sensor data was first collected using marine mammals by Lydersen et al. (2002), who used white whales to collect data in an an Arctic fjord in Svalbard. By 2012 there had been a huge increase in the quantity of CTD data that had been collected, with over 1.4 million T/D (temperature/depth) and CTD profiles available in the World Ocean Database (WOD, https: //www.nodc.noaa.gov/)(Boyer et al., 2013 Fedak, 2013). In this time multiple projects such as SEaOS (Southern Elephant Seals as Oceanographic Samplers http://biology.st-and.ac.uk/seaos/index.html) and subsequently MEOP (Marine Mammals Exploring the Oceans Pole to Pole http://www.meop.info/) had been set up to further the understanding of polar ocean regions using marine mammal collection platforms. By 2014 the MEOP-CTD database had 200,000 profiles primarily collected from elephant seals with approximately 75,000 of these profiles obtained in the Southern Indian ocean (Roquet et al., 2014b, 2013). More than $45 \%$ of these profiles were obtained south of $60^{\circ} \mathrm{S}$ which is an area of sampling sparsity, hence providing scientists with an abundance of new oceanographic information.

\subsection{Preferential Sampling of Ocean Temperatures}

Although CTD data collected by marine mammals is successfully helping to fill out gaps in our knowledge of ocean temperatures, these platforms are still unable to cover vast areas of water. Therefore, geostatistical methods of interpolation such as kriging can be used to fill in these gaps, and provide 
predictions at unsampled locations. Kriging can be viewed as a weighted balance between the estimate of the local mean field and the observed data offering a statistical alternative to deterministic model interpolation. An overview of kriging can be found in Section 2.3.2. Historically, deterministic (non-statistical) models which represent the underlying thermal physics mathematically have provided a useful approach for management purposes in understanding cause and effect relationships, but these methods rely on a large number of input parameters which are regularly unavailable in sparsely sampled areas (Benyahya et al., 2007; Caissie et al., 2001). Examples of kriging use in water temperature prediction include Daigle et al. (2010) who used the method to predict water temperature in the Okanagan Watershed, whilst Ozturk and Kilic (2016) used kriging for interpolation of bottom water temperatures obtained from Conductivity-Temperature-Depth tag (CTD) transects on the Norwegian-Svalbard continental margin. This work utilised the $R$ package INTAMAP (Pebesma et al., 2011) which offers a variety of prediction techniques for spatial data.

One area which has not been considered however, is the impact that preferential sampling may cause in ocean temperature profile prediction. For example, one of the most common tagged mammals in ocean profiling are elephant seals. These mammals are likely to adjust their foraging due to the warming of ocean temperatures (McIntyre et al., 2011), because their prey tends to prefer cooler and deeper waters. It also appears that there are less successful forages when diving in warmer water (Guinet et al., 2014). The case when the process that determines the data locations and the spatial field of interest may depend on each other, as can be the case with data collected from animal-mounted tags, is preferential sampling as described in Chapter 3 .

To investigate the potential impact that preferential sampling of ocean temperatures might have on inference, we first define a model for the CTD data obtained from marine mammals. In this chapter we consider sea surface temperature (SST) data only. Hence we assume the SST measurements to be water temperature values obtained from the tag taken at the closest time point to observed locations of the mammal, which can only be obtained at the surface. Therefore data consists of SST readings

$$
Y_{k}=S\left(\mathbf{X}\left(t_{k}\right)\right)+\epsilon_{k}
$$

where $\mathbf{X}\left(t_{k}\right)$ is the sampling location at time $t_{k}$ and $S\left(\mathbf{X}\left(t_{k}\right)\right)$ is the true SST at that location with $\epsilon_{k}$ some measurement error. For now we will assume that $S$ is a stationary field with constant mean and a Matérn covari- 
ance from (2.4), although we discuss relaxing the stationarity assumption in Section 9.2 .

To estimate the parameters of interest in the model, we again utilise a Laplace approximation to the likelihood similar to that described in Section 4.4, where the dependence between the sampling locations and the field of interest (SST) results in a likelihood that is computationally challenging to optimize. We follow previous chapters in using the flexible TMB package in $R$ to deal with the computational complexity of the preferential sampling analysis. Therefore we will need to define the joint negative log-likelihood from (4.21) in the case of preferentially sampled SST data $f(\mathbf{S}, \boldsymbol{\theta})=-\log ([\mathbf{Y} \mid \mathbf{S}, \mathbf{X}][\mathbf{X} \mid \mathbf{S}][\mathbf{S}])$. Since we are retaining the same measurement equation in (8.1) as previously in (2.1) and assuming $S$ is stationary with constant mean and Matérn covariance, then $[\mathbf{Y} \mid \mathbf{S}, \mathbf{X}][\mathbf{S}]$ have the same structure as in the stationary preferential sampling case from Chapters 4,5. However, the distribution of sampling locations $[\mathbf{X} \mid \mathbf{S}]$ will certainly change, which we discuss in Section 8.3 .

It is interesting to note that the effect of preferential sampling in these "dynamic" spatial models (where sampling locations move through the domain) can be expected to be different from what happens when sampling locations are stationary. In the latter, when there is no preferential sampling, sampling locations are usually assumed to have been uniformly sampled over the spatial domain. Thus, when preferential sampling is present it often results in distinct and potentially informative patterns in the stationary sampling locations (Diggle et al., 2010; Dinsdale and Salibian-Barrera, 2018). In contrast, even without preferential sampling, the locations visited by the animals in our study would typically not be expected to be uniformly distributed over the area of interest due to the dynamic nature of their movement. As a result, the information about SST contained in the locations the animals visited (or in the regions without observations) may be less apparent for these dynamical models. Nevertheless, we show that not taking into account preferential sampling may still affect negatively the quality and reliability of the estimated parameters and predictions.

\subsubsection{Data Description}

In this thesis we will be analysing data that were collected and made freely available as part of the MEOP database and we will in particular utilise the South Indian ocean data subset described by Roquet et al. (2014b). We use the location, temperature and depth measurements of the CTD sensor data made available by Roquet et al. (2014a). Data is collected during the 
deepest dive within a 6 hour time interval (Roquet et al., 2011). Once the animal reaches the surface of this dive, the location is determined using the ARGOS satellite system and are typically accurate within \pm 5 kilometers, whilst the temperatures are accurate within $\pm 0.03^{\circ} \mathrm{C}$ (Roquet et al., 2014b). In total this data set consists of 1,169,979 observations taken between 2 nd February 2008 and 10th March 2013 at depths ranging from 6 to 2000 meters. For analysis later in this chapter, we will be considering what we denote as sea surface temperature (SST) readings from these tags. In reality these readings are taken at a depth of 6 meters. We will analyse data at greater depths in Section 9.1. In total the SST subset consists of 66,519 observations, which we plot in Figure 8.1 .

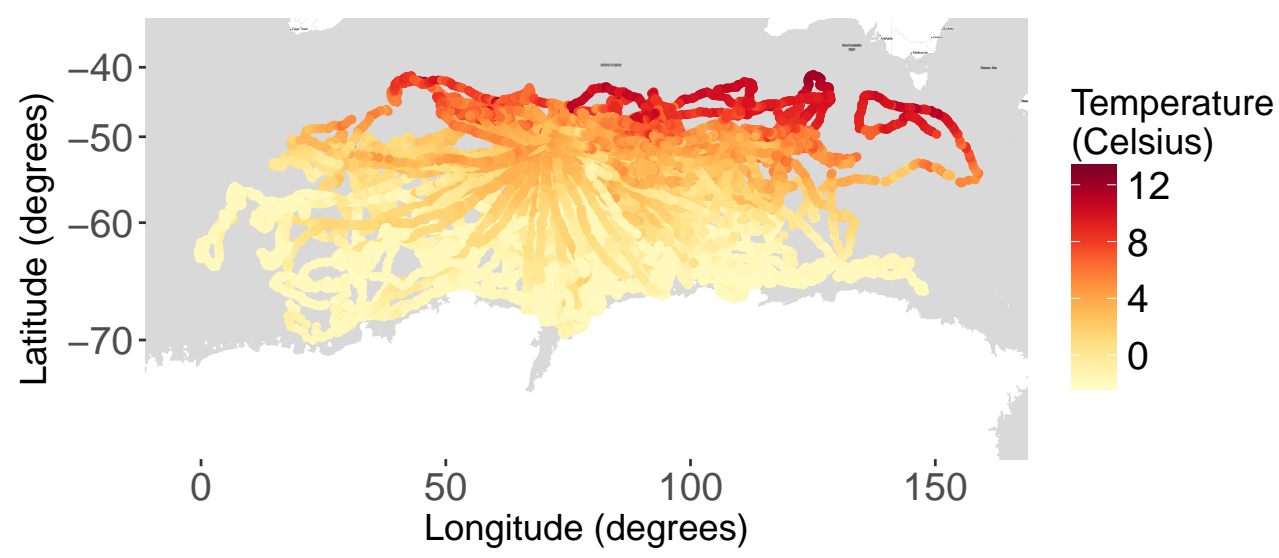

Figure 8.1: Plot of all 66,519 sea surface temperature (SST) observations from Southern elephant seal platforms taken between 2nd February 2008 and 10th March 2013. SST's were approximated as the temperature at the maximum depth of 6 meters. Map tiles by Stamen Design, under CC BY 3.0. Data by OpenStreetMap, under ODbL.

For predictive assessment of the ocean temperatures obtained from marine mammal tags we will compare our model predictions with monthly average temperature fields obtained via the Simple Ocean Data Assimilation ocean/sea ice reanalysis (SODA) (Carton and Giese, 2008) which uses all temperature and salinity profiles from the World Ocean Database, specif- 
ically the SODA version 3 (SODA3) reanalysis (Carton et al., 2018). This data is available for depths between 5 and 5000 meters below the surface at a spatial resolution of 0.5 degrees latitude and longitude. Data is available from January 1980 to present.

\subsubsection{Assumptions}

In this thesis we will be assuming that although the samples are taken at various time points, that the temperatures $S(\cdot)$ depend only on location and not time. Therefore we can view the continuous SST field $S$ as fixed, with samples being obtained at various locations $\mathbf{X}\left(t_{k}\right)$. Further research in this area relaxing this assumption would be valuable and we discuss this further in the future work Section 10.1.3. Enabling $S$ to vary over time, as it would in real life, would allow for analysis of data over longer periods of time with the model adapting to SST over various seasons and years. In this case one would consider the data to be of the form $Y_{k}=S\left(\mathbf{X}\left(t_{k}\right), t_{k}\right)+\epsilon_{k}$ so that $S(\cdot)$ is a function of both location and time.

Another are of future development is to consider data at any depth. However, since location information below the surface is less accurate we do not consider such data in this chapter. Theoretically nothing would change in comparison to the SST data we consider throughout this section, however the location accuracy underwater is reduced. In this case location estimates could inferred based on accelerometer data using methods such as the dead reckoning algorithm (Liu et al., 2015; Wilson and Wilson, 1988) and ocean temperature at specific depths analysed to build a water temperature profile. We conduct initial analysis of ocean data at depths in Section 9.1.

\subsection{A Preferential Model for Marine Mammal Movement}

To account for preferential sampling of ocean temperatures, we need to define a model for the location of marine mammals that takes into account possible relationships between movement velocity and ocean temperature. In Section 7.2 we covered some typical marine mammal movement models using SDEs such as the OU model and also the DCRW and CTCRW models which incorporate velocity. If we consider the 2-dimensional continuous movement modelled via an SDE as defined in (2.15), then under preferential sampling one would need to define an SDE in which the expected movement 


\subsection{A Preferential Model for Marine Mammal Movement}

depends on the ocean temperature $S$. For example, a model of the form

$$
\mathrm{d} \mathbf{X}(t)=\boldsymbol{\mu}(\mathbf{X}(t), S, \boldsymbol{\theta}) \mathrm{d} t+\boldsymbol{\Sigma}(\boldsymbol{\theta}) \mathrm{d} \boldsymbol{B}(t),
$$

where $\boldsymbol{\theta}$ is a vector of all model parameters. Here the drift function, $\boldsymbol{\mu}(\cdot)$, which describes expected movement direction and velocity is a function of $S$.

In practice we will be using discretised models as functions of the observed locations $\mathbf{X}=\left(\mathbf{X}\left(t_{1}\right), \ldots, \mathbf{X}\left(t_{n}\right)\right)$, in which movement may also depend on previous locations. In these cases, the non-preferential models from Section 7.2 write the location at time $t_{k+1}$ as

$$
\mathbf{X}\left(t_{k+1}\right)=g_{\mathrm{NP}}\left(\mathbf{X}\left(t_{1: k}\right), \boldsymbol{\theta}\right)+\epsilon\left(\mathbf{t}_{1: k}, \boldsymbol{\theta}\right)
$$

where $\mathbf{X}\left(t_{1: k}\right)=\left(\mathbf{X}\left(t_{1}\right), \ldots, \mathbf{X}\left(t_{k}\right)\right)$ and $\boldsymbol{\theta}$ is the vector of all model parameters. The function $g_{\mathrm{NP}}(\cdot)$ is some deterministic movement function where NP stands for non-preferential and $\epsilon(\cdot)$ is an error term.

Using the representation in (8.3), under preferential sampling we need to define a movement model of the form

$$
\mathbf{X}\left(t_{k+1}\right)=g_{\mathrm{P}}\left(\mathbf{X}\left(t_{1: k}\right), S, \boldsymbol{\theta}\right)+\epsilon\left(\mathbf{t}_{1: k}, \boldsymbol{\theta}\right)
$$

where the function $g_{\mathrm{P}}$ is now a function of $S$, therefore enabling the movement model to depend on the temperature field.

\subsubsection{A Preferential Correlated Random Walk Model}

We consider a model similar to the first-difference correlated random walk (DCRW) model (Jonsen et al., 2005) as defined in (7.6). In our case we want to include non-regular time intervals and also some type of behavioural state to account for different movement patterns such as foraging and directed movement. This leads us to a movement model similar to that in (7.11) which we will term the "preferential correlated random walk" (PCRW) model.

We assume that the sampling locations $\mathbf{X}\left(t_{1}\right), \ldots, \mathbf{X}\left(t_{n}\right)$ follow

$$
\mathbf{X}\left(t_{k+1}\right)=\mathbf{X}\left(t_{k}\right)+\boldsymbol{\mu}\left(\mathbf{X}\left(t_{1: k}\right), S\right)\left(t_{k+1}-t_{k}\right)+\boldsymbol{\Sigma}_{x} \boldsymbol{A}_{k} \sqrt{t_{k+1}-t_{k}},
$$

where $\mathbf{A}_{k}$ denotes a standard bivariate normal random vector, $\boldsymbol{\Sigma}_{x}$ is a $2 \times 2$ matrix that corresponds to the variance of the diffusion terms, and $t_{k}$ are the observation times. The drift function and behavioural states at the measured locations and times satisfy:

$$
\boldsymbol{\mu}\left(\mathbf{X}\left(t_{1: k}\right), S\right)=f\left(\beta_{t_{k}}\right) \boldsymbol{\phi}\left(\mathbf{X}\left(t_{k}\right), S\right)+\left(1-f\left(\beta_{t_{k}}\right)\right) \mathbf{v}\left(\mathbf{X}\left(t_{1: k}\right)\right)
$$




\subsection{A Preferential Model for Marine Mammal Movement}

where $\beta_{t_{k}} \in \mathbb{R}$ for all $t_{k}, \mathbf{v}: \mathbb{R}^{2} \rightarrow \mathbb{R}^{2}$ represents the "velocity" of the animal, and $\phi: \mathbb{R}^{2} \times \mathbb{R}^{2} \rightarrow \mathbb{R}^{2}$ can be thought of as the foraging movement function that depends on the location and the latent temperature field $S$, which models the possible preference of the animals for different water temperatures. The behavioural state function $f: \mathbb{R} \rightarrow[0,1]$ depends on $\beta_{t_{k}}$ and controls the auto-correlation of the movement at each time point. This ensures that when $f\left(\beta_{t_{k}}\right) \approx 1$ then $\phi\left(\mathbf{X}\left(t_{k}\right), S\right)$ becomes the expected drift direction, whilst when $f\left(\beta_{t_{k}}\right) \approx 0$ movement tends in the direction of the current velocity $\mathbf{v}\left(\mathbf{X}\left(t_{1: k}\right)\right)$.

It is important to note that the velocity function may depend on more than just the previous sampling location. Consequently, like the DCRW, our PCRW model is not Markovian. Although one may consider including a latent velocity state $(\mathbf{v})$ similar to the continuous time correlated random walk model (CTCRW) of Johnson et al. (2008), if this velocity was to depend on the locations $\mathbf{X}$ and also $S$, such a model becomes drastically more complicated. We discuss this in more detail in Section 8.3.2. Therefore we consider a non-latent velocity state approximation, taken to be

$$
\mathbf{v}\left(\mathbf{X}\left(t_{1: k}\right)\right)=\frac{\mathbf{X}\left(t_{k}\right)-\mathbf{X}\left(t_{k-1}\right)}{t_{k}-t_{k-1}}
$$

Next, we specify a behavioural function

$$
f\left(\beta_{t_{k}}\right)=\frac{\exp \left(\beta_{t_{k}}\right)}{1+\exp \left(\beta_{t_{k}}\right)},
$$

so that as $\beta_{t_{k}}$ increases, so does the influence of $\phi$, whereas when $\beta_{t_{k}}$ decreases the current velocity $\mathbf{v}\left(\mathbf{X}\left(t_{k}\right)\right)$ becomes more of a factor in the movement. Therefore, our PCRW model can be written

$$
\begin{aligned}
\boldsymbol{\mu}\left(\mathbf{X}\left(t_{k}\right), S\right) & =\frac{\exp \left(\beta_{t_{k}}\right)}{1+\exp \left(\beta_{t_{k}}\right)} \boldsymbol{\phi}\left(\mathbf{X}\left(t_{k}\right), S\right)+\frac{1}{1+\exp \left(\beta_{t_{k}}\right)} \mathbf{v}\left(\mathbf{X}\left(t_{1: k}\right)\right), \\
\beta_{t_{k+1}} & =\beta_{t_{k}}+\sigma_{\beta} B_{k} \sqrt{t_{k+1}-t_{k}},
\end{aligned}
$$

where $B_{k}$ are univariate standard normal random variables and $\sigma_{\beta}>0$ determines the evolution of the random states $\beta_{t_{k}}$. Note that the inclusion of the random $\beta$ states means we need to re-specify the preferential likelihood for our Laplace approximation, which we show in Section 8.3.2.

Depending on the application of the PCRW model, a variety of forms for the foraging function $\phi$ may be used. In our case, to model the possible tendency of an animal to move towards particular water temperatures we propose the following:

$$
\boldsymbol{\phi}(\mathbf{X}, S)=-\alpha S(\mathbf{X}) \nabla S(\mathbf{X}),
$$


where $\alpha \in \mathbb{R}, S(\mathbf{X})$ is the value of the random field at location $\mathbf{X}$ and $\nabla S(\mathbf{X})$ is the gradient of $S$ at $\mathbf{X}$. Although the parameter $\alpha$ above may appear to be unidentifiable, this is in fact not the case when you consider the full likelihood function which also includes the density functions $[\mathbf{Y} \mid S, \mathbf{X}]$ and $[S]$.

The form of $\phi$ in (8.9) defines the expected drift as descending along the gradient of the SST field, with a velocity that depends both on the temperature at the present location and a scalar $\alpha$. This is similar to the varying motility surface used by Russell et al. (2018) to allow the magnitude of the velocity vector to depend on the location. More specifically, we can view the latent field $S$ as a scaled potential surface in which the gradient of $S$ directs the expected movement with a velocity that also depends on the value of $S$ at that location. Potential surfaces have previously been used to model the movement of animals including monk seals (Brillinger et al., 2008), elk (Brillinger et al., 2002; Preisler et al., 2013), and ants (Russell et al., 2018).

It should be noted that appropriate forms of $\phi$ for particular species may require specialist input and we do not claim that the one shown in (8.9) is necessarily the best model for seals. The preferential sampling effect may vary over species, locations and possibly even individuals. However, the form in (8.9) may identify preferential movement and we use it as an example for integrating a foraging function into the preferential sampling framework. Furthermore, in application we may wish to adjust $(8.9)$ to

$$
\phi(\mathbf{X}, S)=-\alpha S^{*}(\mathbf{X}) \nabla S(\mathbf{X})
$$

where $S^{*}=S+c$ for some constant $c \in \mathbb{R}$ specified by the user. We would do this to ensure that $S^{*}$ is the same sign across the domain, to prevent the switching of movement patterns when $S$ goes from negative to positive or vice versa.

\subsubsection{Evaluating the Preferential Correlated Random Walk Model}

In Section 4.4 we outlined how to derive the preferential model using a Laplace approximation to the likelihood function. In previous cases this factorisation was of the form $[\mathbf{X}, \mathbf{Y}]=\int[\mathbf{X}, \mathbf{Y}, \mathbf{S}] \mathrm{d} \mathbf{S}$, however in the case of the PCRW model defined in Section 8.3.1 we have a second latent vector in the form of behavioural states $\boldsymbol{\beta}=\left(\beta\left(t_{1}\right), \ldots, \beta\left(t_{n}\right)\right)$. Therefore we need to re-specify the full likelihood for our Laplace approximation routine. This 
can be done in a similar way to before as

$$
\begin{aligned}
{[\mathbf{X}, \mathbf{Y}] } & =\iint[\mathbf{X}, \mathbf{Y}, \mathbf{S}, \boldsymbol{\beta}] \mathrm{d} \mathbf{S} \mathrm{d} \boldsymbol{\beta}, \\
& =\iint[\mathbf{Y} \mid \mathbf{X}, \mathbf{S}, \boldsymbol{\beta}][\mathbf{X}, \mathbf{S}, \boldsymbol{\beta}] \mathrm{d} \mathbf{S} \mathrm{d} \boldsymbol{\beta}, \\
& =\iint[\mathbf{Y} \mid \mathbf{X}, \mathbf{S}, \boldsymbol{\beta}][\mathbf{X} \mid \mathbf{S}, \boldsymbol{\beta}][\mathbf{S}, \boldsymbol{\beta}] \mathrm{d} \mathbf{S} \mathrm{d} \boldsymbol{\beta}, \\
& =\iint[\mathbf{Y} \mid \mathbf{X}, \mathbf{S}, \boldsymbol{\beta}][\mathbf{X} \mid \mathbf{S}, \boldsymbol{\beta}][\boldsymbol{\beta} \mid \mathbf{S}][\mathbf{S}] \mathrm{d} \mathbf{S} \mathrm{d} \boldsymbol{\beta} .
\end{aligned}
$$

Notice that in the PCRW model we have $[\boldsymbol{\beta} \mid \mathbf{S}]=[\boldsymbol{\beta}]$, so therefore in the Laplace approximation implemented using TMB we will need to redefine the negative joint log-likelihood as

$$
f(\mathbf{S}, \boldsymbol{\theta})=-\log ([\mathbf{X}, \mathbf{Y}, \mathbf{S}, \boldsymbol{\beta}])=-\log ([\mathbf{Y} \mid \mathbf{X}, \mathbf{S}, \boldsymbol{\beta}][\mathbf{X} \mid \mathbf{S}, \boldsymbol{\beta}][\boldsymbol{\beta}][\mathbf{S}]),
$$

where we use a finite-differences approximation to the gradient of the field $S$ to construct a likelihood function that can be maximized numerically.

Alternatively one may consider including a latent velocity state that also depends on $S$, for example a preferential equivalent to the CTCRW from (7.8)-(7.9), but such a model becomes drastically more complicated. To see this, we observe the factorisation of the full likelihood for the latent velocity model in which the velocity depends on the underlying field $S$. In this case we specify a full likelihood as

$$
\begin{aligned}
{[\mathbf{X}, \mathbf{Y}] } & =\iint[\mathbf{X}, \mathbf{Y}, \mathbf{S}, \mathbf{v}] \mathrm{d} \mathbf{S} \mathrm{d} \mathbf{v}, \\
& =\iint[\mathbf{Y} \mid \mathbf{X}, \mathbf{S}, \mathbf{v}][\mathbf{X}, \mathbf{S}, \mathbf{v}] \mathrm{d} \mathbf{S} \mathrm{d} \mathbf{v}, \\
& =\iint[\mathbf{Y} \mid \mathbf{X}, \mathbf{S}, \mathbf{v}][\mathbf{X} \mid \mathbf{S}, \mathbf{v}][\mathbf{S}, \mathbf{v}] \mathrm{d} \mathbf{S} \mathrm{d} \mathbf{v} \\
& =\iint[\mathbf{Y} \mid \mathbf{X}, \mathbf{S}, \mathbf{v}][\mathbf{X} \mid \mathbf{S}, \mathbf{v}][\mathbf{v} \mid \mathbf{S}][\mathbf{S}] \mathrm{d} \mathbf{S} \mathrm{d} \mathbf{v},
\end{aligned}
$$

where $\mathbf{v}$ denotes the vector of velocities and to simplify notation we do not include a behavioural state. Notice that this factorisation includes the term $[\mathbf{v} \mid \mathbf{S}]$. If the velocity was to only depend on the latent field $\mathbf{S}$ and not on the location $\mathbf{X}$, then we could evaluate the likelihood. However, this makes little biological sense in practice. Preferential movement induced by a latent velocity would require this velocity to depend on the field $\mathbf{S}$ at the 
current location $\mathbf{X}$ of the animal. In other words the velocity would be dependent not just on the latent field but the current location within this field. However, this means that we would only have a tractable form for $[\mathbf{v} \mid \mathbf{S}, \mathbf{X}]$ and would therefore need to evaluate

$$
\begin{aligned}
{[\mathbf{v} \mid \mathbf{S}] } & =\int[\mathbf{X}, \mathbf{v} \mid \mathbf{S}] \mathrm{d} \mathbf{X}, \\
& =\int[\mathbf{v} \mid \mathbf{S}, \mathbf{X}][\mathbf{X} \mid \mathbf{S}] \mathrm{d} \mathbf{X},
\end{aligned}
$$

which is difficult to compute due to the complexity of $[\mathbf{X} \mid \mathbf{S}]$ which is itself

$$
[\mathbf{X} \mid \mathbf{S}]=\int[\mathbf{X} \mid \mathbf{S}, \mathbf{v}][\mathbf{v} \mid \mathbf{S}] \mathrm{d} \mathbf{v} .
$$

The factorisation of the form in $(8.12)$ appears intractable and therefore a preferential CTCRW model in which velocities depend on location and the latent field becomes difficult to implement. This is one of the reasons we use a non-latent approximation to the velocity in the drift function of the PCRW model in (8.8), the other is to reduce the number of latent states in the PCRW model which would total three $(\mathbf{v}, \boldsymbol{\beta}, \mathbf{S})$ in this case.

\subsubsection{Advantage in Comparison to Point Process Model}

In this section we give a brief discussion on the approach of using animal movement models rather than a point process model similar to those used in Sections 4 6, since the approaches discussed in this chapter add a level of computational complexity to calculating $[\mathbf{X} \mid S]$ not found when using the intensity from (3.4).

The main concern with using point process models is the lack of dependency between locations, conditional on the latent field $S$. We are assuming in our point process examples than for a given field $S$, the sampling locations are independent of each other. This is not the case in our movement models, where each sampling location depends on the previous locations. We can use this to incorporate a relationship, not just between sampling locations, but the movement which drives these locations. For example, the PCRW model we introduced in Section 8.3.1 enables movement to depend on gradients of the latent field $S$. In other words, the change in $S$ influences sampling locations, rather than just the value of $S$. This is something we do not believe is possible using point process models. Hence, even if we used a more complex stationary sampling model to try and incorporate dependence 


\subsection{Simulation Experiments}

between locations, the idea of relative change in $S$ influencing our sampling locations would be difficult to implement.

It is for this reason that we utilise animal movement literature to develop our own movement model rather than try to adapt a point process. We believe that in the case of moving processes that generate sampling locations, it is key to understand what is driving the movement, rather than just the sampling locations.

\subsection{Simulation Experiments}

In this section we discuss the results of a simulation study conducted to illustrate the effect of preferential sampling on the analysis of spatial data where sampling locations are moving through space, such as is the case in our ocean temperature data. The goal is to show to what extent incorporating preferential sampling in the model may improve the resulting SST predictions and parameter estimates. Since the locations visited by the animals in our study would not typically be uniformly distributed over the area of interest (even without the presence of preferential sampling), the information about ocean temperatures included in their locations may not be easily inferred visually. However, our experiments described below show that important gains in prediction accuracy and parameter estimation can be obtained by using a model that incorporates the possibility of preferential sampling. Furthermore, we also show that when preferential sampling is not present, the results obtained using a model that incorporates preferential sampling are very similar to those obtained using the usual geostatistical model that conditions on the locations.

\subsubsection{Simulation 1}

In this simulation we generated 50 data sets, each of them consisting of up to 300 observations across 3 animal tracks simulated following the PCRW movement model described in Section 8.3.1, specifically (8.8)-(8.9). The tracks were initiated at a location chosen uniformly at random over the 2-dimensional domain $[-150,150] \times[-150,150]$, and the first 20 positions of each animal were considered a burn-in period and discarded. For each data set we simulated a random field $S$ over the domain and discretised it on a $51 \times 51$ grid. We used a Matérn covariance function as in (2.4) with smoothness parameter $\kappa=2$, scale $\phi=15$, marginal variance $\sigma^{2}=3$ and a constant mean $\mu=5$. For the movement model we set $\boldsymbol{\Sigma}_{x}=2.5 \mathbf{I}_{2}$, where $\mathbf{I}_{2}$ denotes the $2 \times 2$ identity matrix, $\sigma_{\beta}=0.1, \alpha=80$ and initiated the 


\subsection{Simulation Experiments}

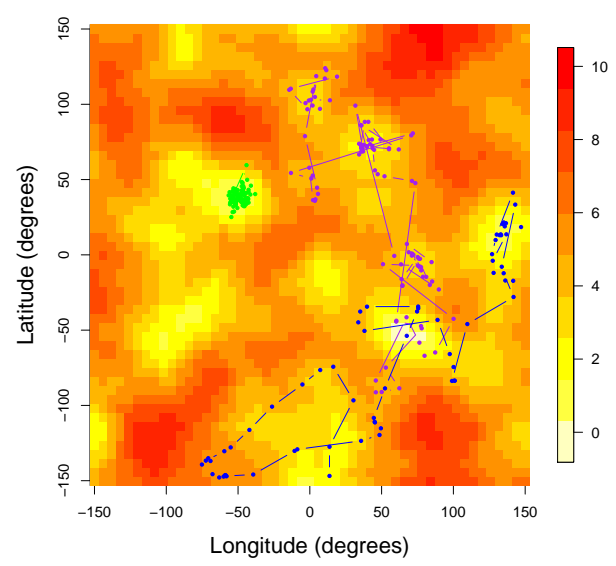

Figure 8.2: Example of a simulated data set of 3 tracks generated using the field and movement parameters defined in simulation 1, resulting in a moderate preferential sampling effect.

behavioural states at $\beta_{1}=0$. As is commonly done in the literature, we assumed that the smoothness parameter $\kappa$ was known. Finally, the time between consecutive observations followed an exponential distribution with rate parameter $\lambda=1$. With these set of movement parameters we expect the tracks to oversample cooler regions. Figure 8.2 shows a randomly chosen data set generated as described above where the preferential sampling effect is apparent.

In the rest of this section we first compare parameter estimates obtained using the standard likelihood with those using the preferential model implemented using TMB. We then study the SST predictions resulting from ordinary kriging using the parameter estimates obtained with the standard likelihood and those obtained from the mode of the estimated predictive distribution acquired from the preferential model (4.14).

\section{Parameter Estimates}

Figure 8.3 shows the boxplots of the 50 parameters estimates for the spatial process using each of the two likelihoods (standard and accounting for preferential sampling). The black horizontal lines represent the true values. As expected, when the model did not account for preferential movement, the prevalence of lower temperatures in the sample introduced a negative 


\subsection{Simulation Experiments}

bias to the estimates for the mean parameter $(\mu)$. A similar pattern was also observed for the scale $(\phi)$ and marginal variance $\left(\sigma^{2}\right)$. The bias in the variance estimates was likely due to the tracks avoiding higher temperature regions and recording temperatures with a reduced range than they would otherwise, a phenomena which was also present in the preferential simulation using point processes in Section 4.5. This movement pattern may also explain the negative bias in the scale estimates. In contrast, the estimates obtained from the PCRW model using TMB showed correction for the bias in these parameter estimates. This was particularly noticeable for the $\mu, \phi$ and $\sigma^{2}$ estimates. However, it should be noted that there appears to be overcorrection for $\phi$ and to a lesser extent $\sigma^{2}$. The estimated nugget variances $\left(\tau^{2}\right)$ appear to be very similar for both methods. Figure 8.4 shows that the movement parameters were also estimated accurately, although there does appear to be a slight under estimation of $\alpha$. The positive $\hat{\alpha}$ 's account for the tendency of the sampler to avoid warmer warmers, which explains the correction to the mean parameter estimates observed in Figure 8.3 .

We also generated 50 data sets with non-preferentially sampled data (i.e. data following the PCRW model in Section 8.3 but with $\alpha=0$ in $(8.9)$ ). For each of these 50 data sets we estimated the parameters of the spatial process using the PCRW likelihood and the standard one (that conditions on the observed locations). The results in Figure 8.5 show that, as expected, there was no practical difference between the parameter estimates obtained using either likelihood.

\section{Prediction}

We now turn our attention to the predictions for the underlying spatial field of interest $S$. We compare the predictions for the spatial field obtained using a standard spatial model with those resulting from the preferential sampling model. The first set of predictions were computed via kriging with parameter estimates obtained from the standard model that conditions on the observed locations, while the preferential sampling ones correspond to the estimated mode of $[\mathbf{S} \mid \mathbf{X}, \mathbf{Y}]$ provided by TMB (see $(4.14)$ ).

Predictions were computed on a $26 \times 26$ lattice for each of the $M=50$ data sets and we used two different measures of their quality. The rootmean-square-prediction error (RMSPE) over the discrete domain is given by

$$
\operatorname{RMSPE}_{i}=\frac{1}{M} \sum_{j=1}^{M} \sqrt{\left(\hat{\mathbf{S}}_{j, i}-\mathbf{S}_{j, i}\right)^{2}}
$$




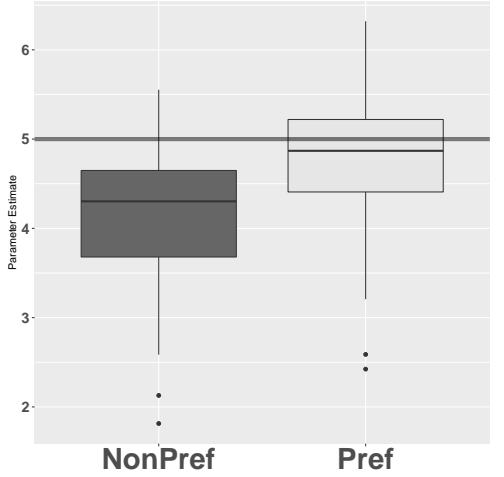

(a) Mean $(\mu)$

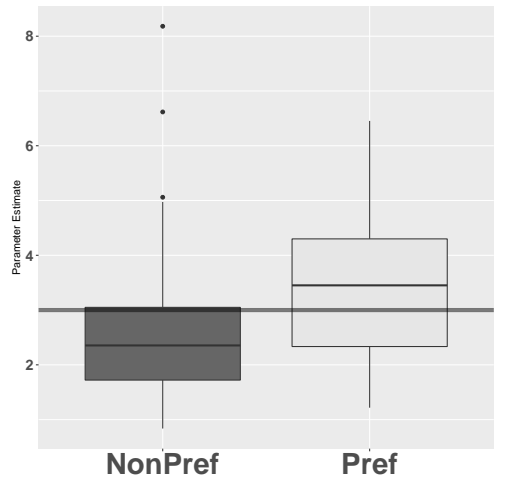

(c) Variance $\left(\sigma^{2}\right)$

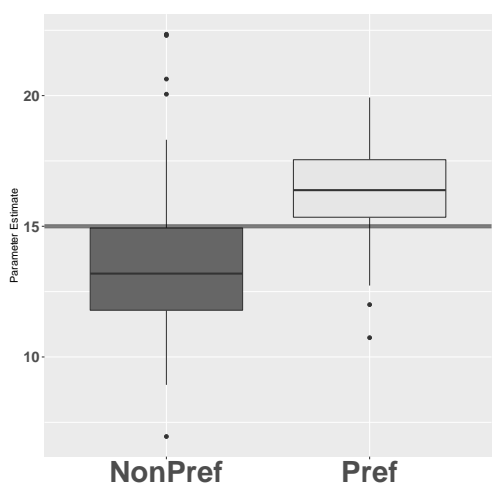

(b) Scale $(\phi)$

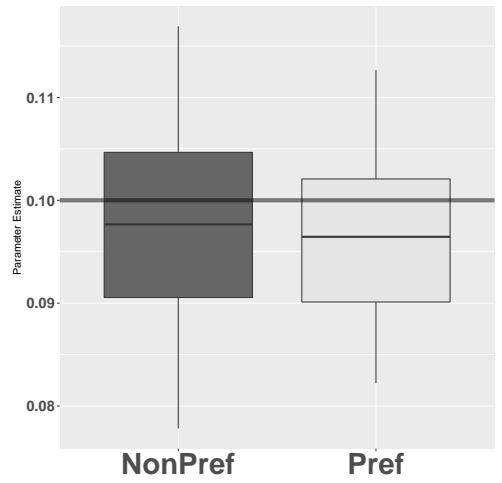

(d) Nugget variance $\left(\tau^{2}\right)$

Figure 8.3: Field parameter estimates over 50 preferentially simulated data sets with true parameter values marked as black lines. The abbreviations NonPref and Pref stand for the standard MLE (non-preferential) estimation and those using the preferential PCRW model of Section 8.3 respectively. 


\subsection{Simulation Experiments}

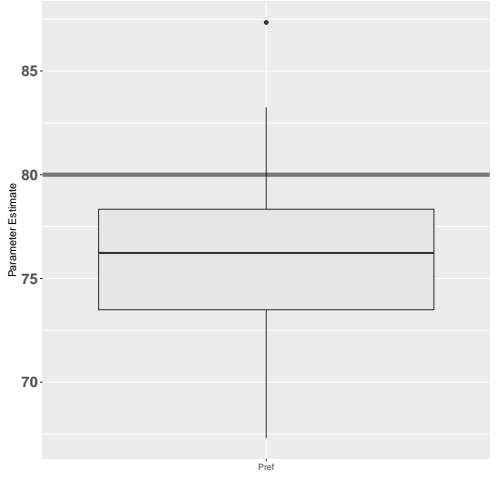

(a) $\alpha$

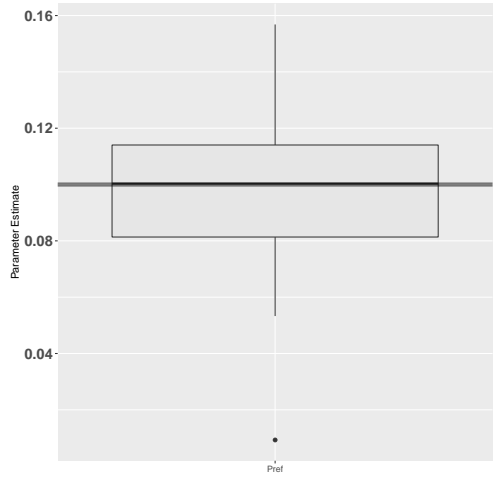

(b) $\sigma_{\beta}$

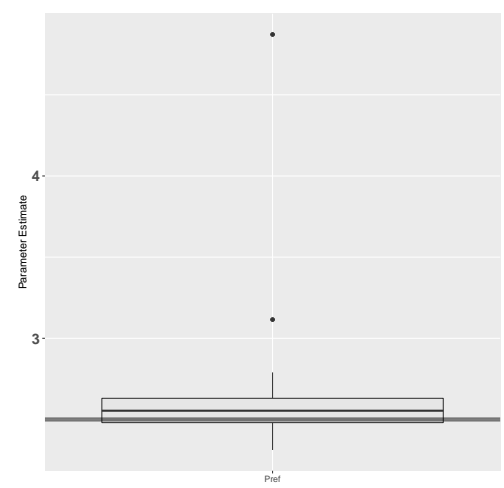

(c) Diffusion Variance $\left(\operatorname{Diag}\left(\boldsymbol{\Sigma}_{x}\right)\right)$

Figure 8.4: Movement parameter estimates over 50 preferentially simulated data sets with true parameter values marked as black lines. 


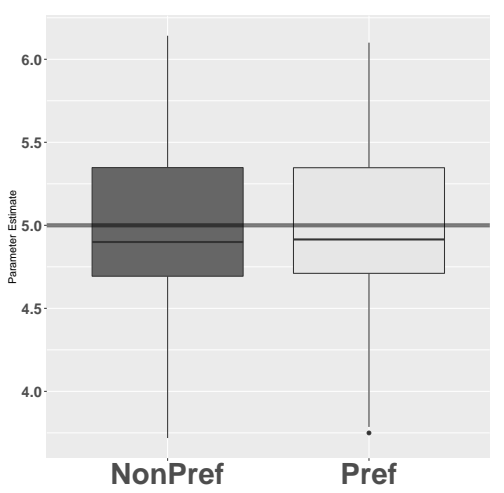

(a) Mean $(\mu)$

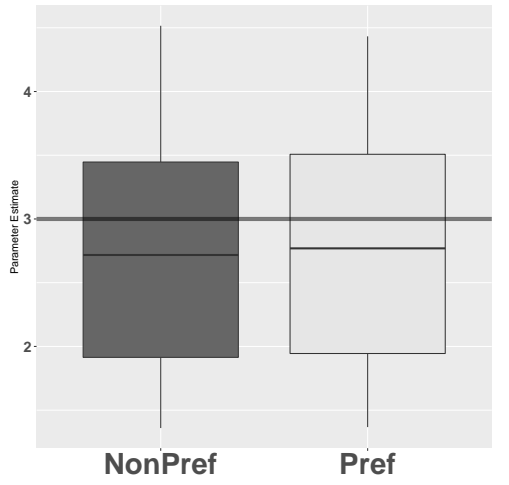

(c) Variance $\left(\sigma^{2}\right)$

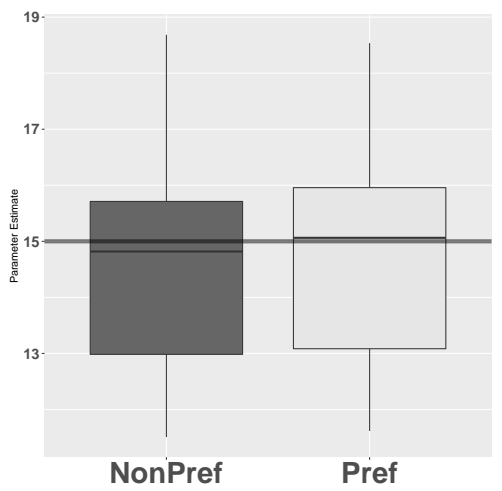

(b) Scale $(\phi)$

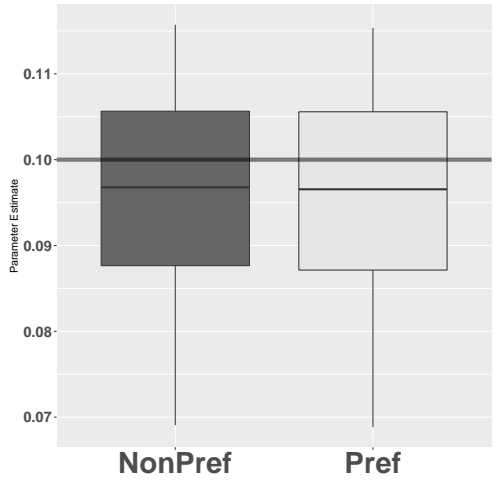

(d) Nugget variance $\left(\tau^{2}\right)$

Figure 8.5: Field parameter estimates over 50 non-preferentially simulated data sets with true parameter values marked as black lines. The abbreviations NonPref and Pref stand for the standard MLE (non-preferential) estimation and those using the PCRW model of Section 8.3 respectively. 


\subsection{Simulation Experiments}

for each location $i=1, \ldots, N$ on the prediction grid, where $N=26^{2}, \mathbf{S}_{j, i}$ and $\hat{\mathbf{S}}_{j, i}$ are the true and predicted temperatures respectively at location $\mathbf{x}_{i} \in \mathcal{D}$ for the $j$-th simulated date set. To assess the prediction methods whilst accounting for their variances, we again use ignorance scores as described in Section 5.3, where the target forecast in this case would be the true SST at that location. For each of the $j=1, \ldots, M=50$ preferential simulations we calculate the mean ignorance score (MIGN) of our latent field predictions as

$$
\operatorname{MIGN}_{j}=\frac{1}{N} \sum_{i=1}^{N}\left\{\frac{\left(\mathbf{S}_{j, i}-\hat{\mathbf{S}}_{j, i}\right)^{2}}{\hat{\sigma}_{j, i}^{2}}+2 \log \hat{\sigma}_{j, i}\right\} \quad j=1, \ldots, M,
$$

where $\hat{\sigma}_{j, i}^{2}$ is the prediction variance of $\hat{\mathbf{S}}_{j, i}$. This measure gives an indication of model performance for each simulation averaged over the entire domain. We also calculate location-specific ignorance scores, averaging the IGN of each location over the 50 samples, we call them location ignorance scores (LIGN):

$$
\operatorname{LIGN}_{i}=\frac{1}{M} \sum_{j=1}^{M}\left\{\frac{\left(\mathbf{S}_{j, i}-\hat{\mathbf{S}}_{j, i}\right)^{2}}{\hat{\sigma}_{j, i}^{2}}+2 \log \hat{\sigma}_{j, i}\right\} \quad i=1, \ldots, N .
$$

The LIGN gives an assessment of model prediction across each region of the domain.

To compare the "standard" predictions with the "preferential" ones we compute the corresponding differences in the above 3 measures:

$$
\begin{aligned}
\mathrm{RMSPE}_{i}^{\text {Diff }} & =\mathrm{RMSPE}_{i}^{\mathrm{P}}-\mathrm{RMSPE}_{i}^{\mathrm{NP}}, \\
\mathrm{MIGN}_{j}^{\text {Diff }} & =\mathrm{MIGN}_{j}^{\mathrm{P}}-\mathrm{MIGN}_{j}^{\mathrm{NP}}, \\
\mathrm{LIGN}_{i}^{\text {Diff }} & =\mathrm{LIGN}_{i}^{\mathrm{P}}-\mathrm{LIGN}_{i}^{\mathrm{NP}},
\end{aligned}
$$

where NP and $\mathrm{P}$ indicate the values of the scoring functions for the nonpreferential (standard) and preferential models, respectively.

The first panel in Figure 8.6 shows the values of $\mathrm{RMSPE}_{i}^{\text {Diff }}$ at each location, colouring the areas in blue for which this measure was negative, which correspond to regions where RMSPE for the predictions based on the preferential sampling model were better. Nearly all regions were predicted more accurately on average using the preferential sampling model, with only a small number of positive (red) locations.

The boxplot of the $M=50$ differences in MIGN is displayed in the second panel of Figure 8.6. Negative values of this difference imply that the $\mathrm{P}$ 


\subsection{Simulation Experiments}

model had a lower average ignorance score (over the region) for that particular simulation run. Again we see that the preferential sampling model performed better than standard methods across the majority of simulated data sets. This conclusion is further supported by the third panel in Figure 8.6 which shows LIGN $_{i}^{\text {Diff }}$ for each lattice prediction location. All locations had a smaller location ignorance score on average when using the preferential sampling model, which interestingly included the locations where point predictions were slightly inferior. This suggests that, even in this case, the prediction variances were considerably more accurate.

We also compare the scoring functions over the 50 non-preferentially sampled data sets to verify that, as expected, there was no qualitative difference between the results (in this case predictions) obtained using either method (the standard one that conditions on the locations, and the one based on the PCRW model). The results are displayed in Figure 8.7.

\subsubsection{Simulation 2}

In this section we conduct a simulation similar to the first, except with a simplified and potentially less preferential sampling system. The aim is to assess the impact of the latent field influencing movement speed, but not direction. We expect this to induce some sort of preferential effect, but correction in this case might be more difficult due to this being the first example of a sampling system that does not actually have a preferred region in the domain.

To begin we used the same 50 fields from simulation 1, themselves generated using a Matérn covariance function (2.4) with smoothness parameter $\kappa=2$, scale $\phi=15$, marginal variance $\sigma^{2}=3$ and a constant mean $\mu=5$. Again tracks were initialised at a location chosen uniformly at random over the 2-dimensional domain $[-150,150] \times[-150,150]$, and the first 20 positions of each animal were considered a burn-in period and discarded. We simulated 3 tracks on each of the 50 fields with each track consisting of 100 observations after the burn-in. The time between consecutive observations followed an exponential distribution with rate parameter $\lambda=1$.

In this case however we altered the form of the function $\phi(\cdot)$ from $(8.9)$ to

$$
\phi(\mathbf{X}, S)=\alpha S(\mathbf{X}) .
$$

This generated movement where the preferential element was somewhat reduced since $\phi$ now only adjusts the speed of movement, rather than the direction as it had done previously. We wish to investigate the effect of this, 


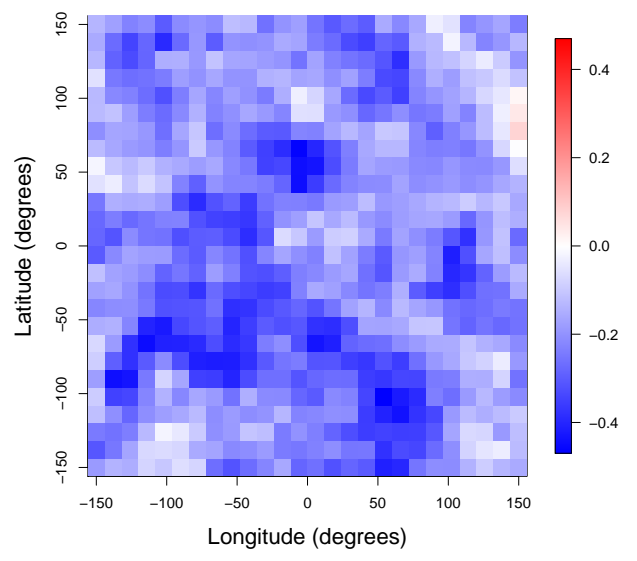

(a) $\operatorname{RMSPE}_{i}^{\text {Diff }}$

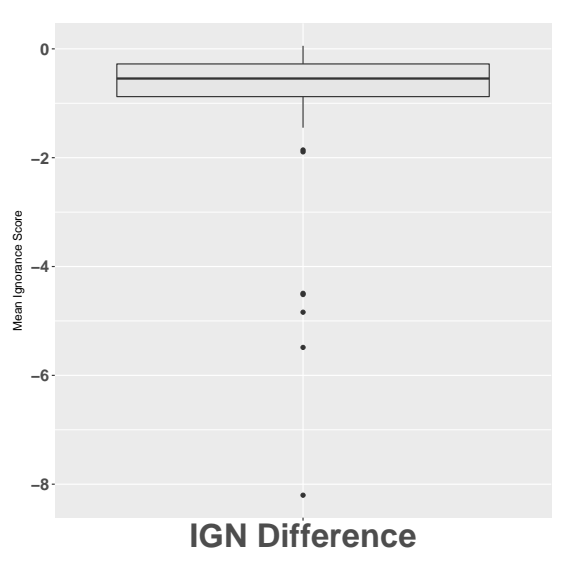

(b) $\mathrm{MIGN}_{j}^{\text {Diff }}$

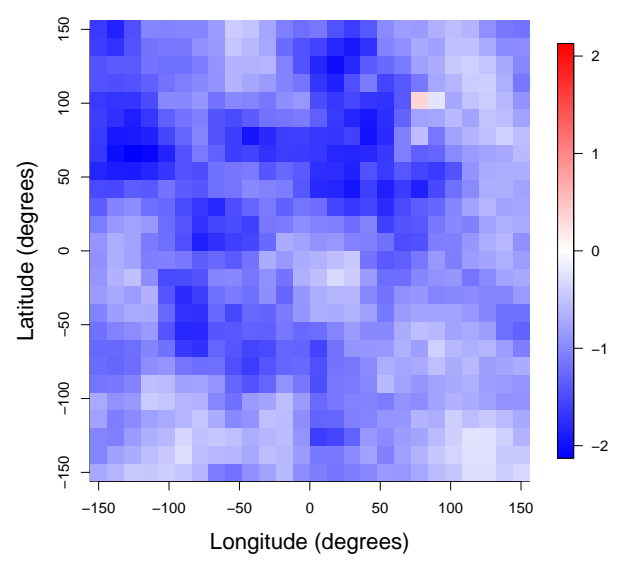

(c) $\operatorname{LIGN}_{i}^{\text {Diff }}$

Figure 8.6: Comparison of root-mean-square prediction error (RMSPE) difference, mean ignorance score (MIGN) difference and location ignorance score (LIGN) difference respectively across 50 preferentially simulated data sets. 


\subsection{Simulation Experiments}

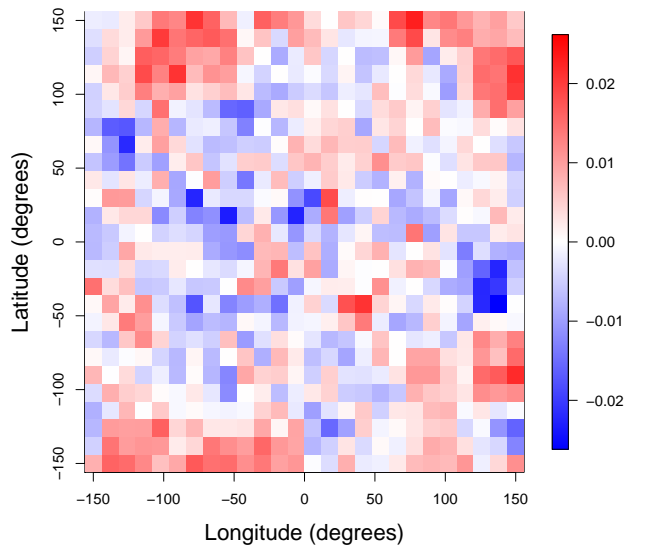

(a) $\operatorname{RMSPE}_{i}^{\text {Diff }}$

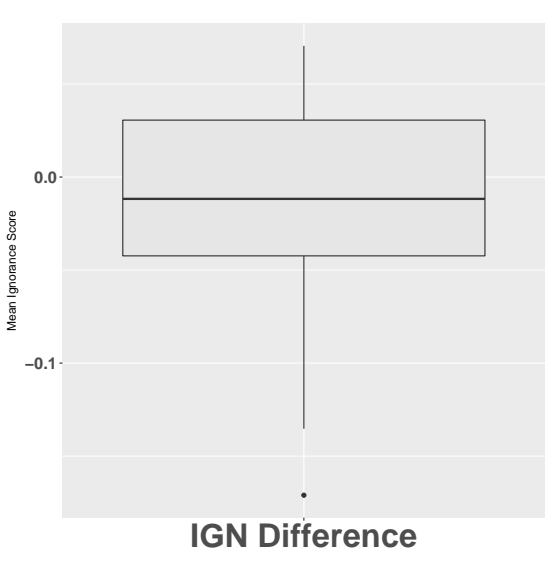

(b) $\mathrm{MIGN}_{j}^{\text {Diff }}$

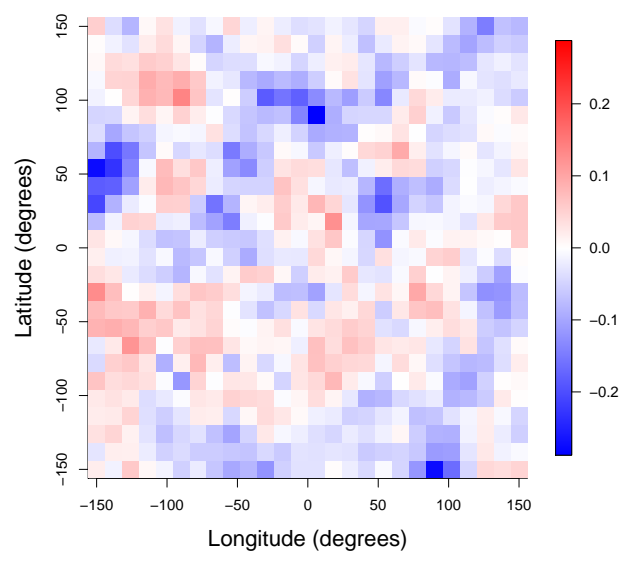

(c) $\operatorname{LIGN}_{i}^{\text {Diff }}$

Figure 8.7: Comparison of root-mean-square prediction error (RMSPE) difference, mean ignorance score (MIGN) difference and location ignorance score (LIGN) difference respectively across 50 non-preferentially simulated data sets. 


\subsection{Simulation Experiments}

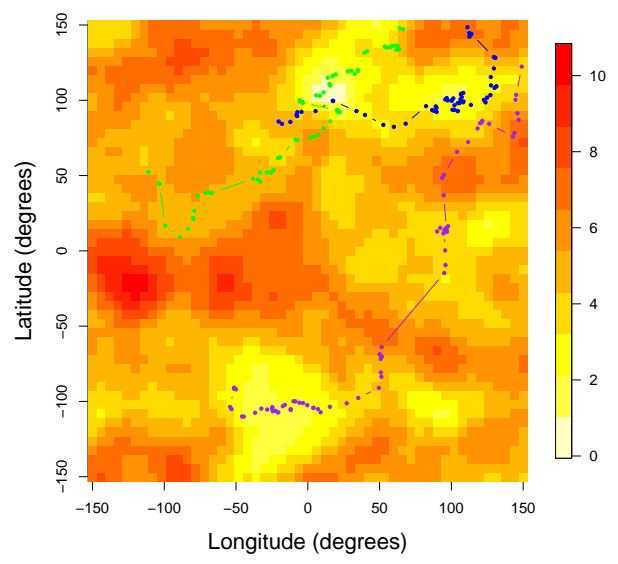

Figure 8.8: Example of a simulated data set of 3 tracks generated using the field and movement parameters defined in simulation 2 , resulting in a small preferential sampling effect.

if any, on parameter estimation and prediction across both the standard and fully specified models.

For the movement model we set $\boldsymbol{\Sigma}_{x}=2 \mathbf{I}_{2}, \sigma_{\beta}=0.1, \alpha=0.8$ and initiated the behavioural states at $\beta_{1}=0$. This model means that the mammal will move quicker at higher temperatures and slower in lower temperature regions, hence it may induce a negative bias in the mean parameter estimates and spatial predictions. An example of a single track can be seen in Figure 8.8 which uses the same latent field $\mathbf{S}$ as the example in Figure 8.2 .

\section{Parameter Estimates}

We begin by comparing field parameter estimates between the standard model and the preferential model implemented using TMB. We compare field parameters in Figure 8.9 which look very similar across the two methods, even though the mean parameter was regularly underestimated. The scale and variance estimates were also slightly underestimated by both methods, appearing similar to the non-preferential estimates from the preferentially sampled data in simulation 1 shown in Figure 8.3. This suggests that the form of $\phi(\cdot)$ may have introduced preferential movement to an extent similar to that in simulation 1 . However, there appeared to be no correction using the fully specified model in TMB. Movement parameters were accurately 


\subsection{Simulation Experiments}

estimated which we exclude for space.

\section{Prediction}

To compare predictions between ordinary kriging with the standard model estimates as plug-in parameters and the mode of $[\mathbf{S} \mid \mathbf{Y}, \mathbf{X}]$ from TMB, we compare RMSPE $E_{i}^{\text {Diff }}$, MIGN $_{j}^{\text {Diff }}$ and LIGN $_{i}^{\text {Diff }}$ as defined in (8.16).

The first panel in Figure 8.10 compares RMSPE ${ }_{i}^{\text {Diff }}$ across all 50 simulations for prediction locations $i=1, \ldots, 676$. Positive values of this scoring function imply that the preferential model had larger RMSPE's on average at that particular lattice location in comparison to ordinary kriging. In this case it seems as though the two models perform similarly, with no obvious improvement when using our PCRW model. Note also that the scale goes from -0.022 to 0.022 which shows that the difference between the two methods is minor compared to the preferential example in simulation 1 , that had a scale between -0.42 to 0.42 . This plot looks extremely similar to the non-preferential example in simulation 1 which had a very similar range for $\mathrm{RMSPE}_{i}^{\text {Diff }}$, shown in Figure 8.7.

The second panel in Figure 8.10 compares MIGN $_{j}^{\text {Diff }}$ for each of the $j=1, \ldots, 50$ data sets. This measure is an average ignorance score across all locations for each data set. This proves to be a much more interesting case. Although the RMSPE's were very similar, there appears to be a far larger tail to the distribution of values on the negative end of the difference. This non-symmetric distribution of values is similar to the preferential example in simulation 1, except that it centers on 0 . The non-preferential example in simulation 1 also centered on 0 but was more symmetric, with the preferential model rarely performing markedly better than the standard model. In this case it appears that the MIGN's were fairly equivalent across the two methods for the majority of simulations, but there were a substantial amount of cases in which a significantly superior MIGN was obtained using the preferential model.

Finally we compare LIGN in the final panel of Figure 8.10. In this case we plot $\operatorname{LIGN}_{i}^{\text {Diff }}$ for prediction locations $i=1, \ldots, 676$. Again there appears little difference between the two prediction methods, with the results look similar to the non-preferentially sampled results in the previous simulation.

The results in this simulation were somewhat inconclusive as to the effect of preferential sampling in this case. Unlike all other examples in this thesis, the sampling process was not attracted to particular values of the latent field, but it was still influenced by it, as can be seen in the underestimation of the mean parameter in Figure 8.9. However it appears that 


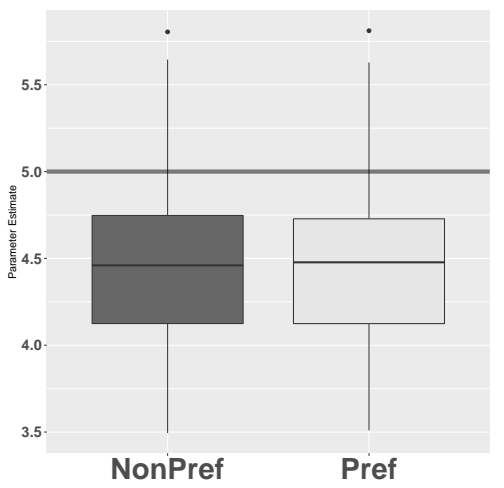

(a) Mean $(\mu)$

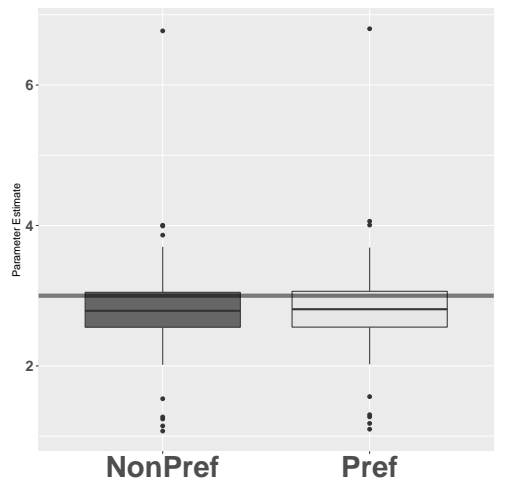

(c) Variance $\left(\sigma^{2}\right)$

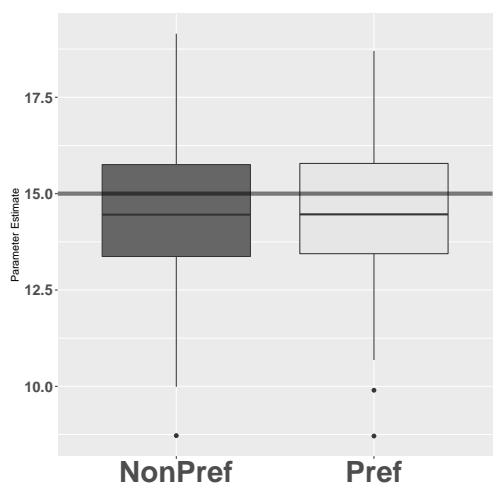

(b) Scale $(\phi)$

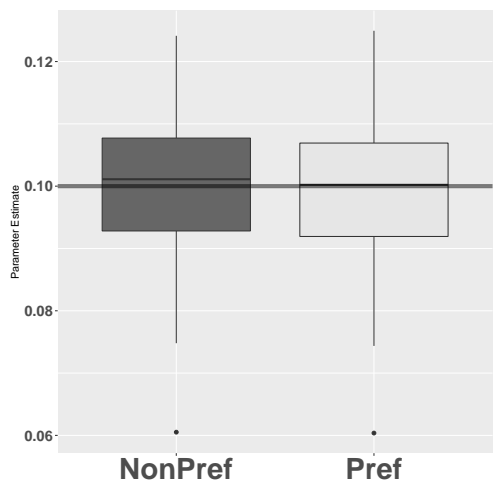

(d) Nugget variance $\left(\tau^{2}\right)$

Figure 8.9: Field parameter estimates over 50 data sets with true parameter values marked as black lines for simulation 2 . The abbreviations NonPref and Pref stand for the standard MLE (non-preferential) estimation and those using the PCRW model of Section 8.3 respectively. 


\subsection{Simulation Experiments}

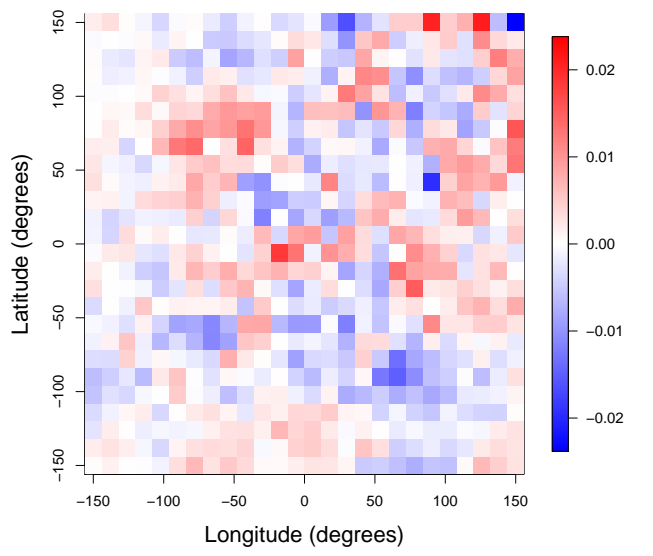

(a) $\operatorname{RMSPE}_{i}^{\text {Diff }}$

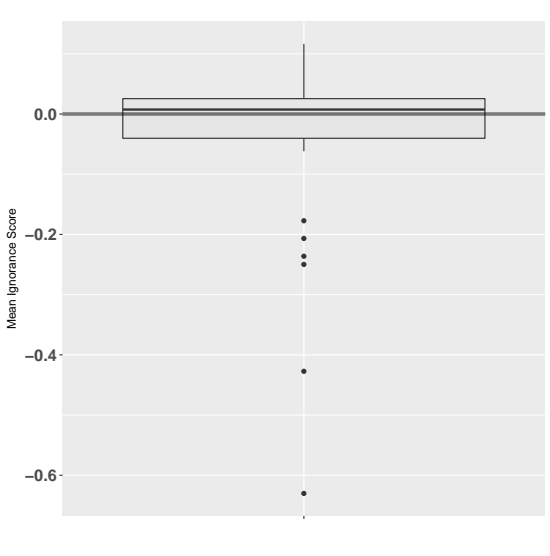

(b) $\mathrm{MIGN}_{j}^{\text {Diff }}$

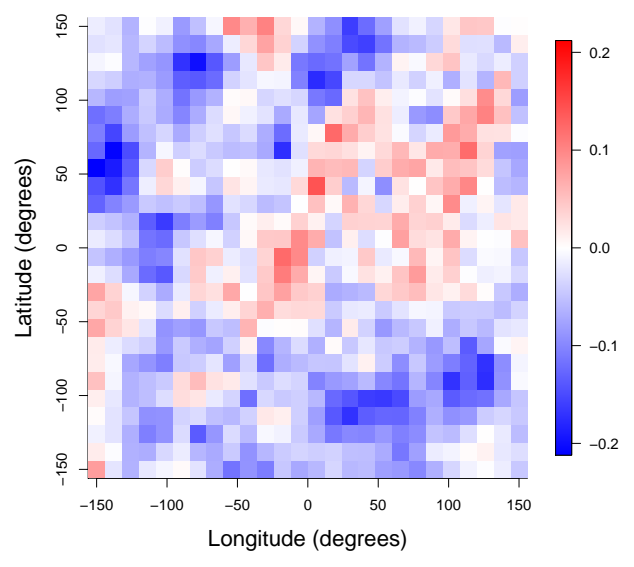

(c) $\operatorname{LIGN}_{i}^{\text {Diff }}$

Figure 8.10: Comparison of root-mean-square prediction error (RMSPE) difference, mean ignorance score (MIGN) difference and raw values of the location ignorance score (LIGN) difference respectively across 50 data sets from simulation 2 . 
there was little to no correction in parameter estimates and little change in the point prediction. On the other hand there is evidence that the preferential model may still improve prediction. This can be seen when comparing MIGNs in Figure 8.10, which had a skewed distribution with some large gains made in ignorance scores using the preferential model. It is difficult to say whether this was a consequence of the PCRW model specification which inherently has larger prediction errors than the kriging model, which may improve ignorance scores in poorly prediction areas. This increase in prediction error is due to an increased number of parameters included in the predictive distribution of the full model. However, this factor would also mean that for accurate point prediction that the preferential model would have inferior (larger) ignorance scores, so it was unlikely to be the only reason. Furthermore, since this ignorance score pattern was not evident in the non-preferential example of simulation 1, it appears that including the movement model even when the preferential sampling does not inherently prefer particular regions of the latent field may be of use in the predictions.

\subsection{Real Data Example}

In this section we analyse Southern Indian ocean temperatures at a depth of 6 meters, which we will be calling sea surface temperatures (SSTs), using CTD (Conductivity-Temperature-Depth) sensor data from tags attached to Elephant Seals. These data were collected and made freely available as part of the MEOP (Marine Mammals Exploring the Oceans Pole to Pole) database (Roquet et al., 2014a b, 2013) and is described in Section 8.2.1. We analyse data restricted between the months of July to September 2012 to reduce the impact of varying ocean temperatures across time and this subset consists of 13 separate tracks with 3369 observations in total, which can be seen in Panel (a) of Figure 8.11.

Since these data are relatively near to the South Pole, there is a large change in true distance between latitudinal and longitudinal degrees over the domain. For example, we can see in Panel (a) of Figure 8.11 that the change in latitude is not constant in distance between $-50^{\circ}$ and $-70^{\circ}$. This is due to the difficulties of representing locations from a 3-dimensional sphere on a 2-dimensional surface, which is of particular importance near the poles. One option is to use the Haversine formula to calculate the greatcircle distance between two points in the latitude/longitude space (Robusto, 1957). However, as is discussed by Gneiting (2013); Jeong and Jun (2015) among others, using the Matérn covariance along with great circle distances 
requires the use of a non mean-square differentiable processes $(0<\kappa \leq 0.5)$, since the Matérn class is not isotropic otherwise.

To more accurately represent distance over our domain but remaining in the Euclidean space, we transformed the sampling locations. Our transformation used a scaled version of the Universal Transverse Mercator (UTM) projection (zone 43) which can be seen in Panel (b) of Figure 8.11. This transformation provides us with a more accurate representation of distance than using raw latitudes and longitudes, whilst remaining in the 2dimensional Euclidean space so to retain the isotropic and mean-square differentiable properties of our correlation model. The values of our scale have no real-world interpretation, other than to provide more realistic scaled distances between sampled locations.

We compare both the parameter estimates and consequent field predictions using the PCRW model described in Section 8.3.1, with those obtained from a standard geostatistical model in which the sampling locations were considered independent of the temperature field. In order to explore the sampling distribution of the parameter estimators for the SST field obtained with each model, we ran a re-sampling experiment. We randomly sampled 30 observations from each of the 13 tracks and estimated the parameters on the sub-sampled data. This was repeated 50 times, with the resulting estimates displayed in Figures 8.12 and 8.13 .

We note that the difference between the two sets of estimates is very small in the mean, the scale and nugget parameter estimates. However, there was a small reduction in the variance parameter estimate using the PCRW model. The consistently positive $\hat{\alpha}$ 's suggest that there was a tendency of the animals to move towards cooler regions according to our model.

For predictive assessment of the ocean temperatures obtained from marine mammal tags we compare our model predictions with monthly average temperature fields obtained via the Simple Ocean Data Assimilation ocean/sea ice reanalysis (SODA) (Carton and Giese, 2008), specifically the SODA version 3 (SODA3) reanalysis (Carton et al., 2018), which uses all temperature and salinity profiles from the World Ocean Database. This data is available for depths between 5 and 5000 meters below the surface at a spatial resolution of 0.5 degrees latitude and longitude. Data is available from January 1980 to present.

We compare our predictions with the monthly average ocean temperature field for August obtained at a depth of 5 meters below sea level via SODA3. This data can be seen in the first panel of Figure 8.14. Note that we compare the predictions at $N=505$ locations which is a subset of the square lattice. These locations were chosen as those on the original $26 \times 26$ 


\subsection{Real Data Example}

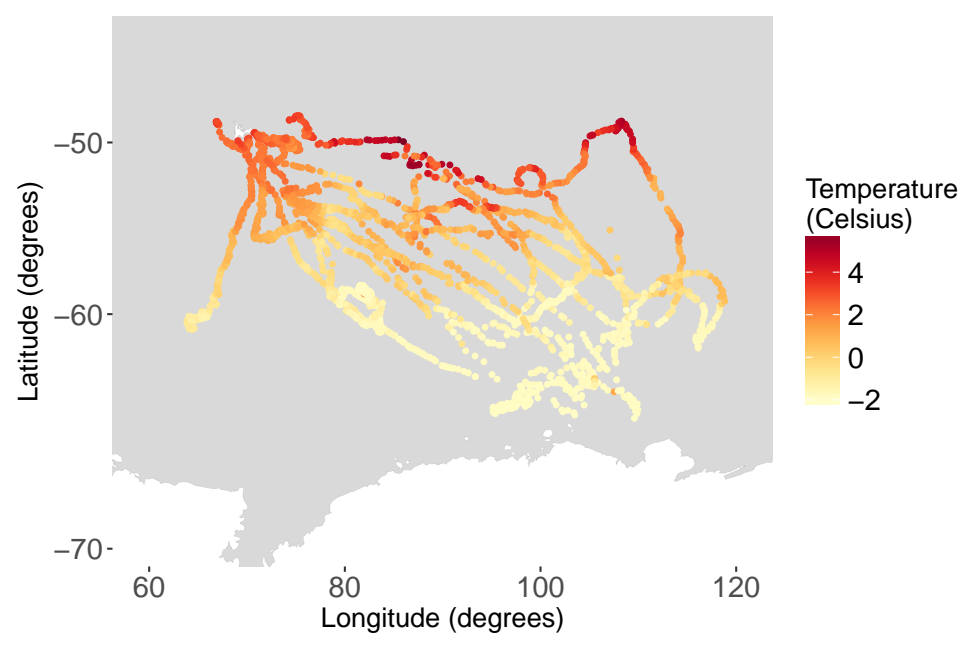

(a) Raw Data

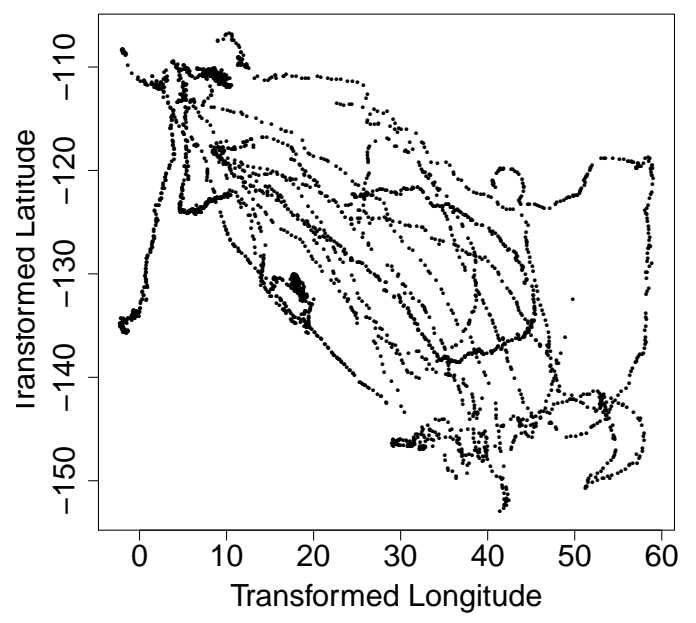

(b) Transformed Coordinates

Figure 8.11: Plot all 3369 observations from the subset of Southern elephant seal data analysed in Section 8.5. The first observation was taken on July 1st 2012 and the final observation on 30th September 2012. Panel (a) shows raw latitude and longitude plots with corresponding temperatures whilst Panel (b) shows the corresponding transformed locations using a scaled version of the Universal Transverse Mercator (UTM) projection (zone 43). Map tiles by Stamen Design, under CC BY 3.0. Data by OpenStreetMap, under ODbL. 


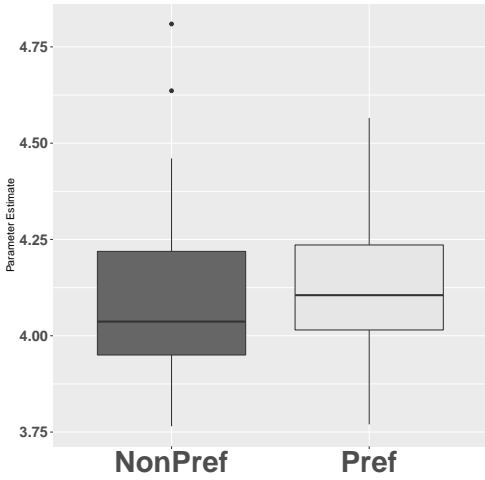

(a) Mean $(\mu)$

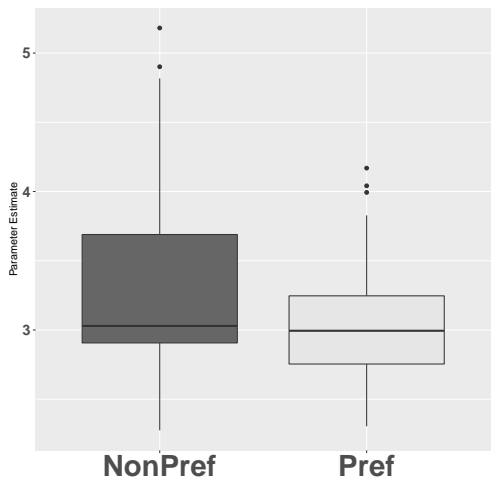

(c) Variance $\left(\sigma^{2}\right)$

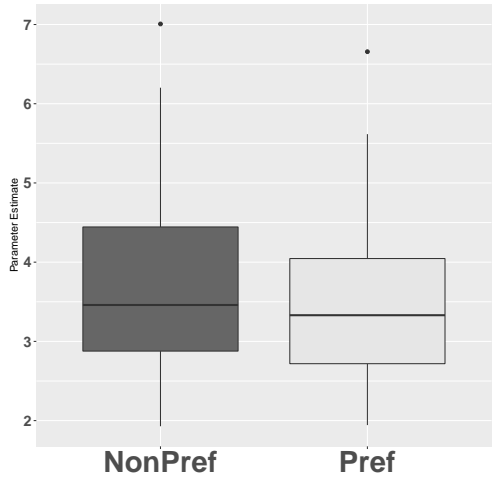

(b) Scale $(\phi)$

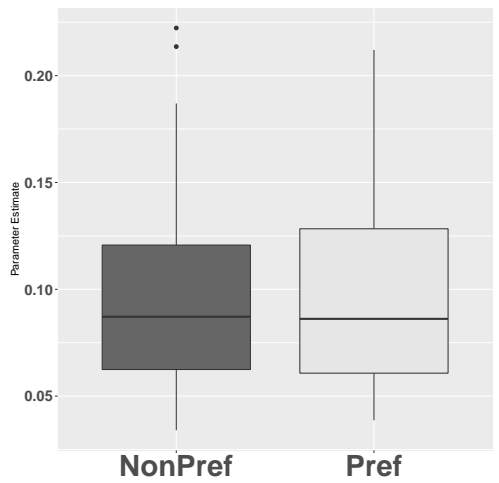

(d) Nugget variance $\left(\tau^{2}\right)$

Figure 8.12: Comparison between preferential and standard MLEs for the field parameters over 50 data sets consisting of a sub-sample of 13 tracks with 30 observations each. 


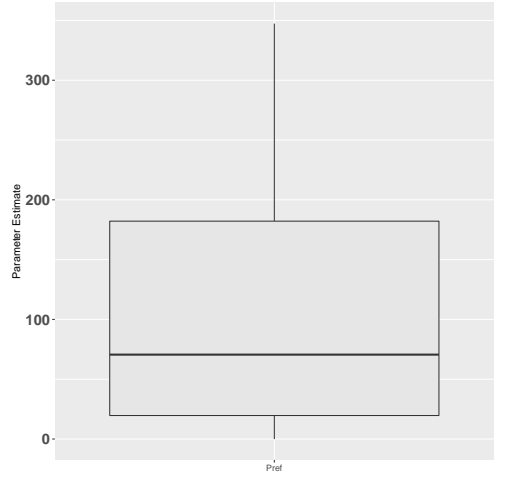

(a) $\alpha$

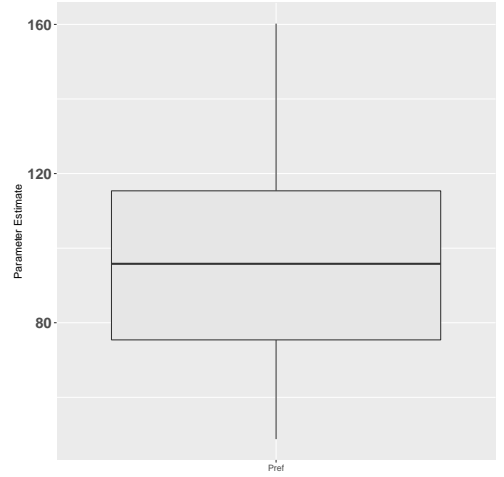

(b) $\sigma_{\beta}$

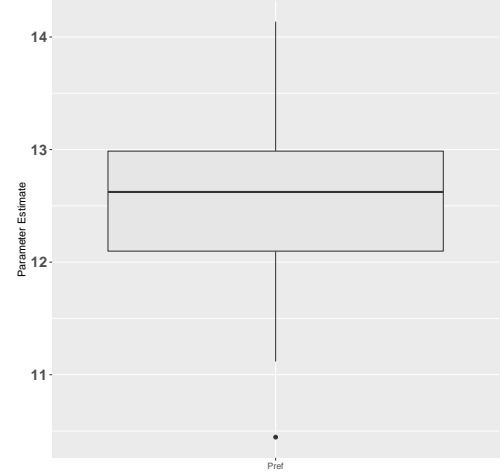

(c) Diffusion Variance $\left(\operatorname{Diag}\left(\boldsymbol{\Sigma}_{x}\right)\right)$

Figure 8.13: Movement parameter estimates using the preferential model over 50 data sets consisting of a sub-sample of 13 tracks with 30 observations each. 
lattice points which were close enough to sampling locations to obtain kriging predictions that did not simply tend to the mean trend parameter $\hat{\mu}$. The locations on the lattice which were ignored are shown in grey. The average of the non-preferentially predicted fields across 50 data sets using the standard MLE parameter estimates as full set of plug-in parameters for ordinary kriging can be seen in the fourth panel, which is compared against the average field obtained via the mode of the intractable preferential distribution $[\mathbf{S} \mid \mathbf{X}, \mathbf{Y}]$ provided by TMB in the third panel.

To compare point predictions we consider the quantiles of the difference between the two prediction methods on each of the data sets at each prediction location. In other words we consider the quantiles of each coordinate of the vector $\mathbf{D}=\left(D_{1}, \ldots, D_{N}\right)$ for $i=1, \ldots, 505$ such that

$$
D_{i}=\left(\hat{S}_{1, i}^{P}-\hat{S}_{1, i}^{N P}, \ldots, \hat{S}_{50, i}^{P}-\hat{S}_{50, i}^{N P}\right)^{T},
$$

where $\hat{S}_{j, i}^{P}$ is the prediction at location $i$ in simulation $j$ using the preferential model and $\hat{S}_{j, i}^{N P}$ the equivalent using the non-preferential model.

We plot the $50 \%$ quantile of $\mathbf{D}$ in the second panel of Figure 8.14. As we would expect with the positive $\alpha$ estimates, most areas further from sampling locations had an increased SST prediction using the PCRW model. Interestingly however, the PCRW model actually tended to decrease SST prediction in certain areas of the West and North-East predictive region.

To assess the prediction accuracy compared to the SODA3 data set we consider ignorance scores and RMSPE's in the same manner as in Section 8.4. We plot the difference in RMSPE, MIGN and LIGN defined in (8.16) in Figure 8.15. The first panel shows that the PCRW model tended to reduce RMSPE in comparison to non-preferential prediction in the Northern regions, whilst underperforming in areas of the South. This suggests that a linear trend in the mean function might be a more suitable model, something which we investigate in Section 9.2. However, the LIGN in the third panel shows improved ignorance scores in general using the preferential model. The clearest indication of superior prediction performance is through the second panel of Figure 8.15 which shows nearly all MIGN's across the 50 data sets were negative, indication of a prediction improvement using the PCRW across the vast majority of cases.

To summarise, the PCRW model applied using TMB showed only small differences in mean parameter estimation when compared to the naive method, but the model identified possible tendency of sampler movement towards the cooler regions. This translated to many areas of increased SST prediction when using the preferential model in spatial prediction, but not across the 


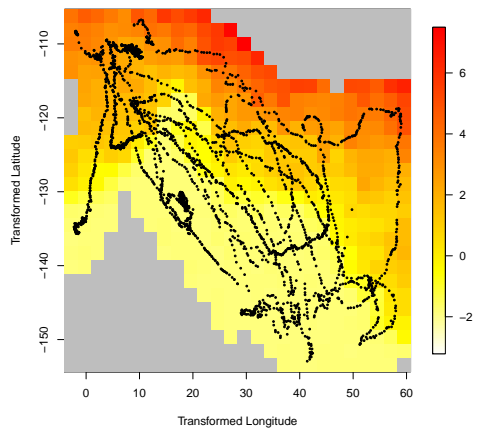

(a) SODA3 Temperature Field

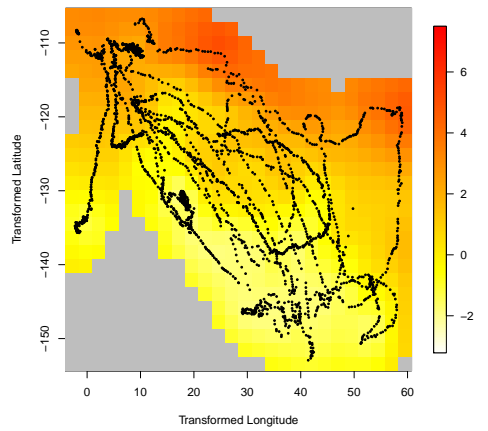

(c) Preferential Temperature Field (d) Non-preferential Temperature
Field

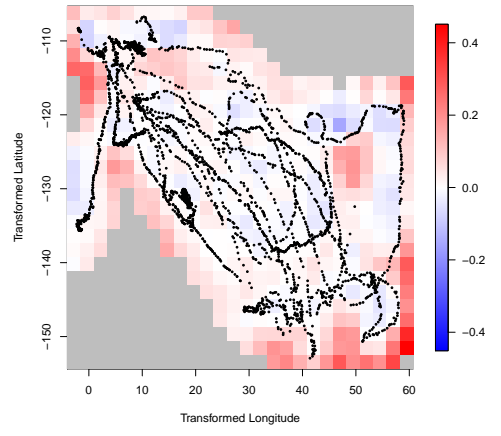

(b) Median of Prediction Difference

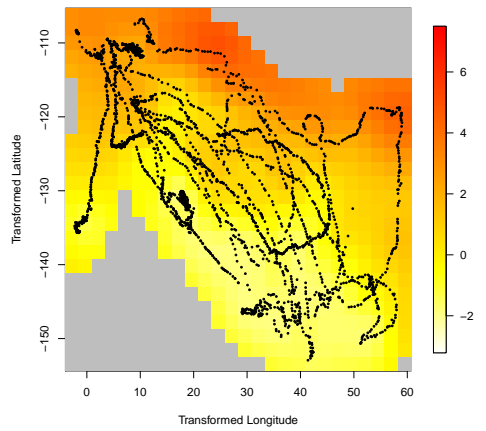

Figure 8.14: Panel (a): Monthly average field $\left({ }^{\circ} \mathrm{C}\right)$ obtained via the Simple Ocean Data Assimilation ocean/sea ice reanalysis version 3 (SODA3) analysis (Carton et al., 2018). Panel (b): Median of prediction difference between preferential and non-preferential models $\left({ }^{\circ} \mathrm{C}\right)$. Positive values imply that the preferential model tends to increases temperature prediction at that location and negative the opposite. Panels (c)-(d): Comparison of predicted fields using preferential and non-preferential models $\left({ }^{\circ} \mathrm{C}\right)$. 


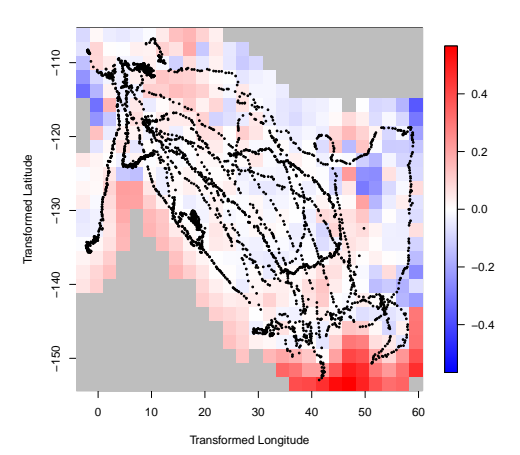

(a) $\operatorname{RMSPE}_{i}^{\text {Diff }}$

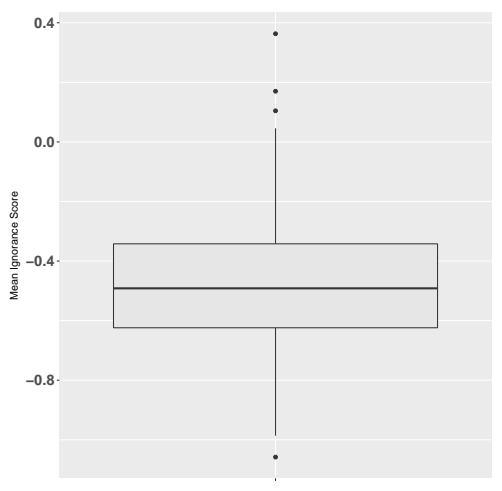

(b) $\mathrm{MIGN}_{j}^{\text {Diff }}$

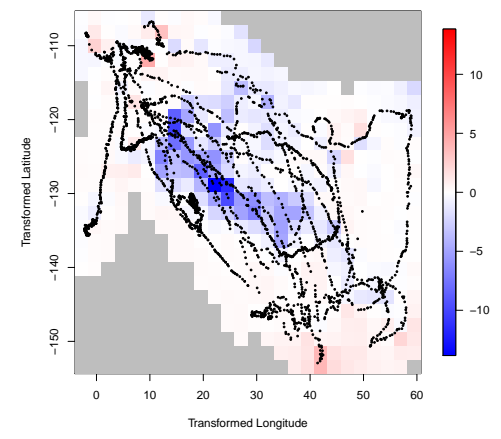

(c) $\operatorname{LIGN}_{i}^{\text {Diff }}$

Figure 8.15: Comparison of root-mean-square prediction error (RMSPE) difference, mean ignorance score (MIGN) difference and location ignorance score (LIGN) difference respectively across 50 simulated data sets between preferential and non-preferential temperature predictions. 
entire spatial region. Small changes in SST prediction as shown by our model may be of huge importance when dealing with the subtleties of ocean temperature change. Although this simulation used a simplistic relationship between the sampler and the process being modelled, our results suggest that developing more realistic models for this type of data can improve the resulting statistical inference about our ocean climates. 


\section{Chapter 9}

\section{Preliminary Extensions}

In this chapter we propose some extensions to the preferential sampling model framework proposed in this thesis. The work here differs from that in the future work Chapter 10, in that we will show initial results from simulation studies investigating the methods, even though they are net yet complete. We begin with an example and discussion on how to extend the SST analysis to ocean temperatures at greater depths in Section 9.1 and introduce non-constant trends to the mean function in Section 9.2.

\subsection{Extending Ocean Temperatures to New Depths}

A natural extension of the research on the preferential sampling of ocean temperatures presented in this thesis is to consider data at any depth. Although SST analysis is a useful first step, an important application of these methods would be to aid in mapping the water temperatures at depths unobservable by satellites. Theoretically, in analyses at specific depths little would change in comparison to the SST data we considered in Chapter 8 , however the location accuracy underwater is reduced due to location measurements only being available at the surface. The Elephant seal data used in this thesis consists of measurements collected during the deepest dive every 6 hours, with the water temperature and depth recorded regularly on the ascension to the surface, and the ARGOS location estimate taken once it reaches the surface (after the dive). These ascents are quasi-vertical (Roquet et al., 2013) so shallow depths should not be recorded too far from the surface for this particular mammal. With advances in technology over time it is likely that more temperature measurements will be collected with the corresponding accelerometry data, although at this time we could not find freely available data which had both. With tags capable of collecting both oceanographic and accelerometry data, underwater location estimates could be inferred based on the accelerometer data using methods such as the dead reckoning algorithm (Wilson and Wilson, 1988) and ocean temperature at 
specific depths analysed to build a water temperature profile more accurately. We give an example of how this might look using an alternative subset of the Roquet et al. (2014b) data set described in Section 4.5.1.

The data set we analyse in this example is shown in Panel (a) of Figure 9.1 and consists of 2264 observations taken between July 1 st 2012 and 30th September 2012 from 9 tracks in total. We used the water temperature taken at 50 meters below sea level, but the locations are those recorded at the surface following the dive using ARGOS. As mentioned previously, this might contain important measurement error which is why this application needs further research, but we try to reduce the impact of this by considering data at somewhat shallow depths. As in Section 8.5, to more accurately represent distance over our domain we transformed the sampling locations using a scaled version of the Universal Transverse Mercator (UTM) projection (zone 43) which can be seen in Panel (b) of Figure 9.1. Again, we assess prediction by comparison with model outputs from the Simple Ocean Data Assimilation ocean/sea ice reanalysis (SODA) (Carton and Giese, 2008), but for a depth of 50 meters.

We follow the example in Section 8.5 by comparing both the parameter estimates and consequent field predictions using the PCRW model described in Section 8.3 with those obtained from a standard geostatistical model in which the sampling locations are considered independent of the temperature field. Again we explore the distribution of the parameter estimates for the temperature field obtained from each model by running a re-sampling experiment. We randomly sampled 30 observations from each of the 9 tracks and estimated the parameters on the sub-sampled data. We repeated this 50 times and show the estimates for the constant mean parameter $\mu$, scale $\phi$, variance $\sigma^{2}$ and the preferential parameter $\alpha$ in Figure 9.2. The remaining field and movement parameters can be found in Figures D.1. D.2 in Appendix D.

There was a noticeable increase in the estimates of $\mu$ using the PCRW model, which corresponds to the positive estimates of $\alpha$. The $\alpha$ estimates follow a similar pattern observed in the SST analysis in Section 8.5, but are actually larger in general in this case. This suggests movement of the samplers towards cooler regions is more extreme, which is likely the reason behind the larger difference in $\mu$ estimates between the two models. There was little change in the scale $(\phi)$ and variance $\left(\sigma^{2}\right)$ estimates when using the preferential model, something which was also observed in the previous SST simulation.

We assess prediction between the two models in comparison to SODA3 in Figure 9.3, where as in the previous section, we ignore prediction locations 


\subsection{Extending Ocean Temperatures to New Depths}

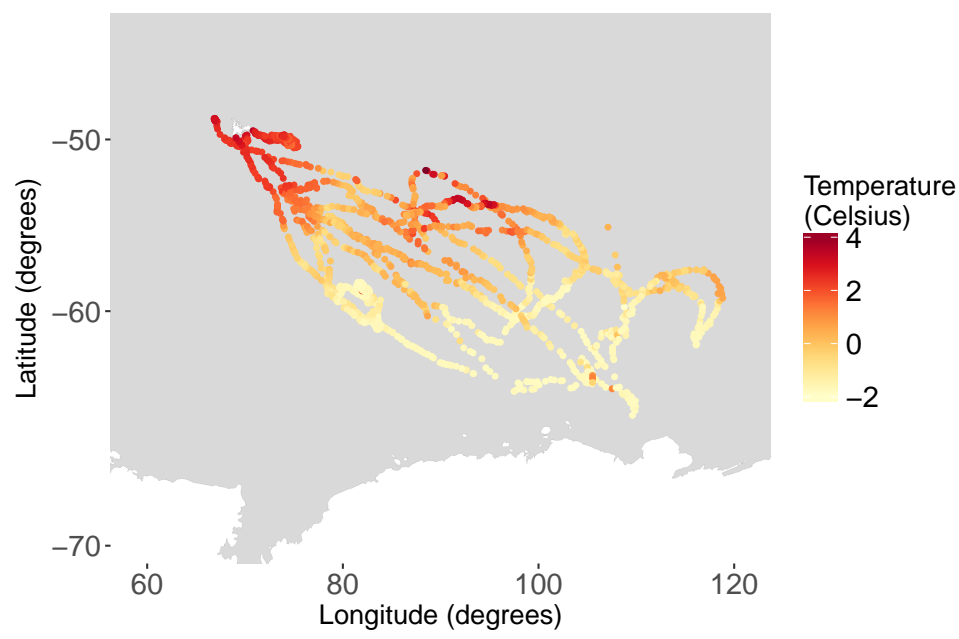

(a) Raw Data

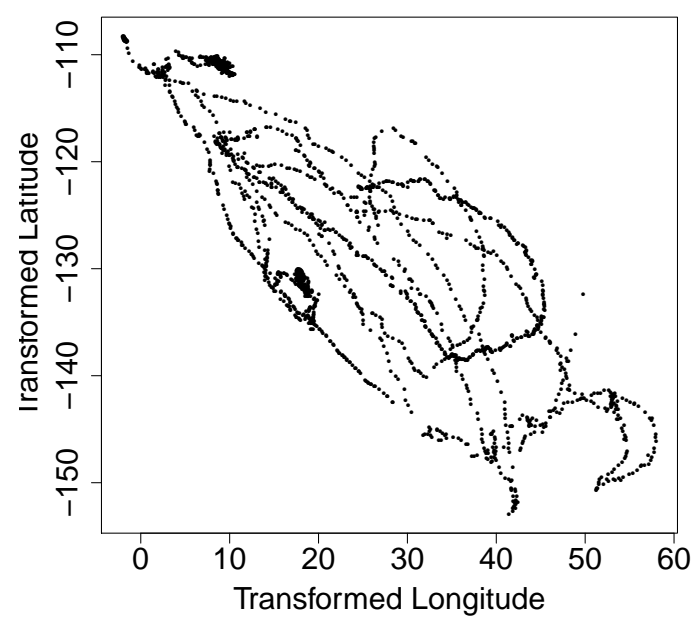

(b) Transformed Coordinates

Figure 9.1: Plot all 2264 observations from the underwater data set taken at 50 meters below sea level. The first observation was taken on July 1st 2012 and the final observation on 30th September 2012. Panel (a) shows raw latitude and longitude plots with corresponding temperatures whilst Panel (b) shows the corresponding transformed locations using a scaled version of the Universal Transverse Mercator (UTM) projection (zone 43). Map tiles by Stamen Design, under CC BY 3.0. Data by OpenStreetMap, under ODbL. 


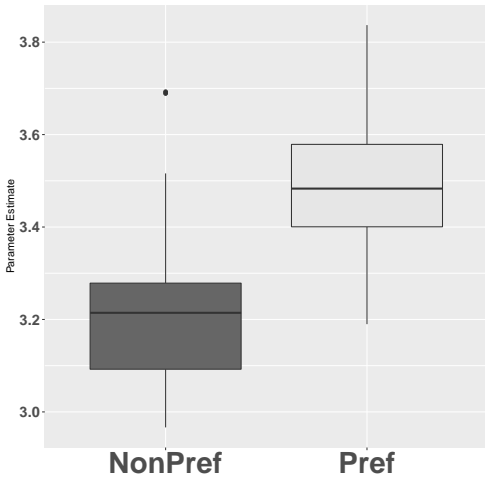

(a) Mean $(\mu)$

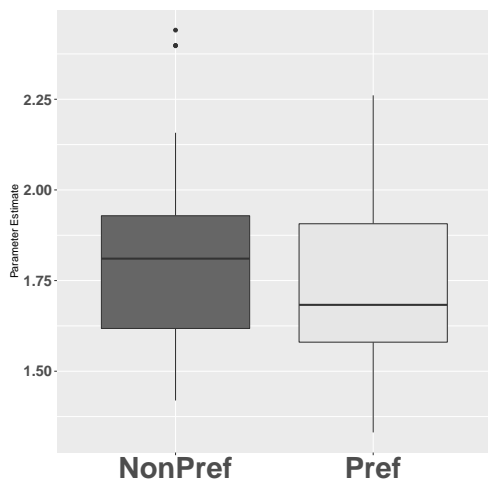

(c) Variance $\left(\sigma^{2}\right)$

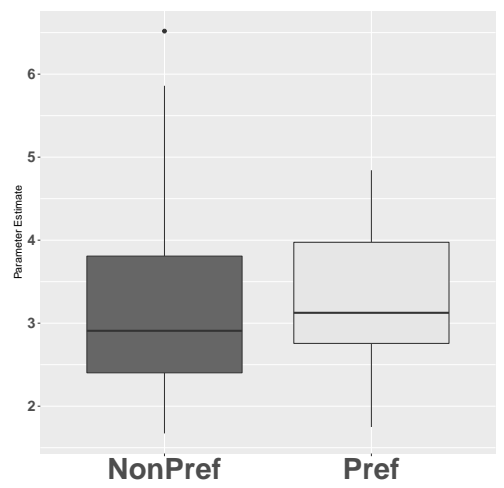

(b) Scale $(\phi)$

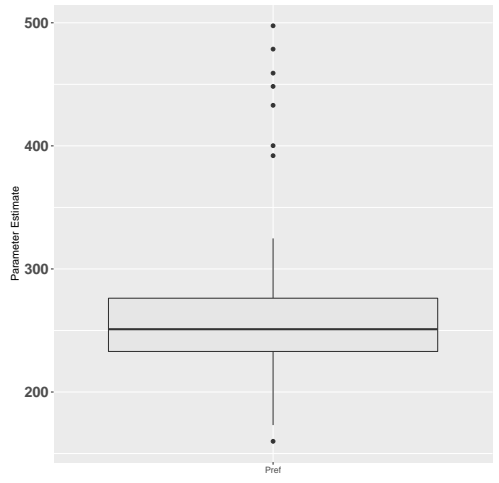

(d) $\alpha$

Figure 9.2: Panels (a)-(c): Parameter estimate comparisons for $\mu, \phi$ and $\sigma^{2}$ using the non-preferential and preferential model over 50 data sets consisting of a sub-sample of 9 tracks of 30 observations each. Panel (d): Parameter estimates for the preferential parameter $\alpha$ in the preferential model. 
that are far from sampled locations. This results in $N=392$ prediction locations. The SODA3 model output can be seen in Panel (a), whilst the preferential predictive surface is shown in Panel (b). In Panel (c) we compare the $50 \%$ quantile of differences $\mathbf{D}$ as defined in (8.17). As we would expect due to the positive $\hat{\alpha}$ 's the preferential model generally increased the water temperature predictions in comparison to the standard method. We also compare the difference in RMSPE, MIGN and LIGN defined in (8.16). There was an improvement in RMSPEs when using the PCRW model, particularly in the Northern regions. However, the MIGN and LIGN are more comparable for the preferential model in comparison to standard methods. It should be noted that two prediction locations $((19.44,-133.34),(9.68,-109.60))$ were ignored for the prediction comparison due to inadequate prediction from both models compared to SODA3 outputs. The difference between the two models appears to be even larger than in the SST example from Section 8.5, so there is evidence that there may still be gains to made using preferential models at lower depths.

However, as before we should be cautious in our interpretations of these results. The predictions are compared with model outputs at a depth of 50 meters, which is not as accurate as the data at a depth of 5 meters. Furthermore, as we discussed earlier in this section, the locations are less accurate since we are using the ARGOS locations obtained at the end of the dive from which the temperature data is obtained. Having said this, the analysis in this section shows how one could approach mapping horizontal layers of underwater temperatures in the same way as SST with little change to the approach. The main work in this case would be improving the location estimation accuracy and judging how best to compare the model predictions to those from projects such as SODA.

\subsection{Non-Constant Mean Trend}

In this thesis we have been assuming a constant mean trend $\mu$ for the field of interest. This was to serve as a first step in building our models that were interpretable in the preferential sense, where we could easily compare the constant mean of the field across various models. However, in reality if these models are to be applied across various real world data sets, then nonconstant mean trends need to be considered. In this section we investigate the first extension of this using a linear trend, but this could be extended in future research to polynomial or spline-based surfaces and possible nonstationarity in the covariance structure. 


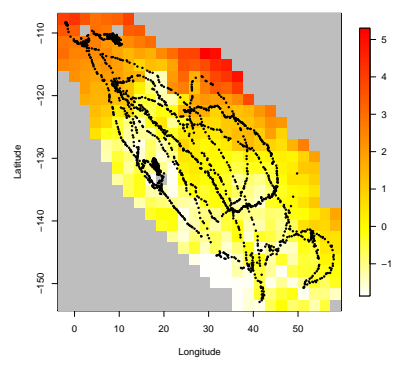

(a) SODA3 Temperature

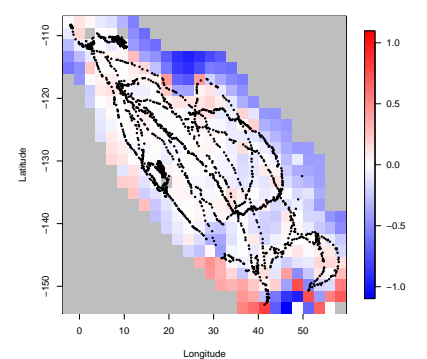

(d) $\operatorname{RMSPE}_{i}^{\text {Diff }}$

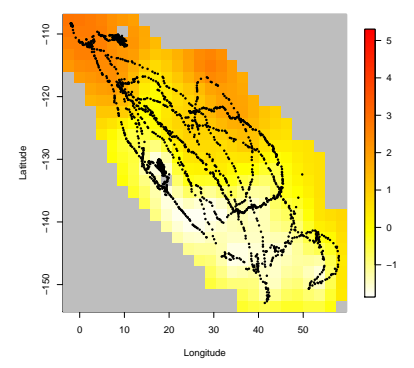

(b) Preferential Prediction

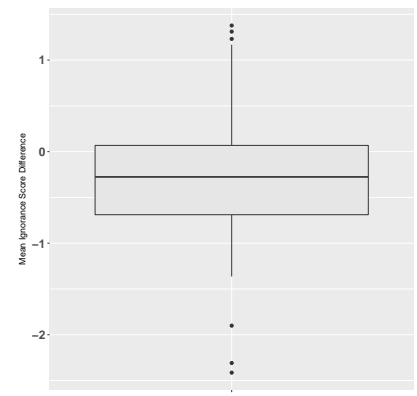

(e) $\mathrm{MIGN}_{j}^{\text {Diff }}$

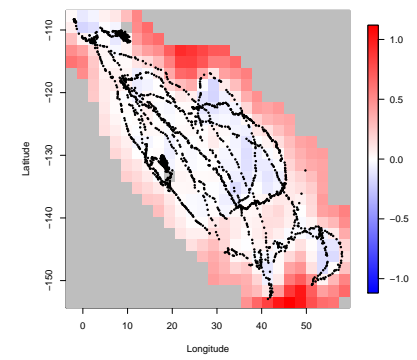

(c) Median of Prediction Difference

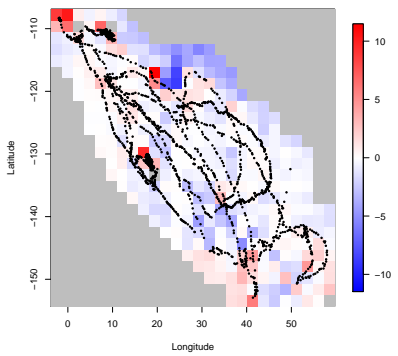

(f) $\operatorname{LIGN}_{i}^{\text {Diff }}$

Figure 9.3: Panel (a): Simple Ocean Data Assimilation ocean/sea ice version 3 (SODA3) reanalysis (Carton et al., 2018) model output $\left({ }^{\circ} \mathrm{C}\right)$. Panel (b): The mean preferential model prediction $\left({ }^{\circ} \mathrm{C}\right)$. Panel (c): Median of prediction difference between preferential and non-preferential models $\left({ }^{\circ} \mathrm{C}\right)$. Positive values imply that the preferential model tends to increases temperature prediction at that location and negative the opposite. Panels (d)-(f): Comparison of root-mean-square prediction error (RMSPE) difference, mean ignorance score (MIGN) difference and location ignorance score (LIGN) difference respectively across 50 simulated data sets. 
In the analysis of ocean temperature data during Sections 8.5 and 9.1 there was a noticeable increase in ocean temperature the further North in the data region the seals go. This was apparent in both the SST data shown Figure 8.11 and the temperatures at a depth of 50 meters in Figure 9.1. For example, a more realistic mean function for the SST analysis in Section 8.5 might be

$$
\mu(\mathbf{X})=\mu_{1}+\mu_{2} X_{1}+\mu_{3} X_{2}
$$

where $\mathbf{X}=\left(X_{1}, X_{2}\right)$ is the current location. This enables a mean trend in the form of a linear surface across the domain, which in the SST example might work better than a constant trend, as there appears to be an steady increase in SST towards the North-East.

A linear trend in the standard model that conditions on the sampling locations is simple to implement. The definition of the standard likelihood (marginal of $\mathbf{Y}$ ) in (2.5) would use

$$
\begin{aligned}
\mu(\mathbf{X}) & =\mathbf{D} \boldsymbol{\zeta} \\
& =\left(\begin{array}{cc}
1 & \mathbf{X}_{1} \\
\vdots & \vdots \\
1 & \mathbf{X}_{n}
\end{array}\right)\left(\begin{array}{l}
\mu_{1} \\
\mu_{2} \\
\mu_{3}
\end{array}\right), \\
& =\left(\begin{array}{ccc}
1 & X_{1,1} & X_{2,1} \\
\vdots & \vdots & \vdots \\
1 & X_{1, n} & X_{2, n}
\end{array}\right)\left(\begin{array}{l}
\mu_{1} \\
\mu_{2} \\
\mu_{3}
\end{array}\right), \\
& =\left(\begin{array}{c}
\mu_{1}+\mu_{2} X_{1,1}+\mu_{3} X_{2,1} \\
\vdots \\
\mu_{1}+\mu_{2} X_{1, n}+\mu_{3} X_{2, n}
\end{array}\right),
\end{aligned}
$$

where $\mathbf{X}_{j}=\left(X_{1, j}, X_{2, j}\right)$ is the $j$ th observation for $j=1, \ldots, n$. Also, this mean function can easily be substituted into the minimum mean square estimator from (2.14) and used in universal kriging to predict with a spatially varying trend.

Implementation of this mean trend into the full (preferential) likelihood requires a re-specification of $[\mathbf{S}]$ in the negative joint log-likelihood function. Again, this is relatively straightforward, since this does not impact on the SPDE specification of the covariance structure. TMB uses the SPDE approach to obtain a GMRF approximation to a Gaussian field density function with 0 mean. Therefore to evaluate our Gaussian field $\mathbf{S}$, we must specify the mean surface $\mu(\mathbf{X})$ and evaluate the GMRF approximation with respect 
to $\mathbf{S}-\mu(\mathbf{X})$ in each likelihood evaluation. As a consequence of this, theoretically any mean surface, be it splines or polynomials could be constructed in TMB and applied to the preferential likelihood. The biggest issue in these cases would be the increased number of parameters in a model which is already computationally challenging.

We assess how models with a mean trend from (9.1) perform with a reanalysis of the SST data from Section 8.5. In Section 9.2.1 we compare the preferential model performance with a constant trend compared to the preferential model with linear trend from (9.1). Next we compare preferential and non-preferential performance in Section 9.2 .2 with both models using the linear trend function from (9.1). Here we are fitting the exact same models as in Section 8.5 but replacing the mean trend.

\subsubsection{Comparison Between Constant and Linear Trend Preferential Performance}

In Figure 9.4 we compare predictions from the preferential model with a constant trend (results from Section 8.5) and the preferential model with a linear trend from (9.1), against the temperature data at a depth of 5 meters from the model outputs using the Simple Ocean Data Assimilation ocean/sea ice version 3 (SODA3) reanalysis (Carton et al., 2018). Both the preferential prediction plots (Panels (b)-(c)) show the mean prediction of the field over the same 50 data sets which each consist of 30 observations from the 13 tracks from the full data set.

At first sight there appears to be little difference between the two prediction models. Clearly neither preferential model fully captured the warmer areas of the temperature field along the Northern edges of the domain, although the southern areas are more comparable. To visualise the difference between the two models we compare the median of each coordinate of the vector $\mathbf{D}=\left(D_{1}, \ldots, D_{N}\right)$ for each of the $N=505$ prediction locations. Here we define,

$$
D_{i}=\left(\hat{S}_{1, i}^{P L}-\hat{S}_{1, i}^{P C}, \ldots, \hat{S}_{50, i}^{P L}-\hat{S}_{50, i}^{P C}\right)^{T},
$$

where $\hat{S}_{j, i}^{P L}$ is the preferential prediction at location $i$ in simulation $j$ using the preferential model with linear trend from $(9.1)$ and $\hat{S}_{j, i}^{P C}$ the equivalent preferential model with constant trend. We plot this in Panel (d) of Figure 9.4. It appears the linear trend model increased the median prediction in the North-East regions slightly, but more evident is reduced median prediction in the lower temperature regions towards the South in comparison to the constant trend. This makes sense if we observe the estimates $\hat{\mu}_{2}$ and 
$\hat{\mu}_{3}$ from (9.1) in Panel (e) of Figure 9.4, which shows that the mean trend was predicted to increase in the North-Easterly direction.

We assess the prediction accuracy in Figure 9.5 by plotting

$$
\begin{aligned}
\operatorname{RMSPE}_{i}^{\text {Diff }} & =\mathrm{RMSPE}_{i}^{\mathrm{PL}}-\mathrm{RMSPE}_{i}^{\mathrm{PC}}, \\
\mathrm{MIGN}_{j}^{\text {Diff }} & =\mathrm{MIGN}_{j}^{\mathrm{PL}}-\mathrm{MIGN}_{j}^{\mathrm{PC}}, \\
\mathrm{LIGN}_{i}^{\text {Diff }} & =\mathrm{LIGN}_{i}^{\mathrm{PL}}-\mathrm{LIGN}_{i}^{\mathrm{PC}},
\end{aligned}
$$

where PL and PC indicate the values of the scoring functions for the preferential models with linear and constant trend, respectively. The RMSPE comparison shows that the linear trend model improved point prediction across some locations, particularly in the South, but increased RMSPE in other areas such as the North-West. There was however a clear improvement in MIGN and LIGN overall when using the linear trend model, with no positive values of MIGN $_{j}^{\text {Diff }}$ recorded for any of the 50 data sets. This improvement in prediction using the preferential model with a linear trend shows the value in mean function specification, even when accounting for preferential movement.

To make sure there was no drawback in terms of prediction variance, which seems unlikely due to the ignorance scores, we compare the median of each coordinate of the vector $\mathbf{V}=\left(V_{1}, \ldots, V_{N}\right)$ such that

$$
V_{i}=\left(\hat{V}_{1, i}^{P L}-\hat{V}_{1, i}^{P C}, \ldots, \hat{V}_{50, i}^{P L}-\hat{V}_{50, i}^{P C}\right)^{T}
$$

where $\hat{V}_{j, i}^{P L}$ is the preferential prediction variance at location $i$ in simulation

$j$ using the preferential model with linear trend from (9.1) and $\hat{V}_{j, i}^{P C}$ the equivalent preferential prediction variance with constant trend. We plot the results in Panel (d) in Figure 9.5. In this case the central areas of high sampling tend to have a larger prediction variance when using the linear trend model, but the difference is fairly small. More extreme differences were evident in the outlying predictive regions where the linear trend model had a smaller median variance at most locations.

\subsubsection{Comparison Between Preferential and Non-Preferential Trend Performance}

Next we compare the performance of the preferential linear trend model with the non-preferential linear trend model in Figure 9.6. In Panel (a) the positive median prediction difference implies that the preferential model had a higher median prediction than the non-preferential equivalent. It 


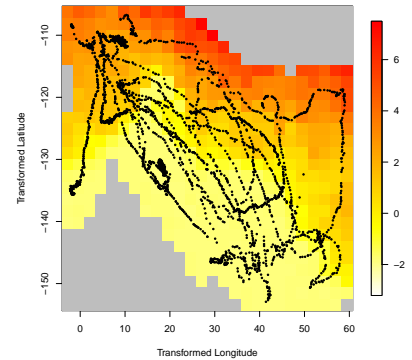

(a) SODA3 Temperature

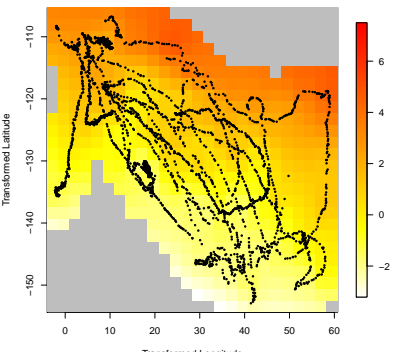

(c) Linear Trend Prediction

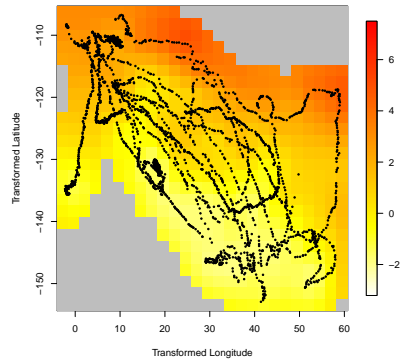

(b) Constant Trend Prediction

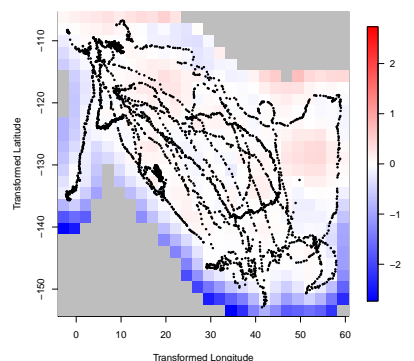

(d) Median of Prediction Difference

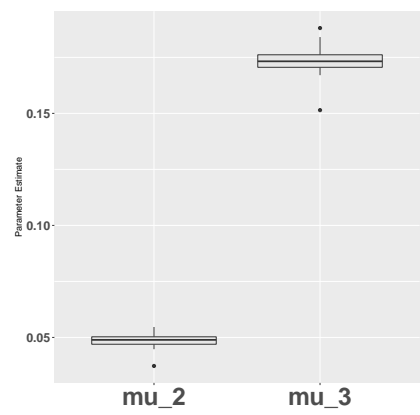

(e) Estimates of $\mu_{2}$ and $\mu_{3}$

Figure 9.4: Panels (a)-(c) Comparison between SODA3 data set model output with average preferential predictions (linear and constant mean trend) over 50 subsamples of the seas surface temperature data set described in Section $8.5\left({ }^{\circ} \mathrm{C}\right)$. Panel $(\mathrm{d})$ : The median difference between the two preferential models $\left({ }^{\circ} \mathrm{C}\right)$ with negative values of this difference meaning lower predicted values using the linear trend model and positive values greater predictions using this model in comparison to the constant trend equivalent. Panel (e): Comparison between $\hat{\mu}_{2}$ and $\hat{\mu}_{3}$ from (9.1). 


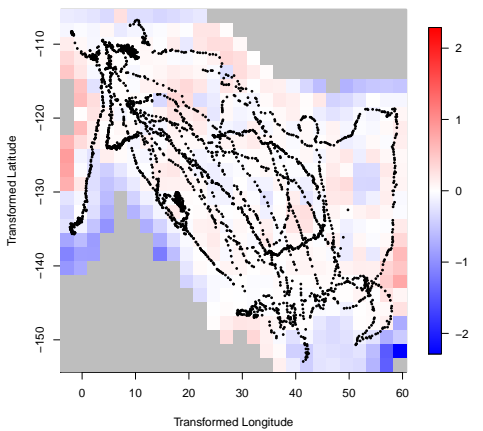

(a) $\operatorname{RMSPE}_{i}^{\text {Diff }}$

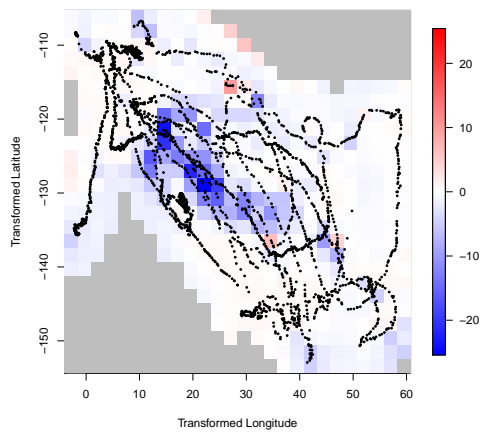

(c) $\operatorname{LIGN}_{j}^{\text {Diff }}$

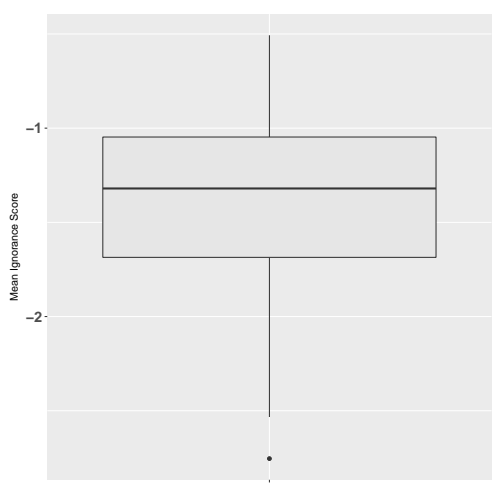

(b) $\operatorname{MIGN}_{i}^{\text {Diff }}$

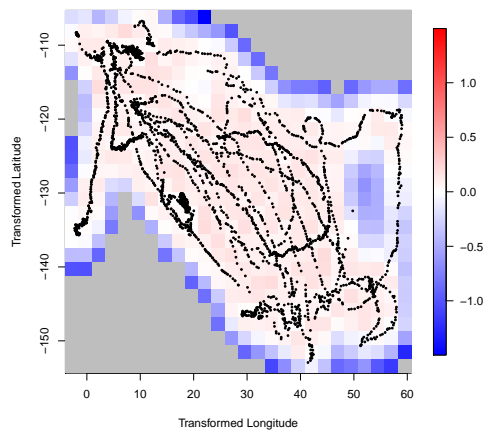

(d) Variance Comparison

Figure 9.5: Comparison of predictive performance compared to SODA3 model output between preferential models with constant and linear trends using scoring functions from $(9.3)$. 
appears that the linear trend models follow a somewhat similar pattern to the constant mean models which can be observed in Panel (b) of Figure 8.14. This pattern is that the preferential model predicts larger values of the temperature field in general, except for the central region.

In Panels (b)-(d) we compare the scoring functions

$$
\begin{aligned}
\mathrm{RMSPE}_{i}^{\text {Diff }} & =\mathrm{RMSPE}_{i}^{\mathrm{PL}}-\mathrm{RMSPE}_{i}^{\mathrm{NPL}}, \\
\mathrm{MIGN}_{j}^{\text {Diff }} & =\mathrm{MIGN}_{j}^{\mathrm{PL}}-\mathrm{MIGN}_{j}^{\mathrm{NPL}}, \\
\mathrm{LIGN}_{i}^{\text {Diff }} & =\mathrm{LIGN}_{i}^{\mathrm{PL}}-\mathrm{LIGN}_{i}^{\mathrm{NPL}},
\end{aligned}
$$

where PL and NPL indicate the values of the scoring functions for the preferential and non-preferential models with a linear trend, respectively. Again the patterns are similar to the scoring function comparison in the constant trend analysis, which can be seen in Figure 8.15. It appears that the preferential model performed better in general, with regularly smaller MIGN, LIGN and RMSPE values. Interestingly, if we compare the difference in ignorance scores here with the preferential model with linear trend from Section 9.2.1, it appears that the non-preferential linear trend model would outperform the preferential model with constant trend, further highlighting the importance of including a realistic mean structure in the model specification.

\subsubsection{Concluding Thoughts}

Including non-constant mean trends in preferential sampling is a natural extension of the methodology presented in this thesis and the preliminary results in this section show the impact that a more realistic trend might have in terms of spatial prediction. Although we specify a fairly simple trend function in (9.1), the implementation with TMB was very simple and made little difference computationally. As mentioned previously, further developments in this area could consider non-linear trend surfaces but also spatial trends which depend on spatially referenced covariates. Diggle and Ribeiro (2007) believe models of this type are more interesting, since instead of trying to simply describe the spatial trend, these models aim to explain this trend. In the case of marine mammal data, this could include other measures such as salinity, bathymetry and prey density. However one must be careful in over-specifying the mean trend surfaces. The spatial stochasticity is best captured through the modelling of the field covariance as to not provide misleading predictions and/or understanding of the latent process. Diggle and Ribeiro (2007) suggest that anything beyond quadratic surfaces should be avoided if possible. 


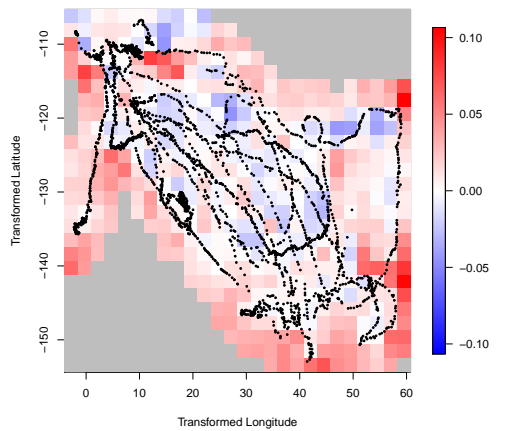

(a) Median of Prediction Difference

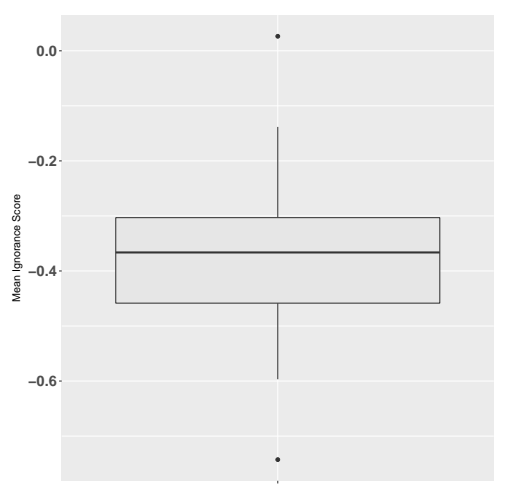

(c) $\mathrm{MIGN}_{j}^{\text {Diff }}$

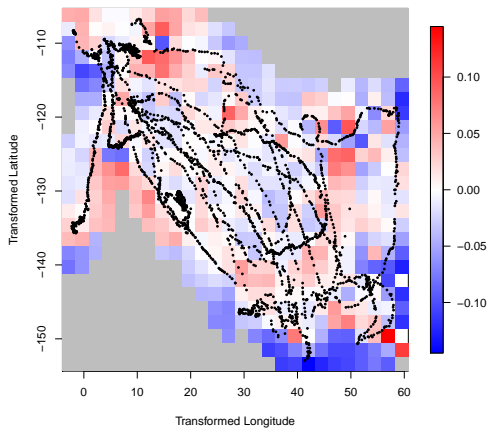

(b) $\mathrm{RMSPE}_{i}^{\mathrm{Diff}}$

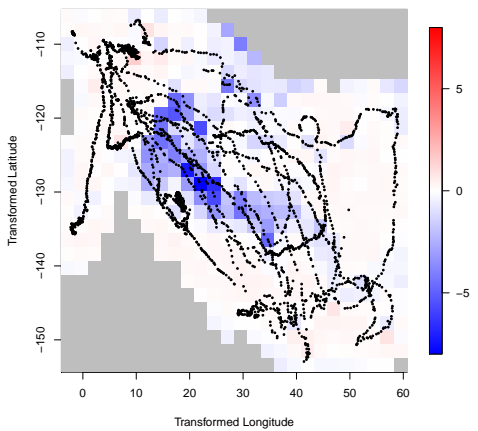

(d) $\operatorname{LIGN}_{i}^{\text {Diff }}$

Figure 9.6: Comparison of predictive performance compared to SODA3 model output between preferential and non-preferential models with linear trends using scoring functions from (9.5) 


\section{Chapter 10}

\section{Concluding Remarks and Future Work}

In this thesis we revised and improved previously proposed methods for preferential sampling in geostatistics and then used these to extend preferential sampling methodology to sampling systems that move through a domain of interest, such as ocean temperature bio-logging by marine mammals. The final aim was to provide groundwork for preferential sampling methods in ecology, through integration of marine mammal movement models within the preferential sampling framework.

To achieve this goal we had to first obtain a computationally viable and flexible approach to fitting preferential sampling models. This led us to the pioneering work of Diggle et al. (2010), who proposed MC methods to approximate the intractable likelihood that arises under preferential sampling, which we outlined in Chapter 4. However, working with this system exposed flaws within the derivation of the likelihood function. We proposed alternative MC solutions but noted that one should not ignore the effects of preferential sampling in the predictive distribution of the preferentially sampled latent field. We eventually decided to use a Laplace approximation to the preferential likelihood function, using the $R$ package Template Model Builder (TMB). This approach enabled us to more efficiently maximise the correct likelihood function, as well as providing flexibility in model specification not matched by other alternatives such as the R-INLA package. We assessed the performance of Laplace approximations for the preferential likelihood function and the resulting potential for correcting parameter estimates of a Matérn field with constant mean trend. Performance was clearly superior to that of the MC alternative in simulation studies and provided slightly different results in the Galicia example data set to that of Diggle et al. (2010).

The Laplace approximation method also provides a means to predict through the mode of the predictive distribution, which itself has an intractable form under preferential sampling. This allowed us to not only correct parameter estimates but also improve spatial prediction by using 
information provided by the relationship between the sampling locations and latent field to inform the prediction model. In Chapter 5 we assessed the performance of Laplace approximations in terms of prediction through the mode of the posterior at the optimal parameter values, with corrections showing the improved performance over kriging methods. We also assessed the predictive performance on a real data set consisting of daily ozone measurements taken by stationary monitoring sites across the USA in Chapter 6. Results were comparable when the data set analysed was a random subset of the full data set, but TMB reduced bias considerably over kriging in preferentially sampled subsets.

This work provided a base from which to apply preferential sampling methodology to bio-logging of ocean temperatures by marine mammals. We first discussed various modelling approaches used for marine mammal movement in literature in Chapter 7, with the goal of adapting these to create our preferential correlated random walk (PCRW) movement model which we derived in Chapter 8 . This movement model enabled the latent field we are measuring to influence the movement process itself, thereby capturing instances of preferential sampling. We showed how this model could be adapted for various movement patterns, with the latent process only impacting movement speed in one case, but the latent process altering movement direction and speed in others. We then displayed how data generated using these movement models result in preferential bias in both parameter estimates and subsequent prediction if ignored. Implementation of these movement models into the Laplace approximation preferential framework was not challenging. The flexibility of having full control over the model specification through the negative joint log-likelihood when using TMB, meant that we could easily change the sampling location distributions to account for the movement process. Performance of our methods was also good, particularly when movement direction and velocity depend on the latent process. When the latent field only affects movement speed, the preferential model showed little in the way of correction in either parameter estimates or spatial prediction. We also applied our methodology to sea surface temperature data collected by Southern elephant seals in the Southern Indian ocean. Here our preferential model varied in comparison with kriging methods, with a reduction of temperature prediction in certain areas and an increase in others. Comparison with sea surface temperatures obtained via satellite imagery showed possible improvements using our model, but we are wary to read too much into the prediction comparison due to the lack of time dependency in our model, meaning comparison was of a monthly average temperature. 
Finally in Chapter 9 we investigated some preliminary extensions of our work which we deem to be obvious next steps in the improvement and application of our preferential model framework. We first showed the ease at which we can adapt our modelling from sea surface temperature to ocean temperature data at various depths, which will be of more use to mapping ocean temperatures at depths not observable by satellite. Here the methodology does not change, but the manner in which we determine the sampling locations underwater when we only know the location at the end of the dive is not extensively investigated. We suggest that methods such as the dead reckoning algorithm (Wilson and Wilson, 1988) might provide approximations to the underwater locations, but for now we assume they are the same as location when the mammal surfaces. We also show how our preferential model can easily incorporate mean trends, with a comparison between linear and constant mean trends from the sea surface temperature real data simulation. The improved performance in the case of a linear trend model suggests that further development in this area may be of great benefit to our preferential model framework.

\subsection{Future Work}

In this section we outline, in what we believe to be decreasing order of importance, future extensions of the work in this thesis.

\subsubsection{Including Location Uncertainty}

As highlighted in Section 7.2.1 it is possible to incorporate observational uncertainty into the model for location measurements, which is an important feature for our preferential methods to be applied to real world data, in particular for ARGOS measurements which may contain significant measurement error. In $(7.3)$ we gave an example of how this might be integrated into the model definition by considering the data to consist of observed locations $\mathbf{Z}=\left(\mathbf{Z}\left(t_{1}\right), \ldots, \mathbf{Z}\left(t_{n}\right)\right)$ which are the true locations $\mathbf{X}=\left(\mathbf{X}\left(t_{1}\right), \ldots, \mathbf{X}\left(t_{n}\right)\right)$ with some measurement error. The simplest example would be $\mathbf{Z}\left(t_{k}\right)=\mathbf{X}\left(t_{k}\right)+\boldsymbol{\epsilon}\left(t_{k+1}\right)$ for some error variable $\boldsymbol{\epsilon}$, for which Jonsen et al. (2005) and Albertsen et al. (2015) claim the $t$-distribution is most suitable for ARGOS data.

As discussed in Section 7.2.1, location error can be included in a model which utilises Kalman filtering, although this will require more computational effort than current methods. However, the flexibility of TMB again becomes useful, since it could be used to integrate out multiple latent variables 
by assuming a hierarchical state space framework. Details and background on this on this in the non-preferential case can be found in Section 7.2.1. Under preferential sampling, if we assume there is no behavioural state in the model, then one appropriate factorisation would be

$$
\begin{aligned}
{[\mathbf{Z}, \mathbf{Y}] } & =\iint[\mathbf{Z}, \mathbf{Y}, \mathbf{S}] \mathrm{d} \mathbf{S} \mathrm{d} \mathbf{X}, \\
& =\iint[\mathbf{Z} \mid \mathbf{X}, \mathbf{Y}, \mathbf{S}][\mathbf{X}, \mathbf{Y}, \mathbf{S}] \mathrm{d} \mathbf{S} \mathrm{d} \mathbf{X}, \\
& =\iint[\mathbf{Z} \mid \mathbf{X}, \mathbf{Y}, \mathbf{S}][\mathbf{Y} \mid \mathbf{X}, \mathbf{S}][\mathbf{X} \mid \mathbf{S}][\mathbf{S}] \mathrm{d} \mathbf{S} \mathrm{d} \mathbf{X} .
\end{aligned}
$$

In this case the only new distribution is that of $\mathbf{Z} \mid \mathbf{X}, \mathbf{Y}, \mathbf{S}$, which depends on the measurement error model. Gaussian errors are easily implemented in TMB but so are $t$-distributed errors, as is shown by Albertsen et al. (2015).

We could even build upon this further if we were to include a behavioural state vector $(\boldsymbol{\beta})$. Then we would then have 3 latent factors

$$
[\mathbf{Z}, \mathbf{Y}]=\iiint[\mathbf{Z} \mid \mathbf{X}, \mathbf{Y}, \mathbf{S}, \boldsymbol{\beta}][\mathbf{Y} \mid \mathbf{X}, \mathbf{S}, \boldsymbol{\beta}][\mathbf{X} \mid \mathbf{S}][\boldsymbol{\beta} \mid \mathbf{S}][\mathbf{S}] \mathrm{d} \mathbf{S} \mathrm{d} \mathbf{X} \mathrm{d} \boldsymbol{\beta}
$$

although by this point the model may be becoming overly convoluted.

\subsubsection{Combining with Deterministic Model Outputs}

In the ocean temperature analyses at depths from Section 9.1 we compared our predictive models with output from the Simple Ocean Data Assimilation ocean/sea ice reanalysis (SODA) (Carton and Giese, 2008). The SODA models utilise oceanographic data from the World Ocean Database (WOD) collected by ship-based CTD sensors, profiling floats, moored and drifting buoys amongst many others (Locarnini et al., 2013) in coupled atmospheric and oceanic climate models developed by the National Oceanic and Atmospheric Administration's (NOAA) Geophysical Fluid Dynamics Laboratory (GFDL) (Delworth et al., 2006). These models are highly complex physical systems and provide ocean temperature prediction on a tripolar grid at a scale of approximately 28 kilometers longitude in the Southern Indian ocean.

Bearing this in mind, comparison with a small database of seal based observations is not particularly relevant. We are not trying to suggest an alternative or improved method over these complex systems. Instead these animal based profiles can be used to supplement these large models and databases, with the caveat that preferential sampling might be present and bias the inputs. Marine mammal based observations can be particularly 
useful in that they are recorded on a finer scale than SODA analysis, both in terms of latitude/longitude but also in terms of depth and can therefore offer finer scale inference. Furthermore, as discussed in Section 8.2, large models such as SODA require huge numbers of inputs which are often unavailable in sparsely sampled areas (for example the Southern Indian ocean). Consequently animal based data may be of particular value for the complex systems in these regions.

As a result, developing data assimilation methods for preferential sampling models would be of great value. This could be approached in multiple ways. One could be to use output from systems such as SODA as inputs in the preferential models to provide more accurate analysis. However, this would involve data measured on different spatial scales, so would require further model development. An alternative is to use preferential model output within the complex mathematical systems used by organisations such as NOAA. This could be to add an extra data sources to collections such as the World Ocean Database, where the marine mammal data has been preprocessed to account for preferential sampling, or alternatively as raw data inputs where the preferential correction is in the physical system models themselves.

\subsubsection{Introducing Time Varying Latent Fields}

In this thesis we have been assuming that the latent fields we are measuring do not change over time. In other words we model the sampled values as $Y_{k}=S\left(\mathbf{X}\left(t_{k}\right)\right)+\epsilon_{k}$ where $S(\cdot)$ is only function of location. This assumption is more suitable in the point process analyses such as the ozone example using monitoring sites in Section 6.1. In these cases the the data is observed at many locations at the same time, which might be daily/hourly averages, hence we have a substantial amount of data for any particular time we wish to analyse.

This is not the case in marine mammal data. Data is collected sparsely and irregularly in time and typically never repeated at the same location. Our data consists of measurements taken at unique locations in space and time. Therefore, the data we are analysing may be of a field that has changed between the observations taking place and the modelling assumption that $S(\cdot)$ is not a function of time could be more problematic. This would be more of an issue in measuring latent fields such as temperatures, however applications to other latent fields such as bathymetry would not be affected. In Section 8.2.2 we discussed an alternative approach to use a model of the form $Y_{k}=S\left(\mathbf{X}\left(t_{k}\right), t_{k}\right)+\epsilon_{k}$ so that $S(\cdot)$ is a function of both location and 
time. Implementing such a model might not be trivial though. As mentioned previously, we have no repeated measurements in space, so assessing whether field changes are due to the sampling location or time variation is difficult. A possible solution is aggregation of the data in areas of the domain, which could be treated as repeated observations. However part of the uniqueness in animal based measurements is the fine scale analysis they provide which would be lost in this case.

Another option could be to use historical data of the random field we wish to model. This might include values of the field across particular days, weeks or years integrated into the mean trend function. In the case of ocean temperature fields, we could build upon the current model by combining both historic and seasonal trends. For example we could propose a model of the form

$$
\tilde{S}\left(\mathbf{X}\left(t_{k}\right), t_{k}\right)=\gamma_{\text {Hist }}\left(\mathbf{X}\left(t_{k}\right)\right)+\gamma_{\text {Seas }}\left(\mathbf{X}\left(t_{k}\right)\right)+S\left(\mathbf{X}\left(t_{k}\right)\right),
$$

where $\gamma_{\text {Hist }}(\cdot)$ contains historical average information and $\gamma_{\text {Seas }}(\cdot)$ incorporates seasonal trends. A drawback of this approach however, is that we are in danger of over-specifying the trend of $\tilde{S}$, an issue warned against by Diggle and Ribeiro (2007).

These ideas and the issues that surround them highlight the difficulty that introducing time varying latent fields may entail. However, with more and more ocean temperature data being collected, it might be easier to include trends and correlations across seasons and years to bring some form of time variance to the field. Our solution in this thesis is to simply analyse real data over short periods of time so that the time varying nature of the ocean temperature fields has the smallest impact possible. But being able to combine more data into the preferential models across multiple months and seasons would only improve preferential sampling analysis.

\subsubsection{Improving Computational Efficiency}

Finally the computational efficiency of our methods still has room to improve. The Laplace approximations used in this thesis are more efficient than the Monte Carlo methods, but maximisation of the likelihood function in the case of preferential movement models is still computationally expensive. This problem arises in two different ways. Firstly the memory usage of the movement models when using TMB can cause problems when running on smaller machines with less than 16GB of Random Access Memory. We believe that this is due to the reverse mode automatic differentiation used 
by TMB as described in Section 4.4.2. This process stores intermediate variables from the computational graphs such as those in Figure 4.1 which in the case of complex movement models may be extremely large.

In addition to memory problems, convergence for each movement model example from simulation 1 in Section 8.4 took approximately 700 seconds. These simulations used the PCRW where gradients of the latent process were calculated in TMB using finite differences, with the data set consisting of up to 300 observations. There are 3 main reasons for this inefficiency which we now discuss.

1. Firstly, the gradient calculation requires simulating $\mathbf{S}$ at 3 times the number of sampling locations in addition to each location used in the SPDE mesh. This was to simulate $\mathbf{S}$ in a way that enabled us to calculate the gradient of $\mathbf{S}$ at any sampled location $\mathbf{X}$. Investigation into models which do not require gradients was conducted in simulation 2 of Section 8.4 but there was little difference between these models and the standard, non-preferential equivalent. Therefore, work into how to more efficiently calculate gradients or change in the latent field would be of great help in reducing time taken for likelihood maximisation.

2. Secondly, for completeness we were assuming that the latent field had smoothness $\kappa=2$ in the Matérn covariance structure so that the field was differentiable. Even though we used the SPDE approach outlined in Appendix A.1 to more efficiently evaluate the multivariate normal density function in this case, increasing the smoothness still reduces the sparsity of the precision matrix by increasing the neighbourhood effect in the GMRF, hence slowing computation (Lindgren et al., 2011). Preliminary investigations into whether we can use $\kappa=1$ with the finite difference gradient method showed no issues, but theoretically we would be using gradients of non-differentiable fields and therefore computational errors may occur in certain situations. Further study into this area could be useful to provide an assessment of the importance of smoothness parameter in these models. Oftentimes approaches using packages such as R-INLA assume $\kappa=1$ for computational and not theoretical reasons, which might be a possible approach in our case.

3. The final issue is regarding the SPDE mesh used in our TMB . cpp files, which we give a brief background on in Appendix A.2. For the simulations in this these we assessed prediction on a lattice grid. This was purely for ease of prediction assessment, but meant we needed to obtain predictions from TMB at hundreds of locations. To do this 
we specified the prediction locations as vertices in the meshes, therefore creating dense meshes which increased the time taken to evaluate the likelihood in each instance, but providing accurate prediction across the domain. Alternatively one could consider a multitude of other mesh systems, for example including restrictions on the length of edges, angles between edges and others. Again these are discussed in Appendix A.2 but more details can be found in Krainski et al. (2016). Investigatory simulations into non-lattice based meshes reduced the convergence time in simulation 1 of Section 8.4 from approximately 700 seconds to 335 seconds with little change in the parameter estimates. The issue in this case would be prediction at non-mesh locations, something which is discussed by Krainski et al. (2016) in R-INLA but would need to be applied to TMB. 


\section{Bibliography}

Adler, R.

1981. The Geometry of Random Fields, Classics in Applied Mathematics, 1st edition. Philadelphia, PA: Society for Industrial and Applied Mathematics (SIAM).

Albertsen, C. M., K. Whoriskey, D. Yurkowski, A. Nielsen, and J. M. Flemming

2015. Fast fitting of non-gaussian state-space models to animal movement data via template model builder. Ecology, 96(10):2598-2604.

AQS

2018. Air Quality System Data Mart [Accessed April 19, 2018 at https://www.epa.gov/outdoor-air-quality-data/download-daily-data]. US Environmental Protection Agency.

Auger-Méthé, M., C. M. Albertsen, I. D. Jonsen, A. E. Derocher, D. C. Lidgard, K. R. Studholme, W. D. Bowen, G. T. Crossin, and J. M. Flemming

2017. Spatiotemporal modelling of marine movement data using template model builder (TMB). Marine Ecology Progress Series, 565:237-249.

Auger-Méthé, M., C. Field, C. M. Albertsen, A. E. Derocher, M. A. Lewis, I. D. Jonsen, and J. M. Flemming

2016. State-space models' dirty little secrets: even simple linear gaussian models can have estimation problems. Scientific reports, 6:26677.

Baddeley, A., P. Gregori, J. Mateu, R. Stoica, and D. Stoyan

2006. Case studies in spatial point process modeling, Lecture Notes in Statistics, 1st edition. New York, NY: Springer.

Bakka, H., H. Rue, G.-A. Fuglstad, A. Riebler, D. Bolin, E. Krainski, D. Simpson, and F. Lindgren

2018. Spatial modelling with R-INLA: A review. arXiv preprint arXiv:1802.06350. 
Banerjee, S. and A. Gelfand 2003. On smoothness properties of spatial processes. Journal of Multivariate Analysis, 84(1):85 - 100 .

Banerjee, S., A. E. Gelfand, and C. F. Sirmans 2003. Directional rates of change under spatial process models. Journal of the American Statistical Association, 98(464):946-954.

Barthelmé, S., H. Trukenbrod, R. Engbert, and F. Wichmann 2013. Modeling fixation locations using spatial point processes. Journal of vision, 13(12):1-34.

Bell, B. M.

2005. CppAD: a package for $\mathrm{C}++$ algorithmic differentiation. http://www.coin-or.org/CppAD.

Benyahya, L., D. Caissie, A. St-Hilaire, T. B. Ouarda, and B. Bobée 2007. A review of statistical water temperature models. Canadian Water Resources Journal, 32(3):179-192.

Blackwell, P. 1997. Random diffusion models for animal movement. Ecological Modelling, 100(1-3):87-102.

Blangiardo, M. and M. Cameletti 2015. Spatial and spatio-temporal Bayesian models with $R$-INLA, 1st edition. New York, NY: John Wiley \& Sons.

Boehme, L., P. Lovell, M. Biuw, F. Roquet, J. Nicholson, S. E. Thorpe, M. P. Meredith, and M. Fedak 2009. Technical Note: Animal-borne CTD-Satellite Relay Data Loggers for real-time oceanographic data collection. Ocean Science, 5(4):685-695.

Bolker, B. M., B. Gardner, M. Maunder, C. W. Berg, M. Brooks, L. Comita, E. Crone, S. Cubaynes, T. Davies, P. Valpine, et al. 2013. Strategies for fitting nonlinear ecological models in R, AD Model Builder, and BUGS. Methods in Ecology and Evolution, 4(6):501-512.

Boyer, T. P., J. I. Antonov, O. K. Baranova, C. Coleman, H. E. Garcia, A. Grodsky, D. R. Johnson, R. A. Locarnini, A. V. Mishonov, T. D. O'Brien, et al.

2013. World ocean database. https://data.nodc.noaa.gov/woa/WOD13/DOC. 
Breed, G. A., D. P. Costa, I. D. Jonsen, P. W. Robinson, and J. MillsFlemming

2012. State-space methods for more completely capturing behavioral dynamics from animal tracks. Ecological Modelling, 235-236(0):49 - 58.

Breed, G. A., I. D. Jonsen, R. A. Myers, W. D. Bowen, and M. L. Leonard 2009. Sex-specific, seasonal foraging tactics of adult grey seals (halichoerus grypus) revealed by state-space analysis. Ecology, 90(11):3209-3221.

Brillinger, D. R.

2003. Simulating constrained animal motion using stochastic differential equations. Probability, statistics and their applications: papers in honor of Rabi Bhattacharya, 41:35-48.

Brillinger, D. R.

2012. Learning a Potential Function From a Trajectory, Pp. 361-364. New York, NY: Springer.

Brillinger, D. R., H. K. Preisler, A. A. Ager, J. G. Kie, and B. S. Stewart 2001. Modelling movements of free-ranging animals. Technical report, UCB Statistics Report 610.

Brillinger, D. R., H. K. Preisler, A. A. Ager, J. G. Kie, and B. S. Stewart 2002. Employing stochastic differential equations to model wildlife motion. Bulletin of the Brazilian Mathematical Society, 33(3):385-408.

Brillinger, D. R. and B. S. Stewart 1998. Elephant-seal movements: Modelling migration. Canadian Journal of Statistics, 26(3):431-443.

Brillinger, D. R., B. S. Stewart, and C. L. Littnan 2006. A meandering hylje. Festschrift for Tarmo Pukkila, Pp. 79-92.

Brillinger, D. R., B. S. Stewart, and C. L. Littnan 2008. Three months journeying of a Hawaiian monk seal, volume 2 of Collections, Pp. 246-264. Beachwood, OH: Institute of Mathematical Statistics.

Buckland, S., K. Newman, L. Thomas, and N. Koesters 2004. State-space models for the dynamics of wild animal populations. Ecological modelling, 171(1-2):157-175. 
Caissie, D., N. El-Jabi, and M. G. Satish 2001. Modelling of maximum daily water temperatures in a small stream using air temperatures. Journal of Hydrology, 251(1-2):14-28.

Cappé, O., E. Moulines, and T. Rydén 2005. Inference in Hidden Markov Models. New York, NY: SpringerVerlag.

Carton, J. A., G. A. Chepurin, L. Chen, and S. A. Grodsky 2018. Improved global net surface heat flux. Journal of Geophysical Research: Oceans, 123(5):3144-3163.

Carton, J. A. and B. S. Giese 2008. A reanalysis of ocean climate using Simple Ocean Data Assimilation (SODA). Monthly Weather Review, 136(8):2999-3017.

Chiles, J.-P. and P. Delfiner 2009. Geostatistics: modeling spatial uncertainty. Hoboken, NJ: John Wiley \& Sons.

Conn, P. B., J. T. Thorson, and D. S. Johnson 2017. Confronting preferential sampling when analysing population distributions: diagnosis and model-based triage. Methods in Ecology and Evolution, 8(11):1535-1546.

Craighead, Jr., F. C., J. J. Craighead, C. E. Cote, and H. K. Buechner 1972. Satellite and ground radiotracking of elk. NASA Special Publication, 262:99-111.

Cressie, N. 1990. The origins of kriging. Mathematical geology, 22(3):239-252.

da Silva Ferreira, G., D. Gamerman, et al. 2015. Optimal design in geostatistics under preferential sampling. Bayesian Analysis, 10(3):711-735.

Daigle, A., A. St-Hilaire, D. Peters, and D. Baird 2010. Multivariate modelling of water temperature in the Okanagan watershed. Canadian Water Resources Journal, 35(3):237-258.

Delworth, T. L., A. J. Broccoli, A. Rosati, R. J. Stouffer, V. Balaji, J. A. Beesley, W. F. Cooke, K. W. Dixon, J. Dunne, K. Dunne, et al. 2006. GFDL's CM2 global coupled climate models. Part I: Formulation and simulation characteristics. Journal of Climate, 19(5):643-674. 
Diggle, P. J., R. Menezes, and T.-l. Su 2010. Geostatistical inference under preferential sampling. Journal of the Royal Statistical Society: Series C (Applied Statistics), 59(2):191-232.

Diggle, P. J. and P. J. Ribeiro 2007. Model-based geostatistics. New York, NY: Springer Science \& Business Media.

Diggle, P. J., J. A. Tawn, and R. A. Moyeed 1998. Model-based geostatistics. Journal of the Royal Statistical Society. Series C (Applied Statistics), 47(3):pp. 299-350.

Dinsdale, D. and M. Salibian-Barrera 2018. Methods for preferential sampling in geostatistics. Journal of the Royal Statistical Society: Series C (Applied Statistics). In press.

Douglas, D. C., R. Weinzierl, S. C Davidson, R. Kays, M. Wikelski, and G. Bohrer 2012. Moderating Argos location errors in animal tracking data. Methods in Ecology and Evolution, 3(6):999-1007.

Dujon, A. M., R. T. Lindstrom, and G. C. Hays 2014. The accuracy of Fastloc-GPS locations and implications for animal tracking. Methods in Ecology and Evolution, 5(11):1162-1169.

Dunn, J. E. and P. S. Gipson 1977. Analysis of radio telemetry data in studies of home range. Biometrics, 33(1):85-101.

Evans, K., M.-A. Lea, and T. Patterson 2013. Recent advances in bio-logging science: technologies and methods for understanding animal behaviour and physiology and their environments. Deep Sea Research Part II: Topical Studies in Oceanography, $88: 1-6$.

Fancy, S. G., L. F. Pank, D. C. Douglas, C. H. Curby, and G. W. Garner 1988. Satellite telemetry: a new tool for wildlife research and management. Technical report, Fish and wildlife service Washington DC.

Fedak, M.

2004. Marine animals as platforms for oceanographic sampling: a situation for biology and operational oceanography. Memoirs of National Institute of Polar Research. Special issue, 58:133-147. 
Fedak, M.

2013. The impact of animal platforms on polar ocean observation. Deep Sea Research Part II: Topical Studies in Oceanography, 88-89:7-13. Fourth International Symposium on Bio-logging Science.

Fournier, D. A., H. J. Skaug, J. Ancheta, J. Ianelli, A. Magnusson, M. N. Maunder, A. Nielsen, and J. Sibert

2012. AD Model Builder: using automatic differentiation for statistical inference of highly parameterized complex nonlinear models. Optimization Methods and Software, 27(2):233-249.

Freitas, C., C. Lydersen, M. A. Fedak, and K. M. Kovacs

2008. A simple new algorithm to filter marine mammal Argos locations. Marine Mammal Science, 24(2):315-325.

Gelfand, A. E., P. Diggle, P. Guttorp, and M. Fuentes 2010. Handbook of spatial statistics. Boca Raton, FL: CRC press.

Gelfand, A. E., S. K. Sahu, and D. M. Holland

2012. On the effect of preferential sampling in spatial prediction. Environmetrics, 23(7):565-578.

Gelfand, A. E. and E. M. Schliep

2016. Spatial statistics and gaussian processes: A beautiful marriage. Spatial Statistics, 18:86-104.

Giles, M. B.

2013. Multilevel Monte Carlo methods. In Monte Carlo and Quasi-Monte Carlo Methods 2012, Pp. 83-103. Berlin: Springer.

Giles, M. B. 2015. Multilevel Monte Carlo methods. Acta Numerica, 24:259-328.

Glasserman, P.

2013. Monte Carlo methods in financial engineering, volume 53. New York, NY: Springer Science \& Business Media.

Gneiting, T.

2013. Strictly and non-strictly positive definite functions on spheres. Bernoulli, 19(4):1327-1349.

Gneiting, T. and A. E. Raftery

2007. Strictly proper scoring rules, prediction, and estimation. Journal of the American Statistical Association, 102(477):359-378. 
Góis, J., H. G. Pereira, and A. R. Salgueiro 2010. Geostatistics Applied to the City of Porto Urban Climatology, Pp. 65-75. Dordrecht: Springer Netherlands.

Goovaerts, P. 2008. Geostatistical Analysis of Health Data: State-of-the-Art and Perspectives, Pp. 3-22. Dordrecht: Springer Netherlands.

Griewank, A. and A. Walther

2008. Evaluating derivatives: principles and techniques of algorithmic differentiation. Philadelphia, PA: Society for Industrial and Applied Mathematics (SIAM).

Guennebaud, G. and B. Jacob 2010. Eigen v3. http://eigen.tuxfamily.org.

Guinet, C., J. Vacquié-Garcia, B. Picard, G. Bessigneul, Y. Lebras, A. C. Dragon, M. Viviant, J. P. Arnould, and F. Bailleul

2014. Southern elephant seal foraging success in relation to temperature and light conditions: insight into prey distribution. Marine Ecology Progress Series, 499:285-301.

Hallam, T. G. and S. A. Levin 1986. Mathematical ecology: an introduction, Biomathematics: Springer. Springer-Verlag.

Higdon, D.

1998. A process-convolution approach to modelling temperatures in the north atlantic ocean. Environmental and Ecological Statistics, 5(2):173190.

Högmander, H. and A. Särkkä 1999. Multitype spatial point patterns with hierarchical interactions. Biometrics, 55(4):1051-1058.

Jacobs, S. 2006. Observations of change in the southern ocean. Philosophical Transactions of the Royal Society of London A: Mathematical, Physical and Engineering Sciences, 364(1844):1657-1681.

Jeong, J. and M. Jun

2015. A class of Matérn-like covariance functions for smooth processes on a sphere. Spatial Statistics, 11:1-18. 
Johnson, D. S., J. M. London, M.-A. Lea, and J. W. Durban 2008. Continuous-time correlated random walk model for animal telemetry data. Ecology, 89(5):1208-1215.

Jonsen, I. D., J. M. Flemming, and R. A. Myers 2005. Robust state-space modeling of animal movement data. Ecology, 86(11):2874-2880.

Jørgensen, J. B. 2007. A critical discussion of the continuous-discrete extended Kalman filter. In Proceedings of the European Congress of Chemical Engineering.

Kalman, R. E.

1960. A new approach to linear filtering and prediction problems. Transactions of the ASME-Journal of Basic Engineering, 82(Series D):35-45.

Kent, J. T. et al.

1989. Continuity properties for random fields. The Annals of Probability, 17(4):1432-1440.

Krainski, E., F. Lindgren, D. Simpson, and H. Rue 2016. The R-INLA tutorial on SPDE models. http://www.rinla.org/examples/tutorials/spde-tutorial.

Krige, D. G.

1951. A statistical approach to some basic mine valuation problems on the Witwatersrand. Journal of the Southern African Institute of Mining and Metallurgy, 52(6):119-139.

Kristensen, K., A. Nielsen, C. W. Berg, H. Skaug, and B. M. Bell 2016. TMB: Automatic differentiation and Laplace approximation. Journal of Statistical Software, 70(5):1-21.

Kristensen, N. R., H. Madsen, and S. B. Jorgensen 2004. Parameter estimation in stochastic grey-box models. Automatica, 40(2):225-237.

Langrock, R., R. King, J. Matthiopoulos, L. Thomas, D. Fortin, and J. M. Morales

2012. Flexible and practical modeling of animal telemetry data: hidden Markov models and extensions. Ecology, 93(11):2336-2342. 
Lavancier, F., J. Møller, and E. Rubak 2015. Determinantal point process models and statistical inference. Journal of the Royal Statistical Society: Series B (Statistical Methodology), $77(4): 853-877$.

Levitus, S., J. I. Antonov, T. P. Boyer, and C. Stephens 2000. Warming of the world ocean. Science, 287(5461):2225-2229.

Lindgren, F. and H. Rue 2015. Bayesian spatial modelling with R-INLA. Journal of Statistical Software, 63(19):1-25.

Lindgren, F., H. Rue, and J. Lindström 2011. An explicit link between Gaussian fields and Gaussian Markov random fields: the stochastic partial differential equation approach. Journal of the Royal Statistical Society: Series B (Statistical Methodology), 73(4):423-498.

Liu, Y., B. C. Battaile, A. W. Trites, and J. V. Zidek 2015. Bias correction and uncertainty characterization of dead-reckoned paths of marine mammals. Animal Biotelemetry, 3(1):51-61.

Locarnini, R. A., A. V. Mishonov, J. I. Antonov, T. P. Boyer, H. E. Garcia, O. K. Baranova, M. M. Zweng, C. R. Paver, J. R. Reagan, D. R. Johnson, M. Hamilton, and D. Seidov

2013. World ocean atlas 2013, volume 1: Temperature. NOAA Atlas NESDIS, 73:1-40.

Lydersen, C., O. A. Nøst, P. Lovell, B. J. McConnell, T. Gammelsrød, C. Hunter, M. A. Fedak, and K. M. Kovacs

2002. Salinity and temperature structure of a freezing Arctic fjordmonitored by white whales (Delphinapterus leucas). Geophysical Research Letters, 29(23):2119-2122.

Mackintosh, N. A.

1952. The marking of whales. Nature, 169:435-437.

Matérn, B.

1960. Spatial Variation: Stochastic Models and Their Application to Some Problems in Forest Surveys and Other Sampling Investigations. Stockholm: Statens skogsforskningsinstitut. 
Matheron, G.

1963. Traité de géostatistique appliquée, number 2 in Mémoires du Bureau de Recherches Géologiques et Minières. Paris: Éditions Technip.

McIntyre, T., I. J. Ansorge, H. Bornemann, J. Plötz, C. A. Tosh, and M. N. Bester

2011. Elephant seal dive behaviour is influenced by ocean temperature: implications for climate change impacts on an ocean predator. Marine Ecology Progress Series, 441:257-272.

Metropolis, N., A. W. Rosenbluth, M. N. Rosenbluth, A. H. Teller, and E. Teller 1953. Equation of state calculations by fast computing machines. The journal of chemical physics, 21(6):1087-1092.

Minasny, B., J. A. Vrugt, and A. B. McBratney 2011. Confronting uncertainty in model-based geostatistics using Markov Chain Monte Carlo simulation. Geoderma, 163(3-4):150-162.

Mohanakumar, K. 2008. Stratosphere-Troposphere Exchange, Pp. 331-355. Dordrecht: Springer Netherlands.

Møller, J., A. R. Syversveen, and R. P. Waagepetersen 1998. Log Gaussian Cox Processes. Scandinavian Journal of Statistics, 25(3):451-482.

Møller, J. and R. P. Waagepetersen 2007. Modern statistics for spatial point processes. Scandinavian Journal of Statistics, 34(4):643-684.

Møller, J. K., H. Madsen, and J. Carstensen 2011. Parameter estimation in a simple stochastic differential equation for phytoplankton modelling. Ecological Modelling, 222(11):1793 - 1799.

Newman, K. B., S. T. Buckland, B. J. Morgan, R. King, D. L. Borchers, D. J. Cole, P. Besbeas, O. Gimenez, and L. Thomas 2014. Modelling population dynamics: model formulation, fitting and assessment using state-space methods. New York, NY: Springer.

Norris, K. S. and R. L. Gentry

1974. Capture and harnessing of young California gray whales, Eschrichtius robustus. Marine Fisheries Review, 36(4):58-64. 
Ozturk, D. and F. Kilic 2016. Geostatistical approach for spatial interpolation of meteorological data. Anais da Academia Brasileira de Ciências, 88(4):2121-2136.

Pati, D., B. J. Reich, and D. B. Dunson 2011. Bayesian geostatistical modelling with informative sampling locations. Biometrika, 98(1):35-48.

Patterson, T. A., A. Parton, R. Langrock, P. G. Blackwell, L. Thomas, and R. King 2017. Statistical modelling of individual animal movement: an overview of key methods and a discussion of practical challenges. AStA Advances in Statistical Analysis, 101(4):399-438.

Patterson, T. A., L. Thomas, C. Wilcox, O. Ovaskainen, and J. Matthiopoulos

2008. State-space models of individual animal movement. Trends in ecology \& evolution, 23(2):87-94.

Pebesma, E., D. Cornford, G. Dubois, G. B. Heuvelink, D. Hristopulos, J. Pilz, U. Stöhlker, G. Morin, and J. O. Skøien

2011. INTAMAP: the design and implementation of an interoperable automated interpolation web service. Computers $\&$ Geosciences, 37(3):343352 .

Preisler, H. K., A. A. Ager, and M. J. Wisdom

2013. Analyzing animal movement patterns using potential functions. Ecosphere, 4(3):1-13.

R Core Team 2013. R: A Language and Environment for Statistical Computing. $\mathrm{R}$ Foundation for Statistical Computing, Vienna, Austria.

Rayner, G. W.

1939. Preliminary results of the marking of whales by the discovery committee. Nature, 144:999-1002.

Ribeiro, M. I.

2004. Kalman and extended kalman filters: Concept, derivation and properties. Technical report, Institute for Systems and Robotics, Lisbon.

Ripley, B. D.

2009. Stochastic simulation, volume 316. New York, NY: John Wiley \& Sons. 
Robert, C. P. and G. Casella

2004. Monte Carlo Statistical Methods. Springer, New York, NY.

Robusto, C. C.

1957. The cosine-haversine formula. The American Mathematical Monthly, 64(1):38-40.

Roemmich, D., S. Riser, R. Davis, and Y. Desaubies

2004. Autonomous profiling floats: Workhorse for broad-scale ocean observations. Marine Technology Society Journal, 38(2):21-29.

Roquet, F., J.-B. Charrassin, S. Marchand, L. Boehme, M. Fedak, G. Reverdin, and C. Guinet

2011. Delayed-mode calibration of hydrographic data obtained from animal-borne satellite relay data loggers. Journal of Atmospheric and Oceanic Technology, 28(6):787-801.

Roquet, F., C. Guinet, and M. Hindell

2014a. A Southern Indian Ocean hydrographic database obtained with instrumented elephant seals. British Oceanographic Data Centre, Natural Environment Research Council.

Roquet, F., G. Williams, M. A. Hindell, R. Harcourt, C. McMahon, C. Guinet, J.-B. Charrassin, G. Reverdin, L. Boehme, P. Lovell, et al. 2014b. A Southern Indian Ocean database of hydrographic profiles obtained with instrumented elephant seals. Scientific data, 1:140028.

Roquet, F., C. Wunsch, G. Forget, P. Heimbach, C. Guinet, G. Reverdin, J.-B. Charrassin, F. Bailleul, D. P. Costa, L. A. Huckstadt, et al.

2013. Estimates of the Southern Ocean general circulation improved by animal-borne instruments. Geophysical Research Letters, 40(23):61766180 .

Roulston, M. S. and L. A. Smith 2002. Evaluating probabilistic forecasts using information theory. Monthly Weather Review, 130(6):1653-1660.

Rubinstein, R. Y. and D. P. Kroese 2017. Simulation and the Monte Carlo method, volume 10 of Wiley Series in Probability and Statistics, 3rd edition. Hoboken, NJ: John Wiley \& Sons. 
Rue, H. and L. Held 2005. Gaussian Markov Random Fields: Theory and Applications. New York, NY: Chapman and Hall/CRC.

Rue, H., S. Martino, and N. Chopin 2009. Approximate Bayesian inference for latent Gaussian models by using integrated nested Laplace approximations. Journal of the Royal Statistical Society: Series B (statistical methodology), 71(2):319-392.

Russell, J. C., E. M. Hanks, M. Haran, and D. Hughes 2018. A spatially varying stochastic differential equation model for animal movement. Ann. Appl. Stat., 12(2):1312-1331.

Sampson, P. D. 2010. Constructions for nonstationary spatial processes. Handbook of spatial statistics, Pp. 119-130.

Sampson, P. D. and P. Guttorp 1992. Nonparametric estimation of nonstationary spatial covariance structure. Journal of the American Statistical Association, 87(417):108-119.

Scheffer, V. B., C. H. Fiscus, and E. I. Todd 1984. History of scientific study and management of the Alaskan fur seal, Callorhinus ursinus, 1786-1964. NOAA technical report NMFS SSRF, 780 .

Shaddick, G. and J. V. Zidek 2014. A case study in preferential sampling: Long term monitoring of air pollution in the UK. Spatial Statistics, 9:51-65.

Siegert, S., C. A. Ferro, and D. B. Stephenson 2014. Evaluating ensemble forecasts by the ignorance score-correcting the finite-ensemble bias. arXiv preprint arXiv:1410.8249.

Simpson, D., F. Lindgren, and H. Rue 2012. In order to make spatial statistics computationally feasible, we need to forget about the covariance function. Environmetrics, 23(1):65-74.

Skaug, H. J. and D. A. Fournier

2006. Automatic approximation of the marginal likelihood in nonGaussian hierarchical models. Computational Statistics $\mathcal{G}$ Data Analysis, 51(2):699 - 709 . 
Stewart, B. S., S. Leatherwood, P. K. Yochem, and M.-P. Heide-Jørgensen 1989. Harbor seal tracking and telemetry by satellite. Marine Mammal Science, 5(4):361-375.

Thomson, J., L. Börger, M. Christianen, N. Esteban, J.-O. Laloe, and G. Hays 2017. Implications of location accuracy and data volume for home range estimation and fine-scale movement analysis: comparing Argos and Fastloc-GPS tracking data. Marine Biology, 164(10):204.

Ungar, E. D., Z. Henkin, M. Gutman, A. Dolev, A. Genizi, and D. Ganskopp 2005. Inference of animal activity from GPS collar data on free-ranging cattle. Rangeland Ecology \& Management, 58(3):256-266.

Votier, S. C., S. Bearhop, M. J. Witt, R. Inger, D. Thompson, and J. Newton

2010. Individual responses of seabirds to commercial fisheries revealed using GPS tracking, stable isotopes and vessel monitoring systems. Journal of Applied Ecology, 47(2):487-497.

Weimerskirch, H., F. Bonadonna, F. Bailleul, G. Mabille, G. Dell'Omo, and H.-P. Lipp

2002. GPS tracking of foraging albatrosses. Science, 295(5558):1259-1259.

Whoriskey, K., M. Auger-Méthé, C. M. Albertsen, F. G. Whoriskey, T. R. Binder, C. C. Krueger, and J. Mills Flemming

2017. A hidden Markov movement model for rapidly identifying behavioral states from animal tracks. Ecology and evolution, 7(7):2112-2121.

Wilson, R. and M.-P. Wilson 1988. Dead reckoning: a new technique for determining penguim movements at sea. Meeresforschung, 32(2):155-158.

Yang, H., G. Chen, Q. Tang, and P. Hess 2016. Quantifying isentropic stratosphere-troposphere exchange of ozone. Journal of Geophysical Research: Atmospheres, 121(7):3372-3387.

Zidek, J. V., G. Shaddick, and C. G. Taylor 2014. Reducing estimation bias in adaptively changing monitoring networks with preferential site selection. Ann. Appl. Stat., 8(3):1640-1670. 


\section{Appendix A}

\section{Efficient Evaluation of High Dimensional Gaussian Fields}

One of the computational limitations of using the preferential likelihood functions described in Section 4 is the requirement of simulating high dimensional Gaussian fields (GFs). Typically, the computational cost of these multivariate normal distributions is $\mathcal{O}\left(N^{3}\right)$ due to the factorising of dense $N \times N$ covariance matrices. In the case of MC approximations described in Section 4.3 , we would require evaluating a new $G F[\mathbf{S} \mid \mathbf{Y}, \mathbf{X}]$ for each $\mathrm{MC}$ simulation, whilst in the Laplace approximation we also require $[\mathbf{S}]$. Lindgren et al. (2011) summarise many of the attempts at solving "the big $N$ problem" with one of the most popular being the the Gaussian Markov random field (GMRF) representation discussed in Rue et al. (2009). A GMRF is simply a Gaussian random field $\mathbf{S}=\left(S_{1}, \ldots S_{N}\right) \sim N_{N}(\mathbf{D} \boldsymbol{\zeta}, \Sigma)$ such that

$$
\left[S_{i} \mid \mathbf{S}_{-i}\right]=\left[S_{i} \mid \mathbf{S}_{j}: j \in \delta_{i}\right]
$$

for some set of neighbours $\delta_{i}$ for each location $i$. In this case the precision matrix $\mathbf{Q}=\Sigma^{-1}$ has the property that $\mathbf{Q}_{i, j}=0 \Longleftrightarrow j \notin \delta_{i} \cup\{i\}$ (Rue and Held, 2005). Therefore GMRF's are discretely indexed representations of GF's that utilise Markov properties to decrease the number of non-zero entries in an $N \times N$ covariance matrix, typically reducing the computation cost to $\mathcal{O}\left(N^{3 / 2}\right)$ in 2-dimensions.

\section{A.1 Stochastic Partial Differential Equation Approximation}

Methods that utilise GMRF representations of GFs require constructing a sparse precision matrix $\mathbf{Q}$ that closely represents the covariance of the GF. We use an approach by Lindgren et al. (2011) who show how GMRF representations of GFs with Matérn covariance (as defined in (2.4)), can be constructed through the solution to a stochastic partial differential equation (SPDE) when driven by white noise. 
Lindgren and Rue (2015); Lindgren et al. (2011) show that a GF $S$ with Matérn covariance is a solution to the SPDE

$$
\left(\phi^{-2}-\Delta\right)^{\alpha / 2}(\tau S(\mathbf{x}))=\mathcal{W}(\mathbf{x}), \quad \mathbf{x} \in \mathcal{D},
$$

where $\tau$ controls the variance, $\alpha$ the smoothness, $\phi$ is the scale parameter from the Matérn function in $(2.4), \mathcal{W}$ is a spatial white noise process and $\mathcal{D} \subset \mathbb{R}^{2}$ is the spatial domain. The Laplacian operator $\Delta$ is defined in our 2-dimensional case as

$$
\Delta=\frac{\partial^{2}}{\partial x_{1}^{2}}+\frac{\partial^{2}}{\partial x_{2}^{2}}
$$

where $\mathbf{x}=\left(x_{1}, x_{2}\right)$.

The Matérn function in (2.4) and the SPDE in (A.1) are coupled via $\alpha=\kappa+d / 2$ where $d$ is the dimension of the process, and the marginal variance

$$
\sigma^{2}=\frac{\Gamma(\kappa)}{\Gamma(\alpha)(4 \pi)^{d / 2} \phi^{-2 \kappa} \tau^{2}}
$$

For irregular point data Lindgren et al. (2011) propose a GMRF representation of (A.1) be constructed using a triangulation of the domain. First we observe that for a set of test functions $\boldsymbol{\xi}=\left\{\xi_{k}: k=1, \ldots m\right\}$ the stochastic weak solution of the SPDE in (A.1) is any random field $S$ such that

$$
\int_{\mathcal{D}} \xi_{k}(\mathbf{x})\left(\phi^{-2}-\Delta\right)^{\alpha / 2}(\tau S(\mathbf{x})) \mathrm{d} \mathbf{x}=\int_{\mathcal{D}} \xi_{k}(\mathbf{x}) \mathrm{d} \mathcal{W}(\mathbf{x}) .
$$

Next we construct finite element solution to the SPDE in (A.1) as

$$
\tau S(\mathbf{x})=\sum_{k=1}^{m} \psi_{k}(\mathbf{x}) w_{k},
$$

for some basis functions $\boldsymbol{\psi}=\left(\psi_{1}, \ldots, \psi_{m}\right)$ and Gaussian weights $\mathbf{w}=$ $\left(w_{1}, \ldots, w_{m}\right)$, where $m$ is the number of vertices in the triangulation. The Markov structure is implemented using piecewise linear basis functions such that $\psi_{k}$ equals 1 at vertex $k$ and 0 at all other vertices. Therefore values of the field at vertices are entirely determined by the weights $\mathbf{w}$ and any points in between calculated via linear interpolation from the 3 surrounding vertices. Hence, the joint distribution of $\mathbf{w}$ determines the distribution of the continuous solution.

The finite element solution in (A.3) can be obtained by finding the distribution of $\mathbf{w}$ from $(\mathrm{A} .3)$ that satisfies the condition in $(\mathrm{A} .2)$ for appropriate test functions $\xi_{k}$ 's. Lindgren et al. (2011) choose $\xi_{k}=\left(\phi^{-2}-\Delta\right)^{1 / 2} \psi_{k}$ for 
$\alpha=1$ and $\xi_{k}=\psi_{k}$ for $\alpha=2$ which are denoted the least squares and Galerkin solutions respectively (see Appendix C in Lindgren et al. (2011)). For $\alpha \geq 3$ on the LHS of (A.1) they set $\alpha=2$ and replace the RHS by a field generated using $\alpha-2$, then let $\xi_{k}=\psi_{k}$.

Using these test functions we can write the precision matrix $\mathbf{Q}_{\alpha, \phi}$ for the weights $\mathbf{w}$ as a combination of $m \times m$ matrices $\mathbf{C}, \mathbf{G}$ and $\mathbf{K}_{\phi}$,

$$
\begin{aligned}
\mathbf{C}_{i, j} & =\left\langle\psi_{i}, \psi_{j}\right\rangle_{\mathcal{D}}, \\
\mathbf{G}_{i, j} & =\left\langle\Delta \psi_{i}, \Delta \psi_{j}\right\rangle_{\mathcal{D}}, \\
\left(\mathbf{K}_{\phi}\right)_{i, j} & =\phi^{-2} \mathbf{C}_{i, j}+\mathbf{G}_{i, j},
\end{aligned}
$$

where

$$
\langle f, g\rangle_{\mathcal{D}}=\int_{\mathcal{D}} f(\mathbf{x}) g(\mathbf{x}) \mathrm{d} \mathbf{x} .
$$

This combination depends on the smoothness $\alpha$ but can be calculated recursively as

$$
\begin{aligned}
\mathbf{Q}_{1, \phi} & =\mathbf{K}_{\phi}, \\
\mathbf{Q}_{2, \phi} & =\mathbf{K}_{\phi} \mathbf{C}^{-1} \mathbf{K}_{\phi}, \\
\mathbf{Q}_{\alpha, \phi} & =\mathbf{K}_{\phi} \mathbf{C}^{-1} \mathbf{Q}_{\alpha-2, \phi} \mathbf{C}^{-1} \mathbf{K}_{\phi}, \quad \text { for } \quad \alpha=3,4, \ldots
\end{aligned}
$$

This result does not yet create Markov fields however, since $\mathbf{C}$ is nondiagonal and hence $\mathbf{C}^{-1}$ is not sparse. Therefore Lindgren et al. (2011) suggest replacing $\mathbf{C}$ with the diagonal matrix $\tilde{\mathbf{C}}_{i i}=\sum_{j} \mathbf{C}_{i j}=\left\langle\psi_{i}, 1\right\rangle_{\mathcal{D}}$, which then yields a GMRF model.

Hence we have a finite element solution $\mathbf{w} \sim N_{m}\left(0, \mathbf{Q}_{\alpha, \phi}^{-1}\right)$ to the SPDE in (A.1), which provides a mapping from the parameters of the Matérn GF to the elements of a GMRF precision matrix. Further details on the derivations in this section can be found in Lindgren and Rue (2015); Lindgren et al. (2011); Simpson et al. (2012).

\section{A.2 Implementation of SPDE's in TMB}

We adopt the SPDE approach to obtain a GMRF approximation for our Gaussian field $S$ via TMB's built-in compatibility with R-INLA (Integrated Nested Laplace Approximations) (Rue et al., 2009). The R-INLA package, available to download at www.r-inla.org, provides the user with a template to create spatial meshes for use in the finite approximation method described in Appendix A.1 through the function inla.mesh.create. These 
meshes are then used to calculate the sparse precision matrices required for the GMRF approximation which we wish to then use within our TMB model.

In Figure A.1 we give an example of a sampling locations in red and corresponding meshes for a variety of restrictions implemented via inputs into the function inla.mesh.create. These meshes are created using the Constrained Refined Delaunay Triangulation (CRDT) method with Neumann boundaries which inflate variances near the boundary. Therefore we extended these boundaries to move the boundary effects outside the domain of interest. Furthermore, in general we want small regularly shaped triangles for the smallest error (Bakka et al., 2018), however the number of triangles in the mesh also impacts on the computational performance of the approximation. The first mesh in Figure A.1 is a simple triangulation between the sampling locations, however in this case the mesh contains triangles with very inconsistent and small angles throughout the domain. To resolve this, the second plot introduces a minimum angle of $21^{\circ}$ for each triangle. The third plot adds more restrictions in restricting the length of each edge to create a more consistent triangulation across the domain, whilst the final plot imposes a $20 \times 20$ spatial grid to be vertices of the mesh in addition to a minimum angle of $21^{\circ}$.

The properties imposed on the meshes depend on the users goal. Sparse triangulation in regions of little data improve computational efficiency, but reduce the prediction accuracy. Likewise, dense triangulation in regions of high sampling intensity decrease computational efficiency but provide higher accuracy. The edges are not restricted to the sampling locations either - for example we could impose a restriction of the closeness of edges to reduce the formation of small triangles near clusters of sampling locations.

Generally throughout this thesis we will be using lattice based meshes such as the final plot in Figure A.1. This is done for two reasons. Firstly, in the case of point process models we are using an intensity of the form in (3.4) which requires approximation of $\int_{\mathcal{D}} \exp \{\beta \mathbf{S}(\mathbf{u})\}$ du. Hence, by including a lattice within our spatial region for which we know the area of each interior rectangle, it is easier to approximate this integral. We show this in Figure A.2. Here we use the approximation

$$
[\mathbf{X} \mid \mathbf{S}]=\prod_{i=1}^{n} \frac{\exp \left\{\beta \mathbf{S}\left(\mathbf{X}_{i}\right)\right\}}{\int_{\mathcal{D}} \exp \{\beta \mathbf{S}(\mathbf{u})\} \mathrm{d} \mathbf{u}}=\prod_{i=1}^{n} \frac{\exp \left\{\beta \mathbf{S}\left(\mathbf{X}_{i}\right)\right\}}{\sum_{j=1}^{N} A \exp \left\{\beta \mathbf{S}\left(\mathbf{u}_{j}\right)\right\}},
$$

where $A$ is the area of each lattice rectangle, the $\mathbf{X}_{i}$ 's are the red points and $\mathbf{u}_{j}$ 's the blue points. Note that $n=5$ is the number of sampled locations and $N=25$ is the number of lattice points. 


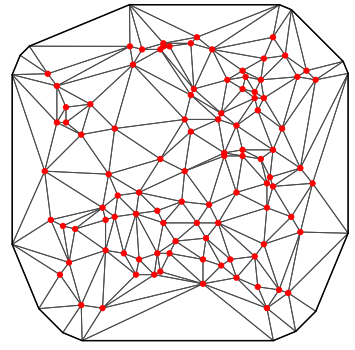

(a) No refinement

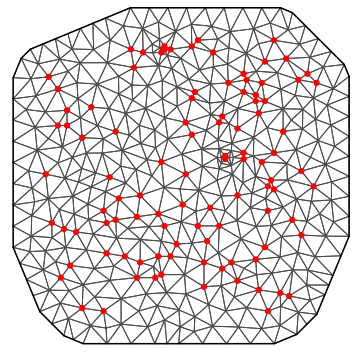

(c) Minimum Angle and Max Edge Length

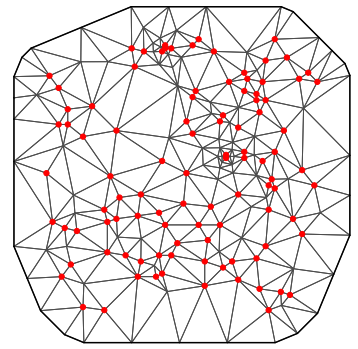

(b) Minimum Angle

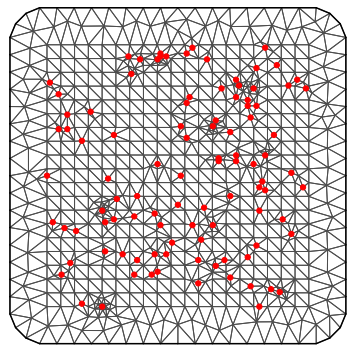

(d) Lattice, Minimum Angle

Figure A.1: Comparison of various spatial meshes using inla.mesh.create function in R-INLA. Each label is the type of refinement used in the specification of the mesh. 


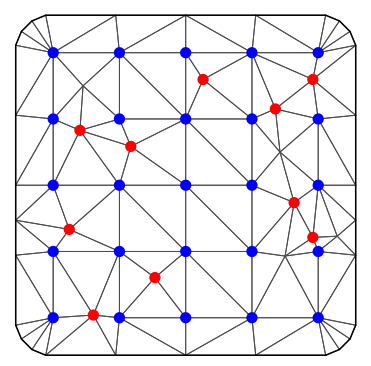

Figure A.2: Example of lattice and sampling location combined mesh. Here the red points are sampling locations and blue points the lattice points used to approximate the integral of the intensity function and for prediction comparison.

Secondly, we also use the mesh for prediction through the mode of $\mathbf{S} \mid \mathbf{X}, \mathbf{Y}$. In the examples where we repeatedly simulate new data sets across the same spatial domain, retaining the same lattice grid within each mesh enabled quick and easy comparison of predictions across the lattice, no matter the sampling locations of each data set. In the case of Figure A.2 the prediction locations would be the $\mathbf{u}_{j}$ 's (blue points) for $j=1, \ldots, N$.

Utilising R-INLA's built in functions to create a spatial mesh and corresponding components of the sparse precision matrix of a GMRF, can be used to more efficiently evaluate the large dimensional multivariate normal density required in (4.21) (Blangiardo and Cameletti, 2015). However before we can use this package, we must consider the fact that R-INLA only implements the SPDE approximation for $\alpha \in(0,2]$. This is fine in most applications, but at the largest smoothness of $\alpha=2(\kappa=1)$, we are assuming the Matérn field is mean-square continuous but not mean-square differentiable (see definitions from Section 2.2.3). In some cases this might not suit the problem at hand. For example, when derivatives of the fields are required, which is the case in our application to marine mammal based preferential sampling in Chapter 8.

Therefore, we want to extend the currently supported approximations in R-INLA to $\alpha=3(\kappa=2)$ and implement this in TMB via the built-in compatibility with R-INLA. To begin, note that for $\alpha=2$ we can expand 
the formula for $\mathbf{Q}_{2, \phi}$ from $(\mathrm{A} .4)$ as

$$
\begin{aligned}
& \mathbf{Q}_{2, \phi}=\mathbf{K}_{\phi} \mathbf{C}^{-1} \mathbf{K}_{\phi}, \\
& \mathbf{Q}_{2, \phi}=\left(\phi^{-2} \mathbf{C}+\mathbf{G}\right) \mathbf{C}^{-1}\left(\phi^{-2} \mathbf{C}+\mathbf{G}\right), \\
& \mathbf{Q}_{2, \phi}=\phi^{-4} \mathbf{C}+2 \phi^{-2} \mathbf{G}+\mathbf{G C}^{-1} \mathbf{G}, \\
& \mathbf{Q}_{2, \phi}=\phi^{-4} \mathbf{M}_{0}+2 \phi^{-2} \mathbf{M}_{1}+\mathbf{M}_{2},
\end{aligned}
$$

where $\mathbf{M}_{0}=\mathbf{C}, \mathbf{M}_{1}=\mathbf{G}$ and $\mathbf{M}_{2}=\mathbf{G C}^{-1} \mathbf{G}$. Hence, we can use the R-INLA function inla.spde2. matern for $\alpha=2$ to obtain $\mathbf{M}_{1}, \mathbf{M}_{2}$ and $\mathbf{M}_{3}$ before running the model, since these do not depend on $\phi$.

Now we expand $\mathbf{Q}_{3, \phi}$

$$
\begin{aligned}
& \mathbf{Q}_{3, \phi}=\mathbf{K}_{\phi} \mathbf{C}^{-1} \mathbf{Q}_{1, \phi} \mathbf{C}^{-1} \mathbf{K}_{\phi}, \\
& \mathbf{Q}_{3, \phi}=\mathbf{K}_{\phi} \mathbf{C}^{-1} \mathbf{K}_{\phi} \mathbf{C}^{-1} \mathbf{K}_{\phi}, \\
& \mathbf{Q}_{3, \phi}=\left(\phi^{-2} \mathbf{C}+\mathbf{G}\right) \mathbf{C}^{-1}\left(\phi^{-2} \mathbf{C}+\mathbf{G}\right) \mathbf{C}^{-1}\left(\phi^{-2} \mathbf{C}+\mathbf{G}\right), \\
& \mathbf{Q}_{3, \phi}=\phi^{-6} \mathbf{C}+3 \phi^{-4} \mathbf{G}+3 \phi^{-2} \mathbf{G} \mathbf{C}^{-1} \mathbf{G}+\mathbf{G C}^{-1} \mathbf{G} \mathbf{C}^{-1} \mathbf{G}, \\
& \mathbf{Q}_{3, \phi}=\phi^{-6} \mathbf{M}_{0}+3 \phi^{-4} \mathbf{M}_{1}+3 \phi^{-2} \mathbf{M}_{2}+\mathbf{M}_{2} \mathbf{M}_{0}^{-1} \mathbf{M}_{1} .
\end{aligned}
$$

Hence $\mathbf{Q}_{3, \phi}$ is sparse and can be calculated via a combination of $\mathbf{M}_{0}, \mathbf{M}_{1}$ and $\mathbf{M}_{2}$ which are themselves provided by inla.spde2.matern. Therefore we can use these matrices to construct $\mathbf{Q}_{3, \phi}$ for a solution to the SPDE approximation for $\alpha=3(\kappa=2)$. We simply need to take a new combination of these matrices which can be done within the TMB . cpp likelihood file. 


\section{Appendix B}

\section{Extended Kalman Filter}

The extended Kalman filter (EKF) output is obtained by updating the prediction equations,

$$
\begin{gathered}
\hat{\mathbf{Z}}_{k \mid k-1}=\mathbb{E}\left[\mathbf{Z}_{k} \mid \mathbf{Z}_{1:(k-1)}, \boldsymbol{\theta}\right]=h\left(\hat{\mathbf{X}}_{k \mid k-1}, \boldsymbol{\theta}\right), \\
\boldsymbol{R}_{k \mid k-1}=\boldsymbol{C} \boldsymbol{P}_{k \mid k-1} \boldsymbol{C}^{T}+\mathbf{E}_{k},
\end{gathered}
$$

where

$$
\boldsymbol{C}=\left.\frac{\partial h}{\partial \mathbf{X}(t)}\right|_{\hat{\mathbf{X}}_{k \mid k-1}}, \quad \text { and } \quad \mathbf{E}_{k}=\operatorname{Cov}\left(\boldsymbol{\epsilon}\left(t_{k}, \boldsymbol{\theta}\right)\right) .
$$

These equations are recursively updated using the Kalman gain equation,

$$
\boldsymbol{K}_{k}=\boldsymbol{P}_{k \mid k-1} \boldsymbol{C}^{T} \boldsymbol{R}_{k \mid k-1}^{-1},
$$

and the updating equations

$$
\begin{aligned}
\hat{\mathbf{X}}_{k \mid k} & =\hat{\mathbf{X}}_{k \mid k-1}+\boldsymbol{K}_{k} \boldsymbol{\psi}_{k}, \\
\boldsymbol{P}_{k \mid k} & =\boldsymbol{P}_{k \mid k-1}-\boldsymbol{K}_{k} \boldsymbol{R}_{k \mid k-1} \boldsymbol{K}_{k}^{T} .
\end{aligned}
$$

If we use the discretised version of the SDE such as (7.1) we can use the discrete-discrete EKF, where the state prediction equations are

$$
\begin{aligned}
& \hat{\mathbf{X}}_{k \mid k-1}=\hat{\mathbf{X}}_{k-1 \mid k-1}+\boldsymbol{\mu}\left(\hat{\mathbf{X}}_{k-1 \mid k-1}, \boldsymbol{\theta}\right)\left(t_{k}-t_{k-1}\right), \\
& \boldsymbol{P}_{k \mid k-1}=\boldsymbol{A P}_{k-1 \mid k-1} \boldsymbol{A}^{T}+\boldsymbol{\Sigma}(\boldsymbol{\theta}) \boldsymbol{\Sigma}(\boldsymbol{\theta})^{T},
\end{aligned}
$$

where

$$
\begin{aligned}
\boldsymbol{A} & =\left.\frac{\partial \boldsymbol{\mu}}{\partial \mathbf{X}(t)}\right|_{\hat{\mathbf{X}}_{k \mid k-1}}, \\
\boldsymbol{P}_{1 \mid 1} & =\operatorname{Var}\left(\mathbf{X}\left(t_{1}\right)\right), \\
\hat{\mathbf{X}}_{1 \mid 1} & =\mathbb{E}\left[\mathbf{X}\left(t_{1}\right)\right] .
\end{aligned}
$$

In the case that $h\left(\mathbf{X}\left(t_{k}\right), t_{k}, \boldsymbol{\theta}\right)=\mathbf{X}\left(t_{k}\right)$ we return to the standard linear Kalman filter framework. 


\section{Appendix $\mathrm{C}$}

\section{Template Model Builder Example Code}

Here we give a commented example of a .cpp file used to construct the negative joint log-likelihood function in TMB

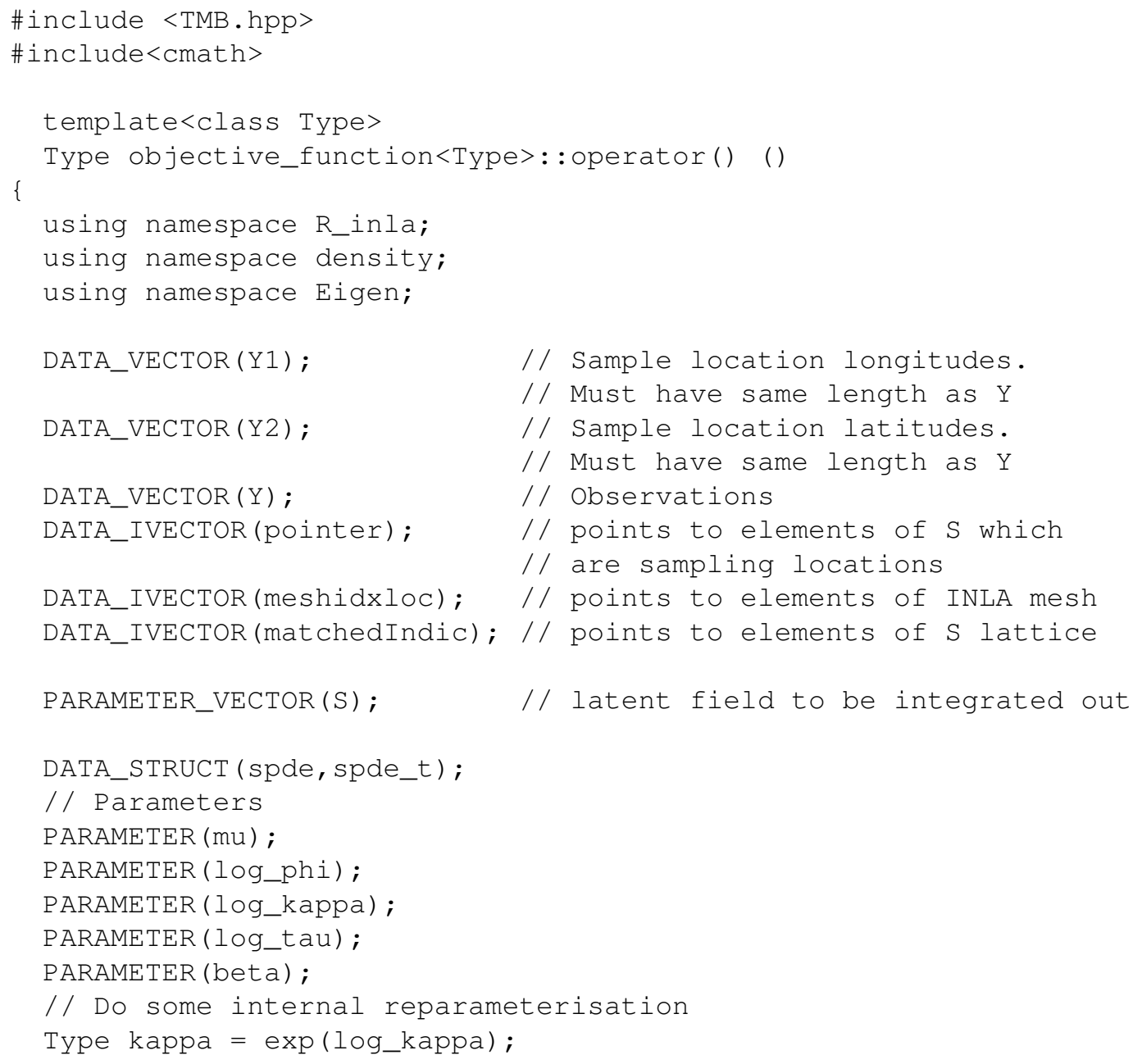




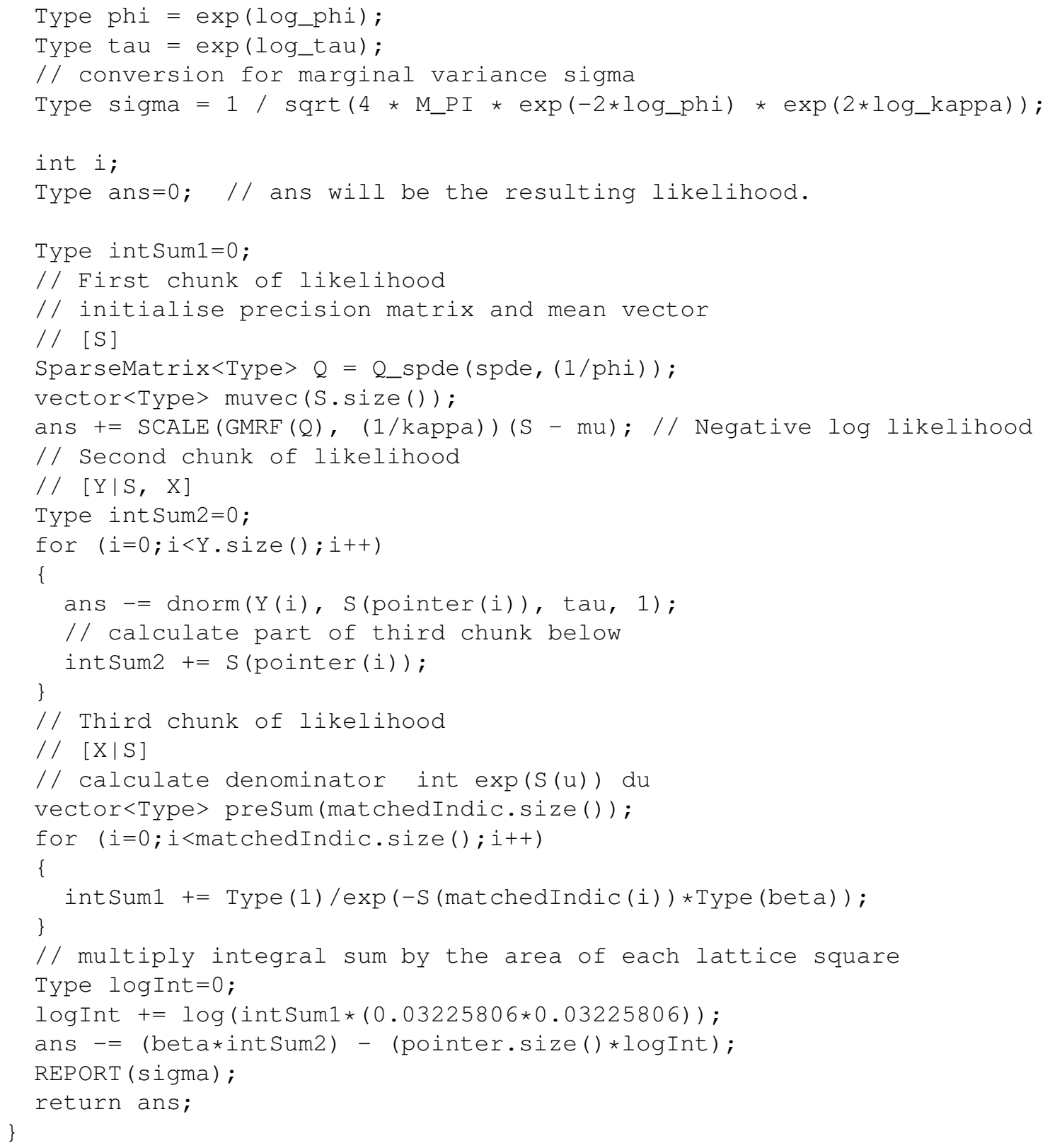




\section{Appendix D \\ Extra Plots}




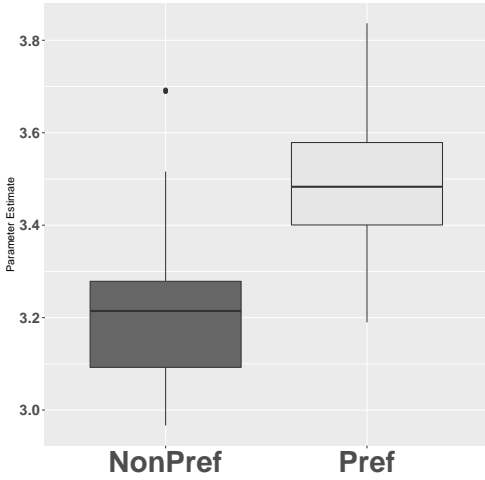

(a) Mean $(\mu)$

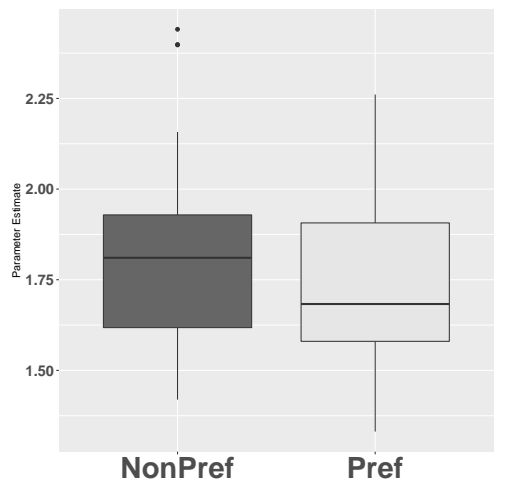

(c) Variance $\left(\sigma^{2}\right)$

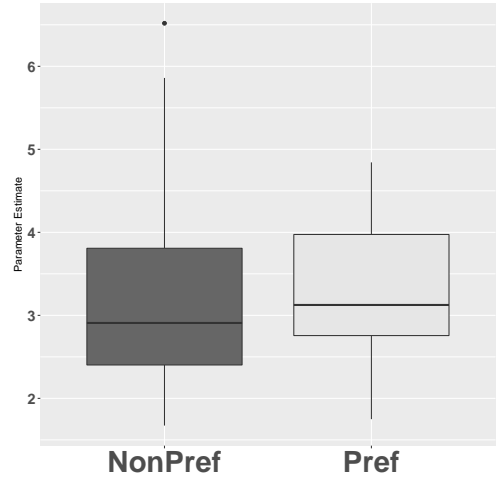

(b) Scale $(\phi)$

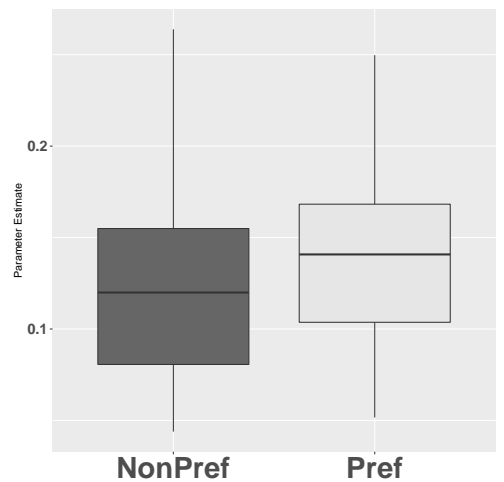

(d) Nugget variance $\left(\tau^{2}\right)$

Figure D.1: Comparison between preferential and standard MLEs for the field parameters over 50 data sets described in Section 9.1 consisting of a sub-sample of 9 tracks of 30 observations each. 


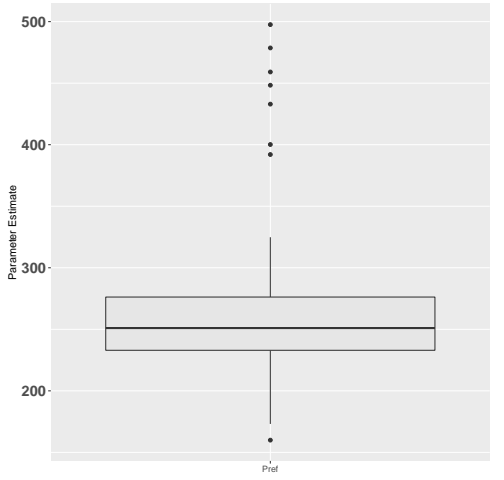

(a) $\alpha$

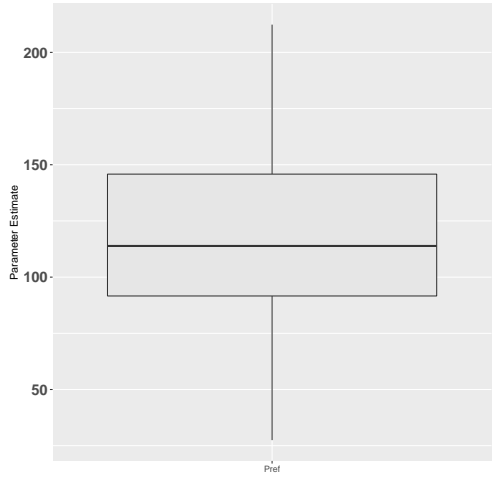

(b) $\beta$ Variance $\left(\operatorname{Diag}\left(\boldsymbol{\Sigma}_{\beta}\right)\right)$

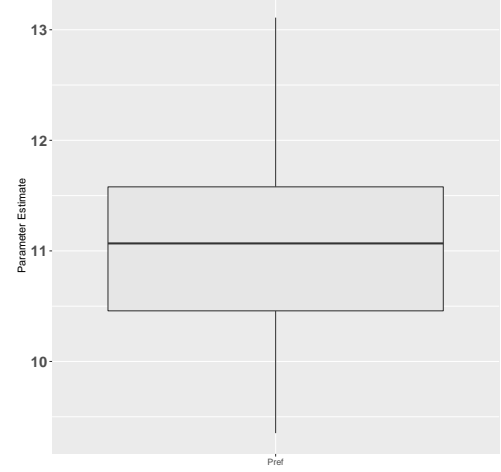

(c) Diffusion Variance $\left(\operatorname{Diag}\left(\boldsymbol{\Sigma}_{x}\right)\right)$

Figure D.2: Movement parameter estimates using the preferential model over 50 data sets described in Section 9.1 consisting of a sub-sample of 9 tracks of 30 observations each. 